\title{
Single and Multi-photon Laser Induced Fluorescence for Electric Thruster and Fusion Applications
}

Thomas E. Steinberger

West Virginia University, testeinberger@mail.wvu.edu

Follow this and additional works at: https://researchrepository.wvu.edu/etd

Part of the Plasma and Beam Physics Commons

\section{Recommended Citation}

Steinberger, Thomas E., "Single and Multi-photon Laser Induced Fluorescence for Electric Thruster and Fusion Applications" (2021). Graduate Theses, Dissertations, and Problem Reports. 8146.

https://researchrepository.wvu.edu/etd/8146

This Thesis is protected by copyright and/or related rights. It has been brought to you by the The Research Repository @ WVU with permission from the rights-holder(s). You are free to use this Thesis in any way that is permitted by the copyright and related rights legislation that applies to your use. For other uses you must obtain permission from the rights-holder(s) directly, unless additional rights are indicated by a Creative Commons license in the record and/ or on the work itself. This Thesis has been accepted for inclusion in WVU Graduate Theses, Dissertations, and Problem Reports collection by an authorized administrator of The Research Repository @ WVU. For more information, please contact researchrepository@mail.wvu.edu. 


\title{
Single And Multi-Photon Laser Induced Fluorescence For Electric Thruster and Fusion Applications
}

\author{
Thomas Edward Steinberger
}

\begin{abstract}
Dissertation submitted to the Eberly College of Arts and Sciences at West Virginia University

In partial fulfillment of the requirements for the degree of
\end{abstract}

Doctor of Philosophy

in

Physics

\author{
Earl E. Scime, Ph.D., Chair \\ Paul A. Cassak, Ph.D. \\ Timothy Good, Ph.D. \\ Tudor Stanescu, Ph.D.
}

Department of Physics and Astronomy

Morgantown, West Virginia

March 2021

Keywords: laser induced fluorescence, two-photon absorption laser induced fluorescence, three-photon laser induced fluorescence, Proto-MPEX, electric propulsion, fusion, iodine, deuterium

(C)2020 Thomas Edward Steinberger 


\section{Abstract}

Single and Multi-photon Laser Induced Fluorescence for Electric Thruster and Fusion Applications

Thomas Edward Steinberger

Laser-based diagnostics are increasingly sought after to investigate a variety of plasmas due to their non-perturbative capabilities. Specifically, laser induced fluorescence (LIF) provides a highly localized and precise spectroscopic technique to measure absolute density, temperature, and bulk flow. In this work, LIF and two-photon absorption laser induced fluorescence (TALIF) are used to investigate electric propulsion and fusion-relevant plasmas, respectively. Ion velocity distribution functions (IVDF) of singly ionized atomic iodine (I II) are measured for the first time and lineshape characteristics are presented for the diagnosis of Hall thrusters with iodine as a propellant. A non-perturbative technique of determining external magnetic field from Zeeman split iodine spectra is also discussed and results are shown.

Fusion-relevant plasmas are investigated in the Prototype Material Plasma Exposure eXperiment (Proto-MPEX) using TALIF. Ground state neutral deuterium velocity distribution functions are measured during Proto-MPEX operation. Absolute neutral density, neutral temperature, and neutral bulk flow are determined. Absolute neutral deuterium densities are calculated by calibrating measured deuterium velocity distributions with xenon and krypton. Calibrated density values from the two gases are compared. This work presents, for the first time, deuterium absolute densities calibrated using xenon, first proposed by Elliot et al. Temporal evolution of deuterium signal is shown over the entirety of a Proto-MPEX plasma pulse. Neutral density, temperature, and bulk flow dependence on radio frequency (rf) power and radial location is presented. Lastly, a novel three photon laser induced fluorescence (3pLIF) scheme is shown for krypton. 


\section{Acknowledgements}

The measurements presented in this work are truly a combined effort from an amazing network of people. I will try my best to acknowledge everyone that contributed and apologize to those who I forgot.

First, I would like to thank Dr. William Hargus at Edwards Air Force Base. Without his generosity, a significant portion of the work would not be possible. I would also like to thank the entire Proto-MPEX team for helping with TALIF measurements, even when it was a major inconvenience.

I would be remiss if I did not mention the Physics department machinists: Doug Mathess, J.R. Raber, and Carl Weber. The three of you have helped me in a pinch more times than I can count and have always provided me with high precision parts, no matter how ridiculous.

Next, I want to applaud my colleagues in Scime Lab. In no particular order: Regis John, your inquisitive nature always provided a unique aspect to my understanding and your attention to detail has caught me flat-footed more than once. Tyler Gilbert and Katey Stevenson, thank you for teaching me the operation and troubleshooting of the iodine diode laser. John McKee, you always were available for a quick chat and are a sounding board, while at the same time the perspective you offered reminded me to not be caught up in small details. Evan Aguirre, though our time in lab overlapped briefly, I found a lifelong friend. Thank you for your help with the early stages of the iodine plasma source. Andrew Jemiolo, you are the epitome of chill and a constant reminder to put my best foot forward, but also to keep personal happiness separate from work. You are a true friend and a great colleague. Derek Thompson, thank you for being a role model as a scientist. I appreciate you humoring my stupid questions and offering advice to keep me on track. Mitchell Paul, I have to thank you for your support in the lab and picking up the slack when I couldn't. Without you, I wouldn't be writing this right now. David Caron, thank you for being an exemplary office mate and an even better friend. You have put up with my misunderstandings of simple topics and, even worse, endless hours of monotonous music. Thank you. Cuyler Beatty, you have repeatedly proved to be one of the best friends I will have in my lifetime. You are selfless and truly willing to help out whenever you can. No matter what obstacles were thrown your way, you always remained the beacon of positivity. I thank you for being such a good friend and going out of your way to accommodate Kayla and me more times than I can count. I wish you the best of luck with your future endeavors in California (or wherever the road takes you).

Jacob McLaughlin, I don't think a simple thanks amounts to the gratitude you deserve. The countless trips you took with me to ORNL were invaluable. I think it is safe to say that without your help and dedication, I would not be where I am today. I hope that all the trips and 
obstacles involved with TALIF and the Cobra Stretch weren't too traumatic! To that end, if you ever need help with anything, just let me know-I owe you one.

Dr. Theodore Biewer, you were a critical component to this work, and I thank you for the amount of time you sacrificed to help me out with this project, both with the science and for relaying all the ORNL notifications I missed. I couldn't ask for a better advisor "away from home". Thank you for marching down the TALIF rabbit hole with me, and I hope that I get the opportunity to work with you again!

Ted Lane, Kaustubh Rajwade, and Matt Flaugh: I don't think I can ask for better colleagues and lifelong friends. You were my first friends when I arrived to WVU, and I attribute my early success to being around you all.

Pedram Tavadze. I don't think the first few years of grad school would have been the same without you. I have to say thanks for everything. I always knew that I could count on you for anything from studying for quals to being in my wedding. Even though our schedules are starting to get busier, I hope we can always find time to grab a beer and catch up.

Earl Scime, I owe you a lot of gratitude. First, thank you for giving me the opportunity to work in your lab and learn so much. Thanks for putting up with me stubborn attitude. I hope that I didn't cause you too much stress. I am lucky to have an advisor that took so much time to work with me and make sure I was successful. Thank you.

Lea Steinberger, thank you for all the support you have shown me over my lifetime. I hope that I can help impact your life the way you have mine.

To my parents: thank you for always being there for me and encouraging me. I cannot begin to think of the things you sacrificed so that I can be where I am today. I am truly grateful. I hope that someday I can be as good as parents to my children as you were to me. I love you guys.

Finally, a tremendous thank you to my beautiful wife, Kayla Steinberger. You have always stuck by me and supported me through this entire grad school experience. You always took time to help me and listen to me, no matter what it was about. You sat through my endless presentations, monotonous physics talks, stupid rants, and put up with all of my off-the-wall quirks (like keeping the volume in multiples of 5). You will always be my greatest adventure, and I cannot thank you enough for everything you have done for me over the last decade! I love you! 


\section{Contents}

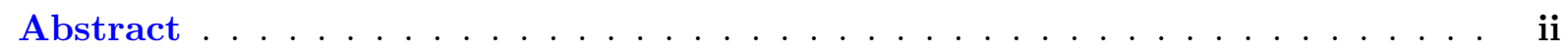

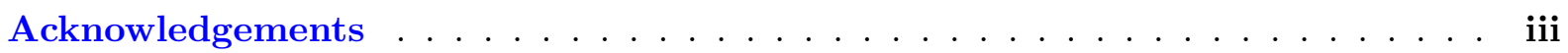

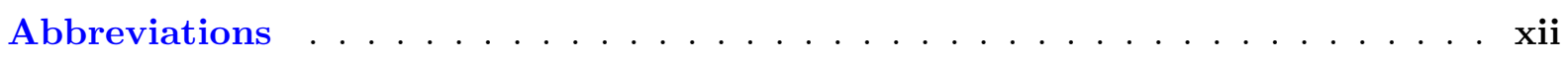

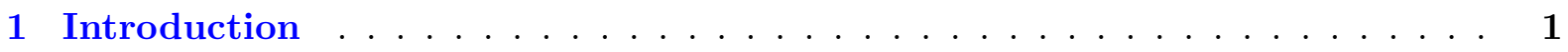

1.1 Thruster Motivation and Context . . . . . . . . . . . . . 2

1.2 Hall Thrusters . . . . . . . . . . . . . . . . . . 4

1.3 Plasma Material Interaction and Fusion-relevant Plasmas . . . . . . . . . . 8

1.3.1 Charge Exchange . . . . . . . . . . . . . . . . . . 9

1.3.2 Role of Neutrals in the L-to-H Mode Transition . . . . . . . . . . . . . . . 10

1.3.3 Neutral Recycling and Retention . . . . . . . . . . . . . . . . 12

1.4 Neutral Particle Diagnostic Techniques . . . . . . . . . . . . . . . . . . 12

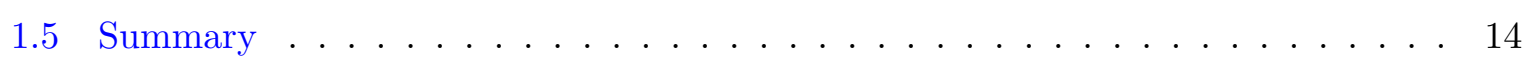

2 Single and Multi-photon Laser Induced Fluorescence . . . . . . . . . 15

2.1 Introduction . . . . . . . . . . . . . . . . . . . 15

2.2 Laser Induced Fluorescence . . . . . . . . . . . . . . . . . . 16

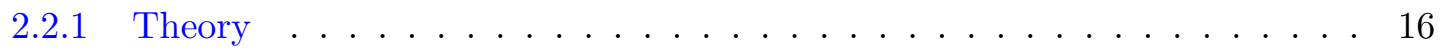

2.2 .2 LIF Transition Specifics . . . . . . . . . . . . . . . . . . . . . 19

2.3 Two-photon Absorption Laser Induced Fluorescence . . . . . . . . . . . . . 20

2.3 .1 Theory . . . . . . . . . . . . . . . . . 20

2.3.2 TALIF Transition Specifics . . . . . . . . . . . . . . . 22

2.3.3 Absolute Density Calibration . . . . . . . . . . . . . . . . 24

2.4 Three-photon Absorption Laser Induced Fluorescence . . . . . . . . . . . . 26

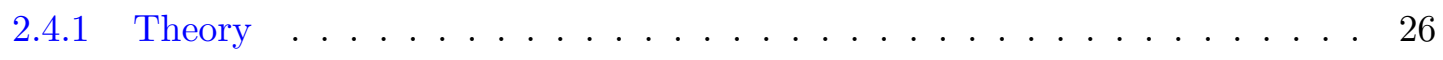

2.4 .23 pLIF Transition Specifics . . . . . . . . . . . . . . . 28

3 Broadening Mechanisms . . . . . . . . . . . . . . 30

3.1 Natural Linewidth . . . . . . . . . . . . . . . . . . . . 30 
3.2 Pressure/Stark Broadening . . . . . . . . . . . . . . . . . . . . . 31

3.3 Laser Linewidth . . . . . . . . . . . . . . . . . . . . . . . 31

3.4 Zeeman Splitting . . . . . . . . . . . . . . . . . . . . . 35

3.5 Hyperfine Splitting . . . . . . . . . . . . . . . . . . . . . . . . 42

3.6 Isotopic Splitting . . . . . . . . . . . . . . . . . . . . . . . 43

3.7 Doppler Broadening ........................... . . 46

3.8 Saturation Broadening . . . . . . . . . . . . . . . . . . . . . . . 48

3.9 Summary ............................ 50

4 Experimental Configuration .........................51

4.1 Iodine Laser Induced Fluorescence . . . . . . . . . . . . . . . . . . . . . . . . 51

4.1 Iodine Laser System . . . . . . . . . . . . . . . . . 51

4.1 .2 Iodine Laser Specifications . . . . . . . . . . . . . . . . . 53

4.1.3 Iodine Data Acquisition (DAQ) System and Layout . . . . . . . . . . 55

4.1.4 Iodine Water-cooled Reference Cell . . . . . . . . . . . . . . . 58

4.2 Deuterium Two-photon Absorption Laser Induced Fluorescence . . . . . . . . . . 60

4.2 .1 TALIF Laser System . . . . . . . . . . . . . . . . . . . . 61

4.2.2 TALIF Laser Specifications . . . . . . . . . . . . . . . . 63

4.2.3 Ultra Violet Beamline ....................... 68

4.2 .4 Confocal Optics ........................ 70

4.2.5 TALIF Electronics and Experimental Layout . . . . . . . . . . . . 72

4.2.6 Prototype Material-Plasma Exposure eXperiment (Proto-MPEX) . . . . . 78

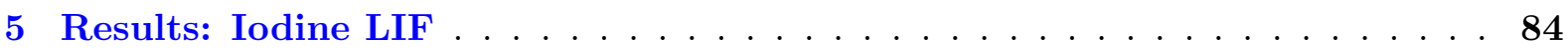

5.1 I II Passive Spectroscopy _. . . . . . . . . . . . . . . . . . . . . . . 84

5.2 I II LIF . . . . . . . . . . . . . . . . . . . . . . . . . . . . . . 86

5.3 Zeeman-split I II LIF . . . . . . . . . . . . . . . . . . . . . . . . . . 94

5.4 Summary ............................. . 96

6 Results: Deuterium TALIF ......................... 99

6.1 Xenon Calibration in Proto-MPEX . . . . . . . . . . . . . . 99

6.2 Proto-MPEX Operation for Preliminary TALIF Measurements . . . . . . . . . 102

6.3 RF Power Variation Measurements . . . . . . . . . . . . . . . . . . . . . . 104

6.4 Radial Measurements . . . . . . . . . . . . . . . . . . 109

6.5 Summary .............................. 112

7 Discussion and Future Work . . . . . . . . . . . . . . . . . . . . . . . 114

7.1 I II LIF and Applications to Ion Thrusters . . . . . . . . . . . . . . . . . . . . . 114

7.2 Deuterium TALIF and Applications to Fusion-relevant Plasmas . . . . . . . . . 116

7.2.1 SOLPS Simulations of Proto-MPEX . . . . . . . . . . . . 116 
7.2.2 TALIF Discussion and Future Work . . . . . . . . . . . . . . 117

A Convolution of Gaussian and Lorentzian Lineshapes . . . . . . . . . . 121

A.1 Convolution of Identical Gaussian Functions . . . . . . . . . . . . . . . . . . . 121

A.1.1 Relations . . . . . . . . . . . . . . . . . . . . . . . . 121

A.1.2 Derivation . . . . . . . . . . . . . . . . . . . . 122

A.2 Convolution of Identical Lorentzian Functions . . . . . . . . . . . . . . . . 125

A.2.1 Derivation . . . . . . . . . . . . . . . . . . . . 125

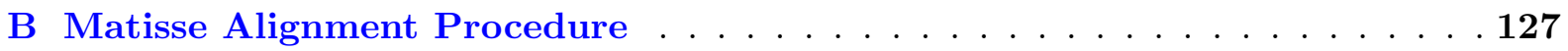

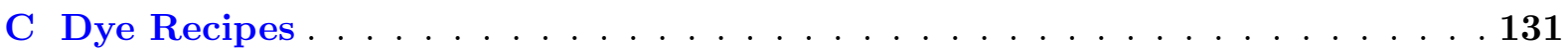

C.1 Matisse Pyridine 1 (LDS698) Recipe (I II LIF) . . . . . . . . . . . . . . . 131

C.2 Sirah Cobra Stretch Rhodamine Dye Recipe (D I, Xe I, Kr I TALIF) . . . . . . . 132

D Energy Conversion Curves for TALIF Measurements . . . . . . . . . 133

D.1 Deuterium . . . . . . . . . . . . . . . . . . . . . . . 133

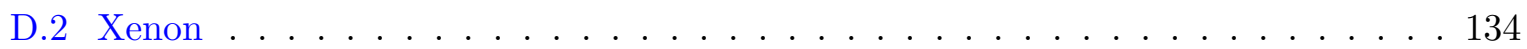

D.3 Krypton . . . . . . . . . . . . . . . . . . . . . . . 135

E Hamamatsu Data . . . . . . . . . . . . . . . . . . . 136

E.1 Gain . . . . . . . . . . . . . . . . . . . . 136

E.2 Quantum Efficiency . . . . . . . . . . . . . . . . . . 137

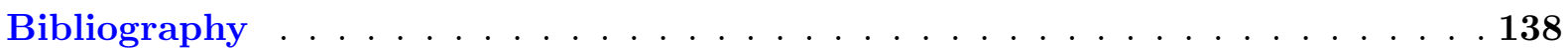




\section{List of Figures}

1.1 Parameter Space of Plasma Physics . . . . . . . . . . . . . . . . . 2

1.2 Hall Thruster Schematic . . . . . . . . . . . . . . . . . . . . . . . . 4

1.3 Hall Effect Cartoon . . . . . . . . . . . . . . . . . . . . . . . 5

1.4 Example Divertor . . . . . . . . . . . . . . . . . . . 9

$1.5 \mathrm{D}_{\alpha}$ Emission in Divertor Region . . . . . . . . . . . . . . . . . . . . 11

1.6 Pedestal Pressure vs. Pedestal Density for Super H-mode Transitions . . . . . . 12

2.1 I II Partial Grotrian Diagram . . . . . . . . . . . . . . . . . . . . . . 20

2.2 TALIF Partial Grotrian Diagrams . . . . . . . . . . . . . . . . 23

2.3 TALIF Filter Transmissions . . . . . . . . . . . . . . . . . . . . 25

2.43 pLIF Krypton Partial Grotrian Diagram . . . . . . . . . . . . . . . 28

3.1 Laser Linewidth Compared to Deuterium NVDF . . . . . . . . . . . . . . . . . 33

3.2 Laser Linewidth Compared to Xenon NVDF . . . . . . . . . . . . . . . . 34

3.3 Relative Linewidth Error . . . . . . . . . . . . . . . . . . . . 35

3.4 Zeeman Transitions in Weak-Field Limit . . . . . . . . . . . . . . . . . . . . . 40

3.5 Zeeman Transitions in Strong-Field Limit . . . . . . . . . . . . . . . . . 40

3.6 Zeeman Effect on Measured Temperature for Proto-MPEX Conditions . . . . . . 41

3.7 I II Hyperfine Levels . . . . . . . . . . . . . . . . . . . . . . . . . 44

3.8 Simulated Doppler Distributions for Different Thermal Temperatures . . . . . . . 49

4.1 Matisse Optical Configuration . . . . . . . . . . . . . . . . . . . . . 52

4.2 Birefringent Filter Wavelength Orders . . . . . . . . . . . . . . . . . 54

4.3 Matisse Dye Curves . . . . . . . . . . . . . . . . 55

4.4 Iodine LIF Experimental Configuration . . . . . . . . . . . . . . . . 55

4.5 Spectral Shift vs. Lock-in Sample Rate . . . . . . . . . . . . . . . . . . . 57

4.6 Iodine Plasma Cell . . . . . . . . . . . . . . . . . . 58

4.7 Iodine Plasma Source with Magnet Configuration . . . . . . . . . . . . . . . 60

4.8 TALIF Dye Laser Path . . . . . . . . . . . . . . . . . . . . . . . . . . . 61

4.9 TALIF Laser System Beam Diameter . . . . . . . . . . . . . . . . . . . 63

4.10 Laser Dye Curve . . . . . . . . . . . . . . . . . . . . . . . . . 64

4.11 Lorentzian-Gaussian Lineshape Comparison . . . . . . . . . . . . . . . . 67

4.12 Ultra-violet Beamline . . . . . . . . . . . . . . . . . . . . . . . . 68 
4.13 Mirror-Camera-Flipper Setup . . . . . . . . . . . . . . . . . . 70

4.14 Confocal Collection Design . . . . . . . . . . . . . . . . 71

4.15 TALIF Electronics Box Diagram for Deuterium Measurements . . . . . . . . . 72

4.16 BoxCar Background Subtraction Schematic . . . . . . . . . . . . . . . 74

4.17 Energy-per-Pulse Rise Times . . . . . . . . . . . . . . . . . . . . 75

4.18 TALIF Electronics Box Diagram for Calibration Measurements . . . . . . . . 76

4.19 Detector Gain . . . . . . . . . . . . . . . . . . . . . . 77

4.20 Detector Quantum Efficiency . . . . . . . . . . . . . . . . . 78

4.21 Proto-MPEX Vacuum Vessel . . . . . . . . . . . . . . . . . . . . . . . 79

4.22 Magnetic Field Profile . . . . . . . . . . . . . . . . . . . . 80

5.1 I II Transition Intensity vs. Pressure . . . . . . . . . . . . . . . 85

5.2 Pump and Fluorescence Spectra . . . . . . . . . . . . . . 85

5.3 Example I II IVDF . . . . . . . . . . . . . . . . . . . . . . . . . 86

5.4 I II LIF Pressure Variation . . . . . . . . . . . . . . . . . 87

5.5 Possible Hyperfine Structure of I II . . . . . . . . . . . . . . . . . . . . 89

5.6 Lamb-dip Experimental Configuration . . . . . . . . . . . . . . . . . . . 90

5.7 IVDF Measured with Saturated Spectroscopic Technique . . . . . . . . . . . . . . 91

5.8 I II Hyperfine Structure for Different $T_{\text {ion }} \ldots \ldots \ldots \ldots$. . . . . . . . . . 91

5.9 Doppler-free Saturation Spectroscopy Experimental Configuration . . . . . . . . 92

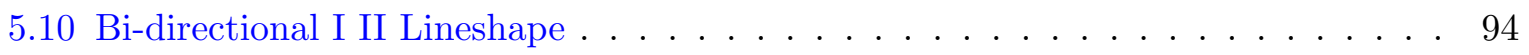

5.11 I II Zeeman Splitting . . . . . . . . . . . . . . . . . . . . . . . 95

5.12 I II $\pi$ and $\sigma$ Peaks . . . . . . . . . . . . . . . . . . . . . . . 96

$5.13 \sigma$-peak Separation for Range of External Magnetic Fields . . . . . . . . . . . . . 97

5.14 I II Zeeman-splitting Constant . . . . . . . . . . . . . . . . . . . 98

6.1 Typical Xe Calibration Measurements in Proto-MPEX . . . . . . . . . . . . 100

6.2 Typical Kr Calibration Measurements in Proto-MPEX . . . . . . . . . . . . . . . 101

6.3 Typical Pressure Evolution in Proto-MPEX . . . . . . . . . . . . . . . . 102

6.4 Typical Deuterium NVDF Over Entire Plasma Pulse . . . . . . . . . . . . . . 103

6.5 Example D TALIF Time Series Plot . . . . . . . . . . . . . . . . . . 104

6.6 Temporal rf Variation of Deuterium NVDFs . . . . . . . . . . . . . . 105

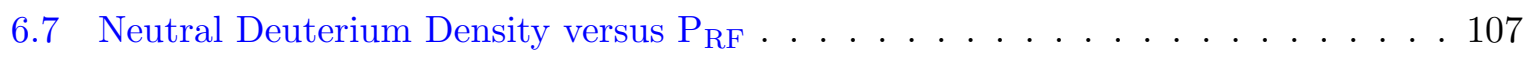

6.8 Neutral Deuterium Temperature versus $\mathrm{P}_{\mathrm{RF}} \ldots \ldots \ldots$. . . . . . . . . . . 108

6.9 Neutral Deuterium Bulk Flow versus $P_{R F} \ldots \ldots$. . . . . . . . . . . . . . 109

6.10 Temporal Radial Variation of Deuterium NVDFs . . . . . . . . . . . . . 110

6.11 Neutral Deuterium Density versus $r \ldots \ldots \ldots \ldots 111$

6.12 Neutral Deuterium Temperature versus $r \ldots \ldots$. . . . . . . . . . 111

6.13 Neutral Deuterium Bulk Flow versus $r \ldots \ldots$. . . . . . . . . . . 112 
7.1 I II VDF Measured with Portable Diode Laser . . . . . . . . . . . . . . . . . . . 115

7.2 SOLPS Neutral Deuterium Density Profiles . . . . . . . . . . . . . 117

7.3 SOLPS Neutral Molecular Deuterium Temperature Profiles . . . . . . . . . 118

B.1 Vertical and Horizontal Adjustments for M1 . . . . . . . . . . . . . . . 129

B.2 Vertical and Horizontal Adjustments for M4 . . . . . . . . . . . . . . . . 129

B.3 Vertical and Horizontal Adjustments for Thick Etalon . . . . . . . . . . . . . 130

D.1 Deuterium Conversion Curve for meausurements on 18 July . . . . . . . . . . 133

D.2 Deuterium Conversion Curve for meausurements on 22 July . . . . . . . . . . . 134

D.3 Xenon Conversion Curve for meausurements on 18 July . . . . . . . . . . . 134

D.4 Xenon Conversion Curve for meausurements on 22 July . . . . . . . . . . . . 135

D.5 Krypton Conversion Curve for meausurements on 22 July . . . . . . . . . . . 135 


\section{List of Tables}

1.1 Hall Thruster Propellant Characteristics . . . . . . . . . . . . . . . 6

2.1 TALIF Schemes . . . . . . . . . . . . . . . . . . . . . 23

2.23 3LIF Krypton Schemes . . . . . . . . . . . . . . . . . . . 29

3.1 Weak and Strong Magnetic Field Thresholds . . . . . . . . . . . . . . . 39

3.2 Hyperfine Relative Intensity Rules for I II . . . . . . . . . . . . . . . . . . . 44

4.1 TALIF Laser System Characteristics . . . . . . . . . . . . . . . . . . . 67

4.2 Proto-MPEX Suite of Diagnostics . . . . . . . . . . . . . . . . 83

5.1 I II Hyperfine Fitting Results . . . . . . . . . . . . . . . . . . . . . . . . . . . 89 


\section{Abbreviations}

\begin{tabular}{|c|c|}
\hline $3 \mathrm{pLIF}$ & Three Photon Laser Induced Fluorescence \\
\hline AXUV & Absolute Extended Ultra Violet \\
\hline $\mathrm{BBO}$ & Beta Barium Borate \\
\hline BIFI & Birefringent Filter \\
\hline $\mathrm{CaF}_{2}$ & Calcium Fluoride \\
\hline $\mathrm{CW}$ & Continuous Wave \\
\hline DAQ & Data Acquisition \\
\hline EG & Ethylene Glycol \\
\hline EMI & Electromagnetic Interference \\
\hline $\mathrm{EPH}$ & Glycol Monophenyl Ether \\
\hline FWHM & Full-width at Half-maximum \\
\hline $\mathrm{HT}$ & Hall Thruster \\
\hline HWHM & Half-width at Half-maximum \\
\hline I II & Singly Ionized Atomic Iodine \\
\hline IVDF & Ion Velocity Distribution Function \\
\hline ITER & The International Thermonuclear Experimental Reactor \\
\hline LDS698 & Pyridine 1 \\
\hline LIF & Laser Induced Fluorescence \\
\hline MPEX & Material Plasma Exposure eXperiment \\
\hline Nd:YAG & Neodymium-doped Yttrium Aluminum Garnet \\
\hline NVDF & Neutral Velocity Distribution Function \\
\hline $\mathrm{OC}$ & Output Coupler \\
\hline OD & Optical Depth \\
\hline OES & Optical Emission Spectroscopy \\
\hline ORNL & Oak Ridge National Laboratory \\
\hline $\mathrm{PFC}$ & Plasma Facing Components \\
\hline PMI & Plasma Material Interaction \\
\hline Proto-MPEX & Prototype Material Plasma Exposure eXperiment \\
\hline $\mathrm{rf}$ & Radio Frequency \\
\hline RMSE & Root Mean Squared Error \\
\hline
\end{tabular}




$\begin{array}{ll}\text { SH } & \text { Super H-mode } \\ \text { SOL } & \text { Scrape-off Layer } \\ \text { SXR } & \text { Soft X-Ray } \\ \text { TALIF } & \text { Two-photon Absorption Laser Induced Fluorescence } \\ \text { TGG } & \text { Terbium Gallium Garnet } \\ \text { UV } & \text { Ultra Violet } \\ \text { VDF } & \text { Velocity Distribution Function }\end{array}$


Dedicated to my best friend, my partner in crime, my rock, and love of my life-Kayla Steinberger. Thanks for putting up with my shenanigans. 


\section{Chapter 1}

\section{Introduction}

It is estimated that a large fraction of the matter in the universe exists in a plasma state (that is, an electrically charged gas that is composed of positively charged ions and negatively charged electrons or ions that exhibits collective behavior and is quasi-neutral). As a result, plasma processes are responsible for several physical world phenomena. It is common to parameterize plasmas in terms of the (often ion) thermal temperature $T_{i}$ and the (often electron) density $n_{e}$. Figure 1.1 is a visual representation of the plasma parameter space with several plasmas displayed. A large range of temperatures and densities describe the parameter space of plasma physics; ion temperatures span values from room temperature $(0.026 \mathrm{eV})$ to $10^{6} \mathrm{eV}$ and densities vary from $10^{6}$ to $10^{34} \mathrm{~m}^{-3}$ [1]. While several experiments are shown, the list is not exhaustive. This work focuses on two specific experimental regimes shown: (1) Hall thrusters (HT) and (2) plasma-material interaction (PMI) studies (Proto-MPEX region in Fig. 1.1). Understanding how the plasma changes from one spatial location to another and how particles interact with the device walls in each of these systems is critical to the operation of both HTs and Proto-MPEX/fusion devices. Common diagnostics that measure particle densities and temperatures perturb the plasma (Langmuir probes for example) and tend to provide measurements in one dimension only. Plasma-surface interactions are inherently three-dimensional [2] and non-perturbative diagnostics that are spatially resolved are essential to correctly measure plasma dynamics in these systems. The plasma dynamics for the two systems investigated here are explored using similar laser-based methods, capable of spatially resolving temperatures, 


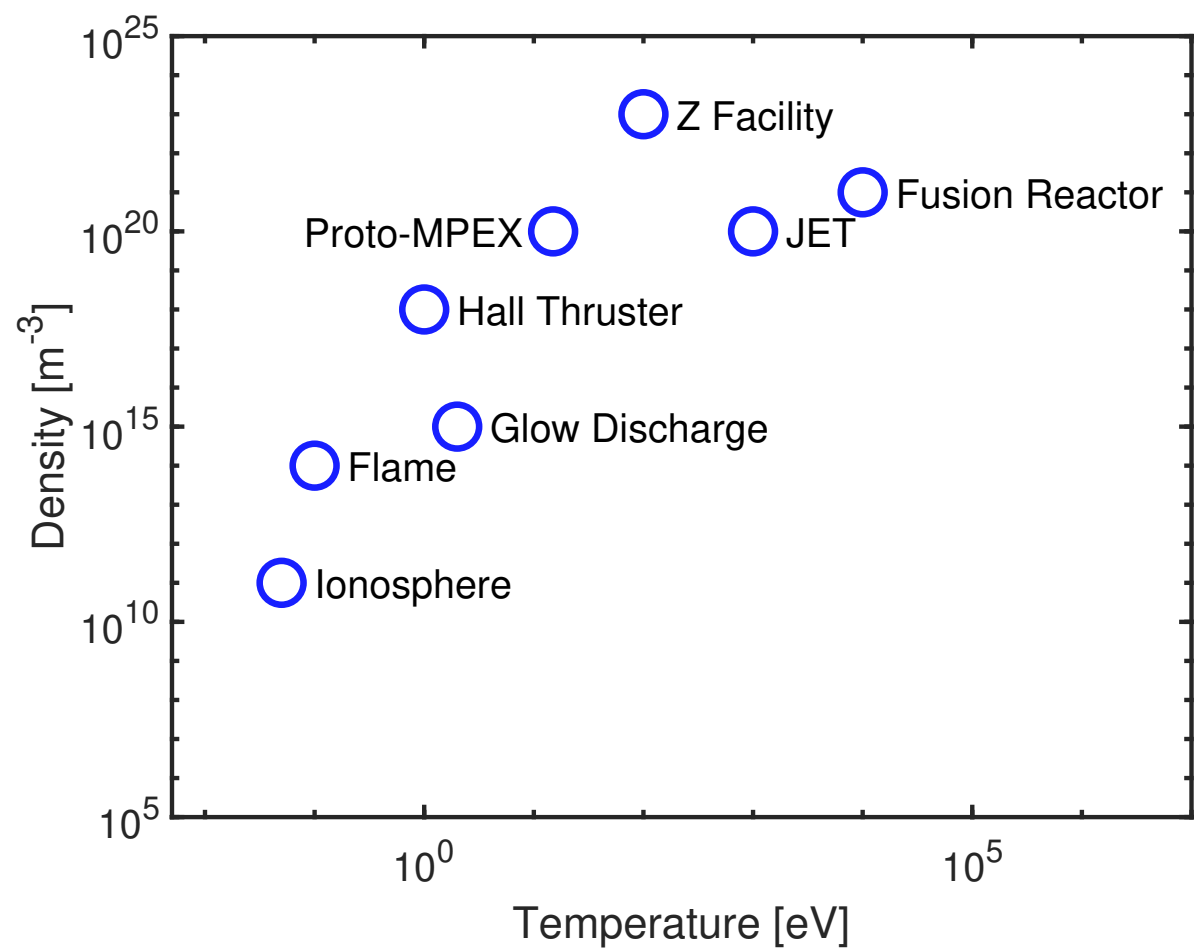

Figure 1.1: Parameter space of plasmas. A select set of plasmas are shown by the blue circles.

bulk flows, and densities. Velocity distribution functions (VDFs) are easily accessible in both systems using laser induced fluorescence (LIF) and reveal important plasma characteristics for each system. Species bulk flow, temperature, and density are reported for electric thrusters and fusion-relevant plasmas. Novel iodine ion LIF measurements are presented for future diagnosis of HTs using iodine as a propellant. Significant atomic structure for iodine ions is observed from measured spectral lineshapes. Neutral densities are measured at different radial locations and different radio frequency (rf) powers in Proto-MPEX and, for the first time, have been absolutely calibrated with xenon.

\subsection{Thruster Motivation and Context}

Thrusters and space travel have been a highly investigated field of study since Tsiolkovsky, Oberth, and Goddard first laid the theoretical and experimental groundwork for rockets and space travel in the early 1900's [3]. American physicist Goddard made significant contributions to rocket science through his earliest designs which used a combustible solid fuel that improved on previous designs [4]. In 1926, Goddard successfully launched a rocket with liquid fuel (liquid oxygen and gasoline) and since then, a range of fuel candidates and thruster designs have been 
studied to provide the most efficient and controlled operation for rocket propulsion. Space travel systems remain expensive and only economically developed countries have had the finances required to fund large space exploration experiments [3]. Therefore, more economical options for space propulsion are a necessity for wider access to space-a problem that is, in part, solved by electric propulsion. Electric propulsion was first proposed over 100 years ago by Goddard. He suggested the idea to create a device that used electrical potentials to accelerate electrons [5]. Electric propulsion has since become integral to the design of many thrusters. In the literature, thruster performance is typically described by two metrics: thrust and specific impulse $\left(I_{\mathrm{sp}}\right.$ or average bulk propellant exhaust velocity $v_{\mathrm{ex}}[3]$ ). Thrust is calculated from the exhaust velocity and time rate of change in mass:

$$
T=v_{\mathrm{ex}} \cdot \dot{m}
$$

where $\dot{m}$ is the mass flow rate. Increasing the exhaust velocity increases the thrust for the same change in ejected propellant mass. Therefore, by carefully controlling the exhaust velocity, the fuel efficiency of a thruster is improved. The specific impulse is defined as the change in momentum of the spacecraft per change in ejected mass:

$$
I_{\mathrm{sp}}=\frac{d p}{d m}=\frac{T}{\dot{m}}=v_{\mathrm{ex}}
$$

This quantity is equivalent to the average exhaust velocity [3]. Commonly, the specific impulse is measured using thrust stands in which a sustained physical displacement of the thruster from its original location is related to the thrust. This method of measuring specific impulse and thrust only provides the global value and reveals no spatially resolved information about exhaust motion. In this work, spatially resolved measurements of the exhaust velocity is the focus for development of a new HT diagnostic technique.

There are several types of thrusters that have been deployed in space. The most common thrusters are typically categorized into three types: (1) electrothermal thrusters, (2) electrostatic thrusters, and (3) electromagnetic thrusters. In depth discussions of each of these types of thruster is outside the scope of this work and the reader is directed to Refs. [3] and [5] for details of each category of thruster. For this work, Hall thrusters are the primary focus. 


\section{$1.2 \quad$ Hall Thrusters}

Hall thrusters are a type of electrostatic thruster used for electric propulsion systems and were among the first thrusters implemented on spacecraft and satellites. Hall thrusters became a standard thruster in the late 2000s (see Fig. 2 in Ref. [3]). The general operation of a HT is to create a plasma and accelerate the heavy ions out of the thruster using a two-stage electromagnetic process. Figure 1.2 shows the electromagnetic field geometry of a cylindrical HT. An axial electric field is crossed with a radial magnetic field to create an azimuthal $\vec{E} \times \vec{B}$ drift, trapping magnetized electrons. The trapped electron current, bound to follow the $\vec{E} \times \vec{B}$

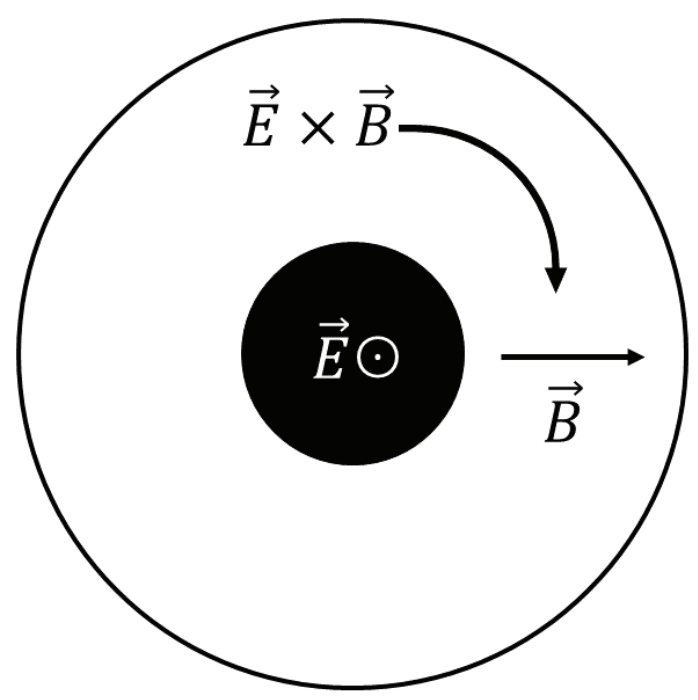

Figure 1.2: Simple field schematic for a common design of Hall thrusters. An axial electric field is crossed with a radial magnetic field to create an azimuthal $\vec{E} \times \vec{B}$ drift that traps the strongly magnetized electrons and allows for the axial acceleration of the largely unmagnetized ions.

drift, is called the Hall current. The electrons are trapped in the HT channel since their gyroradius is much smaller than the ions. However, the ion motion is dominated by the electric field since the ions are much heavier than electrons and their gyroradius is large.

Hall thrusters are sometimes referred to as "Hall effect thrusters," but this is a misnomer since the operation of a HT is critically dependent on suppressing the Hall effect. The Hall effect must be mitigated to ensure good confinement of electrons. Figure 1.3 shows a simple cartoon that describes the Hall effect. The applied magnetic field exerts a force on the Hall current towards the anode. If the electrons reach the anode, an electric field $\vec{E}_{\text {ind }}$ directed towards the anode (opposite of the applied electric field) is induced. A second electron drift $\left(\vec{E}_{\text {ind }} \times \vec{B}\right)$, opposing 
the first, destroys the electron confinement. In this case, it is assumed that the electrons are carrying all the current. To minimize the Hall effect in HTs, the electron flux from the $\vec{E} \times \vec{B}$

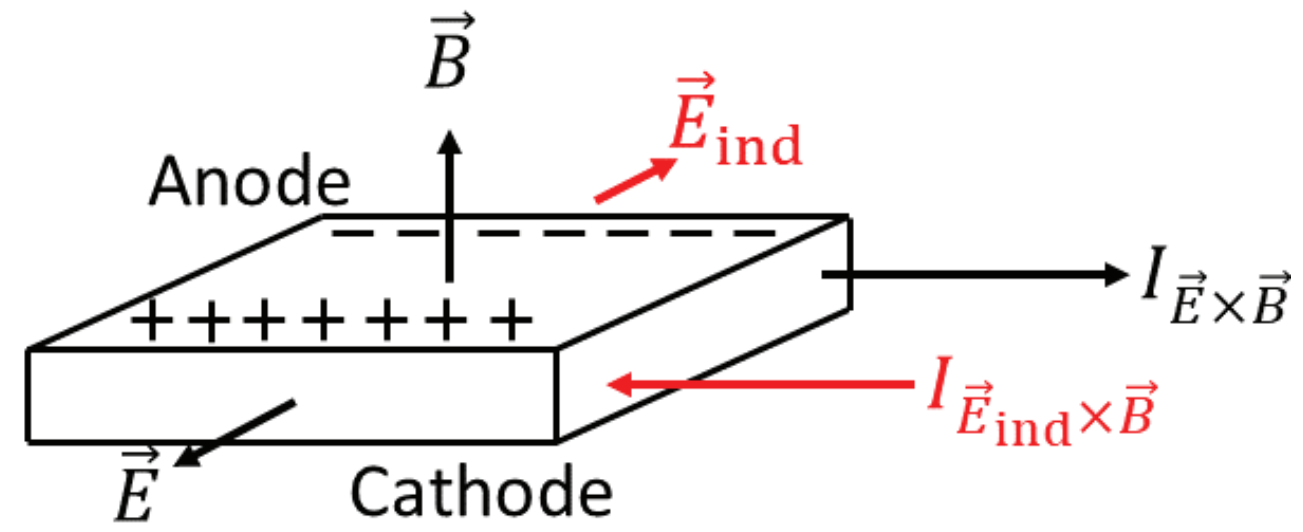

FiguRE 1.3: Simplified cartoon illustrating the Hall effect in a simple conducting slab.

must be larger than the cross field electron drift. The ratio of these two fluxes is known as the Hall parameter and is equivalent to the ratio of the electron cyclotron frequency to the electron collision frequency. The Hall parameter is sufficiently large for cylindrical HTs. However, even the best designs still observe some loss of particles to the wall of the thruster-thus, low secondary electron coefficient material should also be used for the walls. Magnetized plasmamaterial interactions, in plasma conditions similar to HTs, have been extensively studied at WVU by Thompson [6]. He found that the interaction of a magnetized plasma with a nonmagnetic surface is a three-dimensional structure and magnetic influences are more significant than predicted. While Thompson investigated non-magnetic surface interactions with a magnetized plasma, the particle flux to HT walls and the anode have been limited by magnetically insulating the HT anode [7].

Several investigations of physical processes, instabilities, and designs have been conducted and are important to the development of HTs. However, the results presented here focus on diagnostic methods for iodine, an alternative propellant for HTs, and those other topics are outside the scope of this work. The motivated reader is directed to Ref. [8] and references therein for discussion of simulation results and physical processes occurring in a HT and are referred to Ref. [9] for discussion of variants in designs of HTs.

The most common aims for HT design are to maximize thrust while optimizing the efficiency of the propellant and preserving the integrity of the thruster. While increasing thrust depends 
on several factors (ionization order, for example [10]), the most commonly investigated factor is propellant choice. Comparisons of common HT propellants are shown in Tab. 1.1.

\begin{tabular}{ccccc}
\hline \hline & $\begin{array}{c}\text { First Ionization } \\
\text { Potential } \\
(-)\end{array}$ & $\begin{array}{c}\text { Storage Density } \\
\text { at STP } \\
\left(\mathrm{g} / \mathrm{cm}^{3}\right)\end{array}$ & $\begin{array}{c}\text { Boiling Point } \\
\left({ }^{\circ} \mathrm{C}\right)\end{array}$ \\
\hline Bismuth (Bi) & 209.0 & 7.3 & 9.8 & 1564.0 \\
Cadmium (Cd) & 112.4 & 9.0 & 8.7 & 767.0 \\
Cesium (Cs) & 132.9 & 3.9 & 1.9 & 671.0 \\
Iodine (I) & 126.9 & 10.5 & 4.9 & 184.3 \\
Krypton (Kr) & 83.8 & 14 & 3.7 & -153.4 \\
Xenon (Xe) & 131.3 & 12.1 & 5.9 & -108.1 \\
\hline
\end{tabular}

TABLE 1.1: Characteristics of propellant candidates discussed in this work.

Xenon has been adopted as a standard propellant of choice for many thrusters because of its attractive qualities and performance: $[11,12]$ xenon is relatively heavy, has a relatively low ionization threshold, and is inert. However, xenon is not abundant in Earth's atmosphere and is considerably more expensive than other propellants [13]. Jacobson et al. investigated krypton as a viable HT propellant and found that under certain conditions it performed comparably to xenon, but plasma termination occurred for high-voltage, high-current density operation with no known cause [14]. Heavy metals are expected to be good candidates for propellant, but propellant-induced short circuits are an issue for these propellants [13]. Grishin et al. investigated bismuth as a propellant candidate because of its enticing properties, i.e., relatively large atomic mass, relatively low price, and low ionization potential [15]. However, it has a considerably higher melting and boiling point than other candidates. The boiling point of bismuth is several hundred degrees $\mathrm{C}$, introducing difficulties when integrating gas feed systems on a spacecraft [16]. Cadmium provides an alternative to bismuth and was considered by Tverdokhlebov et al. [17] Cadmium has a lower boiling and melting point than $\mathrm{Bi}$, but is much more expensive, has low atomic mass, and is highly toxic in vapor form. Therefore, cadmium has been avoided [18]. Kieckhafer et al. outlines cesium as a propellant since it has comparable properties to xenon, but has only been suggested as a propellant as its cost is prohibitive and it is volatile around water [18]. Magnesium was investigated as a propellant by Hopkins and it was found that, while magnesium as a propellant has some advantages, significant challenges were encountered. Magnesium produced a more divergent plume with less energetic ions. Additionally, future designs for HT using magnesium would require extensive magnetic 
shielding [19]. Among the gas propellants discussed here, i.e., xenon and krypton, propellant storage and availability must be considered. These two gases require bulky compressed gas systems, adding undesirable payload mass and volume [20].

The latest propellant to be considered for HTs is iodine. Iodine has been explored, modeled, and tested in HTs and has proven to be a viable propellant [13, 17, 21, 22]. Iodine exists as a solid at room temperature and is easily sublimated at low temperatures. This attractive property eliminates the need for compressed gas systems that xenon and krypton require. Instead, gas storage systems that conform to the spacecraft structure are possible, eliminating designs that allocate specific space on the spacecraft for propellant storage. Iodine has comparable mass to xenon, has a lower ionization potential, and is much more abundant than xenon [20]. Implementation of iodine as a propellant is not without difficulties. As outlined in several works, atomic iodine, a halogen, is corrosive to many materials, which is a major concern for spacecraft integrity and operation $[17,20,21]$. Additionally, iodine is known to be toxic making it less desirable than other propellants. As a molecular gas, some of the applied power is coupled into rotational and vibrational transitions instead of ionization, reducing the power efficiency of HTs [23]. Hiller studied iodine as a propellant in a HT and found that the performance was comparable to that of xenon and even exceeded Xe performance in some cases, though additional engineering would be needed due to iodine build-up and corrosion [21]. A benefit observed in Ref. [21] is that, while some of the efficiency may be sacrificed when using iodine, the plume is less divergent and as a result there is less momentum loss compared to xenon. Since iodine naturally occurs as a diatom, a portion of the plume is expected to be singly ionized molecular iodine, providing nearly twice the thrust as xenon. However, just as a fraction of the propellant is theorized to be molecular, a fraction of the exhaust is expected to exist as negative ions. This is not desirable for HT since these ions are not magnetized and will be accelerated back into the HT, potentially damaging it [18]. Significant negative ion damage was not observed by Hiller, but spatially resolved ion density and flow measurement in the plume and channel, as well as, spatially localized magnetic field measurements, are identified as essential for future investigations of iodine HTs [21]. Limited optical access of the HT channel and the reactive nature of the plasma make diagnosing the plume difficult. Steinberger et al. have recently successfully measured singly ionized atomic iodine (I II) velocity distribution functions (VDF) using laser induced fluorescence (LIF) [20]. This technique opens a wide 
range of new possibilities to diagnose iodine plasma production, composition, and bulk flow non-perturbatively inside and outside a HT. Presented in this work are LIF measurements in an iodine plasma as a diagnostic technique for evaluating iodine bulk flows and determining magnetic field strengths from iodine spectra.

\subsection{Plasma Material Interaction and Fusion-relevant Plasmas}

Typically, fusion devices are divided into two categories: magnetically confined [24] and inertially confined [25]. An in-depth discussion of inertial confinement fusion is outside the scope of this work and is only mentioned here for completeness. A significant challenge to the future of both kinds of fusion devices is the integrity of the plasma facing components (PFC) [26]. The viability of fusion as a long-term source of energy hinges upon the lifetime of the materials used for the walls of the device [27]. Large heat flux is expected to be delivered to plasma facing components in fusion devices $\left(\sim 1 \mathrm{GW} / \mathrm{m}^{2}\right.$ for outer divertor in ITER [28]) and careful understanding of plasma dynamics is essential for mitigating damage [29]. Fusion wall materials must be able to withstand not only the high heat load induced by such plasmas, but also bombardment from plasma species (i.e. D, T, He, and neutrons) [26]. A particular area of concern for plasma induced damage is in the divertor region of a tokamak [27]. It is in this region that the magnetic field is carefully shaped to control the plasma exhaust and interactions with the wall. Figure 1.4 shows a simplified magnetic field geometry in a tokamak-like arrangement. In the divertor region, the magnetic field lines are directed to the divertor plate, a portion of the fusion device wall that is made of a different material that is less susceptible to plasma-induced damage. The unique field geometry in this region is designed to direct the plasma near the separatrix away from the core and also allow fusion by-products to be expelled. Plasma that exists near the separatrix is the most at risk of escaping confinement and reaching the reactor walls. Therefore all open field lines terminate on the divertor plate to protect the chamber walls [28]. The region between the last closed field line and the chamber wall is called the scrape-off layer (SOL) and this is a region of typical PMI.

Entire dissertations have been devoted to PMI for PFC to apply to fusion-relevant plasmas (Ref. [28] for example), though this work focuses on the contribution of neutral particles to fusion and fusion-relevant plasmas. Galante outlines major impacts of neutral gas to the fusion process: 


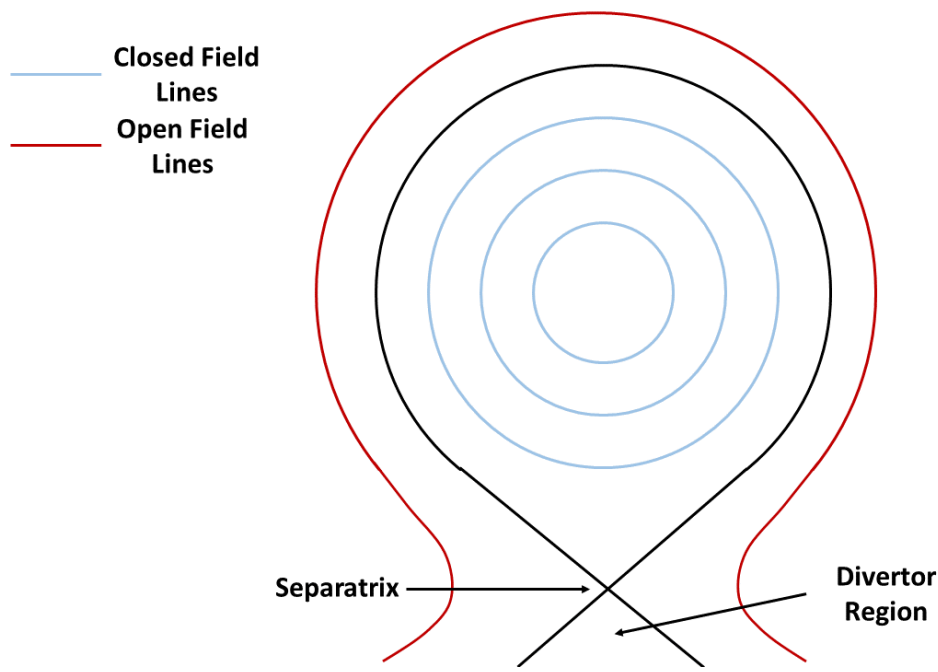

FiguRe 1.4: Simplified magnetic field geometry showing closed field lines (blue), open field lines (red), and the separatrix (black). Divertor region is indicated. The separatrix serves to direct unconfined plasma away from chamber walls to mitigate damage.

(1) charge exchange between neutrals and ions (2) the transition to high confinement mode (Hmode) and (3) increased retention and recycling from walls [30]. Measurements investigating the roles of neutrals in these different processes are difficult and the physics is not well understood.

\subsubsection{Charge Exchange}

Charge exchange occurs between a "cold" neutral and an energetic ion. During the collision, the ion is neutralized and the neutral particle is ionized, creating an energetic neutral and a "cold" ion:

$$
\mathrm{D}^{+}+\mathrm{D} \rightarrow \mathrm{D}+\mathrm{D}^{+}
$$

This process can be detrimental for the fusion process because the magnetic field structure of a fusion device is designed to confine charged particles. The now energetic neutral particles are not bound by the magnetic field and are free to carry energy away from the core of the fusion plasma [31]. Bombardment from the newly created energetic neutral particles erodes reactor walls and sputters material into the plasma. These two processes can decrease plasma heating and confinement [32]. The reverse process is equally important. Energetic neutrals injected as neutral beams convert to ions, injecting energy, momentum, and fuel into the plasma. Cold neutrals penetrating from the edge to the core of the plasma are also possible [30]. If too many neutrals are collecting at the core, confinement and efficient energy production may be hindered. The distance the neutrals can reach into the core is known as the penetration length. The more 
energetic a neutral, the further it can penetrate [32]. The penetration length is given by

$$
\lambda_{\text {pen }} \sim \frac{v_{n}}{n_{e}\langle\sigma v\rangle_{\text {ion }}}
$$

where $\lambda_{\text {pen }}$ is the penetration length, $v_{n}$ is the neutral velocity, $n_{e}$ is the electron density, and $\langle\sigma v\rangle_{\text {ion }}$ is the ionization rate [30]. Charge exchange between neutral and ionic hydrogenic species for fusion-relevant temperatures is expected to have the largest rate among all processes for hydrogen (see Fig. 1.4 of Ref. [30]) and therefore needs to be carefully considered for such plasmas.

\subsubsection{Role of Neutrals in the L-to-H Mode Transition}

A major discovery in the early 80 s was the first L-to-H mode transition (low-confinement mode to high-confinement mode) [33]. L-mode is typically characterized by poor confinement of the plasma and inefficient ionization, whereas H-mode shows a tremendous increase in confinement and a sharp decrease in the neutral population [30,34]. H-mode is commonly achieved in most fusion plasma devices today with the aid of auxiliary heating mechanisms and divertors [30]. Hmode is normally identified by a steepening in the plasma density and temperature profiles near the edge of the plasma. This is a signature of a transport barrier. These steep ion temperature and density gradients exist near the scrape-off layer (SOL) and are otherwise not seen in L-mode plasmas. This region of steep gradients is also referred to as the "pedestal" and pedestal physics is an area of active research [35]. The transport barrier reduces energy loss from the core of the plasma [34]. However, the physics in this region, specifically interactions with neutrals, is not well understood and models require accurate measurements of parameters to correctly predict fusion processes globally throughout the plasma. Hubbard states that reliable predictions and models for fusion devices are sensitive to the assumed neutral density values in the pedestal region and high resolution diagnostics in this region are essential [35]. Additionally, while the transport barrier reduces the amount of charge exchange processes that occur at the edge and divertor region, the plasma that does make it to the walls has a much higher ion temperature and induces more sputtering through charge exchange losses [34].

A common technique of determining the L-to- $\mathrm{H}$ mode transition is to observe the $\mathrm{D}_{\alpha}$ lineof-sight neutral emission-a drastic decrease in observed emission is indicative of an L-to-H 
mode transition [34, 36]. However, spontaneous emission measurements typically only return line-integrated values of the neutral emission and additional information is required to extract temperature and density information of neutrals from the $\mathrm{D}_{\alpha}$ measurements. Figure 1.5 shows the decrease in $\mathrm{D}_{\alpha}$ emission as a function of time observed in the transition from L-mode to $\mathrm{H}$ mode on DIII-D. Furthermore, investigations of L-to-H mode transitions with different divertor

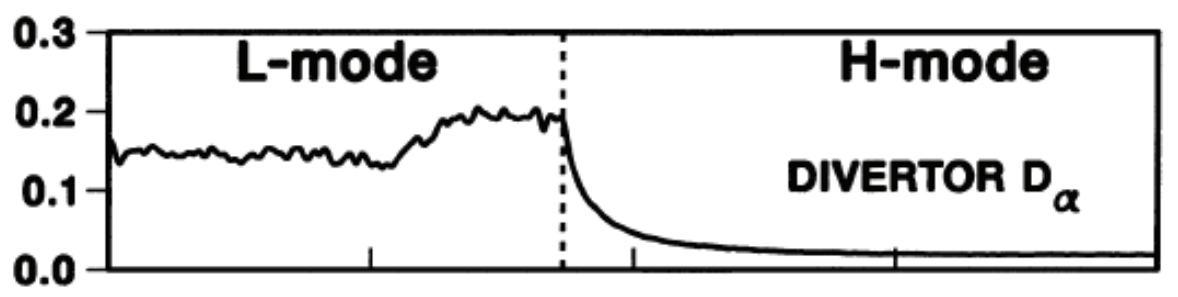

FiguRe 1.5: $\mathrm{D}_{\alpha}$ emission as a function of time observed during $\mathrm{L}-\mathrm{to}-\mathrm{H}$ mode transition. A drastic decrease in emission is observed and is indicative that the transition has occurred. Adapted from Ref. [36]. ㄷ IOP Publishing. Reproduced with permission. All right reserved.

configurations on the Experimental Advanced Superconducting Tokamak (EAST) stress that neutral density near the edge of the tokamak is critical for low power transition thresholds for L-to-H mode transitions [37]. During these experiments, neutral density was estimated from $\mathrm{D}_{\alpha}$ emission and accurate values are often left as one of the most important unknown quantities, highlighting the need for spatially localized diagnostics that measure neutral densities in fusion devices, specifically near the edge.

Recently, a new confinement mode has been predicted and observed on several large fusion devices, coined as "Super H-mode" (SH), that shows better confinement and performance than regular H-mode [38]. Super H-mode appears to be correlated with a branching in the pedestal pressure. The pressure of the pedestal dictates the transition to SH. Figure 1.6 shows the bifurcation of pedestal pressure as a function of pedestal density (reproduced from Ref. [38]). Figure 1.6 shows that if the pedestal density never reaches a sufficiently low value, the system tends to always relax to $\mathrm{H}$-mode. However, $\mathrm{SH}$ is transitioned to by beginning with a low pedestal density which is then increased. While the bifurcation is observed in global density and pressure values, diagnostics specifically probing the pedestal region with good spatial resolution are difficult and rare. Therefore, there is a need for such diagnostics that can measure the neutral density in the edge of the fusion devices up to the separatrix. 
EPED Predictions Compared to DIII-D Observations (171322)

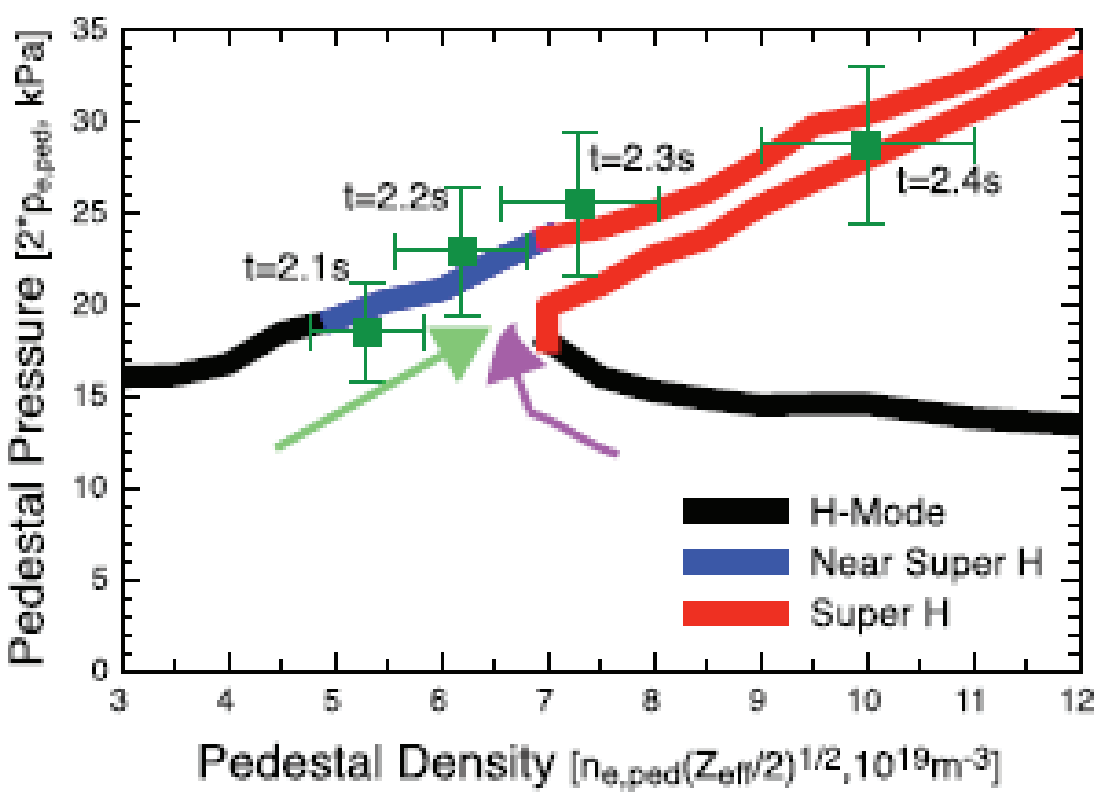

FiguRE 1.6: Bifurcation in pedestal pressure as a function of pedestal density that facilitates transition to SH (red) from H-modes (black). Measurements on DIII-D (green squares) are in good agreement with simulated regimes. IAEA Publishing. Reproduced from Ref. [38] with Permission. All rights reserved.

\subsubsection{Neutral Recycling and Retention}

Lastly, fusion performance is heavily dependent on neutral retention in the walls and recycling of neutrals from the walls. As mentioned previously, ions that make it past the last closed magnetic field surface can participate in charge exchange. Ions that reach the edge can also be neutralized and embedded into the chamber wall only to be released at a later time from sputtering. Some fusion reactor walls are designed with neutrals already deposited into them to be released into the plasma as a secondary source of fuel (recycling) [39]. However, the amount of neutral particles that are emitted must be carefully monitored since rapid release of these particles leads to density limited events within the plasma and can damage the chamber [30]. As a result, the material composition of the wall [40] and methods to identify retention and recycling of neutrals are pivotal to the success of fusion reactors.

\subsection{Neutral Particle Diagnostic Techniques}

As stated above, many common diagnostics that probe neutral populations lack spatial localization, making these techniques less suitable to determine how neutral dynamics change on small 
scales. Commonly, optical emission spectroscopy (OES) is implemented for neutral dynamics, but this method is line-integrated and requires rate equations based on electron temperatures and densities to extract useful quantities such as neutral temperature or density. Edge-mounted pressure gauges provide neutral gas pressure, from which density is calculated using the ideal gas law. However, typically pressure gauges are situated far from the plasma and require assumptions about the spatial profile of the neutral density and temperature. Yu et al. developed an in-situ laser spectroscopic technique to determine composition of PFC components, but the technique lacks the ability to reveal species composition and neutral densities between the chamber wall and separatrix [41]. The importance of localized measurements of neutral particle characteristics cannot be overstated. Hubbard emphasizes the importance of developing a diagnostic that is capable of measuring plasma (and neutral) dynamics in the edge of the fusion devices for the validation of transport models [35]. The Report of Research Needs Workshop has also outlined several high-priority items for the future of fusion, many of which are intimately linked to the role of neutrals in the plasma edge (p. $86-87,90)$ : [42]

"Finding solutions to the core, pedestal, and divertor will require the understanding of significantly different plasma physics regimes, ranging from the hot, low collisionality core plasma, to the open magnetic field line dominated scrape-off layer, and the high plasma and high neutral density divertor."

"Particle transport issues that relate to the core-edge coupling include the removal of helium ash from the core plasma through the pumped divertor, the cycling of impurities (both intentional and unintentional) and fuel between the core plasma and plasma material interfaces, particle retention in the solid materials, and the particle behavior in the presence of high power transfer to the solid materials."

"The materials are affected by possible melting, fast ion or electron impingement, neutron damage, tritium and deuterium retention, and erosion and redeposition by plasma. The impact on the core plasma arises primarily from the generation of impurities, dust, and larger material removal such as bubbles and flakes."

"New measurement requirements include profile, flow and fluctuation measurements with better geometric coverage; measurements of Ly- $\alpha$ and other radiation 
sources in dense divertor plasmas to account for radiation transfer and opacity effects; and neutral density in the divertor, SOL and edge pedestal to understand fueling and divertor performance."

In this work, another laser-based technique to investigate neutral properties in fusion and PMI plasmas is described. Two-photon absorption laser induced fluorescence (TALIF) provides highly localized measurements of ground state neutral hydrogen isotope density and is ideal for fusion plasma applications [43]. Presented in this work are TALIF measurements of neutral deuterium conducted in a fusion-relevant plasma.

\subsection{Summary}

Two plasmas are investigated in this work, Hall thruster-relevant and fusion-relevant plasmas. Velocity distribution functions are easily accessible in both systems using laser-based fluorescence techniques. Specifically, singly ionized atomic iodine velocity distribution functions are measured for future Hall thruster diagnosis using LIF and neutral velocity distribution functions are measured in Proto-MPEX in fusion-relevant regimes using TALIF. For both Hall thrusters and fusion plasmas, measurements of species flow, density, and interaction with device walls is critical and rarely measured with spatial resolution and non-perturbatively. The measurements reported here show that the techniques used in this work are able to successfully provide nonperturbative, spatially localized measurements of ion bulk flow in the channel and plume of a HT and neutral densities, flows, and temperatures in the edge of fusion devices. 


\section{Chapter 2}

\section{Single and Multi-photon Laser}

\section{Induced Fluorescence}

\section{$2.1 \quad$ Introduction}

Optical and laser-based diagnostics are ideally suited to perform non-perturbative measurements of a variety of field and particle properties in plasmas. Passive optical emission spectroscopy (OES) has regularly been employed to measure electron temperature, plasma density [44], and reduced electric field [45]. Single-wavelength lasers have been used to perform Thomson scattering (electron temperature, electron bulk flow, electron density measurements) [46], cavity ring-down spectroscopy (CRDS) (species concentration measurements) [47], laser induced breakdown spectroscopy (LIBS) (material composition measurements) [48], digital holography (DH) (surface topology measurements) [49], and Faraday rotation (magnetic field strength, rotational band structure, and density measurements) [50]. Tunable frequency laser systems have been used for fluorescence techniques (relative density, thermal temperature, ion bulk flow measurements) [51, 52] and Zeeman splitting-magnetic field correlation measurements [53]. While this list of optical and laser techniques is not exhaustive, it provides insight into the versatility of laser-based diagnostics.

Commonly, laser techniques rely on the scattering or absorption of a specific wavelength by the target species, i.e., atoms, ions, or electrons. Specifically, this work highlights the abilities of LIF to investigate both neutral and ionic species in unique plasma systems. Single-photon 
LIF is used to measure I II flow speeds; TALIF is used to measure absolute neutral densities in deuterium using krypton and xenon as calibration gases; and 3pLIF schemes are proposed for neutral krypton.

LIF schemes often exploit metastable states to ensure a sufficient target species population density exists. However, metastable states are not always directly indicative of the ground state behavior and for many species, appropriate metastable states do not exist. For this reason, it is desirable to probe ground state transitions directly. Because of the large energy spacings required, TALIF is often used to investigate ground state transitions. Recently, a new approach of using three photons has been explored, coined as 3pLIF [54]. Like LIF, a VDF obtained from TALIF or $3 \mathrm{pLIF}$ provides the thermal temperature, bulk flow, and relative density. However, because these multi-photon approaches probe ground state transitions, they can also provide measurements of target species density. In a multi-photon scheme, the photons are absorbed nearly simultaneously, each providing a fraction of the required energy for the transition. In reality, there exists a finite time between the absorption of each photon $(\sim 10 \mathrm{fs}$ for TALIF) [30]. The first photon excites the electron to a "virtual" state [55]. In TALIF, the second photon "completes" the transition, allowing the electron to enter an energy level otherwise inaccessible due to classical angular momentum addition rules. In 3pLIF, the second photon excites the electron to a second virtual state and the third photon completes the transition.

\subsection{Laser Induced Fluorescence}

\subsubsection{Theory}

As a diagnostic technique, LIF has become a standard method to investigate bulk flow, temperature, and relative population density since it was first developed in 1975 [51]. LIF is a method in which the VDF of an atom or ion is measured by exciting an electronic transition with laser radiation and collecting the fluorescence of the excited state. The Doppler motion of a particle causes slight changes in the absorption frequency for the electronic transition. Typically, a narrow bandwidth laser (commonly in or near the visible spectrum) is used to probe a species in a localized region of a plasma. By sweeping the frequency of the laser, the VDF is measured 
by relating the spectral shift in the absorption frequency to the velocity of the absorber:

$$
v=\frac{f-f_{\circ}}{f_{\circ}} c
$$

where $v$ is velocity, $f$ is the laser frequency, $f_{\circ}$ is the rest frame frequency, and $c$ is the speed of light.

To eliminate problems related to scattered light from the probing laser, the collected signal is typically at a different frequency than the stimulating radiation-scattered light issues are notoriously problematic for other laser-based diagnostics e.g., Thomson Scattering. While LIF typically provides measurements of the relative density of the state being interrogated, LIF may be absolutely calibrated if reliable rate equations are available for the probed transition and the local electron temperature is known. Rayleigh scattering is a common technique used to determine the unknown quantities in the rate equation [56]. However, calibration is not commonly attempted for single-photon LIF, as it is difficult in theory and tedious in implementation. In theory, the amount of collected signal is dependent on many factors, but in practice it is most affected by the laser intensity and the number of available absorbers. A model of integrated collected signal is provided by Amorim et al. highlighting the strong dependence on the laser intensity and target density:

$$
\mathrm{I}_{\mathrm{LIF}}=K B_{1-2} I_{\mathrm{las}} n \frac{A_{2-3}}{Q_{2}+A_{2}},
$$

where $\mathrm{I}_{\mathrm{LIF}}$ is the integrated LIF intensity; $K$ is a factor that accounts for detector efficiency, gain, and transmission; $B_{1-2}$ is the Einstein absorption coefficient for the target state to the excited state ( 1 and 2 respectively); $I_{\text {las }}$ is the laser intensity (sometimes expressed by number of photons using $E / h \nu) ; n$ is the density of the target state; $A_{2-3}$ the Einstein spontaneous transition probability between the excited state and relaxed state ( 2 and 3 respectively); $Q_{2}$ is the collisional depopulation factor for the excited state; and $A_{2}$ is the Einstein spontaneous transition probability for the excited state [56]. It can be seen from Eq. 2.2 that several terms are not readily known and must be independently determined for an absolute density calculation.

A subtle omission from Eq. 2.2 is a dependence on the functional form of the measured lineshape. The measured lineshape is a convolution of the Doppler-broadened lineshape and the laser 
lineshape (ignoring all other broadening effects):

$$
g(\Delta \nu)=\int_{-\infty}^{\infty} d \tau f_{\mathrm{G}}(\tau) f_{\mathrm{las}}(\Delta \nu-\tau)
$$

where $g(\Delta \nu)$ is the measured lineshape, $f_{\mathrm{G}}$ is the Doppler lineshape, and $f_{\text {las }}$ is the laser lineshape. If both the Doppler and laser lineshape are Gaussian (for single-photon LIF, a suitable approximation), the measured lineshape will have a resultant width, $\gamma_{\text {meas }}$ that is the quadrature sum of the Doppler width $\gamma_{\mathrm{D}}$ and the laser linewidth $\gamma_{\text {las }}$ (App. A):

$$
\gamma_{\text {meas }}=\sqrt{\gamma_{\mathrm{D}}^{2}+\gamma_{\text {las }}^{2}}
$$

The measured amplitude of signal is, in addition to the factors above, inversely proportional to Eq. 2.4. For very large laser linewidth (as a result of either physical limitations of the laser system or degraded operation) or very large Doppler width (i.e., very "hot" population) the measured amplitude decreases. However, this factor is commonly left out of many signal models since the models, e.g., Eq. 2.2, describe integrated signal and not the signal amplitude at a specific wavelength. In other words, the inverse relationship of the width of the measured profile decreases the amplitude because of an increase of broadening in the measured lineshape (either by Doppler broadening or increased laser linewidth), an effect that is accounted for in the integration. ${ }^{1}$

For the experiments reported here, Doppler broadening dominates the measured lineshape for single-photon LIF measurements. In the zeroth-order case, a Doppler-broadened lineshape is Gaussian, and, as such, the lineshape is simply fit to a Gaussian and the fit parameters provide the plasma quantities,

$$
f_{\mathrm{G}}=a+b \exp \left(-\frac{m_{s}\left(v-v_{\circ}\right)^{2}}{2 e T_{s}}\right),
$$

where $a$ is a factor that accounts for a DC offset introduced by the detecting electronics, $b$ is the intensity of the measured signal, $m_{s}$ is the mass of the species being probed (in $\mathrm{kg}$ ), $v$ is velocity (derived from converting the span of laser frequencies to a speed using Eq. 2.1), $v_{\mathrm{o}}$ is the bulk flow, $e$ is the elementary charge (necessary for direct output of temperature in $\mathrm{eV}$ ), and $T_{s}$ is

\footnotetext{
${ }^{1}$ It is common for the measured lineshape to be dominated by Doppler-broadening, i.e., the measured lineshape is Gaussian. Therefore the integrated signal is proportional to the product of the amplitude $A$ and the measured width. This effect states that as the amplitude decreases, the width increases and is offset in the integration.
} 
the species thermal temperature in $\mathrm{eV}$. Mathematically, the species temperature is related to the FWHM of the lineshape, the bulk flow is the mean offset of the lineshape, and the relative density is determined from the area under the lineshape. When computing the relative density from a measured lineshape, it is important to remove the DC offset from Eq. 2.5. However, the above discussion is only true if (1) thermal broadening is the dominating source of broadening or (2) other comparable sources of broadening have been removed from the lineshape. A detailed discussion of broadening mechanisms is presented in Chapter 3.

If the measured lineshape does not conform to a Gaussian lineshape, the same quantities can be calculated from the moments of the lineshape. The average relative density, average bulk flow, and average thermal temperature are given by the zeroth, first, and second moments, respectively, of the measured velocity distribution function. Mathematically, these are shown below; the first and second moments are normalized to the zeroth moment (density), and the second moment is taken in the frame of the absorber.

$$
\begin{gathered}
\langle n\rangle=\int_{-\infty}^{\infty} f(v) d v \\
\langle v\rangle=\frac{\int_{-\infty}^{\infty} v f(v) d v}{\int_{-\infty}^{\infty} f(v) d v} \\
\frac{1}{2} m\left\langle v^{2}\right\rangle=\frac{\frac{1}{2} m \int_{-\infty}^{\infty}(v-\langle v\rangle)^{2} f(v-\langle v\rangle) d(v-\langle v\rangle)}{\int_{-\infty}^{\infty} f(v) d v}
\end{gathered}
$$

\subsubsection{LIF Transition Specifics}

Generally, single-photon LIF systems use a narrow bandwidth, continuous-wave (CW) laser to perform spectroscopic measurements, though it is not uncommon to perform such measurements with a pulsed laser system. In this work, a Sirah Matisse DR narrow linewidth, tunable dye laser was used to probe I II. Figure 2.1 shows a partial Grotrian diagram, first proposed by Hargus et al., for the LIF scheme used in this work [22]. The metastable ${ }^{5} \mathrm{D}_{4}^{\mathrm{o}}$ state is excited to the ${ }^{5} \mathrm{P}_{3}$ state with $696.0694 \mathrm{~nm}$ photons [20]. Fluorescence is observed at $516.264 \mathrm{~nm}$ as the 


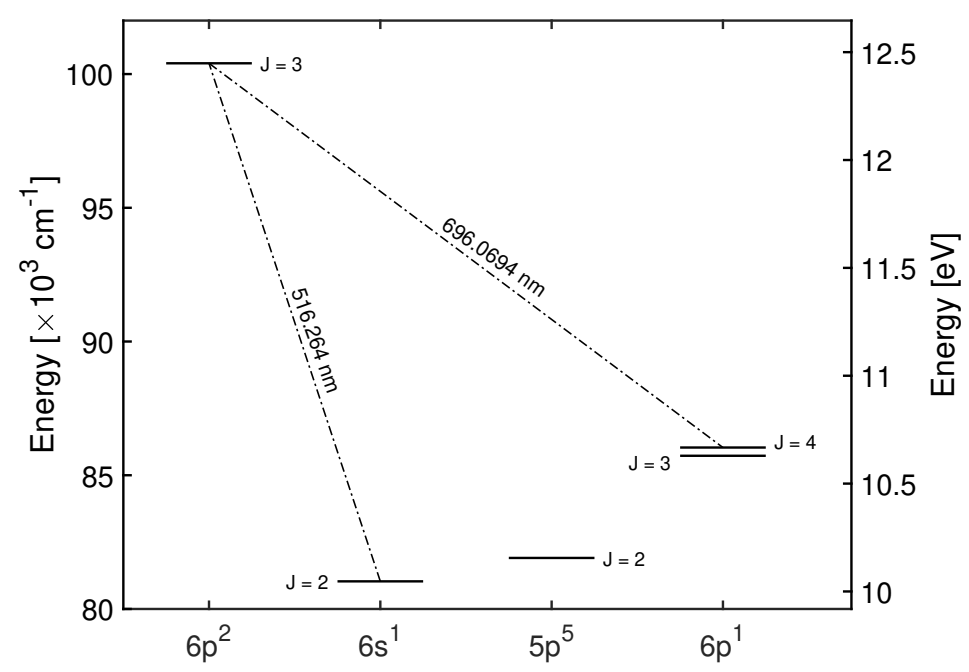

FiguRe 2.1: Partial Grotrian diagram of the I II transition probed in this work (described in text). Wavelengths are listed in vacuum for all cases. Reproduced from Ref. [20]; reprinted by permission of the American Institute of Aeronautics and Astronautics, Inc.

${ }^{5} \mathrm{P}_{3}$ state relaxes to the ${ }^{5} \mathrm{~S}_{2}^{o}$ state. It is important to mentioned that Martin et al. previously reported the pump transition at $696.070 \mathrm{~nm}$, but Steinberger et al. observed the transition with higher precision and found it to be $696.0694 \mathrm{~nm}[20,57]$. While the two values agree within experimental error, if the value used in Ref. [57] is used, a static I II plasma will appear to have a bulk flow. Discrepancies in this measured value are likely due to the significant hyperfine splitting of the probed transition. Wavelengths are quoted in vacuum for all cases.

Hyperfine splitting occurs when the spin angular momentum of a particle's nucleus interacts with the total electronic angular momentum. In some cases, several hyperfine splits of the energy state are observed and many transitions within a very small spectral window can occur. For the transition selected for this work, there exist 15 allowed hyperfine transitions that contribute to the overall measured lineshape. The details of hyperfine splitting of iodine transitions are given in Sec. 3.5 and the measured lineshape is shown is Sec. 5.2.

\subsection{Two-photon Absorption Laser Induced Fluorescence}

\subsubsection{Theory}

Multi-photon processes are sometimes more desirable as they have the ability to probe electronic states that are otherwise inaccessible by standard single-photon LIF. There are significant differences between the two techniques, such as dependence on the laser intensity (energy). For 
single-photon LIF, the signal intensity is proportional to the laser intensity (Eq. 2.2). However, TALIF signal intensity has a quadratic dependence on laser intensity. The squared dependence is a result of two quanta of light being absorbed rather than one in conventional LIF schemes [58]. The squared dependence on laser intensity provides increased spatial localization compared to LIF since there is less absorption along the laser path because the intensity of the laser light is only sufficient to excite the two-photon transition near the focal spot of injected light [30].

The two-photon absorption profile has been outlined in several other works, all with subtle differences in notation [43, 59-62]. In the interest of clarity, this work will employ the notation used in Ref. [59]:

$$
\mathrm{I}_{\mathrm{LIF}}=n \frac{\Omega}{4 \pi} \eta T G_{\mathrm{TP}} \sigma_{\mathrm{TP}}^{(2)}\left(\frac{E}{h \nu}\right)^{2} g a,
$$

where $\mathrm{I}_{\mathrm{LIF}}$ is the integrated signal (photons $\cdot s \cdot \mathrm{m}$ ), $n$ is the density of the interrogated species $\left(\mathrm{m}^{-3}\right), \Omega / 4 \pi$ is the solid angle of collection normalized by the solid angle of a sphere, $\eta$ is the unitless quantum efficiency of the photodetector at the fluorescence wavelength, $T$ is the transmission of optical components, $G_{\mathrm{TP}}$ is a statistical factor that arises from the nature of an electron absorbing two photons simultaneously and has a value of 2 [63], $\sigma_{\mathrm{TP}}^{(2)}$ is the two-photon absorption cross section $\left(\mathrm{m}^{4} \cdot \mathrm{s} \cdot\right.$ photons $\left.^{-1}\right), E$ is the laser energy per pulse $(\mathrm{J} / \mathrm{pls}), h \nu$ is the energy per photon $(\mathrm{J} /$ photon $), g$ is the gain of the detecting system at the applied voltage of the photodetector, and $a$ is the branching ratio for the interrogated transition fluorescence. The gain and quantum efficiency of the detector are determined from the curves supplied directly by Hamamatsu, shown in figures 4.19 and 4.20. Similar to the signal equation for single-photon LIF, the amplitude of measured TALIF signal is dependent on the measured width. However, in this case the functional form of the measured width is more complex since the Doppler lineshape is Gaussian and the laser lineshape is Lorentzian. Details explaining why the laser linewidth for TALIF is Lorentzian are discussed in Chapter 4. For the measurements reported in this work, this factor is not included since the method of absolute density calibration uses the integrated signal (Eq. 2.7) and not solely signal amplitude.

Since the mechanism responsible for exciting the ground state transition is the absorption of two photons, several of the quantities in Eq. 2.7 are modified with respect to conventional models of single-photon LIF. One of the most noticeable differences is in the two-photon absorption 
cross section $\sigma_{\mathrm{TP}}^{(2)}$. This particular cross section is often reported in the units "GM" (GöppertMayer-1 GM $=10^{-50} \mathrm{~cm}^{4} \mathrm{~s}^{1}$ photons $^{-1}$ ) named after Maria Göppert-Mayer, who first estimated the two-photon absorption cross sections in 1931 [64], though the confirmation of her theory wouldn't come until decades later with the invention of the laser [52]. The units for $\sigma_{\mathrm{TP}}^{(2)}$ reveal important physics for the process of TALIF. First, the distance to the fourth power dependence arises from the cross section of each photon that must collide with the target species. A crude analogy useful in interpreting this feature is that a cross section is related to the probability that a collision will occur. If two photons need to collide with an ion then the probability that this would occur is the product of the two single-photon collisions. However, these collisions must occur on a specific time-scale. The two photons must collide nearly simultaneously with the target for the TALIF transition to be successful. Thus, there is a time-scale in the units for the two-photon cross section.

Some values included in Eq. 2.7 do not need to be directly measured because Eq. 2.7 is only used when comparing cross sections to another species, usually for calibration. As a result, the terms that arise from the collection apparatus (namely the solid angle of collection and optical transmission of components) are common to all measurements and independent of interrogated species. This is a fortunate consequence because, in many cases, it is fairly difficult to accurately determine these quantities.

Parameters measured by LIF, in many cases, can be measured globally with other diagnostic techniques. For example, bulk flow of charged species is sometimes measured using a Mach probe $[65,66]$. However, such techniques commonly lack non-perturbative, in-situ, or spatially localized capabilities and are not discussed further.

In this work a pulsed laser system is used (Sec. 4.2.1) to measure neutral velocity distribution functions (NVDFs) via TALIF. From this, absolute densities, bulk flows, and neutral temperatures are determined.

\subsubsection{TALIF Transition Specifics}

As mentioned in Sec. 2.1, two-photon schemes require energetic photons to excite ground state transitions. A consequence of absorbing two photons is a drastic decrease in the absorption cross section for the transition. It is for this reason that TALIF systems commonly employ a 
pulsed laser with many frequency conversion stages to create intense, short wavelength pulses. In this work, deuterium, xenon, and krypton are investigated, with the latter two species used as calibration gases. Figure 2.2 shows a partial Grotrian diagram for each of the specific schemes that are interrogated in this work. Table 2.1 lists the information presented in Fig. 2.2. In

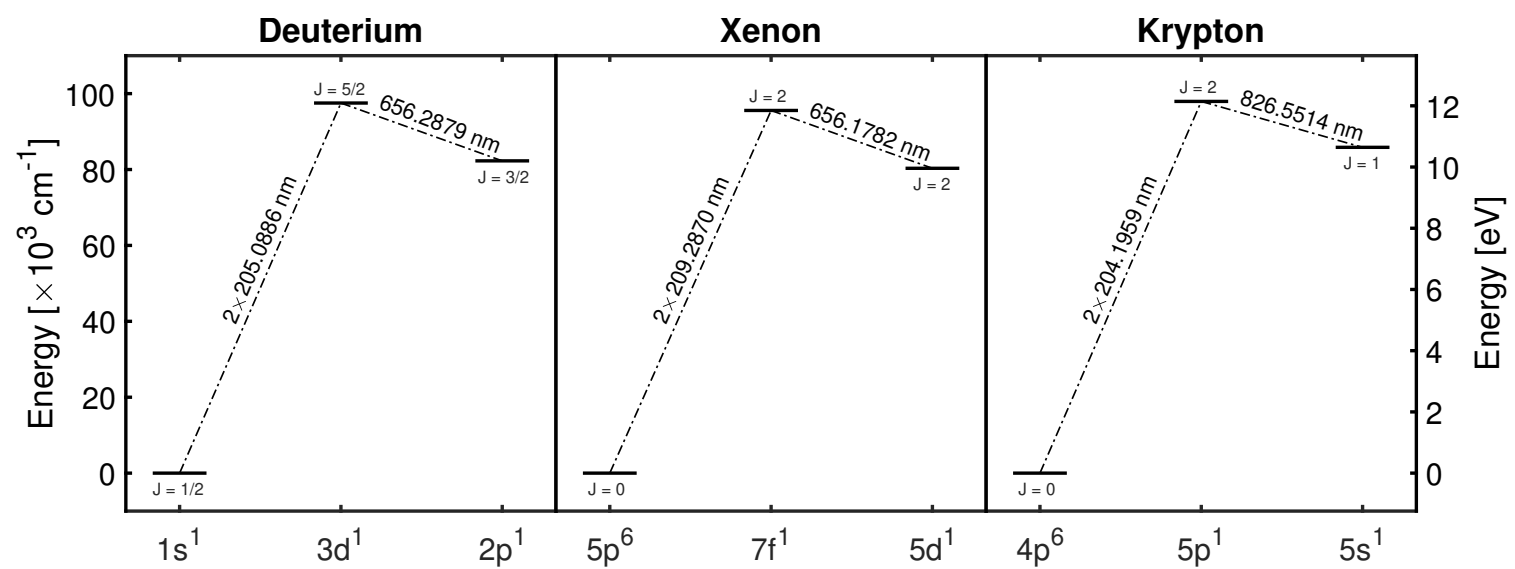

Figure 2.2: Partial Grotrian diagram for deuterium, xenon, and krypton. Energy levels for each state of each species are shown in $\mathrm{cm}^{-1}$ on the left axis and in $\mathrm{eV}$ on the right axis. Wavelengths are reported in vacuum for all cases. The pump wavelength is similar for deuterium and krypton. The fluorescence for these two species is very different, creating optimal optical alignment difficulties. Deuterium and xenon have notably different pump transitions, but nearly identical fluorescence wavelengths-making optimal optical alignment between the two species much easier.

Tab. 2.1, two different wavelengths are reported: first in the electron frame, i.e., the resonant wavelength needed to excite the ground state, and the second in the UV frame, i.e., the laser output wavelength.

\begin{tabular}{lcccc}
\hline \hline & Transition & Electron & UV & \\
Element & Scheme & Frame & Frame & Fluorescence \\
& $($ Racah $)$ & $\lambda_{12}$ & $\lambda_{12}$ & $\lambda_{23}$ \\
(nm vac.) & (nm vac.) & (nm vac.) \\
\hline $\mathrm{D}$ & $1 s \rightarrow 3 d \rightarrow 2 p$ & 102.5443 & 205.0886 & 656.2879 \\
$\mathrm{Xe}$ & $5 p^{6} \rightarrow\left({ }^{2} \mathrm{P}_{3 / 2}\right) 7 f \rightarrow\left({ }^{2} \mathrm{P}_{3 / 2}\right) 5 d$ & 104.6435 & 209.2870 & 656.1782 \\
$\mathrm{Kr}$ & $4 p^{6} \rightarrow\left({ }^{2} \mathrm{P}_{3 / 2}\right) 5 p \rightarrow\left({ }^{2} \mathrm{P}_{1 / 2}\right) 5 s$ & 102.0980 & 204.1959 & 826.5514 \\
\hline
\end{tabular}

TABLE 2.1: Transition and level information for each gas interrogated. Transition levels are given in the Racah notation. Vacuum wavelengths are given in all cases. Two different pump transitions $\lambda_{12}$ are presented, one in the "electron" frame (wavelength needed to excite an electron from the ground state) and the "UV frame" (wavelength emitted from the laser-i.e., excitation wavelength multiplied by 2). The fluorescent wavelength $\lambda_{23}$ is given in the last column. 


\subsubsection{Absolute Density Calibration}

A powerful technique employed with most TALIF measurements is to calibrate the measured signal to determine the absolute density of the interrogated species. TALIF typically measures ground state transitions and calibration methods generally don't require extensive rate equations-therefore, it is much more common to absolutely calibrate TALIF diagnostic measurements. TALIF measurements are calibrated by many techniques, the appropriate method is dictated by the system and species studied. Common TALIF calibration techniques are resonant absorption spectroscopy (RAS) [56], resonant-enhanced multi-photon ionization (REMPI) [67], titration [68], and noble gas TALIF measurement comparison (the calibration method used in this work) [69]. Calibration by noble gas comparison was first suggested by Döbel et al. [69] He showed that krypton is a suitable calibration gas for hydrogen and deuterium. However, krypton is difficult to use for some systems because the fluorescence of deuterium occurs at 656 $\mathrm{nm}$, while the fluorescence for krypton occurs at $826 \mathrm{~nm}$. Using such a calibration gas requires optical re-alignment and a wide spectrum detector when probing both species.

For this reason, Elliott et al. determined the relative two-photon absorption cross section between krypton and xenon, providing a way to use xenon as a calibration gas for deuterium [61]. While the cost of xenon gas is far greater than krypton or other noble gases, the fluorescence wavelength for xenon is almost identical to that of deuterium, making optimal alignment for both species nearly the same. However, the excitation wavelength for xenon significantly differs from that for deuterium. Exciting the xenon transition used in this work requires adjusting the TALIF laser gratings to produce wavelengths near the edge of the laser's operational range. As a result, the energy per pulse produced at xenon excitation wavelengths is much less than that for krypton or deuterium. A final difficulty when using xenon as a calibration gas is the smaller absorption cross section compared to krypton, further diminishing the maximum collectible calibration signal [61].

To use Eq. 2.7 to calibrate a TALIF measurement, the signal is normalized to values unique to each species for a specific experimental configuration:

$$
S^{\prime}=\frac{S}{\eta\left(\frac{E}{h \nu}\right)^{2} g a}=n \frac{\Omega}{4 \pi} T G_{\mathrm{TP}} \sigma_{\mathrm{TP}} .
$$


In Eq. 2.8, $\Omega / 4 \pi, T$, and $G_{\mathrm{TP}}$ are all independent of target species. However, if krypton is used as a calibration gas, the collection optics require two different bandpass filters. The transmission term in Eq. 2.8, T, is split into two terms in this case: the transmission of the bandpass filters for the given species $T_{\text {Fil. }}$ and the transmission of the overall optical system $T_{\mathrm{Op}}$. That is, when krypton is used as a calibration gas, $T=T_{\mathrm{Fil}} \cdot T_{\mathrm{Op}}$. For completeness, the transmission of each filter was measured and is shown in Fig. 2.3. It is clear from Fig. 2.3 that there is essentially no difference between the transmission through the bandpass filter for the fluorescence of deuterium and xenon, but there is significant difference between the transmission of the krypton fluorescence compared to deuterium and xenon fluorescence. Additionally, as will be discussed in Chapter 6, measurements for deuterium and xenon required the use of multiple bandpass filters, decreasing the overall transmission of deuterium and xenon signal compared to krypton.

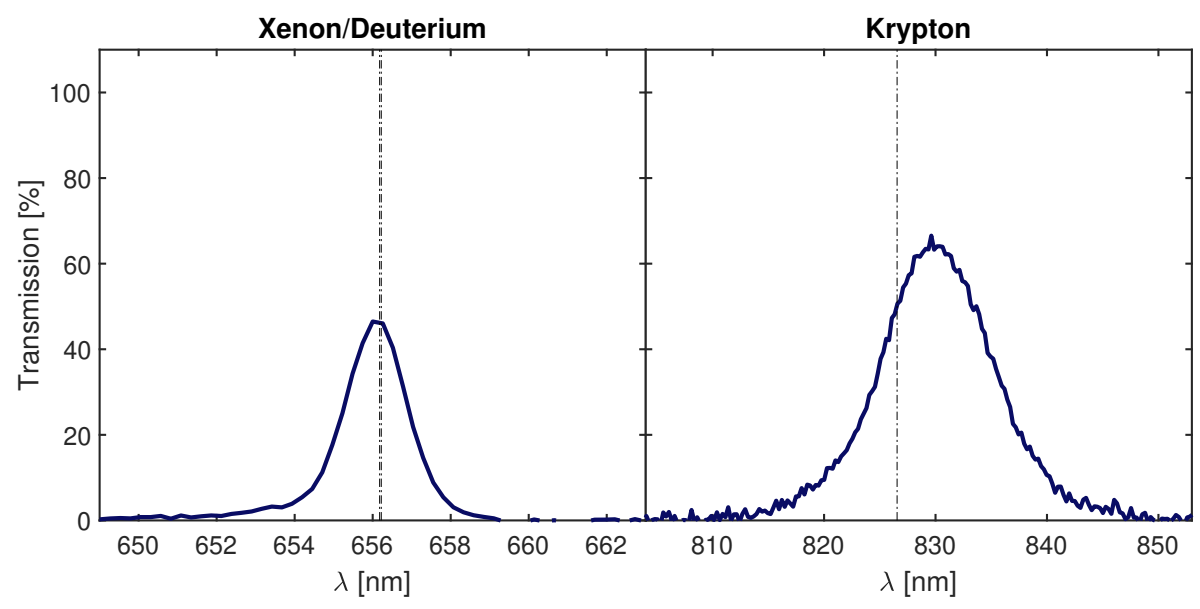

Figure 2.3: Measured filter transmission used for deuterium and xenon (left) and krypton (right). The black dot-dash line indicates the predicted fluorescence wavelength for each species. The fluorescence wavelength for xenon and deuterium are not spaced far enough apart to be distinguishable on this scale.

The ratio of two TALIF measurements provides the absolute density of the target species if the relative two-photon cross section between the two gases and the absolute density of the calibration gas are known. In most cases, the density of deuterium is calibrated with krypton. Niemi et al. found the relative cross section ${ }^{2}$ between hydrogen and krypton to be $\sigma_{\mathrm{Kr}}^{(2)} / \sigma_{\mathrm{H}}^{(2)}=$ 0.62 for the transitions used in their work [70]. When quoting these cross sections, it is common to include a superscript (2) that indicates that the cross sections are the two-photon cross

\footnotetext{
${ }^{2}$ Niemi et al. estimates that this value is correct within $50 \%$.
} 
sections. The relative cross section ${ }^{3}$ between xenon and hydrogen is given by Elliot et al. and has a value of $\sigma_{\mathrm{Xe}}^{(2)} / \sigma_{\mathrm{H}}^{(2)}=0.024$ [61]. The density of the calibration gas is given by the ideal gas law. To this end, it is crucial that the static fill of the chamber is monitored by a pressure gauge capable of accurately measuring the calibration gas pressure. The static fill pressure was measured by a suite of Baratron pressure gauges at different axial locations along Proto-MPEX for the measurements presented here. Proto-MPEX was typically filled with 100 mTorr of either xenon or krypton. The pressure gauge situated nearest to the TALIF measurement location was queried for the static fill pressure. Figure 4.21 in Sec. 4.2.6 shows the location of the TALIF measurements and the location of the pressure gauge used for calibration.

\subsection{Three-photon Absorption Laser Induced Fluorescence}

\subsubsection{Theory}

The ability to probe ground state and neutral populations has immense benefits when studying systems that rely heavily on behavior of neutrals. As highlighted above, TALIF provides a means to probe such species and provides localized measurements of thermal temperature, bulk flow, and absolute density. However, difficulties arise when using TALIF systems on large devices where the electromagnetic interference (EMI) near the vessel can be intense. Laser light in these environments is normally transmitted to the machine from far away $(>15 \mathrm{~m})$ where the electromagnetic interference (EMI) is low. Normally, laser light is fiber coupled over these distances solving the tedious and difficult problem of free-space transmission. However, it is often the case that TALIF schemes rely on UV light. Deep UV light cannot be coupled to a fiber over these distances, requiring large beamline structures and fine motor-controlled mirrors to deliver UV pulses to the plasma. Additionally, many common vacuum components and optics have low transmission percentages for UV light, introducing significant energy losses.

Recent developments in multi-photon laser induced fluorescence have successfully measured hydrogen velocity distribution functions in a combustion system using 3pLIF [54]. This advancement alleviates some of the difficulties associated with TALIF. First, a three-photon scheme employs light that is significantly closer to the visible spectrum, making transmission through

\footnotetext{
${ }^{3}$ The term cross section should be taken with some caution here as the value quoted in Ref. [61] is a relative calibration factor rather than a true relative cross section since many terms were grouped into the measurement of this number.
} 
standard vacuum components and optics much more efficient and allows for the use of cheaper materials. Second, since the laser light is closer to the visible spectrum, light is much more easily coupled to a high power fiber and attenuation will have less effect on the laser energy over long distances, eliminating the need for large beamlines. The ability to fiber couple light also eliminates the need for alignment of the confocal collection apparatus (Sec. 4.2.4) at the vacuum vessel. Rather, alignment can be completed away from the target machine and the confocal apparatus mounted at a later time.

The system studied by Jain et al. differs significantly from the system investigated in this work and, as a result, there is no guarantee that a 3pLIF scheme would be successful on fusionlike plasmas. However, to encourage future consideration, possible 3pLIF schemes for neutral krypton are presented here. Krypton was chosen as the test case gas because there exist twoand three-photon schemes without the need for the gas to exist as a plasma. A simple $\mathrm{Kr}$ reference cell suffices to make a proof-of-principle measurement. Second, the two-photon cross section for $\mathrm{Kr}$ is very large and the fluorescence is significant, even at very low laser energies. Therefore, krypton is an attractive 3 pLIF test case.

The selection rules for a three-photon process to occur are similar to that for two-photon. The total angular momentum $J$ for each photon process must change by \pm 1 . Therefore, transitions that have a total angular momentum change between states of \pm 1 or \pm 3 are allowed. State information for theorized krypton 3pLIF schemes is outlined in Sec. 2.4.2. Since the ground state of neutral $\mathrm{Kr}$ has angular momentum $J=0$, only states that have $J=1$ or $J=3$ are allowed.

Generally, for multi-photon laser induced fluorescence, the LIF signal is proportional to the laser intensity raised to the $n^{t h}$ power, where $n$ is the number of photons, $\mathrm{I}_{\mathrm{LIF}} \propto I_{\text {las }}^{n}$ [54]. Therefore, the collected signal will be proportional to the cube of the laser intensity, assuming that measurement is not made in a saturation regime. A model of an integrated 3pLIF signal is presented in Eq. 2.9 and is an extension of the TALIF signal model:

$$
\mathrm{I}_{\mathrm{LIF}} \propto n \frac{\Omega}{4 \pi} \eta T \sigma_{3 \mathrm{P}}^{(3)}\left(\frac{E}{h \nu}\right)^{3} g a,
$$


where $\mathrm{I}_{\mathrm{LIF}}$ is the integrated LIF signal, $n$ is the state density, $\Omega / 4 \pi$ is solid angle of collection optics, $\eta$ is the quantum efficiency of the detector at the fluorescence wavelength, $T$ is the transmission of the collection optics for the fluorescent wavelength, $\sigma_{3 \mathrm{P}}^{(3)}$ is the three-photon cross section with units of $\mathrm{cm}^{6} s^{2}$ photons ${ }^{-2}, E / h \nu$ is the photon number, $g$ is the gain of the detector, and $a$ is the branching ratio of the excited state. As the number of photon processes increases, the spatial localization of the measurement also increases. Therefore, 3pLIF measurements likely provide the best spatial localization out of the three laser induced methods described in this work.

\subsubsection{3pLIF Transition Specifics}

The same pulsed laser system described above is sufficient to test a 3pLIF Kr scheme. However, the laser mixing crystal (Sec. 4.2.1) is no longer necessary, since the theorized 3pLIF scheme requires $\sim 307 \mathrm{~nm}$ photons. Figure 2.4 shows one possible $3 \mathrm{pLIF}$ scheme that samples the ground state of neutral krypton and is most similar to the TALIF scheme used in this work. Also shown are the relative intensities of each of the decay paths. While there exists an almost identical 3pLIF to that used in TALIF ( $\sim 828 \mathrm{~nm}$ fluorescence), the strongest decay is likely in the visible $(\sim 557 \mathrm{~nm})$.

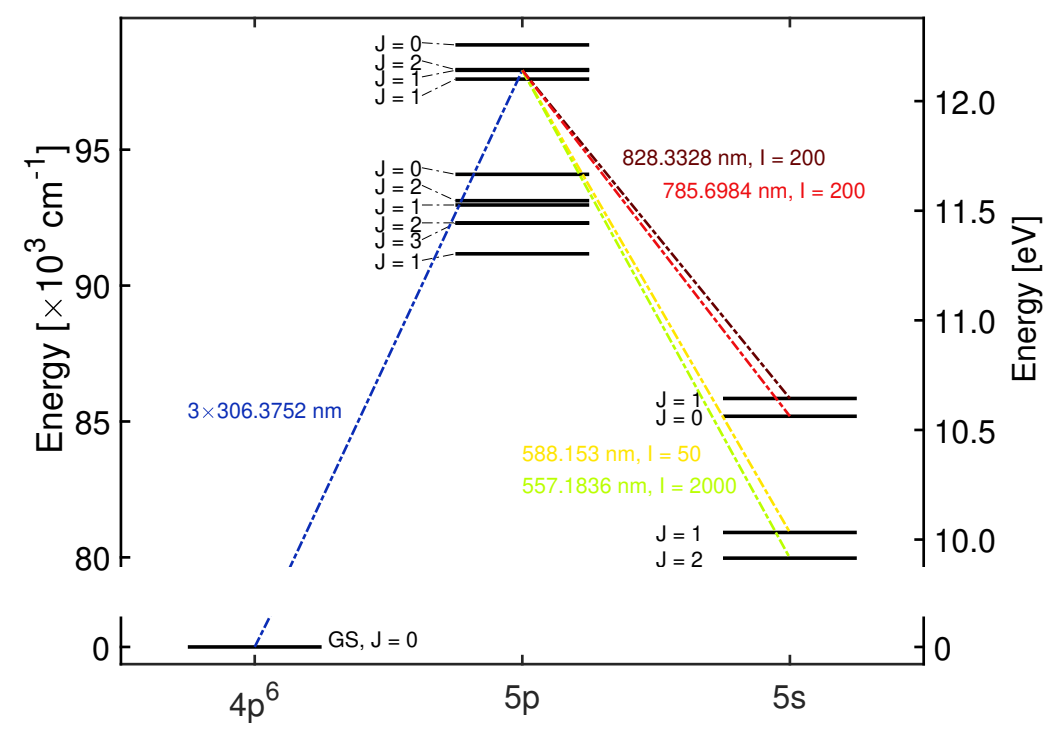

Figure 2.4: Proposed krypton 3pLIF schemes. The ground state of neutral krypton is excited by three $306.3752 \mathrm{~nm}$ photons. The excited state relaxes to one of four states. One decay path is very similar to the TALIF scheme used in this work and occurs at $828.3328 \mathrm{~nm}$. However, the strongest decay occurs at $557.1836 \mathrm{~nm}$. All wavelengths are quoted in vacuum. 
Relevant information for several possible 3pLIF schemes are presented in Tab. 2.2. For quantities that relate directly to a state in the scheme, the subscript of 1,2 , or 3 is used that correspond to the ground, excited, and relaxed state respectively.

\begin{tabular}{cccccccc}
\hline \hline $\begin{array}{c}\text { Transition } \\
\text { Scheme } \\
(\text { Racah })\end{array}$ & $\begin{array}{c}\Delta E_{1-2} \\
(\mathrm{eV})\end{array}$ & $\begin{array}{c}J_{2} \\
(-)\end{array}$ & $\begin{array}{c}3 \mathrm{pLIF} \lambda_{1-2} \\
(\mathrm{~nm})\end{array}$ & $\begin{array}{c}\Delta E_{2-3} \\
(\mathrm{eV})\end{array}$ & $J_{3}$ & $\begin{array}{c}\lambda_{2-3} \\
(-)\end{array}$ & $\begin{array}{c}\text { Intensity } \\
(-)\end{array}$ \\
\hline $4 p^{6} \rightarrow\left(2 \mathrm{P}_{1 / 2}^{\circ}\right) 5 p \rightarrow\left(2 \mathrm{P}_{1 / 2}^{\circ}\right) 5 s$ & 12.100 & 1 & 307.3900 & 1.477 & 1 & 851.1211 & 200 \\
$4 p^{6} \rightarrow\left(2 \mathrm{P}_{1 / 2}^{\circ}\right) 5 p \rightarrow\left(2 \mathrm{P}_{3 / 2}^{\circ}\right) 5 s$ & 12.100 & 1 & 307.3900 & 2.068 & 1 & 599.5510 & 60 \\
$4 p^{6} \rightarrow\left(2 \mathrm{P}_{1 / 2}^{\circ}\right) 5 p \rightarrow\left(2 \mathrm{P}_{1 / 2}^{\circ}\right) 5 s$ & 12.100 & 1 & 307.3900 & 1.538 & 0 & 806.1721 & 100 \\
$4 p^{6} \rightarrow\left(2 \mathrm{P}_{1 / 2}^{\circ}\right) 5 p \rightarrow\left(2 \mathrm{P}_{3 / 2}^{\circ}\right) 5 s$ & 12.100 & 1 & 307.3900 & 2.185 & 2 & 567.4025 & 50 \\
$4 p^{6} \rightarrow\left(2 \mathrm{P}_{1 / 2}^{\circ}\right) 5 p \rightarrow\left(2 \mathrm{P}_{1 / 2}^{\circ}\right) 5 s$ & 12.140 & 1 & 306.3752 & 1.497 & 1 & 828.3328 & 200 \\
$4 p^{6} \rightarrow\left(2 \mathrm{P}_{1 / 2}^{\circ}\right) 5 p \rightarrow\left(2 \mathrm{P}_{3 / 2}^{\circ}\right) 5 s$ & 12.140 & 1 & 306.3752 & 2.108 & 1 & 588.1530 & 50 \\
$4 p^{6} \rightarrow\left(2 \mathrm{P}_{1 / 2}^{\circ}\right) 5 p \rightarrow\left(2 \mathrm{P}_{3 / 2}^{\circ}\right) 5 s$ & 12.140 & 1 & 306.3752 & 2.225 & 2 & 557.1836 & 2000 \\
$4 p^{6} \rightarrow\left(2 \mathrm{P}_{1 / 2}^{\circ}\right) 5 p \rightarrow\left(2 \mathrm{P}_{1 / 2}^{\circ}\right) 5 s$ & 12.140 & 1 & 306.3752 & 1.578 & 0 & 785.6984 & 50 \\
\hline
\end{tabular}

TABLE 2.2: Several possible 3pLIF schemes for neutral krypton. Eight possible schemes are presented, as well as the states involved, the relative energy change between states (in eV), total electronic angular momentum for the excited (2) state and decay (3) state, the 3pLIF pump wavelength $\lambda_{1-2}$, the fluorescence wavelength $\lambda_{2-3}$, and the relative intensity of the fluorescence. Wavelengths are reported in vacuum for all cases. 


\section{Chapter 3}

\section{Broadening Mechanisms}

A measured lineshape is a combination of all of the effects that broaden the line, e.g., natural linewidth, Doppler, Zeeman, etc. [55] Plasma quantities in this work are crucially dependent on the Doppler broadening of the velocity distribution and accurate consideration of all dominant broadening mechanisms is critical for reliable results. In the following sections, important mechanisms of line broadening are discussed. All simulated plots related to TALIF measurements are expressed terms of the fundamental absorption transition $\left(\lambda_{\circ} \sim 100 \mathrm{~nm}\right)$.

\subsection{Natural Linewidth}

Natural linewidth is the result of the uncertainty principle. This is the absolute minimum resolvable lineshape and is typically negligible compared to other broadening effects. This broadening effect is proportional to the sum of all the spontaneous emission coefficients from the upper and lower states of the probe transition. The natural linewidth for the deuterium transition used in this work is $\sim 60 \mathrm{MHz}$, which is two orders of magnitude less than the absorbed laser linewidth and three orders of magnitude less than the expected effect of Doppler broadening. Neglecting this effect introduces $\ll 0.1 \%$ error to the measured neutral deuterium temperature and therefore the natural linewidth is neglected for TALIF analysis.

The spontaneous coefficients for the probed states are not available for the iodine scheme investigated here. As a consequence, the natural linewidth of the transition is not calculated. Even so, the Doppler linewidth for transitions in the visible and UV spectrum is normally about 
two orders of magnitude or more larger than the natural linewidth and therefore the natural linewidth is also neglected for the iodine transition probed here [55, p. 85].

\subsection{Pressure/Stark Broadening}

Stark broadening arises from two separate effects. The first is analogous to Zeeman broadening. In this case, an external electric field splits a transition into several transitions that have slight shifts in energy. If the energy shifts are modest, the measured lineshape is a superposition of all split states. For the work presented here, there are no external electric fields and therefore, this specific type of Stark broadening is not a broadening factor. Second, when a gas or plasma pressure (density) increases, particles are forced closer to each other and microelectric fields created by charged particles cause an internal Stark broadening effect. Galante estimated that the Stark broadening for hydrogen in a plasma similar to the plasma investigated in this work is $\sim 10 \mathrm{MHz}$ and therefore this effect is also neglected for the TALIF measurements [30]. Similarly, this effect is not expected to be a dominating factor for the iodine measurements presented here since the plasma is expected to be weakly ionized.

\subsection{Laser Linewidth}

The linewidth of a pulsed laser is inversely proportional to the pulse length and, as a result, a pulsed laser (similar to the one used in this work) can have a linewidth that is comparable to the measured absorption linewidth. The measured absorption linewidth is a convolution of the Doppler profile and the laser linewidth. ${ }^{1}$ The specifications for the TALIF laser are quoted at the dye conversion stage [71]. However, additional effects modify the laser linewidth at the transition wavelength, namely the frequency conversion processes (Sec. 4.2.1). A key point to consider is that two photons are being absorbed simultaneously, resulting in a convolved absorption laser lineshape of two single-photon profiles as seen by the absorbing species:

$$
f_{\mathrm{TP}}=f_{\mathrm{SP}} * f_{\mathrm{SP}},
$$

where $f_{\mathrm{TP}}$ is the two-photon lineshape and $f_{\mathrm{SP}}$ is the single-photon lineshape. As discussed in Sec. 4.2.2, the lineshape of a photon produced by the TALIF laser system assumes a Lorentzian

\footnotetext{
${ }^{1}$ This is only true if the Doppler broadening is the single dominating mechanism.
} 
profile (Eq. 4.3). In reality, Eq. 4.3 should be normalized such that the integral over all time and frequency returns the energy per pulse of the system. However, the amplitude of the lineshape does not contribute to this discussion and is only mentioned here for completeness.

The convolution of two single-photon lineshapes (one for each partial excitation involved in the TALIF process) yields a convolved linewidth that is a factor of two wider than the singlephoton linewidth. The details of the convolution of two Lorentzian functions are presented in App. A. Combining the increased linewidth with the frequency conversion-induced linewidth increase, the laser lineshape increases by a total factor of 6 from the dye conversion stage to the absorption wavelength:

$$
\Gamma_{102}=6 \Gamma_{615}
$$

where $\Gamma_{102}$ is the HWHM of the absorbed laser linewidth and $\Gamma_{615}$ is the HWHM of the laser linewidth specified at the dye stage. In an effort to mitigate confusing language, post-dye conversion wavelengths are referred to as dye frame, i.e., as the wavemeter reports, and the "100 nm" space wavelengths are referred to as the absorber frame, i.e., what the electrons in the neutral atom see. The absorber encounters a photon with an effective linewidth of $\sim 5.7$ $\mathrm{GHz}(0.2 \mathrm{pm})$. A measured TALIF lineshape will manifest itself as a convolution of a Gaussian lineshape (Doppler component) and a Lorentzian component (laser contribution):

$$
V(\nu)=\int_{-\infty}^{\infty} L(x) G(\nu-x) d x
$$

where $V(\nu)$ is the Voigt lineshape function, $L$ is the Lorentzian lineshape function, and $G$ is the Gaussian lineshape function [72]. The functional form presented above is not very useful when fitting the Voigt function to experimental data. In this case, the Voigt profile is written as:

$$
\begin{aligned}
V(\nu ; \gamma, \Gamma) & =\operatorname{Re}(w[z]) \\
& =\operatorname{Re}\left(w\left[\frac{\nu+i \Gamma}{\sqrt{2} \gamma}\right]\right),
\end{aligned}
$$


where $\gamma$ is the Gaussian width, $\Gamma$ is the Lorentzian width, and $w(z)$ is the Faddeeva function, defined as

$$
\begin{aligned}
w(z) & =\exp \left(-z^{2}\right) \operatorname{erfc}(-i z) \\
& =\operatorname{erfcx}(-i z) \\
& =\exp \left(-z^{2}\right)\left(1+\frac{2 i}{\pi} \int_{0}^{z} \exp \left(t^{2}\right) d t\right)
\end{aligned}
$$

and erfc is the error function. Abrarov et al. provide a Matlab ${ }^{\circledR}$ script that estimates the Faddeeva function within an accuracy of $10^{-13}$ and thus allows for the Gaussian width to be left as a fit parameter which is extracted directly from a fit of Eq. 3.4 to the measured lineshape to determine the Gaussian width [73].

Figure 3.1 shows a simulated measured lineshape (dark blue) that is composed from the convolution of a deuterium NVDF with a corresponding temperature of $0.2 \mathrm{eV}$ (light blue) and the expected laser lineshape (gray). While the laser linewidth is small, ignoring the laser linewidth effects introduce $\mathrm{a} \sim 10 \%$ error in deuterium temperature.

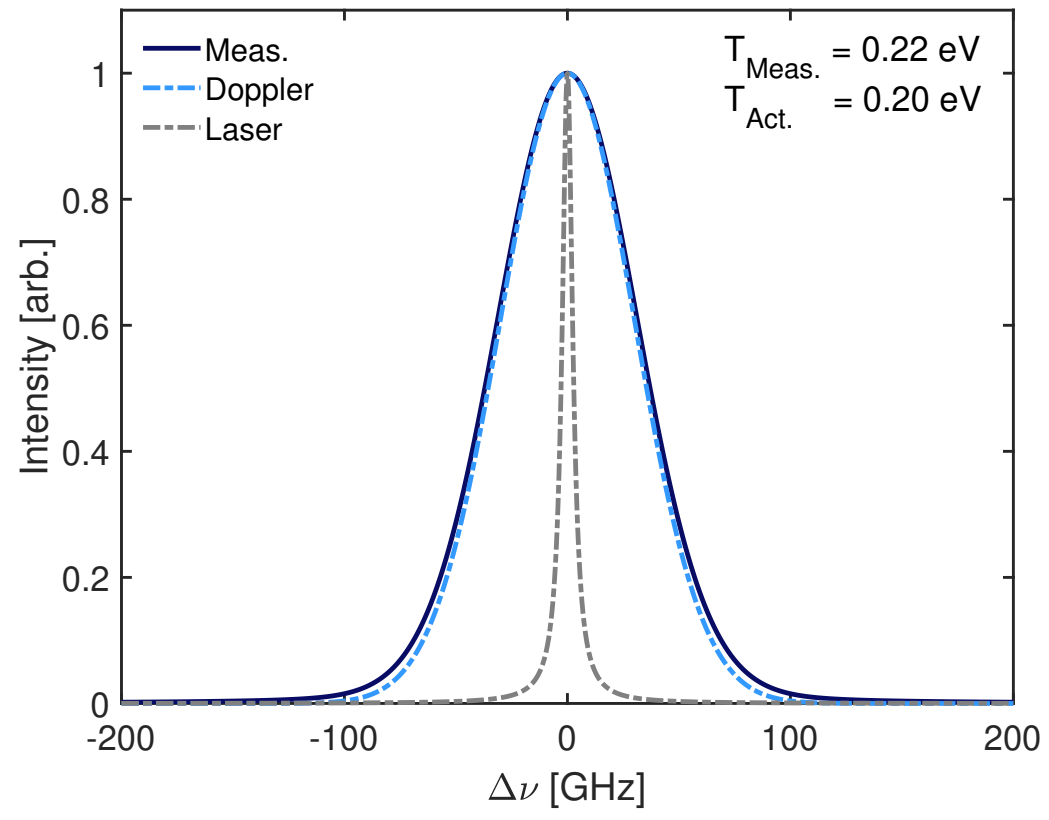

Figure 3.1: Simulated measured lineshape (dark blue), NVDF for a temperature of $0.2 \mathrm{eV}$ for deuterium (light blue), and laser lineshape (gray). The laser lineshape has a FWHM of $\sim 5.70 \mathrm{GHz}$ and the NVDF has a FWHM of $\sim 71 \mathrm{GHz}$. The convolved simulated measured profile slightly broadened to $\sim 74 \mathrm{GHz}$.

It is expected that neutral deuterium atoms will be born with shared dissociation energy of 
$>1 \mathrm{eV}$, as described by averaging over the Franck-Condon region ${ }^{2}$ [74], though Galante et al. found that the neutral deuterium temperatures in a helicon source are typically much lower. The suspected reason is that while neutrals may be born as Franck-Condon neutrals $(T \sim 2 \mathrm{eV})$, there is no heating mechanism present for the atomic neutrals and therefore the temperature tends to relax towards chamber wall temperature, i.e., $T \ll 2 \mathrm{eV}$ [58]. For distributions exhibiting temperatures in the tenths of eVs or greater, laser linewidth effects contribute marginally to the measured deuterium NVDF linewidth $(\sim 10 \%)$. However, if a stronger cooling mechanism were present and deuterium temperatures were closer to room temperature, or a heavier species were to be investigated (see Fig. 3.2), the laser linewidth introduces significant effects to the measured lineshape.

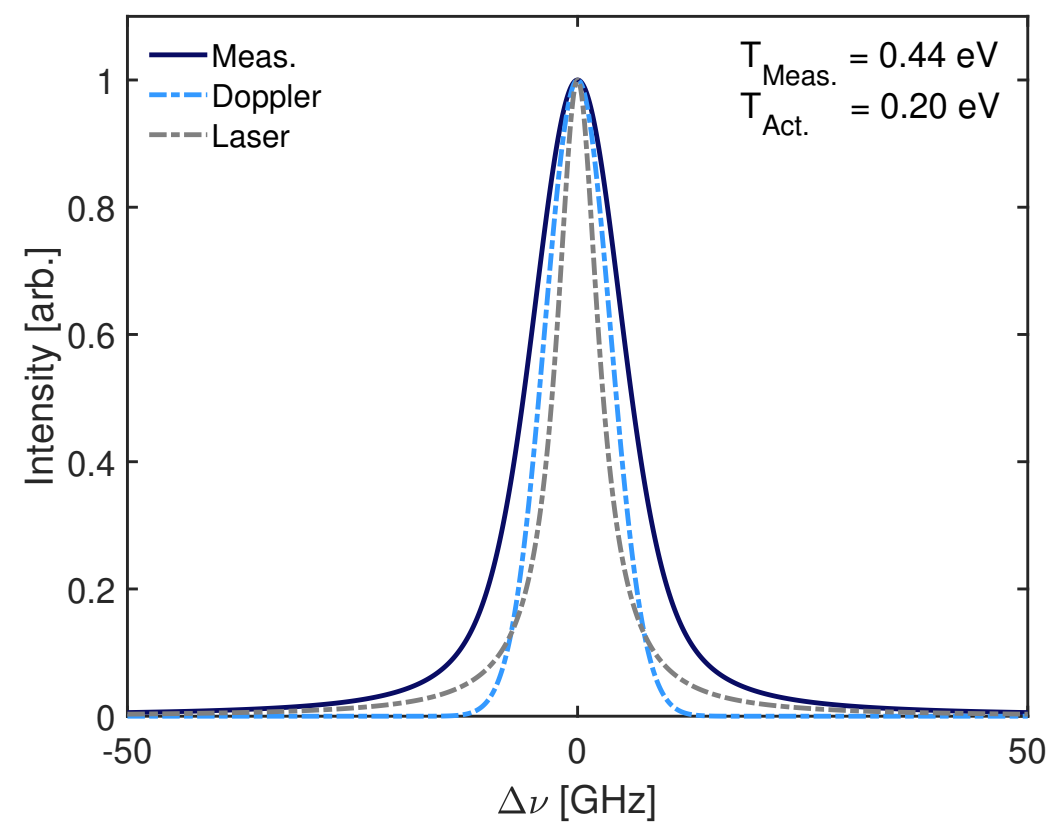

FiguRE 3.2: Simulated neutral distribution for a temperature of $0.2 \mathrm{eV}$ for xenon (light blue). The laser lineshape is given by the gray Gaussian and has a FWHM of $\sim 5.7 \mathrm{GHz}$. The NVDF has a FWHM of $\sim 9 \mathrm{GHz}$. The convolved simulated measured profile is given by the dark blue line and is broadened to $\sim 12 \mathrm{GHz}$.

Figure 3.3 shows the percent difference between measured temperature and actual temperature obtained from deconvolving a broad laser profile for a range of distribution temperatures from room temperature $(0.026 \mathrm{eV})$ up to hot deuterium neutrals $(1 \mathrm{eV})$.

\footnotetext{
${ }^{2}$ The Frank-Condon region is the range of inter-nuclear separation that is associated with the lowest vibrational state. That is, an incident electron can interact with the particle anywhere over this range. As such, the region includes multiple possible transitions for different incident electron energies.
} 


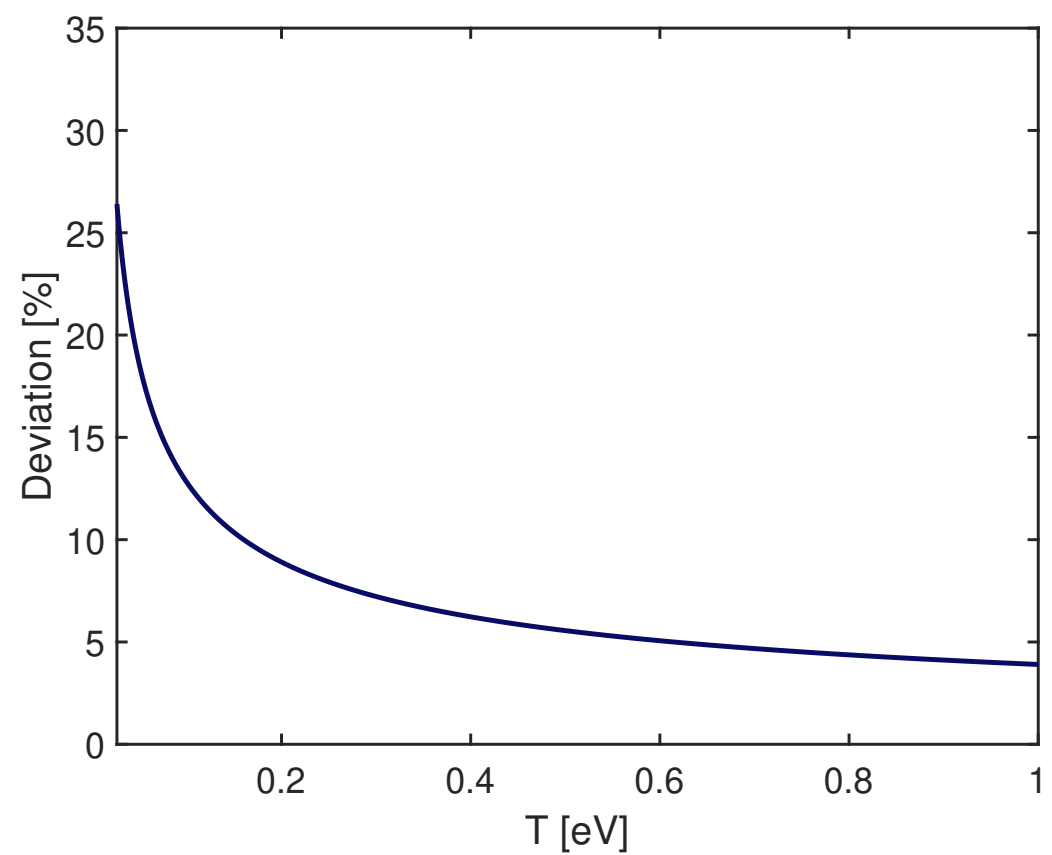

Figure 3.3: Percent deviation produced by the laser linewidth for a given NVDF temperature. Difference between measured temperature and actual population temperature can be as high as $\sim 35 \%$ for temperatures near room temperature, but approach values smaller than $5 \%$ for hot neutrals $(\sim 1 \mathrm{eV})$.

At low temperatures, ignoring the laser linewidth introduces errors as large as $\sim 35 \%$ for deuterium. For the temperatures quoted in this work $(\sim 0.2 \mathrm{eV})$ the errors introduced by neglecting the laser linewidth correction are $\sim 10 \%$.

\subsection{Zeeman Splitting}

Zeeman splitting is the splitting of energy levels induced by an external magnetic field [75]. To understand the Zeeman effect and how the strength of the magnetic field changes electronic transitions, intrinsic magnetic effects-namely fine and hyperfine splitting-are first discussed.

Electron interaction with induced fields, nuclei, and external fields modify the energy levels of the unperturbed transition. The first of these effects to be discussed arises from the motion of an electron around the nucleus. In the most simple case, the motion of the electron produces a current which sets up an internal magnetic field $B_{\text {int }}$. This magnetic field exerts a torque on the electron's intrinsic angular momentum (spin). It is counter-intuitive to think about an electron producing a field that, in turn, disrupts its own properties. To visualize this, it is easier to move to the frame of the electron. The electron does not interact with a self-produced field. Instead, the magnetic field is created by the positively charged nucleus orbiting it. It is the motion of the 
nucleus in the frame of the electron that produces the magnetic field. Some authors choose to describe the creation of the magnetic field as an interpretation of a static electric field produced by the nucleus in the frame of the moving electron. This type of splitting is known as spin-orbit coupling, which is a component of fine-structure splitting. Only the spin-orbit contribution to the fine structure is considered in this work.

The energy shift of a spin-orbit split transition can be determined by adding a small perturbation to the Hamiltonian $\mathcal{H}^{(0)}$ of the system. The interaction of the internal magnetic field and the electron spin is described by

$$
\Delta E=-\vec{\mu}_{\mathrm{S}} \cdot \vec{B}_{\text {int }}
$$

where $\vec{\mu}_{\mathrm{S}}$ is the spin magnetic moment. The magnetic moment is given by

$$
\vec{\mu}_{\mathrm{S}}=-\frac{g_{\mathrm{S}} \mu_{\mathrm{B}} \vec{S}}{\hbar}
$$

where $g_{\mathrm{S}}$ is the Landé g-factor for the electron spin, $\mu_{\mathrm{B}}$ is the Bohr magneton, and $\hbar$ is the reduced Planck's constant. The magnetic field created by the nuclear charges can be written as (in the simple Bohr orbit model)

$$
\vec{B}_{\text {int }}=\frac{e}{4 \pi \epsilon_{\circ} c^{2}} \frac{1}{m_{\mathrm{e}} a_{\circ}^{3}} \vec{L}
$$

where $e$ is the fundamental charge, $\epsilon_{\mathrm{o}}$ is the permittivity of free space, $c$ is the speed of light, $m_{\mathrm{e}}$ is the mass of an electron, $a_{\circ}$ is Bohr's radius, and $\vec{L}$ is the orbital angular momentum of the electron. Combining the above equations, the perturbation to the unperturbed Hamiltonian is given by

$$
\mathcal{H}_{\mathrm{SO}}=\frac{g_{\mathrm{S}} \mu_{\mathrm{B}} e}{4 \pi \epsilon_{\circ} c^{2} m_{\mathrm{e}} a_{\mathrm{o}}^{3} \hbar} \vec{L} \cdot \vec{S}
$$

Spin-orbit coupling is an intrinsic effect that cannot be "turned off" and will still be present in the absence of all external fields. Spin-orbit coupling is responsible for states splitting into $J=|L-S|$ to $|L+S|$ levels. When working with the spin-orbit Hamiltonian it is important to mention that a coupled basis set is required to diagonalize this perturbation.

Before diving into the Zeeman effect, a finer splitting that is analogous to the fine-structure splitting is briefly discussed here and is outlined in more detail in Sec. 3.5. This type of 
splitting is a result of the spin of the nucleus $\vec{I}$ interacting with the total electronic angular momentum and is orders of magnitude smaller than the spin-orbit splitting [76]. This type of splitting is known as hyperfine splitting. Hyperfine splitting is not expected to be a significant line broadening mechanism for deuterium, but is significant for I II. Since the fluorescence schemes exploit an energy level with a specific $J$, fine-structure splitting is not a source of line broadening. Nevertheless, to be able to interpret the physics of Zeeman splitting, knowledge of the fine-structure splitting is critical.

Charged particles interact with an external magnetic field in the same manner that they interact with the internal magnetic field (Eq. 3.6), and this interaction is known as the Zeeman effect [75]. The Zeeman effect is described by three unique regimes based on the relative strength of the magnetic field: if the external magnetic field is very weak, then the Zeeman effect is said to be in the weak-field limit; if the external magnetic field is very strong, the Zeeman effect is said to be in the strong-field limit, also known as the Paschen-Back effect [77]. If the external magnetic field is neither very strong nor very weak, then the Zeeman effect is in the intermediate field limit. Most authors use the internal magnetic field as a reference quantity to describe the relative strength of the external magnetic field $[78,79]$. Here, a list of magnetic field thresholds for each of these regimes as well as the differences in the Zeeman effect in these regimes is given. The values presented in this work are unique to the specific transition in deuterium and a separate analysis should be carried out for other species and transitions.

The Hamiltonian addition for the Zeeman effect is

$$
\begin{aligned}
\mathcal{H}_{\mathrm{Z}} & =-\vec{\mu}_{L} \cdot \vec{B}_{\text {ext }}-\vec{\mu}_{S} \cdot \vec{B}_{\text {ext }} \\
& =\frac{g_{\mathrm{L}} \mu_{\mathrm{B}} \vec{L}}{\hbar} \cdot \vec{B}_{\text {ext }}+\frac{g_{\mathrm{S}} \mu_{\mathrm{B}} \vec{S}}{\hbar} \cdot \vec{B}_{\text {ext }},
\end{aligned}
$$

where $B_{\text {ext }}$ is the external magnetic field, $g_{L}$ and $g_{S}$ are the Landé g-factors for the orbital and spin angular momenta, respectively, with respective values of 1 and 2.0023192 (many times taken to be simply 2). The other constants are the same as listed in Eq. 3.7.

To estimate the thresholds for each regime using the method described in Ref. [79], the entire Hamiltonian of the system must first be considered. In general, the system is well described by the Hamiltonian

$$
\mathcal{H}_{\text {total }}=\mathcal{H}^{(0)}+\mathcal{H}_{\mathrm{SO}}+\mathcal{H}_{\mathrm{Z}}
$$


In the weak-field limit, the Zeeman effect is much smaller than the spin-orbit coupling. As a result, $\mathcal{H}^{(0)}$ and $\mathcal{H}_{\mathrm{SO}}$ are grouped as an effective unperturbed Hamiltonian $\check{\mathcal{H}}^{(0)}$. The Zeeman effect is now treated as a small perturbation. Since the spin-orbit Hamiltonian is included as part of the unperturbed Hamiltonian and the unperturbed Hamiltonian was described by a coupled basis set, the Zeeman effect in the weak-field limit is also expressed in the same coupled basis set. Therefore the Zeeman effect when $B_{\text {ext }} \ll B_{\text {int }}$ causes an energy shift that is unique to the coupled magnetic quantum number $m_{J}$.

However, in the strong-field limit, the unperturbed Hamiltonian and the Zeeman perturbation are grouped together, while the spin-orbit effect is treated as the perturbation Hamiltonian. In this case, it is easier to see the "broken" coupling between the orbital momentum and spin momentum. Since the unperturbed Hamiltonian commutes with all angular momenta, the uncoupled basis describes the system (i.e., $\left|n l m_{L} m_{s}\right\rangle$ ). The spin-orbit contribution is then expressed in the uncoupled basis. In this case, the threshold is defined as $B_{\text {ext }} \gg B_{\text {int }}$.

From Eq. 3.8, the internal magnetic field for deuterium is expected to be $\sim 10 \mathrm{~T}$ [79]. (see Prob. 6.18). However, other authors suggest that the strong-field limit occurs at much lower magnetic fields [76]. In this work, the Paschen-Back regime is invoked when the Zeeman splitting is much larger than the spin-orbit splitting, e.g., an order of magnitude or larger. For the transition probed in this work, the spin-orbit splitting is $\sim 1 \mathrm{GHz}$. The strong-field limit is then defined as the magnetic field that produces a Zeeman-split transition that is on the order of $10 \mathrm{GHz}$. The weak-field limit is defined as a magnetic field that produces splitting on the order of 0.1 GHz. Fields that cause splitting between these values are treated in the intermediate field limit.

Table 3.1 lists the strong and weak-field estimates for the ground state and excited state of deuterium for this work for both methods of determining the field thresholds. Here $\vec{B}_{\text {int }}$ is defined as the internal field described by Eq. 3.8. $\vec{B}_{\text {ext }, 1 w}$ is the weak-field limit based on the magnitude of the external magnetic field compared to $\vec{B}_{\text {int }}$ and $\vec{B}_{\text {ext, } 1 s}$ is similarly defined. $\vec{B}_{\text {ext }, 2 w}$ is the weak-field limit based on the magnitudes of the resultant line splitting and $\vec{B}_{\text {ext }, 2 s}$ is the strong-field limit for the same comparison.

In the weak-field limit, each unperturbed energy level splits into separate levels determined by

$$
\Delta \nu=\Delta E / h=\mu_{\mathrm{B}} g_{J} m_{J} B_{\mathrm{ext}},
$$




\begin{tabular}{lccccc}
\hline \hline $\begin{array}{l}\text { Transition } \\
(-)\end{array}$ & $\begin{array}{c}\vec{B}_{\text {int }} \\
(\mathrm{T})\end{array}$ & $\begin{array}{c}\vec{B}_{\text {ext,1w }} \\
(\mathrm{T})\end{array}$ & $\begin{array}{c}\vec{B}_{\text {ext,1s }} \\
(\mathrm{T})\end{array}$ & $\begin{array}{c}\vec{B}_{\text {ext }, 2 w} \\
(\mathrm{~T})\end{array}$ & $\begin{array}{c}\vec{B}_{\text {ext,2s }} \\
(\mathrm{T})\end{array}$ \\
\hline $1^{2} S_{1 / 2} \rightarrow 3^{2} D_{5 / 2}$ & $\sim 30$ & $\lesssim 1$ & $\gtrsim 100$ & $\lesssim 0.003$ & $\gtrsim 0.3$ \\
\hline
\end{tabular}

TABLE 3.1: Magnetic field thresholds for weak-field limit and strong-field limit for the two methods described in the text. $\vec{B}_{\text {int }}$ is the value of the internal magnetic field produced by the electron motion around the proton, $\vec{B}_{\text {ext }, 1 w}$ is the weak-field limit for comparing external field to internal field, $\vec{B}_{\text {ext }, 1 s}$ is the strong-field limit for the same comparison. $\vec{B}_{\text {ext }, 2 w}$ is the weak-field limit for comparing energy splitting of spin-orbit effects and Zeeman effects, and $\vec{B}_{\text {ext } 2 s}$ is the strong-field limit for the same comparison. The second approach is adopted as the limits for the strong- and weak-field cases for this work.

where $h$ is Planck's constant, $\mu_{\mathrm{B}}$ is the Bohr magneton, $g_{J}$ is the combined Landé $\mathrm{g}$ factor for the coupled basis, $m_{J}$ is the total angular momentum magnetic quantum number, and $\Delta \nu$ is the shift of the energy level in frequency space. The Landé g-factor for the coupled angular momentum is given by

$$
g_{J}=1+\frac{J(J+1)-L(L+1)+S(S+1)}{2 J(J+1)},
$$

where $J$ is the total electronic angular momentum, $L$ is the orbital angular momentum, and $S$ is the spin (in this case always $1 / 2$ ). In the strong-field limit, each unperturbed energy level splits into separate levels determined by

$$
\Delta \nu=\Delta E / h=\mu_{\mathrm{B}} B_{\mathrm{ext}}\left(m_{L}+2 m_{S}\right) .
$$

In the presence of an external magnetic field the ground state of deuterium splits into 2 states. The upper state of the TALIF transition splits into 6 states in the weak-field limit and 7 states in the strong-field limit. Not all transitions between the split states are allowed as the selection rules for a two-photon absorption transitions are $\Delta m=0$ ( $\pi$-transitions) or $\Delta m= \pm 2$ ( $\sigma$-transitions) since the electron is absorbing two quanta of momentum ${ }^{3}[30,80]$. Figures 3.4 and 3.5 show the level splitting and allowed transitions for the probed states for the weak and strong-field cases, respectively.

\footnotetext{
${ }^{3}$ It is a general case that the selection rules are $\Delta m=0$ ( $\pi$-transitions) or $\Delta m= \pm 2(\sigma-$ transitions $)$ but in some cases, even these transitions are forbidden. The reader is directed to Ref. [80] for more details.
} 


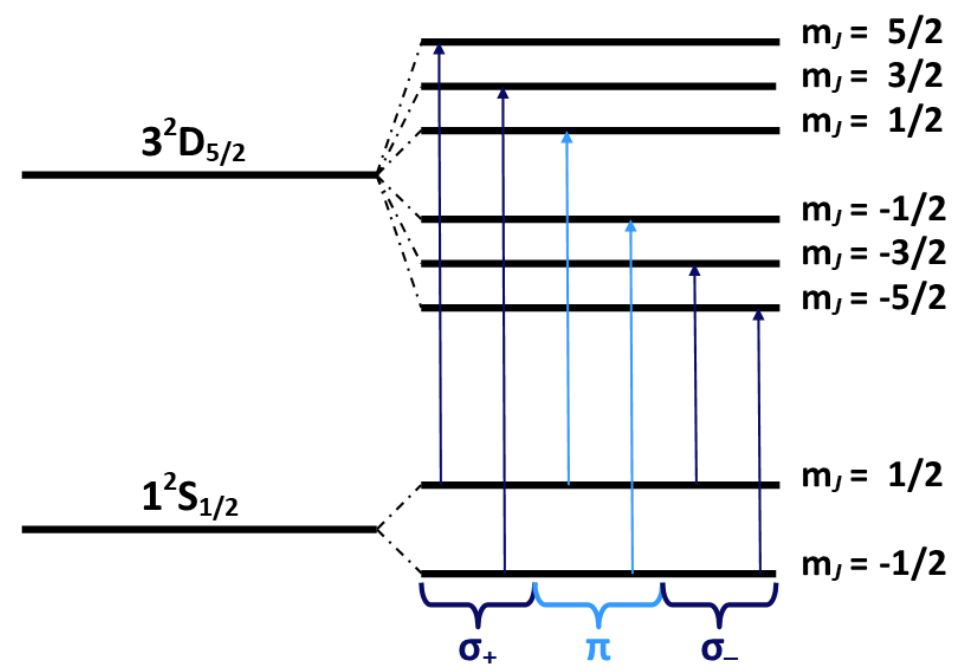

Figure 3.4: Allowed Zeeman transitions in the weak-field limit. The selection rules are $m_{J}=0$ ( $\pi$-transitions) and $m_{J}= \pm 2$ ( $\sigma-$ transitions). The energy levels are split into unique levels based on $m_{J}$.

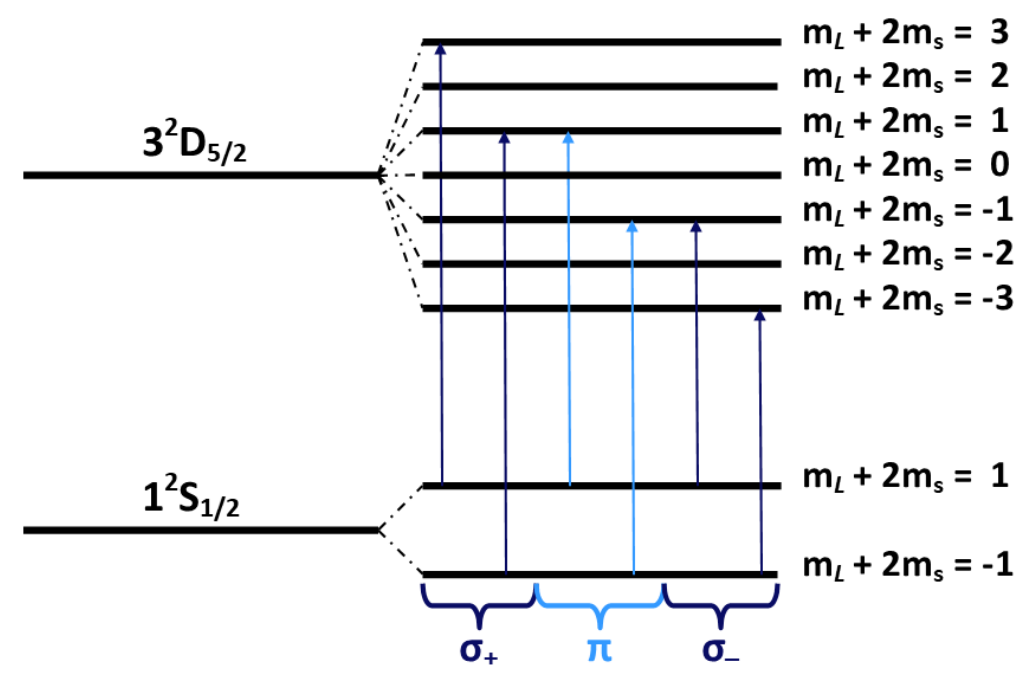

Figure 3.5: Allowed Zeeman transitions in the strong-field limit. The selection rules are $m_{L}+2 m_{S}=0$ ( $\pi$-transitions $)$ and $m_{L}+2 m_{S}= \pm 2(\sigma-$ transitions $)$. The energy levels are split into levels based off the uncoupled combinations of $m_{L}$ and $m_{S}$.

The external magnetic field produced at the location of the TALIF measurements in ProtoMPEX is nominally $\sim 0.3 \mathrm{~T}$. At these fields, the transitions are well described by the PaschenBack regime and the frequency shift of each energy level shift is calculated from Eq. 3.14. Selection rules determine which transitions between the split energy levels are allowed. Prediction of relative amplitudes for each Zeeman-split transition is well understood for single-photon LIF $[81,82]$. Relative amplitudes of each single-photon transition are calculated using quantum 
numbers and the Landé factors for the upper and lower states [81]. However, accurate prediction of two-photon Zeeman-split transitions is much more complex and tedious to calculate [83]. Fortunately, for the TALIF measurements reported here, the Zeeman structure does not add a significant source of broadening. To confirm that this effect can be neglected, a simulated deuterium NVDF corresponding to $0.2 \mathrm{eV}$ was fit 1000 times to a three-Gaussian function that represent the Zeeman-split spectrum. The locations of each transition are constrained to obey the calculated Zeeman-splitting and each transition is constrained to have the same thermal temperature. However, the relative amplitudes are left as free parameters in the fitting routine with two constraints: the $\sigma$-clusters have the same amplitude and all amplitudes do not exceed the observed NVDF intensity. Figure 3.6 shows one possible strong-field regime Zeeman-split lineshape for two-photon transitions in deuterium.

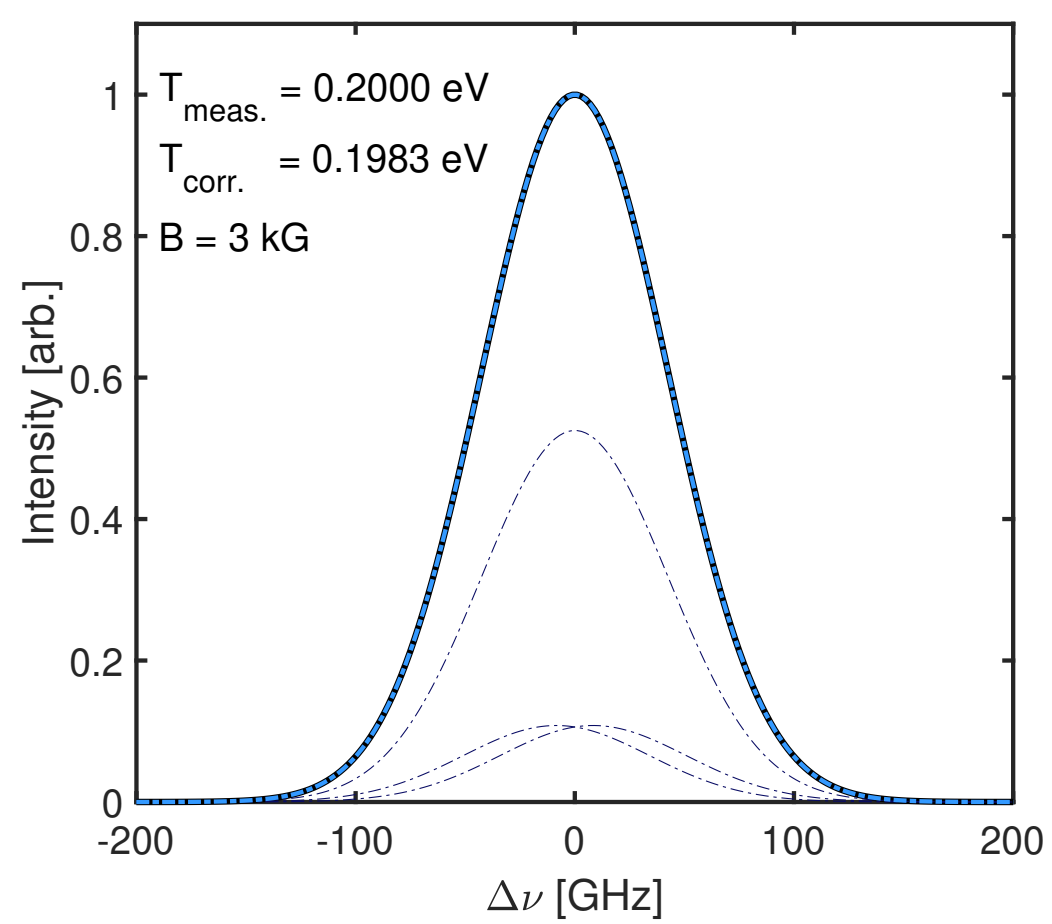

FiguRE 3.6: Estimated strong-field transitions shift for a external magnetic field of $0.3 \mathrm{~T}$ for a simulated distribution function corresponding to $0.2 \mathrm{eV}$. The simulated measured Zeeman-split lineshape is indicated by the solid dark blue line and individual Zeeman transitions are indicated by the light blue dashed lines. The broadening effect at this magnetic field is small.

It was found that Zeeman-induced broadening introduces a negligible increase to the temperature (at worst-4\%). If left uncorrected, it is estimated from the Monte Carlo fitting method that an average uncertainty of $2.50 \% \pm 0.01 \%$ is introduced into the measured temperature, a negligible effect compared to other sources of uncertainty from broadening effects. While there 
are technically 6 transitions shown in Fig. 3.5, the $\sigma$-transitions and $\pi$-transitions are degenerate, i.e., $\sigma_{+}$produces a single peak, $\sigma_{-}$produces a single peak, and $\pi$ produces a single peak to make a total of three unique transitions. This is seen from Eq. 3.14 since the difference from upper to lower states have the same energy differences for the sets of $\sigma$ and $\pi$ transitions. In Eq. 3.12 , the value of $g_{J}$ is different for the upper and lower state producing 6 unique transitions.

\subsection{Hyperfine Splitting}

The iodine lineshape measured in this work is exceptionally broad due to the hyperfine structure of the probed transition. Since LIF probes a specific combination of pump and fluorescence transitions, spin-orbit coupling does not cause significant broadening of the measured lineshape. However, hyperfine transitions for a given transition all have the same $\Delta J$; all hyperfine transitions during a single-photon LIF measurement are excited and each has a unique fluorescence wavelength. The iodine hyperfine splitting arises solely from the interactions of electron momenta and the nuclear spin. There is a second phenomena that is often categorized as hyperfine splitting which arises from multiple isotopes in the target sample. Here, isotopic splitting is considered separately in Sec. 3.6. Additionally, iodine has one naturally occurring isotope and therefore there is no isotopic-splitting effect.

The physical mechanism responsible for hyperfine splitting is the addition of the nuclear spin $I$ and the total electronic angular momentum $J$. The addition yields a unique total atomic angular momentum value $F=I+J$ that is analogous to $J$ when calculating the energy levels produced from fine-structure splitting. $F$ takes on values from $|I-J|$ to $I+J$ in steps of $I$. The Hamiltonian for hyperfine splitting is a combination of magnetic dipole and electric quadrupole interactions. Each contribution causes a shift in energy from the unsplit state. The Hamiltonian for the magnetic dipole moment is

$$
\hat{\mathcal{H}}_{\mathrm{M}}=A \hat{I} \cdot \hat{J}
$$

where $A$ is an experimentally determined coupling coefficient. The energy shift $E_{\mathrm{M}}$ is the eigenvalue of the Hamiltonian, $\hat{\mathcal{H}}_{\mathrm{M}} \psi=E_{\mathrm{M}} \psi$. Defining

$$
\begin{aligned}
F^{2} & =(I+J)^{2} \\
& =I^{2}+2 I \cdot J+J^{2},
\end{aligned}
$$


the energy shift arising from the magnetic dipole contribution is

$$
\Delta E_{\mathrm{M}}=\frac{A \hbar}{2}(F(F+1)-I(I+1)-J(J+1)) .
$$

The Hamiltonian describing the hyperfine interaction induced by the electric quadrupole moment is quite complex. For an in-depth explanation of the Hamiltonian for the electric quadrupole interaction, the reader is directed to Refs. [72], [76], and [84]. Here the energy shift associated with the electric quadrupole hyperfine interaction is simply provided:

$$
\Delta \nu_{\mathrm{Q}}=\frac{B}{4} \frac{(3 / 2) C(C+1)-2 I(I+1) J(J+1)}{I(2 I-1) J(2 J-1)},
$$

where $\Delta \nu_{\mathrm{Q}}$ is the shift in energy from the un-split state caused by the electric quadrupole hyperfine interaction, $B$ is an experimentally determined coupling coefficient, and

$$
C=F(F+1)-I(I+1)-J(J+1) .
$$

Each hyperfine split state is shifted by an energy of

$$
\Delta \nu=\frac{A}{2} C+\frac{B}{4} \frac{(3 / 2) C(C+1)-2 I(I+1) J(J+1)}{I(2 I-1) J(2 J-1)} .
$$

Selection rules dictate the allowed transitions between the hyperfine split states. Analogously to the selection rules that govern ordinary transitions, the allowed transitions for hyperfine transitions, fifteen in total, are restricted to those where the total atomic angular momentum $F$ changes by 0 or \pm 1 . Hargus et al. calculated the relative intensity of each allowed transition from Russell-Saunders coupling [22]. The rules that govern the relative intensities are shown in Table 3.2. Figure 3.7 shows the hyperfine levels and allowed transitions for the iodine transition probed in this work. Beneath each transition is the theorized relative intensity.

\subsection{Isotopic Splitting}

When isotopes are present, a measured lineshape is sometimes broadened since there are slight differences in energy of the transitions of the isotopes. Two mechanisms are responsible for the energy shift between isotopes [84]. The first mechanism consists of two terms, the normal 


\begin{tabular}{cccc}
\hline \hline & $F-1 \rightarrow F$ & $F \rightarrow F$ & $F+1 \rightarrow F$ \\
\hline$\Delta J= \pm 1$ & $S=\kappa \frac{(Q+I+1)(Q+I)(Q-I)(Q-I-1)}{F}$ & $S=\kappa \frac{(Q+I+1)(Q-I)(W+I)(W-I-1)(2 F+1)}{F(F+1)}$ & $S=\kappa \frac{(W+I)(W+I-1)(W-I-1)(W-I-2)}{F+1}$ \\
$\Delta J=0$ & $S=\kappa \frac{(Q+I+1)(Q-I)(W+I+1)(W-I)}{F}$ & $S=\kappa \frac{[J(J+1)+F(F+1)-S(S+1)]^{2}(2 J+1)}{F(F+1)}$ & $S=\kappa \frac{(Q+I+2)(Q-I+1)(W+I)(W-I-1)}{F+1}$ \\
\hline \hline
\end{tabular}

TABLE 3.2: Equations that govern the relative intensity of hyperfine transitions [78]. $\kappa$ is an arbitrary constant common to all states, $Q=F+J$, and $W=F-J[22]$.

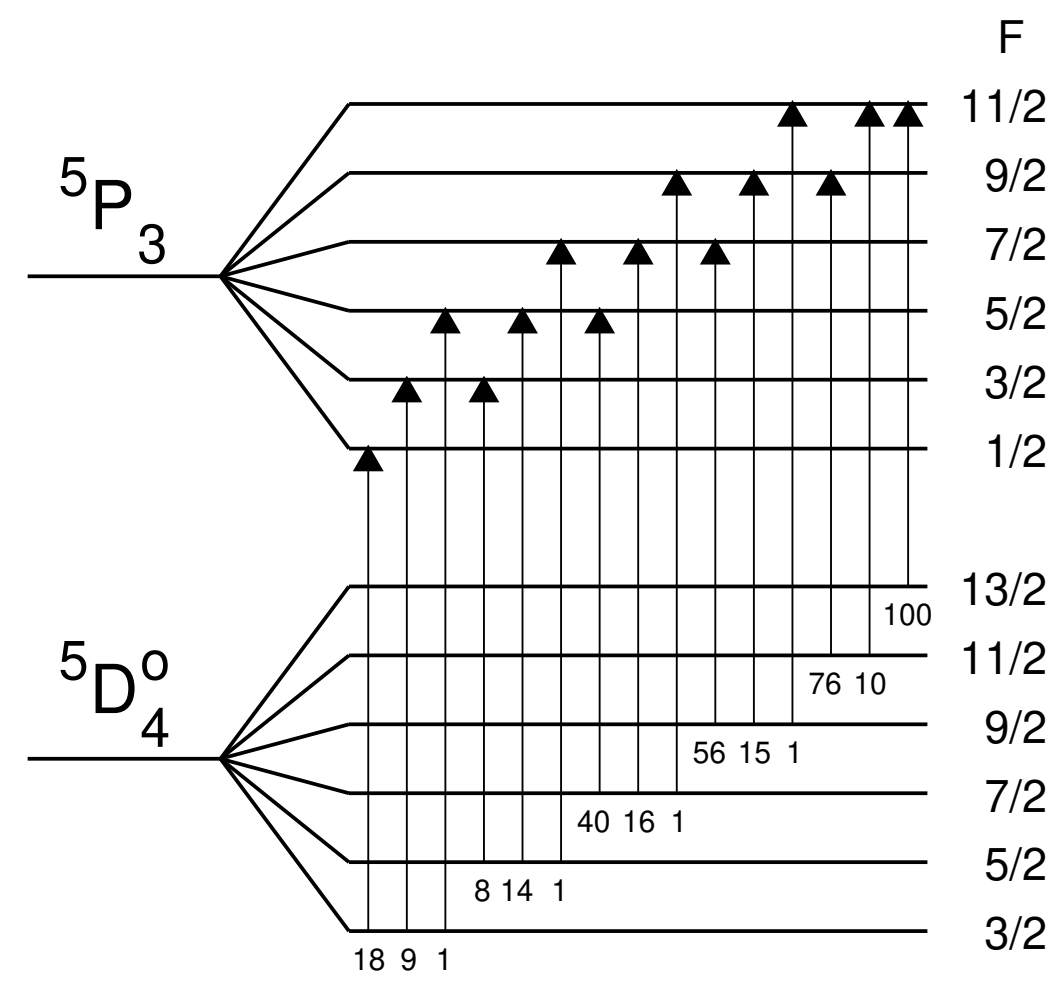

Figure 3.7: Allowed hyperfine transitions for I II. Allowed transitions are constrained by selection rules satisfying $\Delta F=0$ and $\Delta F= \pm 1$. Under each transition is the predicted relative intensity. Reproduced from Ref. [20]; reprinted by permission of the American Institute of Aeronautics and Astronautics, Inc.

mass shift and the specific mass shift. The normal mass shift is kinematical in nature and is composed of the energy possessed by the nucleus and electrons. For a system with $N$ electrons and a nucleus of mass $M$, the normal mass shift is given by

$$
\begin{aligned}
E_{\mathrm{NMS}} & =\sum_{i=1}^{N} \frac{p_{i}^{2}}{2 m}+\frac{\left(\sum_{i=1}^{N} \vec{p}_{i}\right)^{2}}{2 M} \\
& =\sum_{i=1}^{N} \frac{p_{i}^{2}}{2 \mu}
\end{aligned}
$$


where $E_{\mathrm{NMS}}$ is the energy contribution an atom has from the normal mass shift, $p_{i}$ is the momentum of a given particle, $m$ is the mass of an electron, $M$ is the total mass of the isotope, and $\mu$ is the reduced mass in the center of mass frame. The energy difference between two isotopes is a direct result of using Eq. 3.20 with two nuclear masses of $M$ and $M^{\prime}$. A good approximation for the mass difference effect for two isotopes is $\delta \nu / \nu=-\Delta(m / M)$ [72]. The second term, called the specific mass shift, is a result of the correlation of momentum between electrons. The momentum-correlation energy is

$$
E_{\mathrm{SMS}}=\frac{1}{M} \sum_{i<j} \vec{p}_{i} \cdot \vec{p}_{j}
$$

Therefore, the entire expression for the shift in energy for isotopes arising from mass (energy interaction) $E_{\mathrm{MS}}$ is

$$
\begin{aligned}
E_{\mathrm{MS}} & =\sum_{i=1}^{N} \frac{p_{i}^{2}}{2 m}+\frac{\left(\sum_{i=1}^{N} \vec{p}_{i}\right)^{2}}{2 M}+\frac{1}{M} \sum_{i<j} \vec{p}_{i} \cdot \vec{p}_{j} \\
& =\sum_{i=1}^{N} \frac{p_{i}^{2}}{2 \mu}+\frac{1}{M} \sum_{i<j} \vec{p}_{i} \cdot \vec{p}_{j} .
\end{aligned}
$$

The terms describing the purely kinematic side of Eq. 3.22 are typically trivial to calculate. The term that determines the momentum correlation is, in many cases, very hard to determine accurately [84]. This can be problematic since this term can be orders of magnitude larger than the normal mass terms. Both mass shift terms become much smaller as elements get heavier [72]. It is in this regime that a second mechanism, the volume shift, dominates.

The volume shift is also sometimes referred to as the field shift. This term dominates for heavy isotopes. The difference in energy between two isotopes for this effect is due to the distribution of charge over the nucleus and its interaction with the most tightly bound electrons (i.e., $s$-electrons). Exact shifts are hard to determine theoretically, but four general features are outlined in Ref. [72] when the volume shift dominates:

1. "The magnitude of the shift depends mainly on the configuration of the two levels, so that is practically the same for different lines of a multiplet. Appreciable shifts are observed 
only when the number of s-electrons differs in the two terms. This leads to the assumption of a term shift caused by s-electrons."

2. "The sign of the shift is obtained correctly on the assumption that the s-electron raises the level of the heavier isotope relative to the lighter one."

3. "The lines of the even isotopes are always arranged in order of their mass numbers, and the separations tend to be of the same order of magnitude for different isotopes in one line. They are not, however, strictly equal but can show trends and even abrupt changes within the sequence of isotopes. The ratios of the shifts between different isotopes are generally the same or nearly the same in different lines of the same element."

4. "The lines of the odd isotopes-or the centroids of their [hyperfine splitting] h.f.s. patterns-do not lie midway between the adjacent even isotopes, but are shifted towards the isotope of lower A. This effect which occurs to a varying degree in different elements is known as odd-even staggering."

Detailed discussions of this isotopic shift effect are outlined in Ref. [72] and [84]. For clarity, Ref. [72] defines light elements as those with atomic mass $\mathrm{A} \leq 30$ and heavy elements those with atomic mass $A \geq 50$. For elements between these two, both mechanisms contribute comparable energy shift effects.

For the species investigated in this work, isotopic shift is only expected to affect krypton and xenon since iodine has one naturally occurring isotope. The isotopic structure of krypton has been described by Galante [30]. However, if these gases are only used for calibration, the isotopic structure is often not of importance. The integrated signal (integrated over the splitting) still provides a high quality reference for absolute density measurements.

\subsection{Doppler Broadening}

Doppler broadening occurs when the absorbing species has some random thermal velocity that creates a shift in the transition (emission) energy. The Doppler shift of a transition is given by

$$
\Delta \omega=\vec{k} \cdot \vec{v}
$$


where $\Delta \omega=2 \pi \nu, \nu$ is the observed shift in frequency from the resonant transition frequency, $\vec{k}$ is the wave vector of the exciting radiation, and $\vec{v}$ is the velocity vector of the absorber [55]. Using $\omega=2 \pi \nu, \vec{k}=(2 \pi / \lambda) \hat{k}$, and $c=\lambda \nu ;$ Eq. 3.23 is transformed to

$$
\begin{aligned}
\Delta \nu & =\nu^{\prime}-\nu_{\circ}=\frac{\vec{k}}{2 \pi} \cdot \vec{v} \\
& =\left(\frac{1}{\lambda_{\circ}}\right) \hat{k} \cdot \vec{v} \\
& =\frac{\nu_{\circ}}{c} v \\
& \Longrightarrow \nu^{\prime}=\nu_{\circ}\left(1+\frac{v}{c}\right),
\end{aligned}
$$

where $\nu_{\circ}$ is the resonant transition frequency. Equation 3.24 is described in literature as the linear Doppler shift. Equation 3.24 is, of course, only true when the term $v / c$ is small. When considering the range of velocities a particle can have, it is necessary to construct a full description of the Doppler shift, where quadratic and higher order terms contribute [55].

From the above, a Doppler width is calculated for the expected broadening due to thermal motion. The velocity distribution function is given by

$$
f(v) \propto \exp \left(-\frac{m v^{2}}{2 k_{b} T}\right)
$$

where $m$ is the mass of the species, $v$ is the velocity of the species, $k_{\mathrm{b}}$ is Boltzmann's constant, and $T$ is the thermal temperature of the species. Solving for $v$ in Eq. 3.24 and inserting it into Eq. 3.25 it is possible to transform the velocity distribution function into frequency space,

$$
f(\nu) \propto \exp \left(-\frac{\left(\nu-\nu_{\circ}\right)^{2}}{\nu_{\circ}^{2}} \frac{1}{2 k_{\mathrm{b}} T / m c^{2}}\right)
$$

where $\nu_{\circ}$ is the resonant transition frequency for the probed transition of deuterium.

From Eq. 3.26 a definition for the standard deviation of the Doppler broadened distribution is determined:

$$
\gamma_{\mathrm{D}} \equiv \sqrt{\frac{\nu_{o}^{2} k_{\mathrm{b}} T}{m c^{2}}}
$$

Defining the standard deviation in this way reduces the distribution function to the standard form of a Gaussian function. Temperature is determined from Eq. 3.27. However, in practice, a 
measured TALIF profile is not immediately fit to extract the temperature. Instead, the measured distribution is fit to the generic definition of a Gaussian in either wavelength or frequency space, then the laser profile contribution is deconvolved from the signal and the residual linewidth is the Doppler-broadened contribution. It is important to note that the quantity that is produced from the fit of the measured profile is the standard deviation of the distribution. The conversion to FWHM of a traditional Gaussian is easily calculated by

$$
\mathrm{FWHM}=2 \sqrt{2 \ln (2)} \gamma
$$

where $\gamma$ is the standard deviation of the distribution. In wavelength space rather than frequency space, the expressions are essentially unchanged. The only difference is that $\nu$ and $\nu_{\circ}$ are replaced with $\lambda$ and $\lambda_{\circ}$, i.e., wavelength and distribution offset wavelength respectively.

Since deuterium is incredibly light compared to many of the gases interrogated by laser induced fluorescence, the thermal spread in frequency is quite large. Thus, the simulated distributions presented in this work span hundreds of $\mathrm{GHz}$ for low to moderate species temperatures. Figure 3.8 shows simulated lineshapes for $0.026 \mathrm{eV}, 0.2 \mathrm{eV}$, and $1 \mathrm{eV}$ absorbers. Note that in this work, neutral temperatures of approximately $0.2 \mathrm{eV}$ were observed.

\subsection{Saturation Broadening}

Another broadening mechanism can occur when more electrons are being excited than are relaxing back to ground state. This type of broadening is referred to as saturation broadening [85]. Generally, for single-photon LIF, simplified models can be used to estimate the saturation effect of a measured line profile $[53,55,85,86]$. However, for TALIF the models are slightly more involved. In both cases, the best method to determine saturation broadening of a transition is to measure the signal intensity as a function of laser energy to pinpoint the saturation threshold. For single photon LIF, the unsaturated integrated signal is proportional to the laser intensity, $\mathrm{I}_{\mathrm{LIF}} \propto I$; for TALIF, the unsaturated integrated signal is proportional to laser intensity squared, $\mathrm{I}_{\mathrm{TALIF}} \propto I^{2}$.

In previous TALIF measurements, the observed saturation threshold often less than $1 \mathrm{~mJ} / \mathrm{pls}$. Magee et al. found the TALIF krypton saturation threshold to be less than $1 \mathrm{~mJ} / \mathrm{pls}$ for gas 


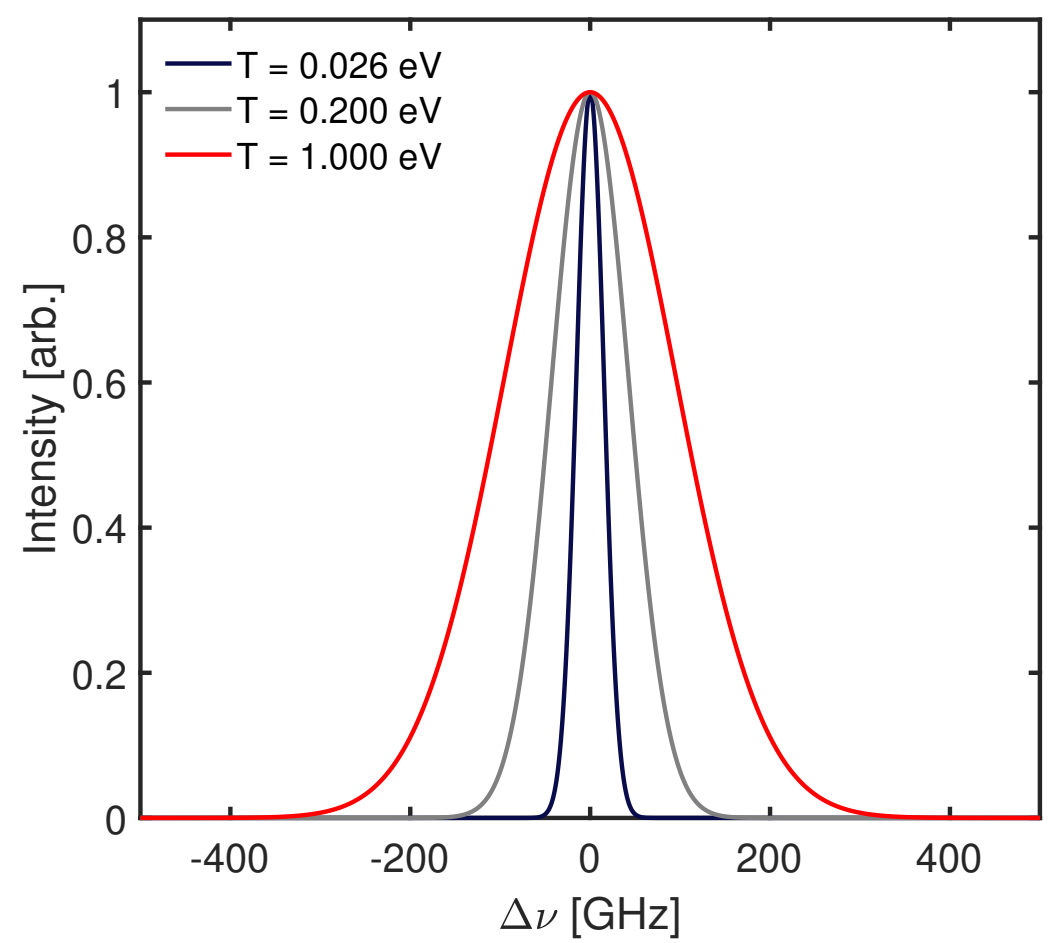

Figure 3.8: Simulated NVDFs for deuterium for room temperature (blue), $0.2 \mathrm{eV}$ (gray), and $1 \mathrm{eV}$ (red). Distributions resembling the temperature of $0.2 \mathrm{eV}$ are observed in this work. All distributions were plotted in the frame of the electron, i.e., $100 \mathrm{~nm}$ frame.

densities on the order of $10^{16} \mathrm{~m}^{-3}$ [43]. Neutral nitrogen NVDFs measured in a microwave discharge afterglow by Mazouffre et al. revealed that saturation thresholds peaked at $\sim 200 \mu \mathrm{J} / \mathrm{pls}$ for neutral densities of $\sim 10^{21} \mathrm{~m}^{-3}[62]$. Es-sebbar et al. observed nearly identical saturation threshold limits in a similar system for similar nitrogen densities [59]. Saturation thresholds for neutral hydrogen were shown to be $\sim 200 \mu \mathrm{J} /$ pls by Amorim et al. [56] Niemi et al. studied saturation limits in both krypton and hydrogen and observed saturation for less than $100 \mu \mathrm{J} / \mathrm{pls}$ [70]. Kajiwara et al. measured substantially larger thresholds for neutral hydrogen saturation at modest densities, but used considerably longer focal length lenses that create significantly lower intensities at the measurement location [87]. For a fixed focal length, the TALIF signal is proportional to the laser energy squared (i.e. $S \sim \mathrm{E}^{2}$ ). Deviation from $\mathrm{I}_{\mathrm{TALIF}} \sim E^{2}$ indicates saturation. While it is common to determine the saturation threshold in terms of the integrated signal, some authors measure saturation from fluorescent intensity [87].

3 pLIF saturation thresholds were investigated by Jain et al. and it was shown that the saturation threshold for this multiphoton process in a combustion system was on the order of $30 \mu \mathrm{J} / \mathrm{pls}$, considerably lower than TALIF experiments. This is likely due to the femtosecond pulse lengths that creates very intense laser pulses. 


\subsection{Summary}

Several broadening mechanisms have been presented and were explored in relation to the species being probed. For single-photon LIF measurements (I II LIF), the measured lineshape is dominated by Doppler and hyperfine broadening. The I II measured lineshape either is not affected by or has negligible contributions from natural lineshape, pressure, Zeeman, isotopic, and saturation broadening effects. For TALIF measurements, the measured lineshapes are dominated by Doppler, laser, and isotopic broadening. Doppler and laser broadening are contributing factors for all three species (deuterium, xenon, and krypton), but isotopic splitting also affects xenon and krypton. As discussed above, the isotopic splitting for the calibration gases is not a source of error as the integration over the measured lineshape provides a valid absolute density reference. Similar to single-photon LIF, TALIF measurements are not broadened by natural linewidth, pressure, Zeeman, or saturation effects. However, unlike I II measurements, TALIF measurements are not broadened by hyperfine splitting. 


\section{Chapter 4}

\section{Experimental Configuration}

\subsection{Iodine Laser Induced Fluorescence}

\subsubsection{Iodine Laser System}

A continuous wave (CW) dye laser system was used in this work to make the first ever LIF measurements of I II. Figure 4.1 shows the optical lasing path of the laser. A Spectra Physics Millenia Pro (MP) neodymium-doped yttrium aluminum garnet (Nd:YAG) CW laser produces up to $10 \mathrm{~W}$ of light at a wavelength of $532 \mathrm{~nm}$ (second harmonic of the fundamental Nd:YAG wavelength, $1064 \mathrm{~nm}$ ). The MP pumps a Sirah Matisse DR laser. The $532 \mathrm{~nm}$ light is first incident on a small mirror, the "pump mirror" (PM), specifically coated for the second harmonic of Nd:YAG lasers. The reflected light is directed to a stream of dye solution emanating from the dye jet (DJ). The dye mixture, the laser gain medium, is forced between two pieces of sapphire at a pressure of 16 bar by a dye circulating pump, resulting in laminar flow of the dye. The DJ is oriented such that light is incident at the Brewster angle. The light excites several rotational and vibrational bands of the dye and emits a broad range of wavelengths specific to the dye mixture. The newly converted light emanates from the dye and is incident on the first folding mirror (FM1). Reflected light passes through a birefringent filter (BiFi) that rejects certain wavelengths of light created from dye conversion. The BiFi acts as a bandpass filter to coarsely determine a lasing wavelength of the laser. Wavelength transmission of the BiFi is dependent 


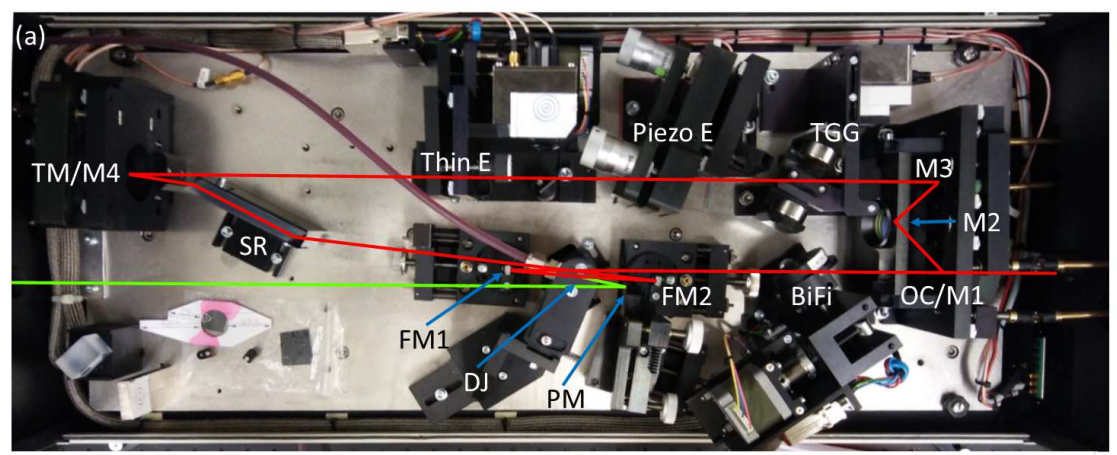

(b)

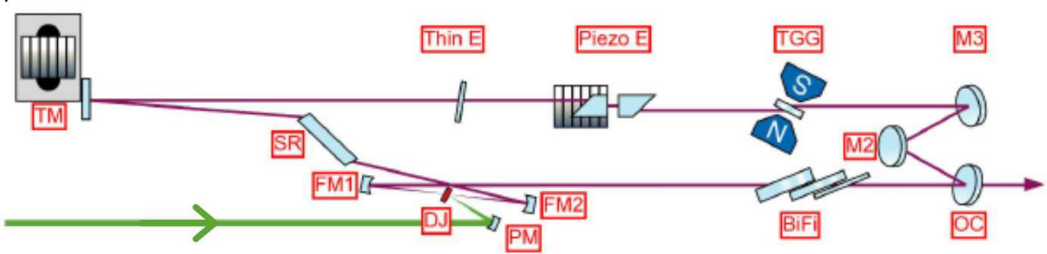

Figure 4.1: Laser path for Sirah Matisse DR. Figure (a) shows beam path overlay for the physical components of the laser and Fig. (b) shows the beam path as depicted in the laser manual [88]. Reproduced with permission. All rights reserved. Sirah Lasertechnik GmbH. In both figures, FM1 is folding mirror 1, FM2 is folding mirror 2, PM is pump mirror, DJ is dye jet, $\mathrm{BiFi}$ is birefringent filter, OC is output coupler (also interchangeably referred to as M1 or mirror 1), M2 is mirror 2, M3 is mirror 3, TGG is Terbium-Gallium-Garnet Faraday rotator, Piezo $\mathrm{E}$ is piezo etalon, Thin $\mathrm{E}$ is thin eltalon, TM is tuning mirror (interchangeably referred to as M4 or mirror 4), and SR is steering rhomb.

on the azimuthal angle of the optic. The $\mathrm{BiFi}$ assembly is rotated by a piezo-driven stepper motor to finely adjust transmission.

The spectrally narrowed light is incident on the output coupler (OC-also referred to as M1 or mirror 1). The output coupler is a mostly reflective, i.e., $>90 \%$, mirror that defines the lasing cavity. The light reflected from M1 travels to mirror 2 (M2). M2 is a highly reflective mirror, $>90 \%$. The small fraction of light transmitted through M2 is monitored by a photo diode for laser power feedback. The reflected light from M2 is incident on mirror 3 (M3) and then passes through a terbium gallium garnet (TGG) plate. The TGG is a Faraday rotation effect optical isolator. Two neodymium magnets are placed on either side of the plate to rotate the polarization of the laser beam so that only light traveling from M3 towards the TGG plate passes through.

After the TGG plate, the light is spectrally narrowed by the piezo etalon (Piezo E), also referred 
to as the thick etalon. The piezo etalon selects a single longitudinal mode of the wavelengths from the wavelength band defined by the BiFi and OC. The final stage of wavelength restriction is provided by the thin etalon (Thin E). The horizontal position of the Thin E is controlled by a piezo stepper motor and spectrally narrows the light to $\leq 20 \mathrm{MHz}$. A small reflection off the window of the Thin $\mathrm{E}$ is directed into a second photo diode that is also used for laser feedback control. The fully spectrally narrow light is reflected off of mirror 4 (M4) and then passes through a steering rhomb (SR) to correct for a beampath offset. After the SR, the light is reflected off folding mirror 2 (FM2) and enters the opposite side of the DJ at the Brewster angle. The light passes through the DJ and is incident on FM1, completing the dye ring.

The overall path length defines the cavity length of the laser head and thus dictates the modehop free range of the laser. The mode-hop free range of the Matisse laser system is specified at $>60 \mathrm{GHz}$. The wavelength is selected by the dye selection, by varying BiFi angle, the Piezo E spacing, and Thin E horizontal angle. Alignment of the Matisse is outlined in Ref. [88], but it should be mentioned that day-to-day alignment for maximum power is achieved by manually tuning M1 and M4. If power is not sufficient after this adjustment, tuning of the Piezo E may be required. A brief outline of Matisse power optimization is discussed in App. B.

Another important note is that since dye lasers have a wide range of possible emission wavelengths it is sometimes necessary to replace M1-M4 with mirrors that have coatings that reflect the desired wavelength ranges produced from the dye. In this sense, the mirrors also act as a crude wavelength filter for possible lasing wavelengths. Lasing wavelengths are a function of the BiFi "order", lasing medium, and mirror set. An order is defined as a set of possible lasing wavelengths permitted as a function of BiFi angle, mirror set, and dye medium. The $\mathrm{BiFi}$ is inserted into the cavity at the Brewster angle and only a select bandwidth of light is transmitted for a given azimuthal angle of the BiFi. All other wavelengths experience losses due to reflections in the BiFi. Figure 4.2 shows the range of wavelengths for different orders.

\subsubsection{Iodine Laser Specifications}

The Matisse laser is well suited for laser spectroscopic measurements of a wide variety of gas species due to its narrow linewidth. The Matisse has a specified laser linewidth $<20 \mathrm{MHz}(<32$ fm) [89], which is comparable to the natural linewidth of many of the transitions of interest in 


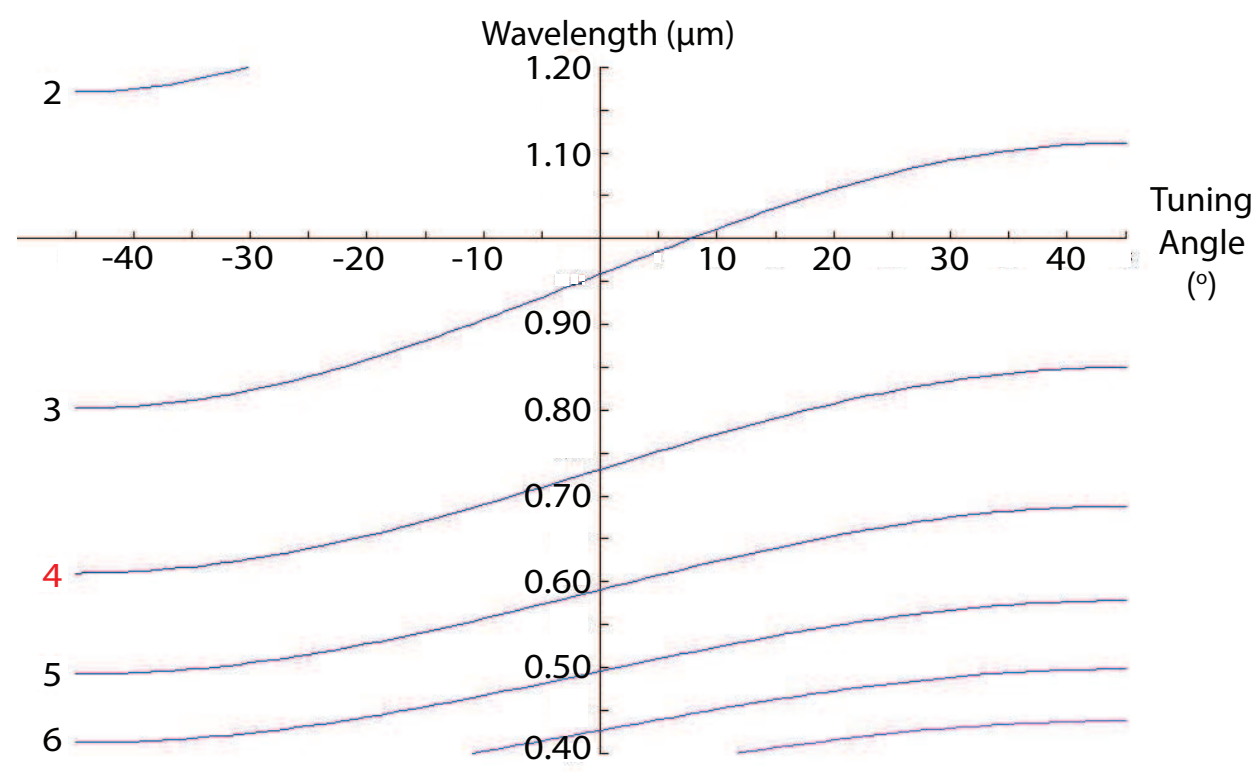

Figure 4.2: Wavelength as a function of birefringent filter tuning angle. Plot provided by Sirah technician. Reproduced with permission. All rights reserved. Sirah Lasertechnik GmbH. Jumping orders may require swapping mirrors in Matisse laser head. Order four is used for the measurements presented here.

common plasmas $[53,86]$. The Doppler-broadened profiles of these species are typically on the order of $\mathrm{GHz}$ and therefore the laser linewidth of the contributes negligibly to the measured profile. The laser beam diameter is specified to be approximately $1.4 \mathrm{~mm}$ (typical) with a beam divergence of less than $1 \mathrm{mrad}[89]$. The small beam diameter offers the benefit of direct injection without focusing optics while providing spatially localized measurements (i.e., $\left.\Delta d_{\max } \sim 1.4 \mathrm{~mm}\right)$.

For measurements of I II, LDS698 (pyridine 1) was mixed with ethylene glycol (EG) and glycol monophenyl ether $(\mathrm{EPH})$ to create a dye solution that has a lasing wavelength range of $670-760$ $\mathrm{nm}$ [88]. The dye recipe for this dye solution is given in App. C. Figure 4.3 shows various dye curves for different dye mixtures, i.e., possible lasing wavelengths for a given dye and intensity of lasing power [89]. It should be noted that the dye curves presented are for a different laser pump power than used in this work and therefore the maximum powers for each dye are not well represented; however, the observed shape of the the curve is similar. For a pyridine 1 solution, the farthest right curve in Fig. 4.3, the Matisse generates $\lesssim 400 \mathrm{~mW}$ of power at peak wavelength. Typically $\sim 200 \mathrm{~mW}$ of power was generated at the wavelengths needed to investigate singly ionized atomic iodine. 


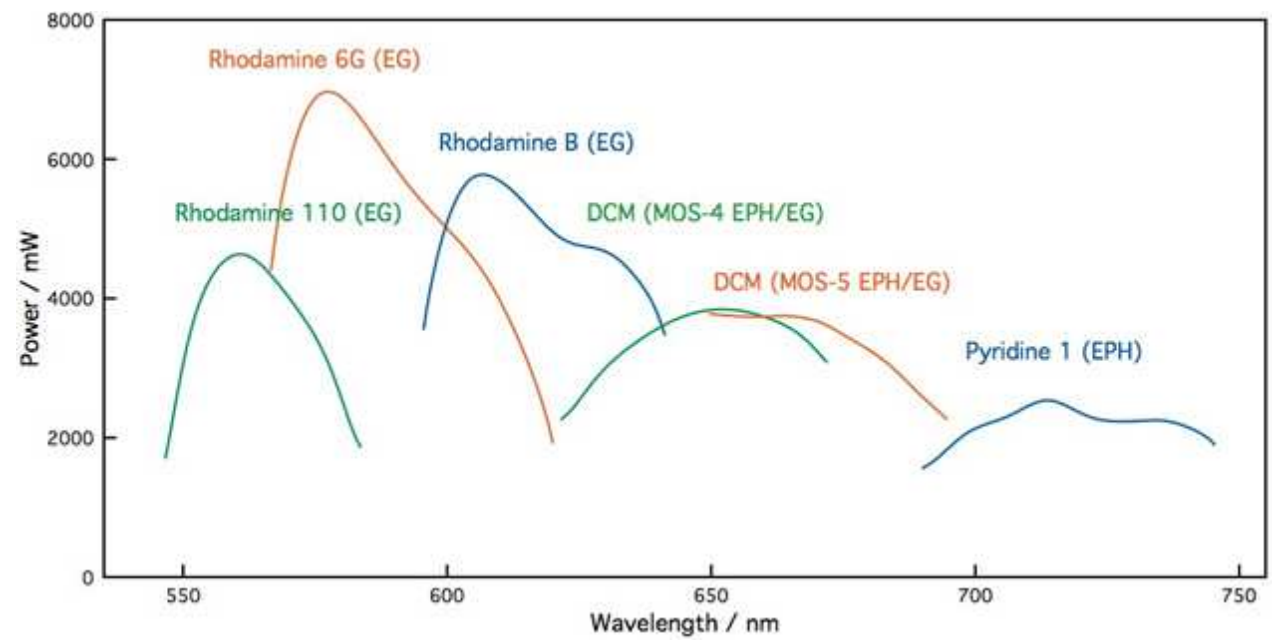

FiguRe 4.3: Tunable range for the Matisse DR [89]. Power depicted is not indicative of power ranges for the model Matisse used in this work, as this plot assumes a higher power pump laser. However, the curve shapes are similar for all pump lasers. Reproduced with permission. All rights reserved. Sirah Lasertechnik GmbH.

\subsubsection{Iodine Data Acquisition (DAQ) System and Layout}

The experimental layout for iodine LIF is shown in Fig 4.4. A small fraction of light exiting the

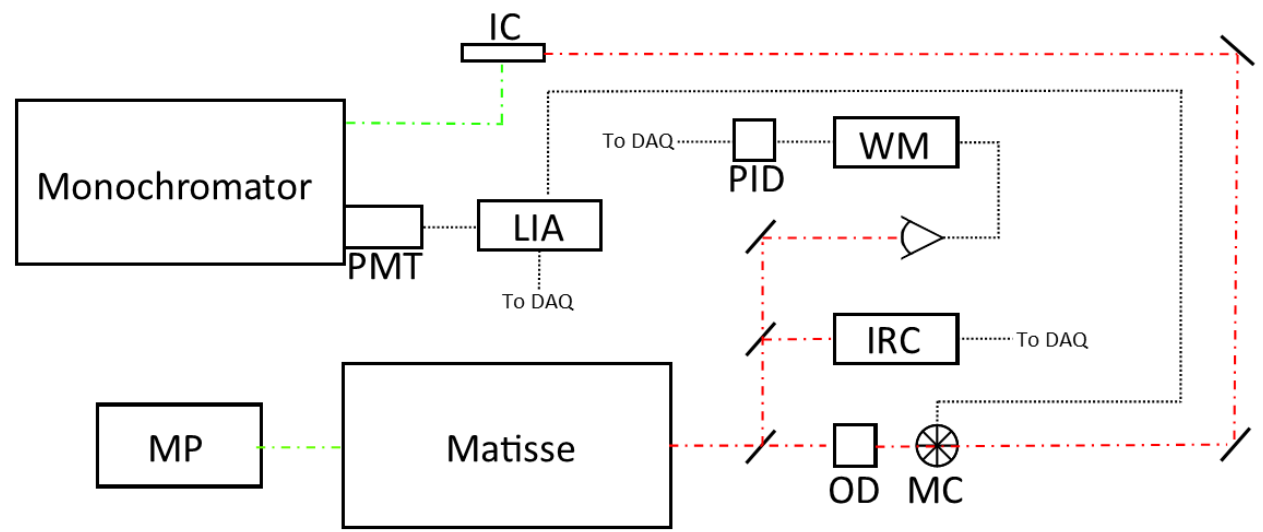

FIGURE 4.4: Experimental configuration for iodine LIF (described in text). Abbreviations: Millenia Pro (MP), optical diode (OD), mechanical chopper (MC), iodine reference cell (IRC), Bristol wavelength meter (WM), custom proportional-integral-derivative (PID) controller, iodine plasma cell (IC), Stanford Research Systems SR830 lock-in amplifier (LIA),

Hamamatsu 10493 photo-multiplier tube (PMT). Reproduced from Ref. [20]; reprinted by permission of the American Institute of Aeronautics and Astronautics, Inc.

laser head $(\lesssim 10 \%)$ is split from the main beam using a thin blank optic and sent to a second thin blank optic where $\lesssim 10 \%$ of the split light is directed through a stationary room temperature iodine reference cell. While an iodine reference cell is often desirable for absolute calibration of bulk flow measured with LIF, there were no detectable transitions for the wavelength span used here. The remaining light is incident upon a third beam splitter where $\sim 10 \%$ is injected into a 
$5 \mu \mathrm{m}$ diameter $(\varnothing 5 \mu \mathrm{m})$ single mode fiber and coupled to a Bristol 621 wavelength meter (WM). The WM monitors laser emission with an absolute accuracy of $\delta \lambda=0.20 \pm 0.03 \mathrm{pm}$ [90]. The digital output of the wavelength meter is recorded using a custom Bristol PID controller that converts the wavelength to an analog voltage between 0 and $5 \mathrm{~V}$ with a user-defined central setpoint. For this work, the setpoint is set to the pump transition wavelength.

The bulk of the laser emission is transmitted through an optical diode to prevent back reflections into the cavity. Efficiency of this diode is specified to be $73 \%-75 \%$, but has been observed to be closer to $80 \%$ during experiments [6]. Immediately after the optical diode is a Thorlabs MCF160 metal chopping wheel controlled via a Thorlabs MC2000B optical chopper controller. The spacing between fins in the metal chopping wheel allows for a tunable chopping frequency of $120 \mathrm{~Hz}-6 \mathrm{kHz}$. The optical chopper provides a reference frequency for the lock-in amplifier to distinguish LIF signal from spontaneous background emission. Two steering mirrors direct the modulated beam down the axis of the iodine plasma device (Sec. 4.1.4). Laser emission is direct-injected into the plasma. Fluorescence is collected using a simple set of $\varnothing 1$ " optics coupled to a $\varnothing 1 \mathrm{~mm}$ core multimode fiber. The fiber transmits light to a McPherson Model 209 1.33 m Czerny-Turner scanning monochromator. For this work, the scanning monochromator acts as a $\pm 5 \mathrm{~nm}$ bandpass filter. To this end, the monochromator has a substantially larger acceptance range than most bandpass filters used in similar works [53, 91, 92]. However, minimal transition "pollution" is visible on either side of the pump transition and fluorescence (see Fig. 5.2), leading to a negligible difference in signal-to-noise (SNR) for these measurements compared to using a narrow bandpass filter. Fastened to the output of the monochromator is a Hamamatsu 10493 photo-multiplier tube (PMT) detector. Discernible signal is differentiated from background emission using a Stanford Research Systems SR830 lock-in amplifier that references the $5 \mathrm{kHz}$ chopping frequency. Filtered output from the lock-in amplifier along with the wavelength determined from the wavelength meter-PID setup is digitized with a National Instruments multi-channel DAQ card for raw data analysis.

For reliable laser spectroscopy results, it is important the ratio of the length of time that the laser scans over a subset of wavelengths and a filtering parameter, called the "time constant" are kept (1) constant and (2) at a value such that oversampling does not contribute to the measured lineshape [93]. For very small values of this ratio, the convolution of the signal and 
filter instrument function introduced by the lock-in amplifier produce an artificial shift in the spectral center of the probed transition, as well as artificial broadening. Inversely, for sufficiently large values, the convolution essentially has no influence on the measured spectral center. The goal is to find the minimum ratio that introduces no error in the measured lineshape for the most efficient measurements. Figure 4.5 shows the spectral center of the probed iodine transition as a function of the ratio of scan time $t_{\text {scan }}$ to the time constant $\tau$. For small values of $t_{\text {scan }} / \tau$, large artificial shift in the spectral center of a measured lineshape is observed. As the ratio of $t_{\text {scan }}$ to $\tau$ becomes larger, the spectral center asymptotes to a non-artificially shifted frequency location. However, if an optimum ratio is not utilized, the true signal can still be recovered. The motivated reader is directed to Ref. [93] for details regarding this process. A ratio of $t_{\text {scan }} / \tau=150$ was chosen as the standard constant ratio for reliable results at the most efficient rate as suggested by Fig. 4.5. Error in Fig. 4.5 is dominated by shot-to-shot variation and accuracy of the wavelength meter.

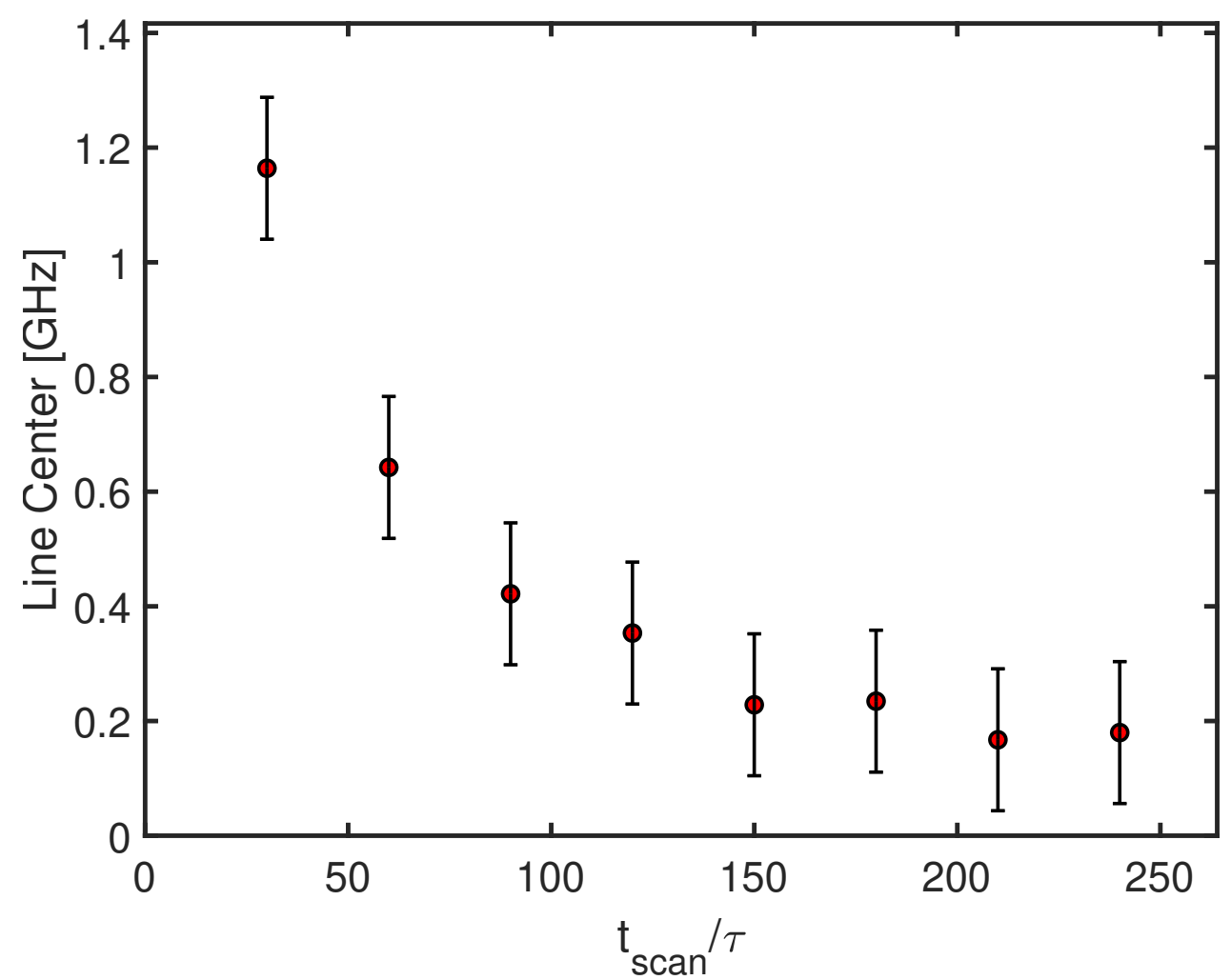

FiguRE 4.5: Measured spectral center as a function of the ratio of laser scan time $t_{\text {scan }}$ to lock-in time constant $\tau$. For small ratios, artificial spectral shift is observed. Artificial shift is negligible for ratios above 150 . 


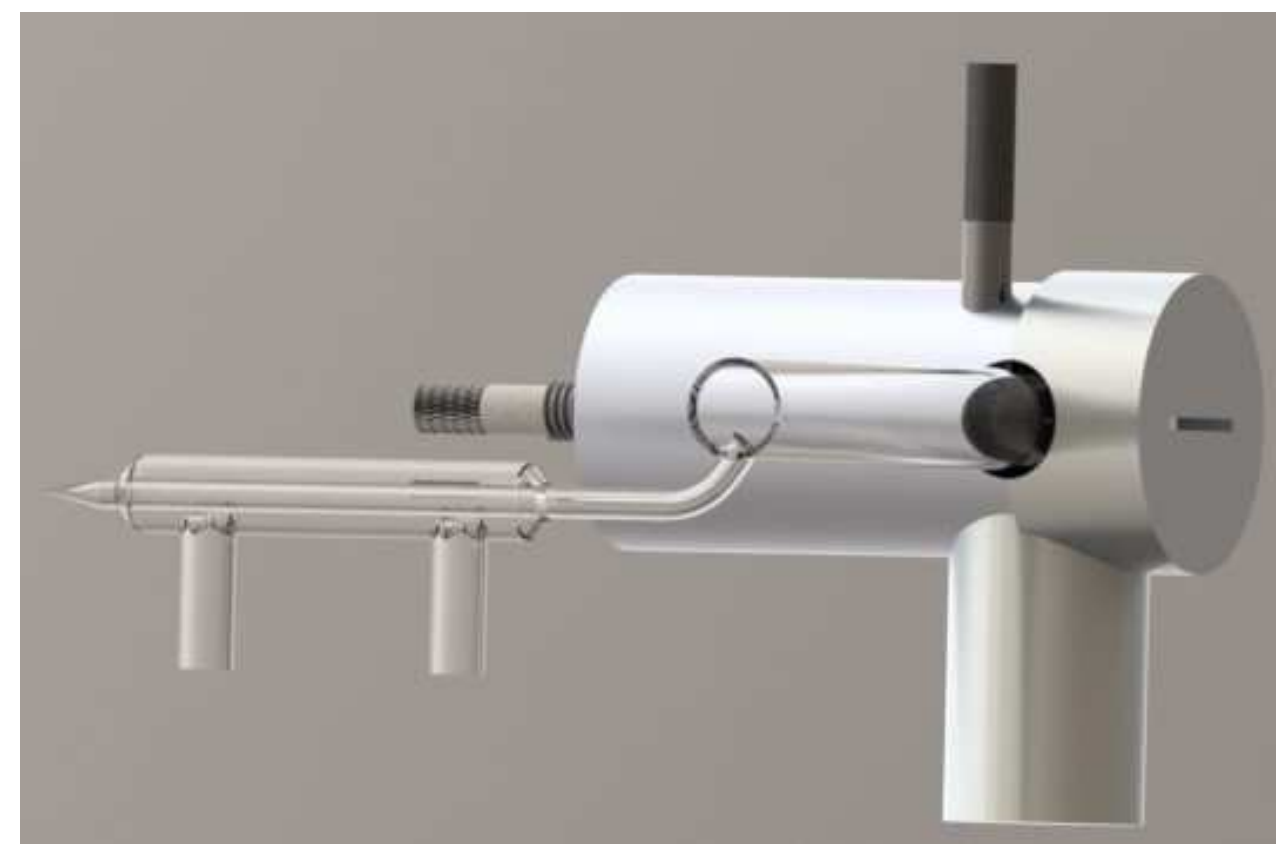

Figure 4.6: Iodine plasma source used in this work. A quartz cell designed with a chilling finger is sealed with a static pressure of $<10^{-4}$ Torr. The cell is inserted into an Evenson microwave cavity that is responsible for maintaining the plasma after ignition.

\subsubsection{Iodine Water-cooled Reference Cell}

Iodine is extremely reactive with many materials since it is a halogen and so laboratory testing of iodine is difficult because of deposition on and erosion of common vacuum hardware [20]. For this reason, a custom static reference cell was designed and built by Opthos Instruments. Figure 4.6 shows a rendered image of the iodine plasma cell. The cell has been described in previous works $[20,22,94]$ and those details are repeated here. Quartz is used for the housing as it is robust to rapid temperature changes and is transparent to both the pump and fluorescence wavelengths. The main cell chamber is approximately $\varnothing 12.5 \mathrm{~mm}$ in diameter and $\sim 100 \mathrm{~mm}$ in length. Jutting out from the main cell is a $\varnothing 6 \mathrm{~mm}$ diameter, $50 \mathrm{~mm}$ long quartz tube. A concentric quartz tube surrounds the majority of this "finger" but is isolated from the vacuum of the cell. The outer cylinder has two stems that allow for coolant to flow between the finger and outer jacket.

A small amount of solid iodine is located inside the main cell and the cell is sealed at a pressure of less than $10^{-4}$ Torr [22]. The iodine cell is placed inside an Evenson microwave cavity. Microwaves are the driver of dissociation of molecular iodine and maintain the iodine plasma once sparked. A microwave power range of $0-100 \mathrm{~W}$ is possible from the microwave power 
supply. A standard handheld high-voltage Tesla coil is used to ignite the plasma. Two tuning stubs allow for optimal power coupling to the plasma. The first tuning stub is a standard screw that is manually driven in and out of the cavity. The second tuning stub is a ceramic rod that traverses the diameter of the microwave cavity, but is offset from the mechanical axis so that it does not touch the iodine cell. It is necessary that this part be made of ceramic to avoid arcing between the cell and tuning stub.

Two separate chillers are used in this work to achieve different operating temperatures of the iodine cell. For initial passive spectroscopic and LIF measurements, a Neslab Thermoflex 960 chiller was used to cool the plasma source. This chiller has a temperature range of $5^{\circ}-40^{\circ}$ C. For measurements that included a static background magnetic field, a second, more robust Polyscience PD07R -40 chiller with a temperature range of $-20^{\circ}-+100^{\circ} \mathrm{C}$ was used. The chiller and quartz concentric extension from the main plasma source combo is essential to control the partial pressure of iodine inside the cell. A similar cell was investigated by Kono et al. and it was found that the partial pressure of iodine $P$ has a dependence on the "chilling finger" temperature $T_{\mathrm{CF}}[95]$ given by

$$
\log _{10} P=18.8-\frac{3954}{T_{\mathrm{CF}}}+0.00044 T_{\mathrm{CF}}-2.98 \log _{10} T_{\mathrm{CF}}
$$

where $P$ is in Torr and $T_{\mathrm{CF}}$ is in $\mathrm{K}$. The range of accessible chilling temperatures correspond to a range of pressures of $\sim 5$ mTorr $-\sim 50$ Torr. For the majority of measurements presented in this work, $T_{\mathrm{CF}}$ was $\leq 5^{\circ} \mathrm{C}(\lesssim 52 \mathrm{mTorr})$. While both the pump and fluorescence transitions are visible with passive emission spectroscopy for $T_{\mathrm{CF}}>20^{\circ}$, the intensity for both transitions increases as temperature decreases (Chapter 5).

As mentioned above, a second configuration of the iodine cell was used to investigate I II in the presence of an external magnetic field. Two permanent magnets surround the iodine cell to create a magnetic field perpendicular to the mechanical axis of the cell. Figure 4.7 shows the layout of the magnetic iodine cell configuration. The two permanent magnets are fastened to an iron yoke that allows for a variable position of the magnets with respect to the iodine cell. The yoke is a necessary component to this design as it guides the magnetic flux to be along the yoke axis. By equally spacing the magnet above and below the iodine cell, the strength of the 


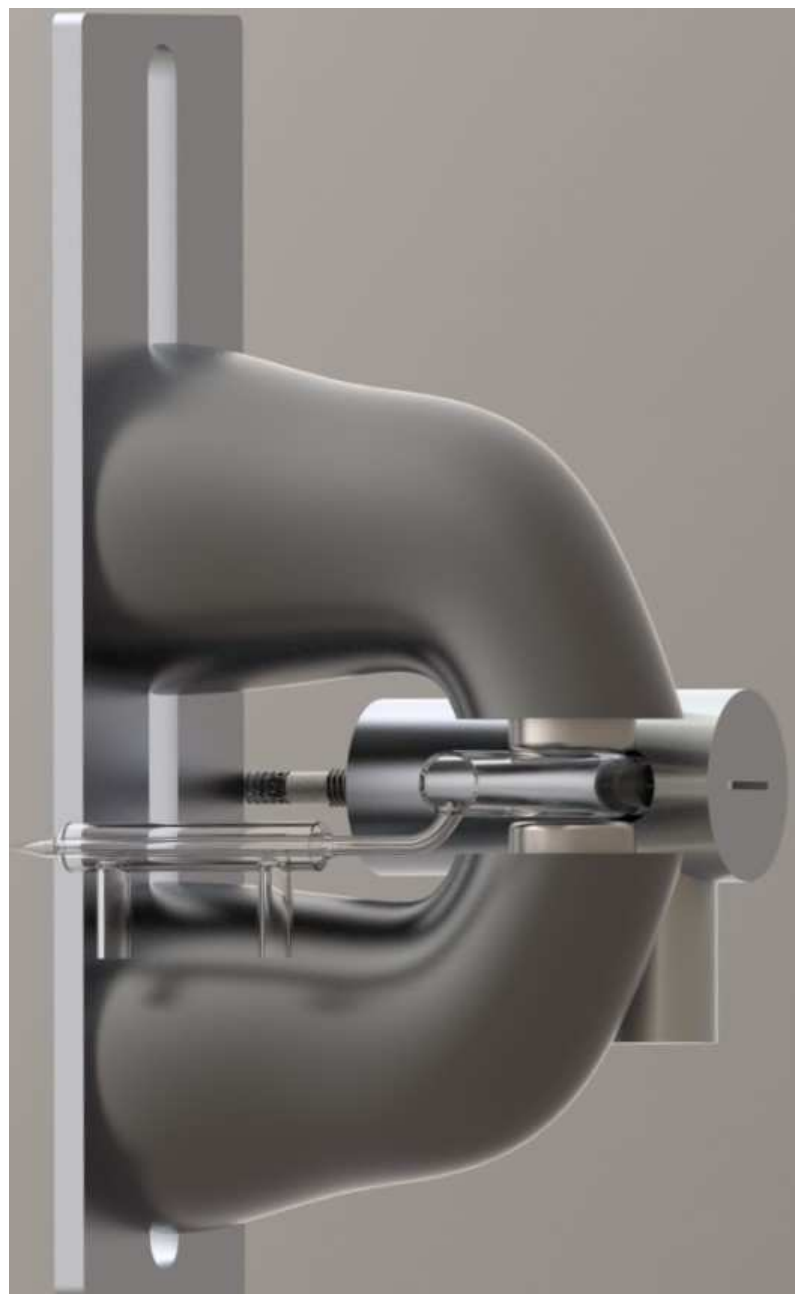

FIGURE 4.7: Iodine plasma source with addition of two identical movable neodymium magnets located above and below a section of the cell. The spacing between the magnets creates a continuous range of magnetic field strengths.

magnetic field is changed. An approximate range of $1-3 \mathrm{kG}$ yielded distinguishable Zeeman split peaks. Details of the Zeeman-split iodine measurements are discussed later in this work.

\subsection{Deuterium Two-photon Absorption Laser Induced Fluores- cence}

A separate laser system was used to probe neutral deuterium than the one described above. A two-laser system is used to create the required ultra-violet (UV) light necessary to probe neutral deuterium. A Quanta Ray Pro 270 pumps a Sirah Cobra Stretch dye laser equipped with a tripling stage. Excess dye stage emission is captured by a $\varnothing 5 \mu \mathrm{m}$ single mode fiber and is coupled to an Ångstrom/High Finesse Ws7-60-UV II wavelength meter that monitors laser wavelength, linewidth, and laser energy. The Ws7 wavelength meter has an absolute accuracy for frequency 
measurements of $60 \mathrm{MHz}(0.08 \mathrm{pm})$ and an absolute linewidth measurement accuracy of 200 $\mathrm{MHz}(2.5 \mathrm{pm})$. Dye laser emission passes through a series of frequency conversion crystals to create UV wavelengths.

The UV laser light is delivered to the Prototype Material Plasma Exposure eXperiment (ProtoMPEX) from $\sim 20 \mathrm{~m}$ away. Injection and collection occur along the same axis using a confocal optical collection design. Fluorescence is detected using a Hamamatsu fast photo-multiplier tube (PMT) and fluorescence is distinguished from spontaneous emission using a Stanford Research Systems Box Car Integrator and Averager. The laser system, beamline, confocal design, and TALIF data acquisition are described in detail below.

\subsubsection{TALIF Laser System}

The two-laser system used in this work is the same as described in Refs. [30] and [96]. A Spectra-Physics Quanta Ray Pro 270 pulsed Nd:YAG laser operating at $20 \mathrm{~Hz}$ is used to pump a Sirah Cobra Stretch dye laser with a tripling stage.

The Quanta Ray pump laser is an Nd:YAG pulsed laser capable of producing $\sim 1200 \mathrm{~mJ}$ of energy per pulse at $1064 \mathrm{~nm}$ (fundamental wavelength). The fundamental emission is frequency doubled and yields approximately $600 \mathrm{~mJ} /$ pulse of energy at $532 \mathrm{~nm}$. Figure 4.8 shows the path of the pump beam through the Sirah dye laser.

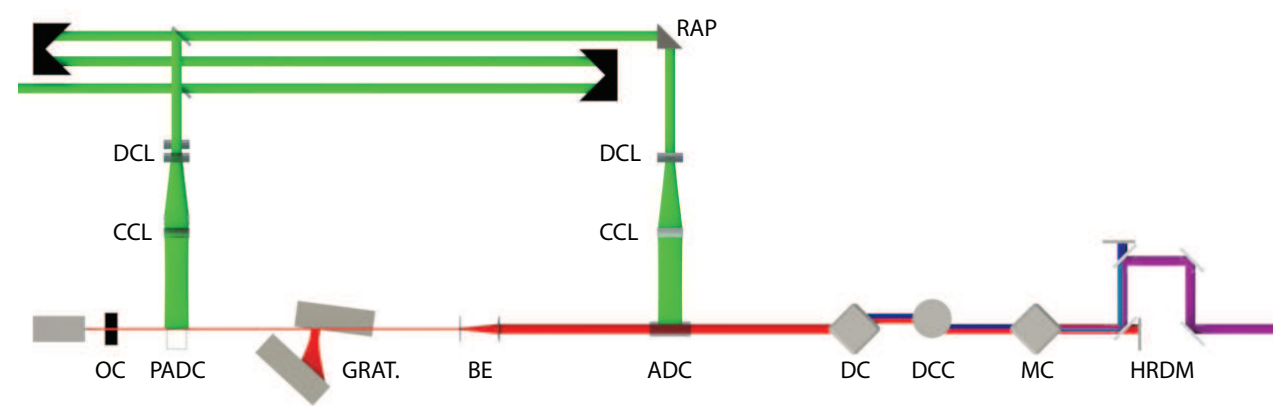

FIGURE 4.8: Laser beam path showing all frequency conversion through the Sirah Cobra Stretch dye laser. The beam path is described in detail in the text. Abbreviations: Diverging cylindrical lens (DCL), converging cylindrical lens (CCL), output coupler (OC), pre-amplification dye cell (PADC), gratings (Grat.), right-angle prism (RAP), amplifier dye cell (ADC), doubling crystal (DC), doubling crystal compensator (DCC), mixing crystal (MC), and high reflective dichroic mirrors (HRDM). 
The frequency doubled light enters the dye stage of the Cobra Stretch and passes through a $95 / 5$ beamsplitter, where approximately $5 \%$ of the light passes through a cylindrical lens arrangement creating a sheet beam that pumps the oscillator section of the rectangular dye cell. The remainder of the pump beam is reflected off four high reflectivity dichroic mirrors and is elevated approximately $1.7 \mathrm{~cm}$. The pump beam passes through another $5 \%$ beamsplitter after the last dichroic mirror where, again, $5 \%$ of the pump beam is directed through a second, identical set of cylindrical lenses creating another sheet beam incident upon the pre-amplification section of the rectangular dye cell at a height $\sim 1.7 \mathrm{~cm}$ higher than the first sheet beam.

The lasing wavelength, typically $\sim 615 \mathrm{~nm}$, is selected by adjusting two 2400 grooves/mm gratings that create a unique cavity length between the output coupler (OC) and the gratings. The small amount of light that transmits through the output coupler is elevated and further amplified by the second sheet beam, producing approximately $25 \mathrm{~mJ}$ of pre-amplified red light. The pre-amplified red light travels through a beam-expanding telescope before it passes through the cylindrical amplifier (capillary) dye cell. The remainder of the pump beam that is transmitted through the second beamsplitter is refracted through a right angle prism, passes through a set of cylindrical lenses, and then pumps the gain medium as the pre-amplifier beam passes through. A maximum of $130 \mathrm{~mJ}$ of energy per pulse is created after the amplification stage.

The amplified red light enters the tripling stage of the Cobra Stretch where it is incident on a second harmonic generation beta barium borate (BBO) crystal with a cut angle of $44^{\circ}$. At optimum laser alignment, $\sim 40 \mathrm{~mJ}$ of frequency-doubled $307.5 \mathrm{~nm}$ light is produced after the first crystal. Not all incident light is converted as some of the dye-converted light $(615 \mathrm{~nm})$ is essential to produce $205 \mathrm{~nm}$ light. The $307.5 \mathrm{~nm}$ and remaining $615 \mathrm{~nm}$ light then pass through a mixing $\mathrm{BBO}$ crystal with a cut angle of $77^{\circ}$ to produce the third harmonic of the fundamental wavelength at $205 \mathrm{~nm}$.

The UV wavelength is $1 / 3^{\text {rd }}$ the measured wavelength at the dye stage. The High Finesse also measures the laser power and laser linewidth at the dye stage. However, the laser linewidth measurement only yields a value in "coarse" mode and toggles uncontrollably between "underexposed" and "overexposed" in "fine" mode. The coarse mode measurement produces a value approximately 3 times larger than the vendor's specification. For this reason, the vendor's value has been used rather than the coarse measurements. 


\subsubsection{TALIF Laser Specifications}

A maximum of $5 \mathrm{~mJ}$ of energy at $205 \mathrm{~nm}$ is produced in a $\sim 8 \mathrm{~ns}$ pulse with a beam diameter of $\sim 5 \mathrm{~mm}$ (specified). The beam diameter was measured using a razor blade fastened to a linear motion stage. As the razor edge passes through the beam, energy measurements were made until the entire beam was blocked. Figure 4.9 shows energy as a function of linear position of the razor blade (a) and its derivative in (b). The derivative is the spatial profile of the laser beam and is well described by a Gaussian function. There are three common methods of estimating the beam diameter from the profile data:

1. Full-width at half-maximum of the laser beam profile

2. $1 / e^{2}$ width (referred to as $\mathrm{D}_{\text {Beam }}$ )-approximately $86 \%$ of the laser beam energy is contained in this measurement

3. $4 \sigma$ width-distance between 4 standard deviations.

The results of all three methods are shown in Fig. 4.9 (b). The specified beam diameter is in good agreement with the latter two methods.
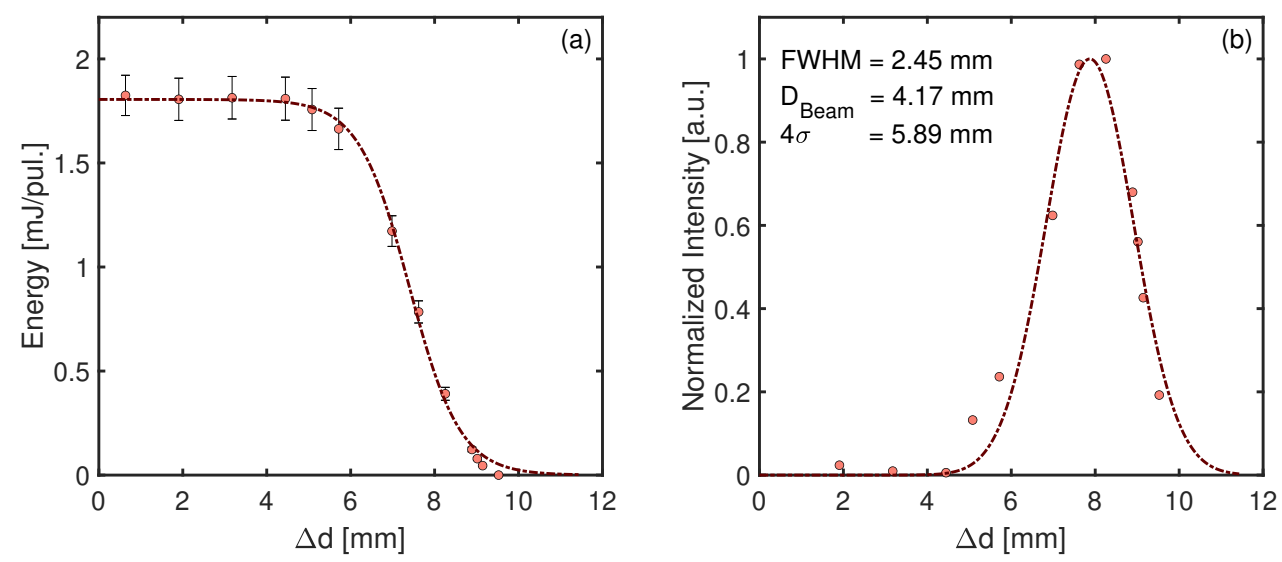

FIGURE 4.9: (a) Energy measured in the UV as a function of razor blade position. (b) The derivative of the measured energy as a function of razor blade position. The effective beam diameter calculated from three common methods is displayed in (b). The vendor's specification of beam diameter is in good agreement with methods 2 and 3 .

A key laser parameter for TALIF measurements is the energy per pulse. Dominating the energyper-pulse is the conversion efficiency of the dye medium across the desired wavelengths. For the specific recipe used in this work, the lasing range is approximately $40 \mathrm{~nm}$. The TALIF 


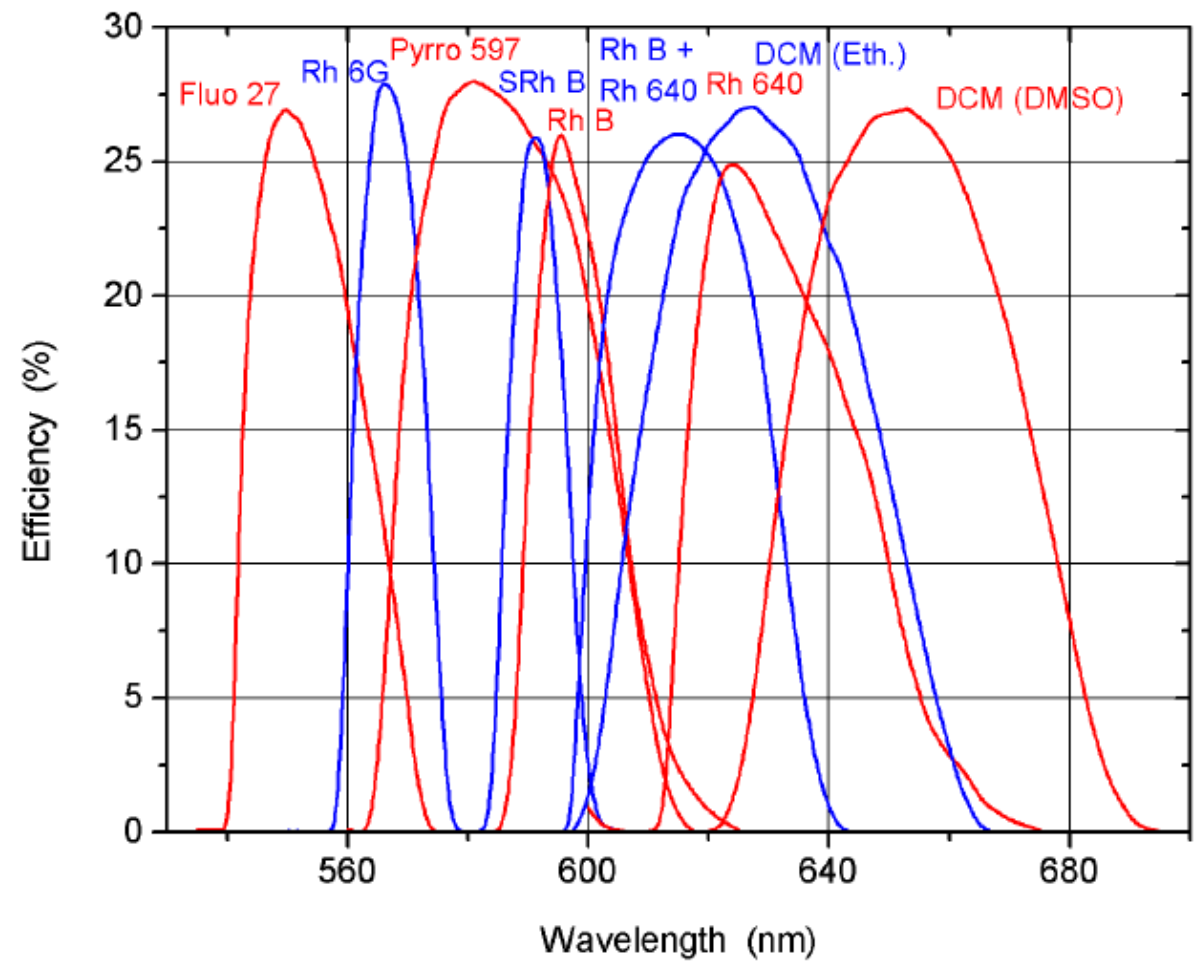

Figure 4.10: Several dye curves for Sirah Cobra Stretch dye laser. The dye mixture used in this work is shown by the blue " $\mathrm{Rh} \mathrm{B}+\mathrm{Rh} 640$ " curve. This mixture peaks at $615 \mathrm{~nm}$ with approximately $27 \%$ efficiency. Figure reproduced from Ref. [71] with permission. All rights reserved. Sirah Lasertechnik GmbH.

laser system dye curve, shown in Fig. 4.10, is reproduced from Ref. [71]. The recipe for the dye mixture is given in App. C and is highlighted here. A combination of Rhodamine B and 101 (Rhodamine 610 and 640 respectively) are mixed with ethyl alcohol (ethanol) to produce a lasing wavelength range of $598-636 \mathrm{~nm}(\sim 200-212 \mathrm{~nm}$ after tripling stage $)$ with a peak efficiency at $615 \mathrm{~nm}(205 \mathrm{~nm})$ with a maximum conversion efficiency of $27 \%$. As mentioned in Sec. 4.2.1, the TALIF laser system utilizes two dye conversion stages, one for initial creation and pre-amplification of the laser pulse and a second for amplification of the pulse. While the same dyes are used for both the pre-amplification and amplification stages, the dye solution in the amplification stage is diluted to $1 / 8^{\text {th }}$ the concentration of that in the pre-amplification stage. Since the doubling and mixing crystals have non-linear efficiency over the dye emission wavelength range, a peak conversion in the dye curve does not necessarily translate to a peak in the UV energy. Typically, a small fraction of UV light is split from the laser emission and is directed to an energy meter for real-time measurements of the laser pulse energy for signal normalization $[58,61,96]$. To avoid any reduction in the laser energy injected into the plasma 
during plasma measurements, the pre-tripled energy-per-pulse was recorded by the wavelength meter. An energy conversion table for the range of wavelengths that span a neutral velocity distribution function (NVDF) was created to estimate the UV energy-per-pulse delivered to the plasma. An energy conversion table was created by manually stepping the laser through the wavelength range for $\sim 1000$ pulses while measuring the pulse energy with the wavelength meter and a Gentec energy meter placed at Proto-MPEX. Energy conversion curves for the wavelength range appropriate for each target species are given in App. D.

Detailed knowledge of the laser linewidth is a crucial factor in obtaining accurate Doppler width measurements with TALIF. The laser linewidth immediately after the dye conversion stage is specified to be $0.95 \mathrm{GHz}(1.20 \mathrm{pm})$ by the vendor [71]. As described above, the light created in the dye stage passes through a doubling crystal. For a detailed description of the frequency conversion processes, the motivated reader is directed to references [97] and [98].

Loosely, the doubling crystal can be thought of as acting as a "photon welding device," joining two photons and creating a single new photon with twice the energy and half the wavelength. Physically, the frequency conversion process is a two-photon process that involves absorption of two photons and emission of a single photon with twice the frequency. While the physics of frequency conversion is very important, it is the evolution of the photon lineshape that is most important for TALIF measurements. Mathematically, the process can be described as a convolution of two "dye-stage laser" lineshapes:

$$
f_{307}=f_{615} * f_{615},
$$

where $f_{307}$ is the laser lineshape of the newly created frequency-doubled light and $f_{615}$ is the lineshape of a "dye-stage" photon. Here it is assumed that the laser is set to $615 \mathrm{~nm}$ (the peak of the dye curve). To adhere to common practice, all calculations are presented in frequency space (though the subscripts are labeled with a reference to wavelength for familiarity).

To calculate the lineshape of the doubled wavelength, a simple Fourier transform makes the convolution of two functions a product of the two Fourier transformed functions [99]. Then, the inverse Fourier transform of this product reveals the lineshape after the doubling crystal. The process of determining the laser lineshape after the doubling crystal presents a unique aspect for 
the spectroscopic analysis in this work. A laser lineshape is commonly assumed to be Lorentzian [100]. However, some authors choose a Gaussian laser lineshape as it makes deconvolution mathematically simpler [30, 96]. While assuming the laser lineshape to be Gaussian is generally an acceptable approximation, the approximation becomes less accurate as light is frequency converted because the widths yielded from two convolved Gaussian functions and two Lorentzian functions are different. Gaussian lineshapes convolve together to produce widths that add in quadrature, whereas Lorentzian lineshapes convolve to produce widths that are simple sums (derivations of each of these convolutions are shown in App. A). Hence, while the two lineshapes only differ slightly at the dye stage, they differ significantly after frequency conversion. Since a firm understanding of the laser lineshape is critical for reliable temperature measurements, this work chooses to adopt the Lorentzian lineshape approach. Several variations of Lorentzian profiles exist. In the interest of transparency, this work uses the following definition:

$$
f_{\mathrm{L}}=\frac{\Gamma}{\left(\nu-\nu_{\mathrm{o}}\right)^{2}+\Gamma^{2}}
$$

where $\nu_{\circ}$ is the center frequency of the Lorentzian and $\Gamma$ is the half-width at half-maximum (HWHM) of the lineshape. In most cases, the full-width at half-maximum is used to characterize lineshapes and therefore the relation between the full-width at half-maximum (FWHM) and half-width at half-maximum (HWHM) is

$$
\mathrm{FWHM}=2 \Gamma .
$$

The laser lineshape produced after the doubling crystal has a width that is twice as large as the lineshape at the dye conversion stage. The mixing crystal has a similar influence on the total laser lineshape. After the mixing crystal, frequency-doubled light is convolved with residual dye-laser light and produces a laser lineshape for the $205 \mathrm{~nm}$ photon that has a linewidth that is three times as large as the the linewidth of the dye laser light, that is

$$
\Gamma_{205}=3 \Gamma_{615}
$$

Figure 4.11 illustrates the difference between the frequency converted lineshapes by showing the laser lineshape represented by both a Lorentzian and Gaussian profile at the dye conversion 
stage (a) and at the laser exit (b). It is straightforward to see from (a) the reason it is common to approximate the laser lineshape as Gaussian; deviations are small and are only distinguishable in the wings of the lineshape. However, the lineshape is noticeably different for the two functions at the exit of the laser, i.e., after the frequency conversions-demonstrating the need to use the correct laser lineshape in the convolution analysis.

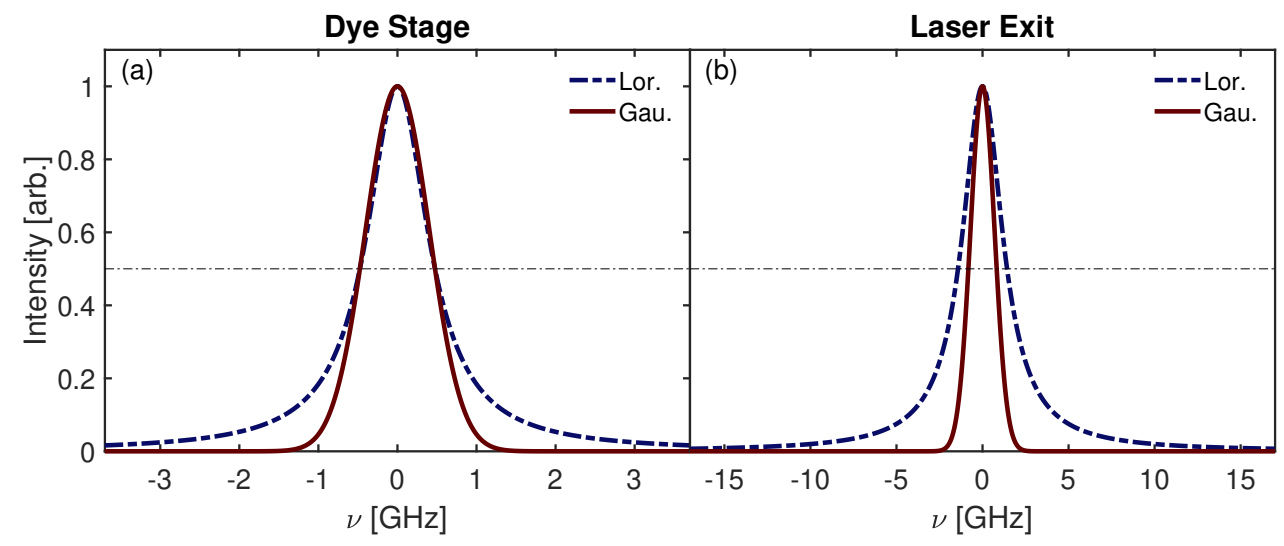

FIgURE 4.11: Comparison of laser lineshapes between Lorentzian (blue) and Gaussian (red). The two functions at the dye stage of the laser (a) are compared with the lineshapes at the laser exit (b). It is apparent on the left that if the laser light did not undergo any further frequency conversions the two profiles are negligibly different. However, since the two lineshapes transform differently under frequency conversions, the two lineshapes differ by a significant amount at the laser exit. The linewidth of a Gaussian profile is a quadrature sum of the input linewidths and the Lorentzian profile linewidth is a sum of the linewidth of input linewidths. Therefore, the Gaussian linewidth in (b) is $\sqrt{3}$ times larger than the linewidth in (a) and the Lorentzian linewidth in (b) is 3 times larger than the Lorentzian linewidth in (a). A black dashed line at 0.5 indicated that half-maximum intensity of the lineshapes.

A summary of the TALIF laser system specifications are listed in Table 4.1. Values with an asterisk were measured. All other values are taken from specified values in the laser manual or from previous works.

\begin{tabular}{lcccc}
\hline \hline & Nd:YAG Stage & Dye Stage & Doubling Stage & Mixing Stage \\
\hline Max. energy-per-pulse (mJ) & 600 & 150 & 40 & $5^{* 1}$ \\
Peak Wavelength (nm) & 532 & 615 & 307.5 & 205 \\
Pulse Width (ns) & $1-2$ & $6-8$ & $6-8$ & $\sim 7^{* 2}$ \\
Beam Diameter (mm) & 8 & 5 & 5 & $\sim 5^{*}$ \\
Divergence (mrad) & $<0.5$ & $<0.5$ & $<0.5$ & $<0.5$ \\
$\Delta \nu(\mathrm{GHz})$ & $<32.2$ & 0.95 & 1.90 & 2.85 \\
$\Delta \lambda(\mathrm{pm})$ & $<28.3$ & 1.20 & 0.60 & 0.40 \\
$\Delta k\left(\mathrm{~cm}^{-1}\right)$ & $<1$ & 0.03 & 0.06 & 0.10 \\
\hline
\end{tabular}

TABLE 4.1: Laser characteristics for the TALIF laser system from pump laser to tripling stage. Values with an asterisk indicate that the quantity was measured. In all other cases, values are based on vendor specifications. 


\subsubsection{Ultra Violet Beamline}

Space restrictions near the Proto-MPEX chamber dictate that the TALIF laser system be located in a separate laser room. As a result, UV light must travel nearly $20 \mathrm{~m}$ from this room to the chamber. The power density of the pulsed laser system necessitates a completely enclosed structure that routes the laser emission to the chamber while maintaining a safe working environment. For this reason a beamline was constructed to safely transmit the laser beam. The propagation of the laser beam was modeled with Zemax to understand divergence along the path.

Figure 4.12 shows a rendered image of the beamline constructed for this work. The beamline

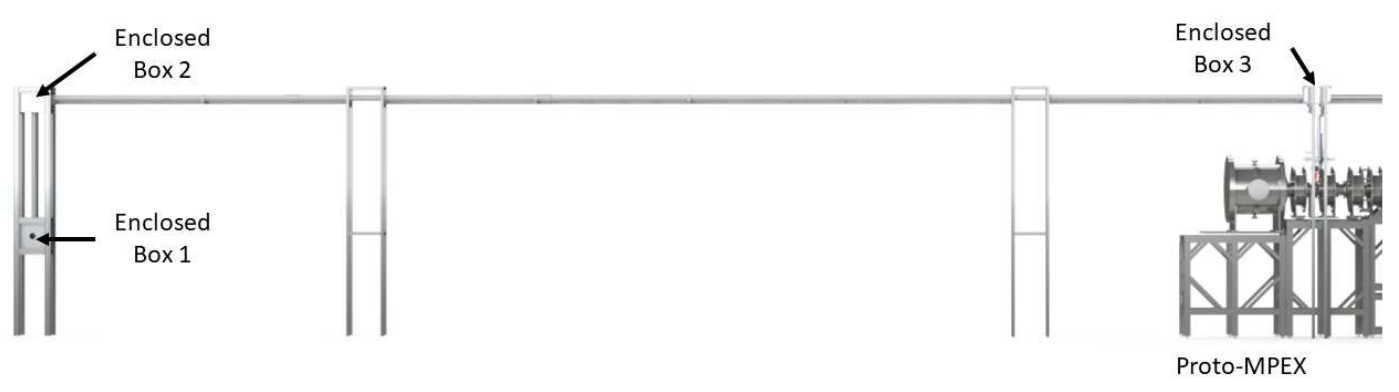

FIGURE 4.12: Rendered image of the UV beamline constructed for this work.

structure consists of three components: a light-tight box constructed from $80 / 20^{\mathrm{TM}}$ located at the exit of the tripling stage (not shown), a tower constructed from $80 / 20^{\mathrm{TM}}$ that consists of two enclosed boxes separated by a distance of $\sim 2 \mathrm{~m}$ that act to send the beam to Proto-MPEX, and a final partial tower with an enclosed box that directs the beam into the vacuum vessel. Each optical enclosure is connected by an appropriate length $\varnothing 3 " \mathrm{PVC}$ conduit that serves to enclose the beam between reflections.

Upon exiting the Cobra Stretch tripling stage, the UV light reflects off two $\varnothing 1$ " protected aluminum, deep UV mirrors located in the first light-tight box. While these first two mirrors are sources of loss (from reflections), they facilitate alignment/coupling into the first optical enclosure housing. Estimated worst-case loss from these two mirrors is $\sim 15 \%$.

\footnotetext{
${ }^{1}$ Laser delivery report indicates $8.5 \mathrm{~mJ} / \mathrm{pls}$ maximum energy after tripling stage. However, only a maximum of $5 \mathrm{~mJ} /$ pls. was observed in this this work.

${ }^{2}$ Measured previously by Galante, Ref. [30].
} 
The beam undergoes three total reflections after the steering mirrors to transit the beamline. First, the beam is reflected $90^{\circ}$ upwards from a $\varnothing 1 "$ diameter UV mirror. The light travels approximately $1.7 \mathrm{~m}$ to the second optical enclosure housing, where the light is incident upon another $\varnothing 1 "$ UV mirror, directing the light towards Proto-MPEX. After approximately $15.5 \mathrm{~m}$, the UV light reaches a final $\varnothing 2$ " UV mirror that redirects the UV pulse approximately $1 \mathrm{~m}$ downwards into the chamber. The total length of the beam path is approximately $20 \mathrm{~m}$. Since water vapor is an absorber of UV light, moisture in the ambient air acts as a considerable loss mechanism for the beam. To mitigate absorption losses along the beam path, the beamline is equipped with a nitrogen purge line, located near the midpoint of the beamline structure. Despite these efforts, only $\sim 20 \%$ energy transmission from the laser exit to the chamber is typical.

Situated adjacent to each beamline mirror (excluding the two initial steering mirrors) is a Thorlabs DCC-1545 camera that is used to monitor the beam position. Ideally, the cameras would monitor the beam position on the mirror in real time; however, the UV pulse cannot be seen on the face of the mirror with the camera, nor can any residual light that leaks through the mirror be detected from behind the mirror. Therefore, a Thorlabs MFF101 motorized filter flipper is placed in front of the mirror that allows for a mirror blank coated with sodium salicylate to be mechanically rotated into the beam path. Sodium salicylate is a UV phosphor. This configuration facilitates initial gross alignment, but cannot be used for real-time monitoring of the laser pulse as the flipper would block the beam. Figure 4.13 shows the standard configuration of the mirror-camera-flipper setup. Once the laser pulse has been sufficiently aligned through the beamline, a fiducial camera image of the beam position on each flipper is cataloged for future gross alignment purposes. The three beamline mirrors, i.e., all mirrors except first two steering mirrors, are held by piezo kinematic mounts, allowing for remote beam direction adjustment. The first two $\varnothing 1 "$ mirrors are held by an Agilis ${ }^{\mathrm{TM}}$ AG-M100N piezo optical mount controlled by an AG-UC2 two-axis motor controller. The final beamline mirror is held by a standard Thorlabs KM200 Ø2" kinematic mount with two PIAK10 $10 \mathrm{~mm}$ piezo inertia actuators that replace the manual adjustment screws. The actuators are controlled by a Thorlabs KIM101 K-cube inertial piezo motor controller. Finally, the laser pulse is shaped through an injection-collection optical apparatus coined as the "confocal optics," described below. 


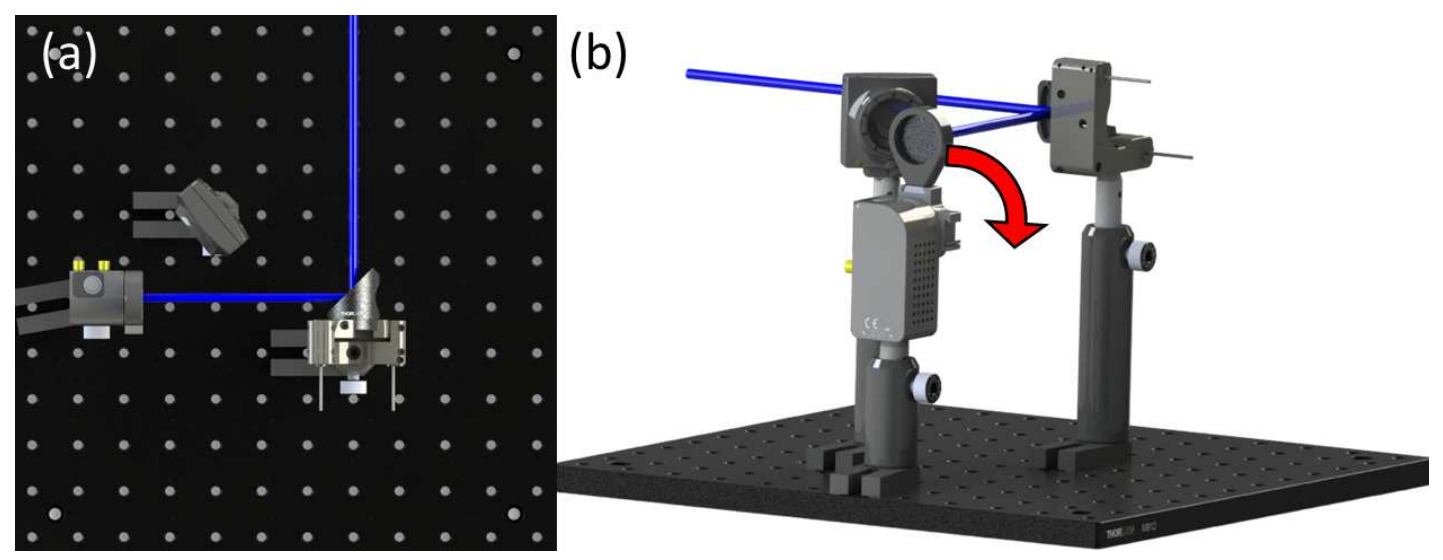

Figure 4.13: Top view of typical mirror-camera-flipper configuration (a) and off-axis view of same setup (b). The red arrow on the right hand side shows the motion of the mechanical filter flipper. A mirror blank coated with sodium salicylate fluoresces from the UV pulse for camera detection.

\subsubsection{Confocal Optics}

Vacuum ports for diagnostic access are at a premium on fusion devices. To minimize port access requirements for TALIF on such machines, fluorescence is collected confocally using the confocal apparatus shown in Fig. 4.14.

A Zemax simulation of the injection beam path predicts that the beam diameter expands from 5 $\mathrm{mm}$ to approximately $27 \mathrm{~mm}$ by the time the beam is incident on the confocal apparatus. For this reason, the first stage of the confocal apparatus consists of a Keplerian-style beam compressor. This compression technique takes the beam diameter from $\sim 27 \mathrm{~mm}$ to approximately $6 \mathrm{~mm}$.

The compressed beam passes through a $12 \mathrm{~mm}$ hole drilled at a $45^{\circ}$ angle through a 2 " protected aluminum coated plane mirror mounted at the center of the 2" cage mountable cube. This is a necessary feature of the confocal design, as it allows injection and collection to exist on the same optical axis. The beam is focused through a $150 \mathrm{~mm}$ focal length $\varnothing 2$ " calcium fluoride $\left(\mathrm{CaF}_{2}\right)$ lens fastened to the bottom of the 2" cage mountable cube.

Fluorescence is collected through the same focusing lens. Collected light reflects off of the mirror held at $45^{\circ}$ and is focused through a $75 \mathrm{~mm} \varnothing 2 "$ lens into a $\varnothing 600 \mu \mathrm{m}$ or $\varnothing 1500 \mu \mathrm{m}$ core multimode fiber. The collection mirror is mounted to an adjustable rotation mount. The rotation mount provides pitch and yaw degrees of freedom, allowing for fine adjustment of fluorescence through the focusing lens into the fiber. 


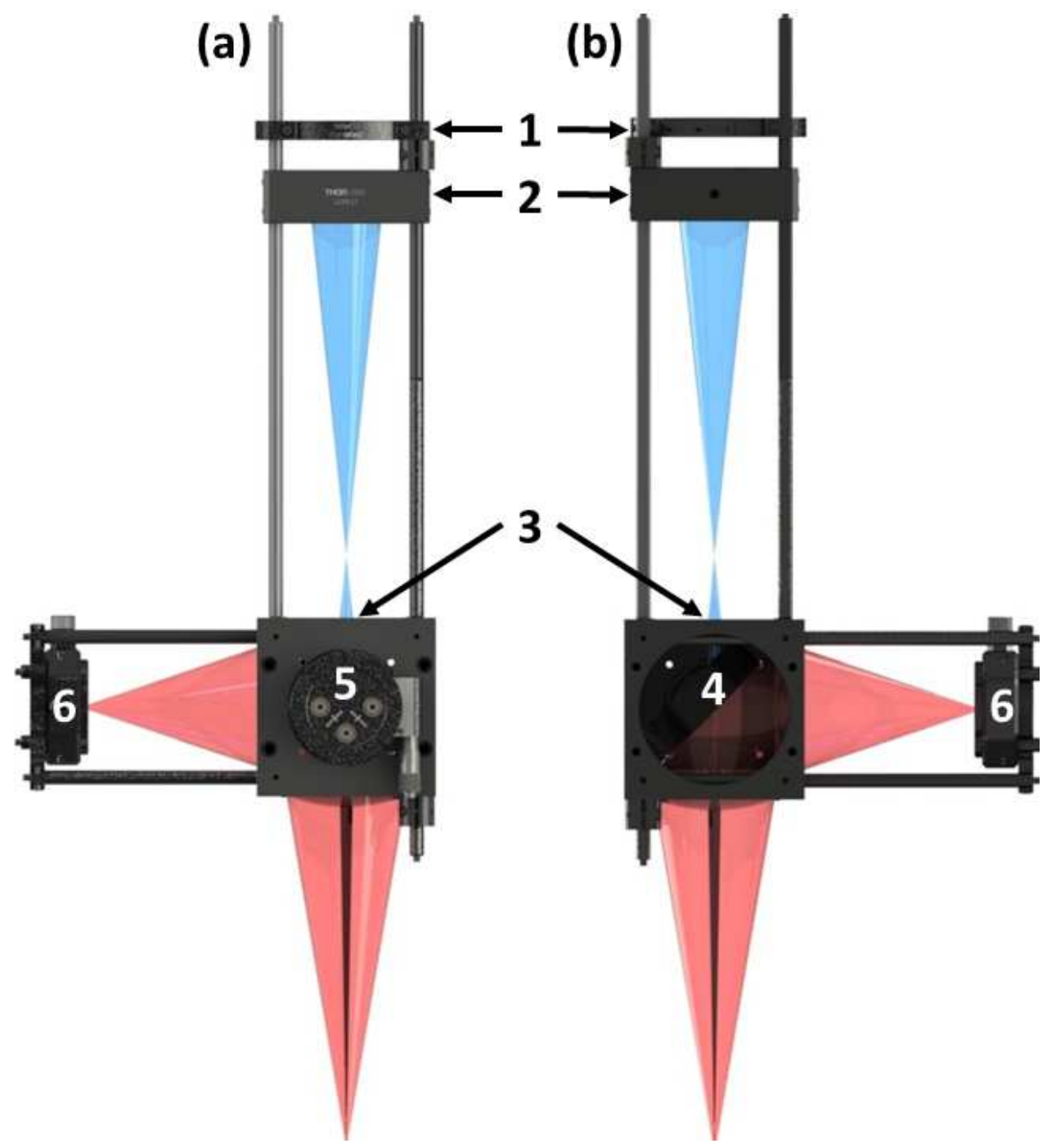

Figure 4.14: Rendered side views of the confocal optical design. UV laser pulse enters from above and passes through a cage mountable variable aperture ("iris") (1). The iris is essential to ensure the laser pulse passes through the center of the confocal apparatus. The divergent laser pulse is then passed through a Keplerian-style optical beam compressor $(2,3)$ (described in text). The compressed beam travels through a $\varnothing 2 "$ mirror with a $12 \mathrm{~mm}$ hole drilled at an angle of $45^{\circ}(4)$. The mirror is mounted at $45^{\circ}$ on a high-precision rotational mount (allowing for pitch adjustment) with yaw control (5). Fluorescence is collected with the remaining surface area of the mirror and is focused into an "X-Y" translatable cage mount (6) coupled to a multimode collection fiber. A second iris is placed at the bottom of the confocal apparatus for alignment, but is removed for on-axis measurements.

The confocal system is mounted to a series of linear and tilt stages that allow for gross alignment of the optical apparatus axis to the beam propagation axis. To ensure that the beam is passing through the collection apparatus parallel to the axis of the structure, cage-mountable irises are placed at the top and bottom of the cage system. However, to have the confocal optics close enough to probe measurements on-axis of Proto-MPEX, the bottom iris is removed after initial alignment. 


\subsubsection{TALIF Electronics and Experimental Layout}

Figure 4.15 shows a layout of the electronics used for deuterium TALIF experiments. The electronics used for calibration are slightly different and are discussed next. An initial trigger

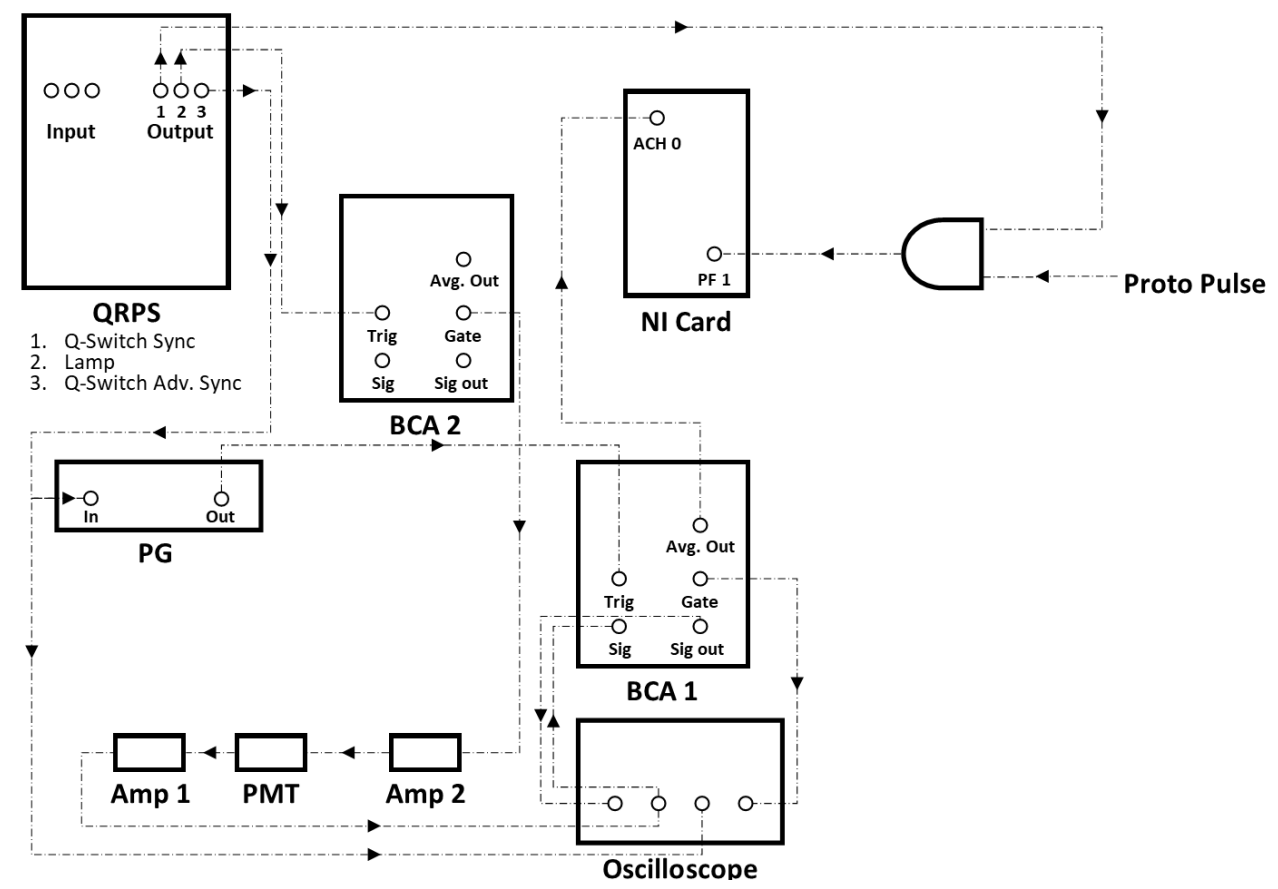

FiguRE 4.15: TALIF electronic layout for deuterium measurements (described in text). Abbreviations: Quanta Ray power supply (QRPS), National Instruments BNC-2110 BNC adapter DAQ card (NI Card), Stanford Research Systems SR250 Gated Integrator and Box Car Averager (BCA 1/2), Hewlett-Packard 8008A Pulse Generator (PG), Hamamatsu C11184 amplifier (AMP 1), Texas Instruments OPA659DRB wideband, unity-gain stable, JFET input operational amplifier (AMP 2), Hamamatsu H11526 - 20-NF photo-multiplier tube (PMT). Labels for QRPS input are not listed because they are not used in the electronic configuration. Each circle shown for the oscilloscope indicates an input channel.

pulse is produced by the Proto-MPEX trigger system $34.15 \mathrm{~s}$ before the plasma pulse, referred to as $t-30$, and is sent to a National Instruments CA-1000 configurable connector accessory enclosure. After a delay of $34.15 \mathrm{~s}$, i.e., $t+4.15$, a square wave pulse is produced by the CA1000 that coincides with the ignition of the plasma and has a width equal to that of the plasma pulse (nominally $1 \mathrm{~s}$ for this work). The generated pulse is transmitted to a custom-built AND gate. Independent of the plasma production pre-pulse, the TALIF laser is always firing. The analog output Q-switch pulse is connected to the other input of the AND gate, with the output connected to a National Instruments DAQ card to monitor triggers for data collection. Only when both the square wave pulse generated during the plasma pulse and the Q-switch output pulse are true will the DAQ card receive a pulse to collect boxcar signal. It is important to 
note that in many cases, similar systems, e.g. Thomson scattering systems, use the pre-plasma pulse to sync the flash lamps so that the laser fires at a well-defined time during the plasma pulse, allowing for time resolved measurements on the scale of the laser repetition rate. The system described here is different because it has a dye stage that is pumped by the traditional YAG laser and, as a result, cannot use this standard method to sync the laser to the pulse; the details surrounding this are discussed later in this section.

Laser wavelength, energy, and linewidth are monitored at the dye stage by the Ångstrom/High Finesse Ws-7 wavelength meter and are simultaneously recorded by the DAQ software. Since the wavelength meter has a digital output and the other primary components of the TALIF electronics are analog output, the wavemeter and data collection routines are simultaneously and independently spawned on the acquisition computer. The measured wavelengths and signal pulses are matched up at a later time by the time stamps recorded with each data point. Fluorescence is collected by a $\varnothing 600 \mu \mathrm{m}$ or $\varnothing 1.5 \mathrm{~mm}$ core multimode fiber and is amplified by a Hamamatsu H11526-20-NF photomultiplier tube (PMT). The PMT has the ability to monitor signal continuously or to be gated on ns timescales.

When applicable, the PMT is gated using a Stanford Research Systems SR250 Gated Integrator and Box Car Averager, hereafter referred to as the BoxCar. While the BoxCar has a multitude of capabilities, it is desirable to use it as a variable gating device due to its ability to finely adjust a gate pulse width down to singles of ns and delay the pulse on similar time scales, making it suitable to gate the PMT. The gate output from the BoxCar is amplified by a Texas Instruments OPA659DRB wideband, unity-gain stable, JFET input operational amplifier which has a typical rise and fall time of $1.3 \mathrm{~ns}$ and a bandwidth of $350 \mathrm{MHz}$. The amplifier also helps to shape the gate pulse sent to the PMT. The BoxCar used for gating is triggered by the flash lamp output pulse from the laser power supply and is delayed in time via the same BoxCar so that the gate pulse turns the PMT on and off only around times at which the laser pulse is present in the plasma.

PMT output is amplified and pulse shaped by a Hamamatsu C11184 amplifier that has a typical rise and fall time of $1.2 \mathrm{~ns}$ and $300 \mathrm{MHz}$ bandwidth. The amplified raw PMT signal is sent to a second BoxCar that is identical to the BoxCar described above. The amplified signal is also monitored by an oscilloscope for real-time visualization of the PMT output. The BoxCar 


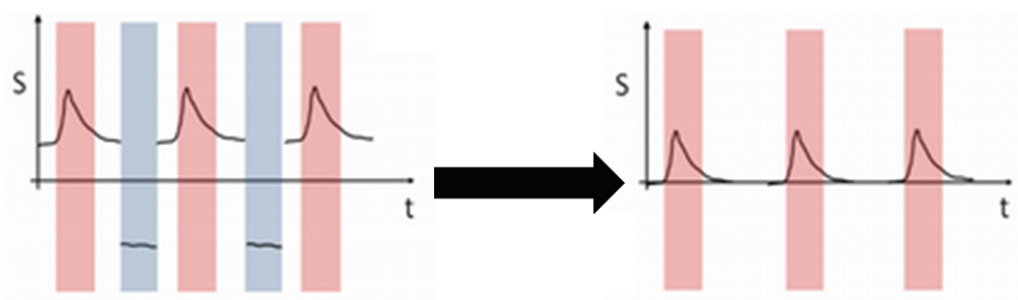

FiguRE 4.16: Schematic of the active background subtraction performed by the BoxCar. A trigger at twice the repetition rate of the laser $(40 \mathrm{~Hz})$ is sent to the BoxCar, one that coincides with the laser pulse in the plasma (red regions) and one when the laser pulse is not present (blue regions). For each trigger the signal under the BoxCar window is integrated. Each laser pulse will have the inverse of the following background integration added to it creating a background subtracted signal. Adapted from Ref. [30]. Reproduced with permission. All rights reserved.

has several optional outputs for data acquisition. The first output used is a direct pass of the PMT signal output. The output is supplied directly to a second channel on an oscilloscope for real-time monitoring of the BoxCar's interpretation of the PMT's output. Monitoring this output from the BoxCar is not a necessity but does rule out a possible electronic malfunction in the event that no signal is collected.

Analyzed signal, i.e., signal recorded by the DAQ system, is a result of active background subtraction from the BoxCar. The active background removal relies on the BoxCar being triggered at twice the frequency of the laser, i.e., $40 \mathrm{~Hz}$. To accomplish this, the Q-switch advance sync from the Quanta Ray power supply is used. The Q-switch advance sync is a variable delay output as referenced from the Q-switch output. This pulse is sent to a HewlettPackard 8008A Pulse Generator that receives a single trigger and creates two output triggers. Approximately $40 \mu \mathrm{s}$ separates the two pulses. It is not a requirement that the double trigger be equally spaced from each other during laser pulses, only that one of the trigger pulses is during a time that no laser emission is present in the plasma. The output of the double pulse generator is sent to the trigger input on the BoxCar. For each pulse received by the BoxCar, the data located inside the BoxCar window is integrated. Consecutive pulses are subtracted from each other to obtain a background subtracted integrated signal. ${ }^{3}$ Figure 4.16 depicts the background subtraction process performed by the BoxCar. The background subtracted signal is then sent to the DAQ card to be recorded as TALIF signal.

\footnotetext{
${ }^{3}$ Actually, inverse of the second signal integration is added to the first, providing the same result as if the two signals were subtracted.
} 
As mentioned above, the TALIF laser system cannot be synced with the plasma pulse like other systems. Since the gain of the dye has a vastly different stabilization time than the lasing mechanisms of the pump laser, it takes much longer for the UV energy-per-pulse to reach a stable maximum. If the firing of the laser were to be synced to Proto-MPEX rf discharge pulses, the laser would essentially remain idle (flash lamps flashing only) for the duty cycle of Proto-MPEX. The duty cycle of Proto-MPEX is nominally 3 minutes, but can be greater than 10 minutes in some cases. The time it takes for the dye laser to produce a maximum stable energy output, also known as the rise time, is longer than the maximum Proto-MPEX pre-pulse. Figure 4.17 shows the time for the TALIF laser system to reach $90 \%$ maximum energy-per-pulse for a "break-time" of (a) 3, (b) 5, and (c) 10 minutes. For the best-case scenario, the laser rise time is nearly triple the maximum pre-pulse time supplied from Proto-MPEX. The rise time of laser energy was measured with a Gentec E-Maestro energy meter. Therefore, the laser is also firing, independent of whether the laser pulse coincide with the plasma pulse or not.

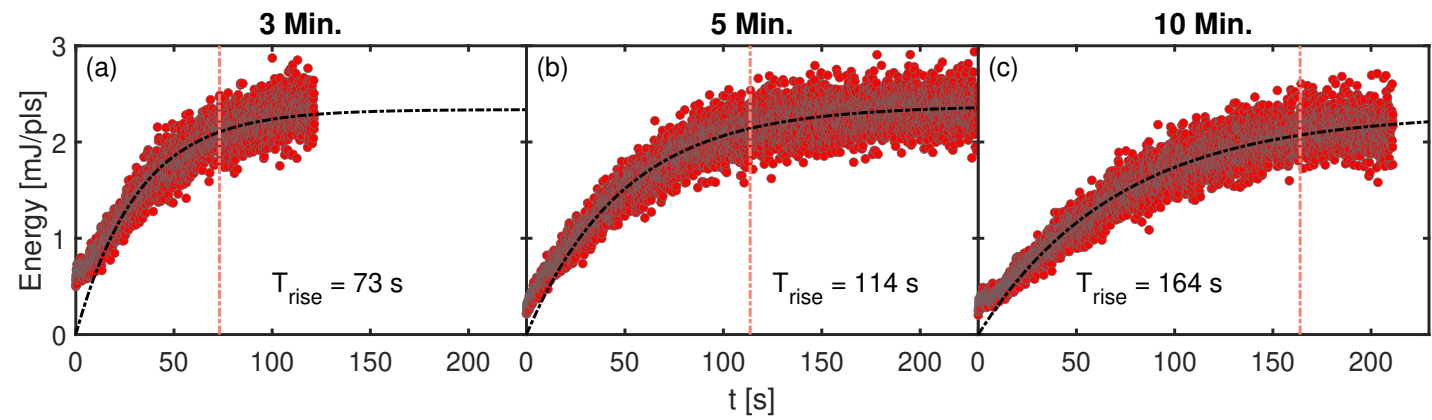

FigURE 4.17: Laser energy-per-pulse rise time for three cases: (a) 3 minutes of interrupted Q-switching, (b) 5 minutes of interrupted Q-switching, and (c) 10 minutes of interrupted Q-switching. Raw energy-per-pulse data are depicted by the red circles, a fit characterizing the rise time is shown by the black dot-dash line, and the time for the energy to reach $90 \%$ of the maximum value is shown by the pink dot-dash line. For all cases, the flash lamps were flashing during the Q-switch downtime. Even for optimal repetition rates of Proto-MPEX (3 minutes), the rise time is too great to use the Proto-MPEX pre-pulse to sync the laser flash lamps.

The DAQ layout is slightly modified for calibration TALIF measurements. Figure 4.18 shows the layout for these measurements. The main difference is that the system no longer needs a pre-pulse from Proto-MPEX. Since the chamber is backfilled to a static pressure, every pulse is collected. In this case, the AND gate is bypassed and there is no longer a need to gate the PMT. Instead, the Q-switch output from the laser power supply is connected directly to the PF1 channel on the National Instruments DAQ card and pulses are measured consecutively. 


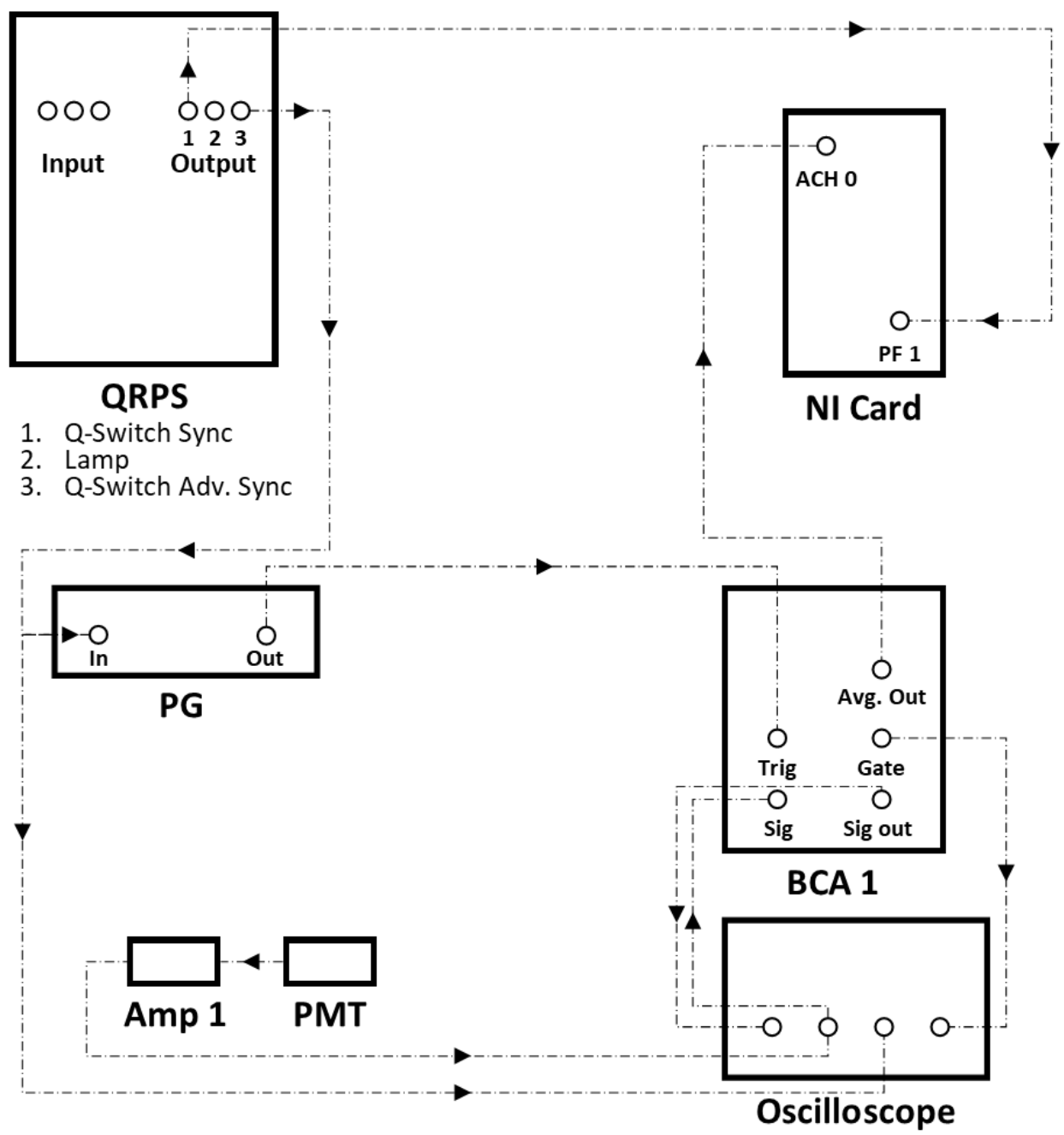

FIGURE 4.18: TALIF electronics box diagram of calibration (xenon and krypton) measurements (described in text). Abbreviations: Quanta Ray power supply (QRPS), National Instruments BNC-2110 BNC adapter DAQ card (NI Card), Stanford Research Systems SR250 Gated Integrator and Box Car Averager (BCA 1), Hewlett-Packard 8008A Pulse Generator (PG), Hamamatsu C11184 amplifier (AMP 1), Hamamatsu H11526 - 20-NF photo-multiplier tube (PMT). Labels for QRPS input are not listed because they are not used in the electronic configuration. Each circle shown for the oscilloscope indicates an input channel.

Since the TALIF laser system pulse length is on ns time scales, the electronics described above need to have time resolution capable of collecting only TALIF signal. Additionally, the detector needs to be sensitive to measuring the fluorescence of each scheme and should provide apt amplification of measured signal. The PMT used in this work offers a large range of amplified gain for a given applied PMT voltage. Figure 4.19 shows the PMT gain as a function of applied voltage. This figure was reproduced from measured data supplied by Hamamatsu. The gain range from activation voltage to maximum applied voltage spans nearly three decades (high $10^{3} \times$ gain to nearly $10^{7} \times$ gain). The PMT used needs to be sensitive to all fluorescence wavelengths 


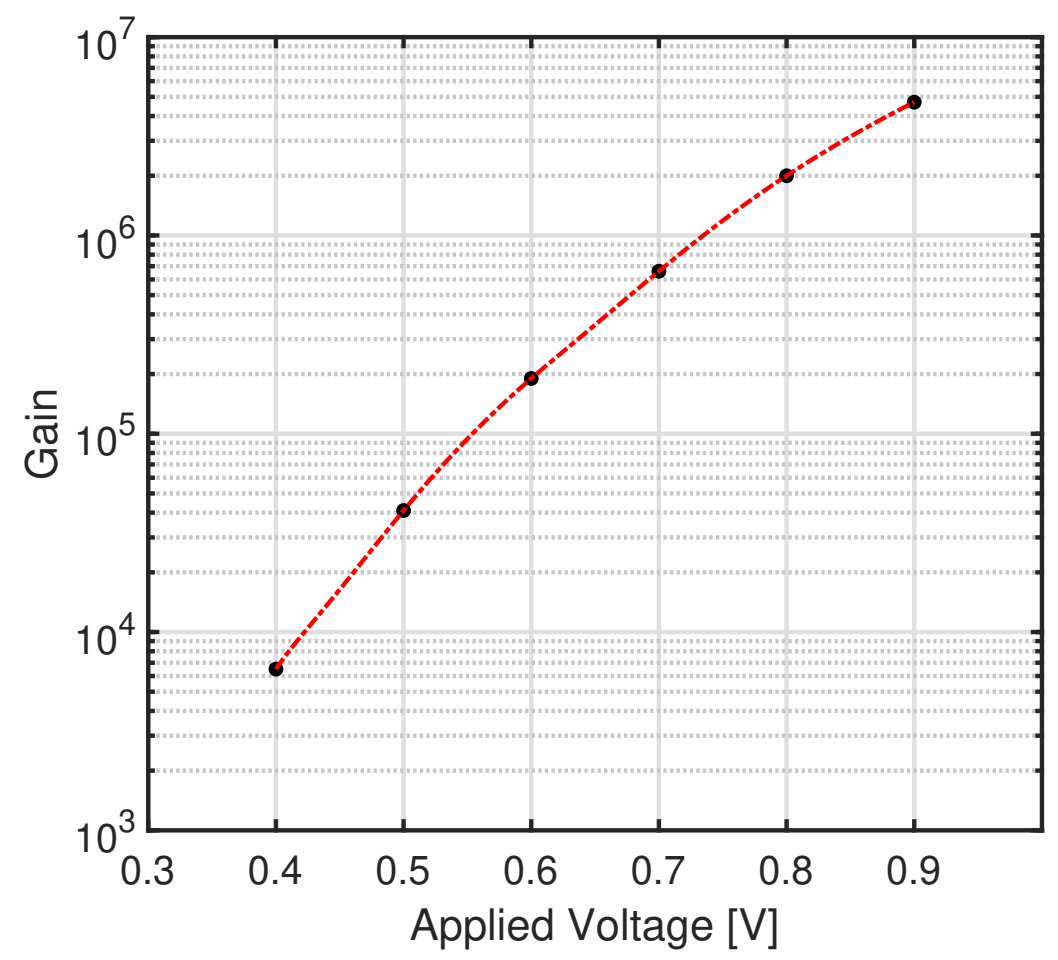

Figure 4.19: PMT gain for a given applied voltage. Though applied voltages less than $0.4 \mathrm{~V}$ can be applied, the suggested "activation" voltage for this PMT is nominally around $0.4 \mathrm{~V}$. Note that an internal amplifier increases the applied voltage to the PMT by a factor of 1000 . Gain data courtesy of Hamamatsu Photonics K.K.

of the TALIF schemes used in this work (i.e. $\sim 650 \mathrm{~nm}$ to $\sim 830 \mathrm{~nm}$ ). Figure 4.20 shows the quantum efficiency $Q E$ as a function of wavelength. Hamamatsu does not directly supply the $Q E$ of this detector. Instead a quantity called cathode radiant sensitivity $S$ is supplied. The cathode radiant sensitivity is a quantity that describes the photoelectric current from the detector cathode divided by the radiant power as a function of wavelength. The relation between quantum efficiency (number of incident photons converted to electrons) and cathode radiant sensitivity is

$$
Q E=\left(\frac{S \cdot 1240}{\lambda}\right)
$$

where $Q E$ is the quantum efficiency, $S$ is the cathode radiant sensitivity, and $\lambda$ in $\mathrm{nm}$ is the wavelength of the incident photon. 


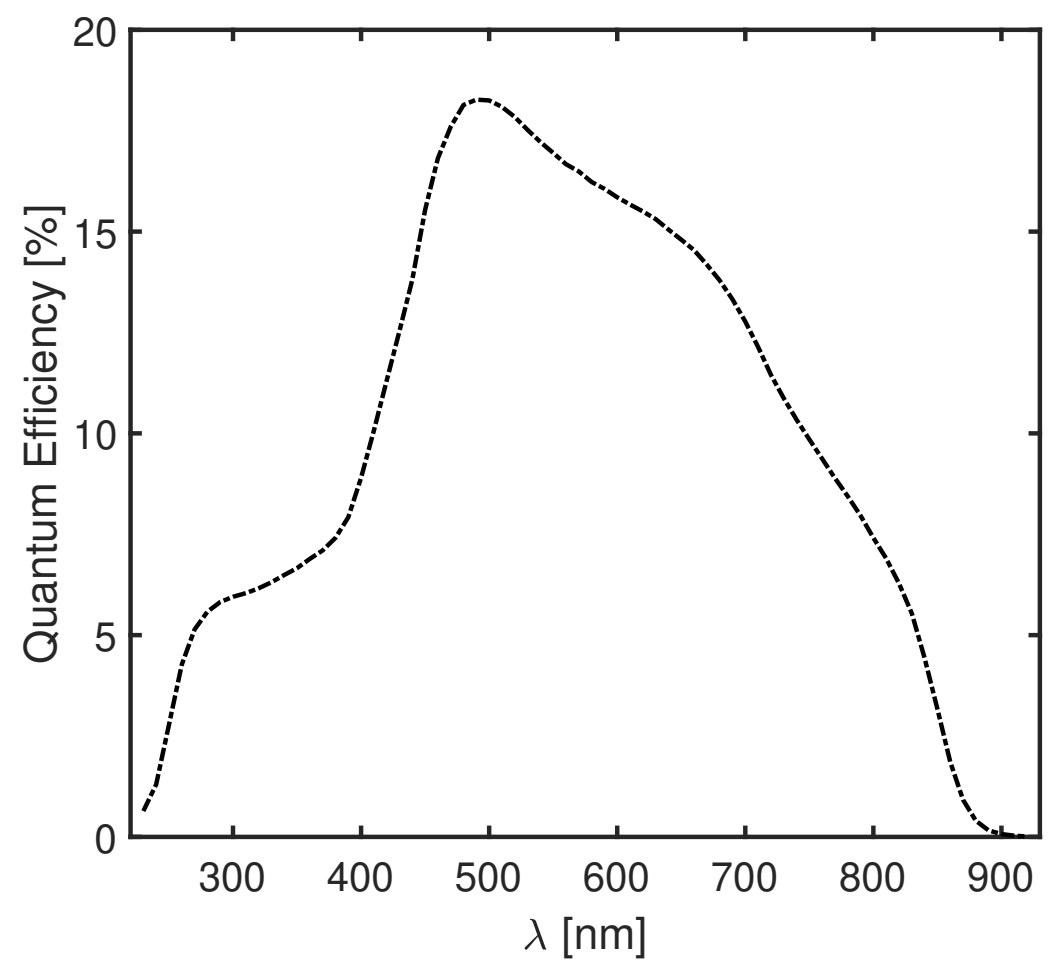

Figure 4.20: Quantum efficiency of the Hamamatsu H11526 - 20-NF PMT. Cathode Radiant Sensitivity data courtesy of Hamamatsu Photonics K.K.

Raw data supplied by Hamamatsu for Figs. 4.19 and 4.20 are provided in App. E.

\subsubsection{Prototype Material-Plasma Exposure eXperiment (Proto-MPEX)}

It is becoming evermore important to understand how plasmas interact with materials. As fusion devices progressively come closer to creating sustainable energy, it is obvious that the integrity of the plasma chamber is a leading concern. For example, PFCs in the divertor region of the International Thermonuclear Experimental Reactor (ITER) design have been identified as a crucial element for the success and longevity of this fusion tokamak [101]. However, it is rarely practical to investigate plasma-material interactions in a device such as ITER (or any tokamak) due to the large overhead of operating these devices. For this reason, Oak Ridge National Laboratory (ORNL) created a device that can study PFCs in fusion-relevant plasmas more efficiently. Linear plasma devices provide a simple geometry in which PMI experiments can be conducted [102]. Specifically, helicon sources provide excellent test beds for high density $\left(>10^{19} \mathrm{~m}^{-3}\right)$ "hot" plasmas [103]. While the geometry and physics of a linear helicon device provide a clear path to studying PFCs in simulated divertor scenarios, it is still a simplified 
geometry and cannot account for the complex configurations of future divertor systems [66]. However, the simplified approach offered by linear devices still provides bulk information for PFC studies and there are many linear devices created to study unique aspects of divertor physics [104]. For these reasons, the Material Plasma Exposure eXperiment (MPEX) [102], a linear helicon plasma device, has been under iterative designs to produce fusion-relevant plasmas to study PFC and SOL physics.

A final design of a steady-state MPEX is nearing completion [102, 105-107], but a scaled down prototype device was constructed at ORNL for proof-of-principle measurements. Proto-MPEX $[27,105,108]$ is a pulsed linear helicon device designed and built at ORNL that, over the course of its existence, has operated in a relevant parameter space for PFC experiments, as well as paved the way for the construction of MPEX [103].

The layout of Proto-MPEX will be described in regards to its configuration during the measurements for this work. Figure 4.21 shows a side view of Proto-MPEX. Proto-MPEX can produce

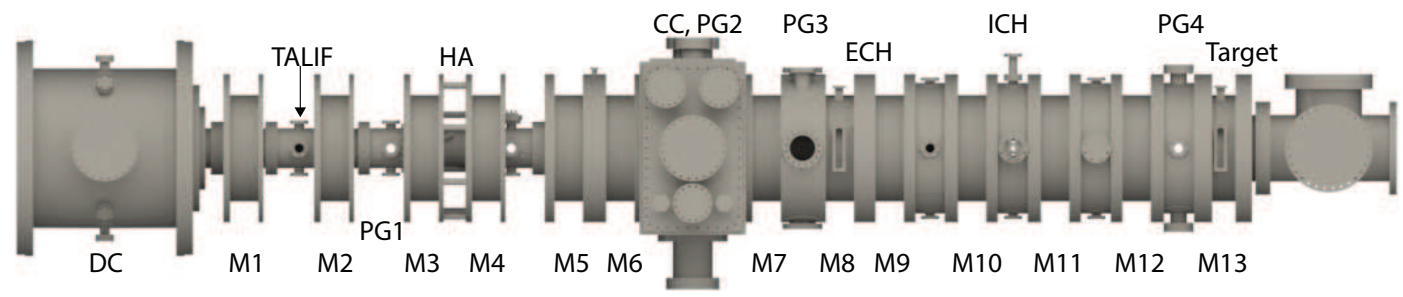

Figure 4.21: Rendered image of Proto-MPEX. Abbreviations: Dump chamber (DC), magnets $1-13$ (M1-M13), TALIF measurement location (TALIF), pressure gauges $1-4$ (PG1-PG4), helicon antenna (HA), electron cyclotron auxiliary heating location (ECH), ion cyclotron auxiliary heating location (ICH), and location of target plate (Target).

up to $330 \mathrm{~kW}$ of power (installed) ${ }^{4}$ and consists of 13 water-cooled copper solenoid electromagnets; a double helix, half-turn, azimuthal mode $+1(m=+1)$, water-cooled, copper antenna ${ }^{5}$ (HA); "downstream" (right of helicon antenna in Fig. 4.21) and "upstream" (left of antenna in Fig. 4.21) target plates; electron cyclotron heating (ECH) injection; and an ion cyclotron heating (ICH) copper antenna.

The 13 electromagnets have an inner diameter of $\sim 22 \mathrm{~cm}$ with 40 turns each. Subsets of magnets are controlled independently to create a user-defined magnetic field profile over a range of $0-2 \mathrm{~T}$ (profile described below). The solenoids that are currently a part of Proto-MPEX

\footnotetext{
${ }^{4}$ Current upgrades have increased the installed power to $\sim 430 \mathrm{~kW}$ of power, though typical injected powers remain much lower than the installed number [107]

${ }^{5}$ An azimuthal mode +1 helicon antenna indicates a global right hand rotation pattern of the wave pattern.
} 
were repurposed from the ELMO Bumpy Torus (EBT) [109] experiment and are designed to run at a constant current of 9,000 A. No single magnet was driven at a current higher than $\sim 4500$ A for this experiment. A large magnetic field, i.e, $>0.5 \mathrm{~T}$, is produced by M1, which then decreases considerably from M2 to M5 ( $0.05 \mathrm{~T})$ before increasing back to a high magnetic field at M6. A considerable dip in magnetic field strength occurs between M6 and M7 and then increases to magnetic field strengths of $\sim 1 \mathrm{~T}$ for the remainder of M7 through M13. Figure 4.22 shows the magnetic field profile for the measurements in this work. The magnetic field configuration is typically unique for different experiments to optimize performance. That is, the magnetic field shape is different for ECH optimized experiments, ICH optimized experiments, and overall maximum heat flux experiments. The magnetic field configuration shown in Fig. 4.22 is commonly employed for ICH investigations, though ICH auxiliary heating was not used for these measurements. The rectangles indicate the magnet locations and are labeled as they are referred to in the text. The red dot-dash line shows the axial location of TALIF measurements.

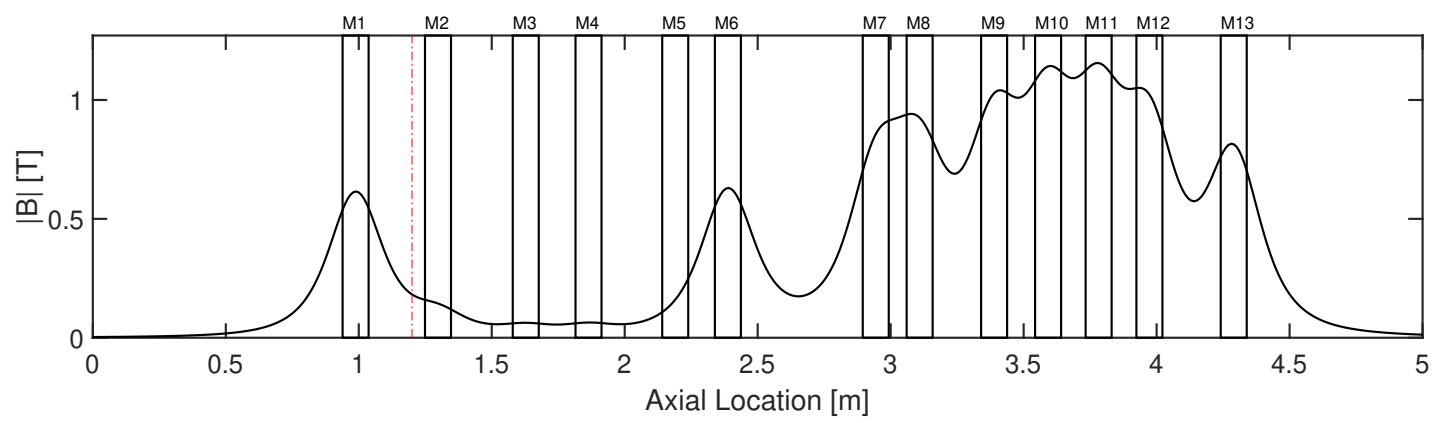

FiguRE 4.22: Magnetic field profile for TALIF measurements presented here. Black rectangles indicate magnet locations and are labeled as they are referred to in the text. The red dot-dash line marks the TALIF measurement location.

The magnet locations are referred to by the order they are situated in the layout of the device, i.e., M1 through M13. Optical access locations between the magnets are referenced to the lower magnet number with the addition of " 0.5 ". For example, the port access between magnets M1 and M2 is port 1.5. It is important to note that the port numbers do not indicate a linear distance in any physical units, i.e., port 1.5 does not correspond to $1.5 \mathrm{~m}$ along the chamber axis.

The vacuum vessel begins with a stainless steel target dump chamber. The dump tank is coupled to a series of $15.2 \mathrm{~cm}$ diameter stainless steel chamber components that total approximately 
$1.5 \mathrm{~m}$ in length (roughly the distance from M1 to M5). Along this path are several $23 / 4$ " diagnostic access ports for the various optical and mechanical needs of Proto-MPEX. M6 and M7 are coupled to a rectangular aluminum central chamber equipped with several viewing ports and diagnostic access flanges. M5 through M9 are part of the vacuum boundary with several viewing ports for diagnostic access. M10 and M11 are ex-vacuum magnets and M12 and M13 are part of the vacuum boundary again. An additional ECH injection and a custom diagnostic flange that facilitates ample diagnostic access to the downstream target are also located in this section of the vacuum vessel.

A total of 4 turbo-molecular drag pumps are used to maintain a base pressure of $\sim 10^{-6}$ Torr. From left to right in Fig. 4.21: a $150 \mathrm{l} / \mathrm{s}$ turbo pump is attached to the dump tank; two larger turbo pumps are located at the central chamber that have a pumping capacity of $2800 \mathrm{l} / \mathrm{s}$ and 1000 l/s, respectively; and a final pump is located beyond the downstream target plate with a pumping rate of $2500 \mathrm{l} / \mathrm{s}$. The downstream target plate is attached to a retractable motorized stage that allows for the complete removal of the target plate from the main vacuum vessel. At the interface of the retractable stage and the Proto-MPEX chamber is a pneumatic gate valve that provides the option for the target plate to be completely isolated from Proto-MPEX. The advantage of this feature is the ability to remove the target plate for analysis without breaking vacuum of the main chamber. A small turbo pump is attached to the target plate removal vacuum components for evacuation of the target stage during re-insertion. This turbo is not needed to maintain the base pressure of the machine and is an optional additional pumping source that has negligible effect on the base pressure of Proto-MPEX.

Pressure is monitored at several locations along Proto-MPEX. An MKS Instruments 627 Baratron pressure gauge is placed at ports 2.5, 6.5, 8.5 and 12.5 (as indicated in Fig. 4.21). Gas can be injected at ports 1.5, 2.5, and 4.5. Gas is injected through a nozzle at port 1.5 and a gas ring along the inner diameter of the chamber at 2.5 and 4.5. Gas flows of 10 standard liters-per-minute (SLM) are achievable with MKS Instruments mass flow controllers. For this work, gas flows greater than normal were used, i.e., gas flows greater than 2.5 SLM injected at ports 1.5 and 2.5. Obstruction plates that restrict flow, referred to as "skimmers," are placed in various locations of Proto-MPEX to create differential pumping, which results in a non-uniform 
pressure profile. To this end, the pressure gauge closest to the TALIF injection (port 2.5) is used for the most accurate estimation of the local neutral pressure for TALIF measurements.

The plasma source uses a double helix, half-turn, $m=+1$, water-cooled, copper antenna located between magnets M3 and M4. Radio frequencies (rf) of $9-21 \mathrm{MHz}$ are applied to generate the plasma. The copper antenna surrounds an aluminum nitride (Ceradyne Cerally ${ }^{\circledR} 1370$ DP) "window" that is transparent to rf. For this work, $13.56 \mathrm{MHz}$ rf was used. At the time of these measurements, a maximum of $\sim 120 \mathrm{~kW}$ of forward power from a Continental Electronics FRT86 Power Supply could be applied ${ }^{6}$, though measurements for deuterium TALIF were conducted at much smaller forward powers.

Additional heating mechanisms (ECH, ICH) add to the installed power of Proto-MPEX. At the time of these experiments, $200 \mathrm{~kW}$ of power from a Varian VGA-800 power supply could be applied for ECH. A frequency of $28 \mathrm{GHz}$ was necessary for auxiliary electron heating at the central chamber. Microwaves are injected at an angle of $20^{\circ}$ to the face of the central chamber wall for most efficient heating through electron Bernstein wave (EBW) coupling [27, 110]. Currently, Proto-MPEX has added the capability to inject microwaves at spool 8.5 for ECH with a frequency of $105 \mathrm{GHz}$ [103]. ECH was not used as an auxiliary heating mechanism for any measurements in this work. A second half-turn, $m=+1$, copper antenna is inserted into the chamber between magnets M10 and M11 for ICH. The helical twist for this antenna is opposite the source antenna. While the antenna is inserted into the vacuum vessel, it is separated from the plasma by a quartz tube that acts to extend the longevity of the ICH antenna. The ICH antenna is typically operated at $8.5 \mathrm{MHz}$ at a forward power of $\sim 30 \mathrm{~kW}$ using an FRT-85 power supply. For the most recent results and expected upgrades for Proto-MPEX at the time of this document, the reader is directed to Ref. [103].

Proto-MPEX utilizes numerous diagnostics to characterize the helicon plasma. A full list of diagnostics used on Proto-MPEX is shown in Table 4.2. Detailed descriptions of each diagnostic are outside the scope of this work and are only listed here. The motivated reader is directed to the citations (where available) listed in Table 4.2.

\footnotetext{
${ }^{6}$ Currently, Proto-MPEX can routinely source rf powers greater than $120 \mathrm{~kW}$ with the addition of a second power supply [103]. However, operation is restricted to $13.56 \mathrm{MHz}$.
} 


\begin{tabular}{|c|c|c|c|}
\hline Diagnostic & Results & Variable & References \\
\hline AXUV diodes & Power dissipated from radiation & $P_{r a d}$ & {$[28,111,112]$} \\
\hline B-dot probe $(\dot{\mathrm{B}})$ & Time varying magnetic field & $\dot{\mathrm{B}}$ & {$[28,113,114]$} \\
\hline Baratron pressure gauges & Neutral pressure & $p_{n}$ & [115] \\
\hline Double Langmuir probes & $\begin{array}{l}\text { Electron temperature } \\
\text { Electron density }\end{array}$ & $\begin{array}{l}T_{e} \\
n_{e}\end{array}$ & {$[28,116]$} \\
\hline Fast-camera imaging & Plasma emission dynamics & - & \\
\hline Fluoroptic probes & $\begin{array}{l}\text { Thermal temperature } \\
\text { of helicon window }\end{array}$ & $T_{\text {helicon }}$ & {$[28,117]$} \\
\hline HELIOS & $\begin{array}{l}\text { Electron temperature } \\
\text { Electron density }\end{array}$ & $\begin{array}{l}T_{e} \\
n_{e}\end{array}$ & {$[28,118]$} \\
\hline $\begin{array}{l}\text { High resolution } \\
\text { McPherson spectrometer }\end{array}$ & $\begin{array}{l}\text { Ion temperature } \\
\text { Rotational velocity } \\
\text { Axial velocity }\end{array}$ & $\begin{array}{c}T_{i} \\
v_{\text {rot }} \\
v_{z} \\
\end{array}$ & \\
\hline Infrared thermography & $\begin{array}{l}\text { Target surface temperature } \\
\text { Target heat flux }\end{array}$ & $\begin{array}{c}T_{\text {surf,target }} \\
q_{\text {target }}\end{array}$ & {$[28,113,119]$} \\
\hline Mach-double probe & $\begin{array}{l}\text { Mach number } \\
\text { Electron temperature } \\
\text { Electron density }\end{array}$ & $\begin{array}{l}\mathrm{M} \\
T_{e} \\
n_{e}\end{array}$ & {$[66,116]$} \\
\hline Mach probes & Mach number & $\mathrm{M}$ & {$[28,65,66,116]$} \\
\hline Metal foil bolometers & Power dissipated from radiation & $P_{\text {rad }}$ & {$[28,120-123]$} \\
\hline Microwave interferometer & Electron density & $n_{e}$ & {$[124,125]$} \\
\hline Residual gas analyzer (RGA) & Partial neutral pressures & $p_{n, z}$ & {$[126]$} \\
\hline Retarding field analyzer & Ion energy & $\overline{E_{i}}$ & {$[28,127]$} \\
\hline Sniffer probe & Microwave absorption & - & \\
\hline Survey spectrometer & Species density & $n_{z}$ & \\
\hline SXR diode & Power dissipated from radiation & $P_{\text {rad }}$ & [28] \\
\hline TALIF & $\begin{array}{l}\text { Neutral temperature } \\
\text { Neutral bulk flow } \\
\text { Absolute neutral density }\end{array}$ & $\begin{array}{c}T_{n} \\
v_{n, \circ} \\
n_{\circ}\end{array}$ & {$[30,96]^{7}$} \\
\hline Target Langmuir probes & $\begin{array}{l}\text { Electron temperature } \\
\text { Electron density } \\
\text { Ion flux }\end{array}$ & $\begin{array}{l}T_{e} \\
n_{e} \\
\Gamma_{i} \\
\end{array}$ & {$[6,28,116]$} \\
\hline Thermocouples & Bulk target temperature & $T_{\text {bulk,target }}$ & {$[28,119]$} \\
\hline Thomson scattering & $\begin{array}{l}\text { Electron temperature } \\
\text { Electron density }\end{array}$ & $\begin{array}{l}T_{e} \\
n_{e}\end{array}$ & {$[46,110,128]$} \\
\hline
\end{tabular}

TABle 4.2: List of diagnostics used on Proto-MPEX and the quantities measured with each diagnostic. Abbreviations: absolute extended ultra violet (AXUV), soft X-ray (SXR).

\footnotetext{
${ }^{7}$ Discussed in detail in Ch. 2
} 


\section{Chapter 5}

\section{Results: Iodine LIF}

Singly ionized atomic iodine LIF measurements were made using an Evenson microwave cavity and a sealed Opthos Inc. reference cell containing solid iodine to develop the tools needed to measure I II flows when iodine is used as a propellant for electric propulsion. In this chapter, I II VDFs are presented in the absence of any external magnetic field and for a variety of magnetic field strengths. The hyperfine splitting of the probed transition is significant. Monte-Carlo fitting routines were used to provide a "most-probable" temperature in the case of significant hyperfine splitting. Lastly, hyperfine splitting combined with Zeeman splitting creates an extremely complex spectrum, and while the individual transitions are not resolved, $\sigma$ and $\pi$ groups are resolved for strong magnetic fields and these measurements suggest a method for determining the background magnetic field from the $\sigma$-peak separation.

\subsection{II Passive Spectroscopy}

Initial investigations of the iodine pump transition and fluorescence were conducted using passive spectroscopy. Measurements confirmed the findings of Hargus et al., showing that pump state population is inversely proportional to chilling finger temperature $T_{\mathrm{CF}}$. For this reason, measurements were taken at chilling finger temperatures much less than room temperature, i.e., < 5 C. Figure 5.1 shows pump transition intensity measured with a 1.33 m Czerny-Turner monochromator as function of pressure (chilling finger temperature) for a range of forward microwave powers. Figure 5.2 shows an example spectrum for both the (a) pump and (b) fluorescent transitions. Spectra were obtained at a power of $30 \mathrm{~W}$ and chilling finger temperature 


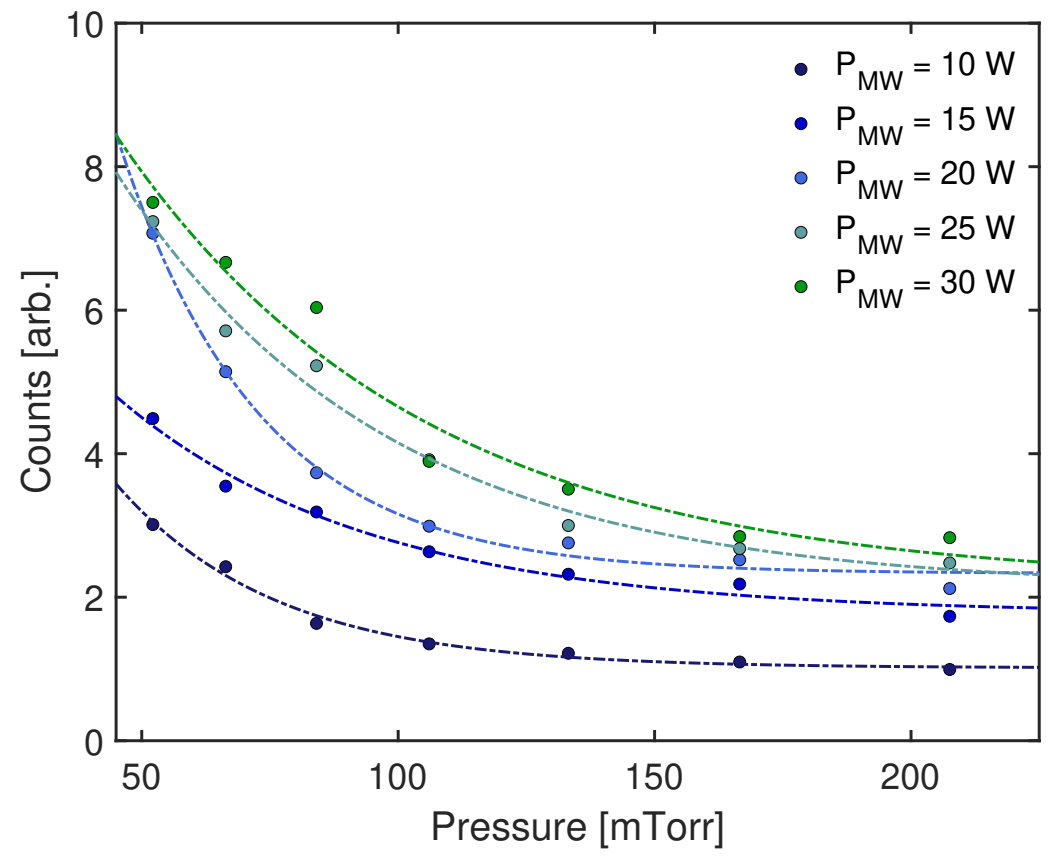

FIGURE 5.1: Pump transition intensity as a function of cell iodine partial pressure for microwave powers ranging from $10 \mathrm{~W}-30 \mathrm{~W}$. State population is proportional to microwave power and inversely proportional to pressure (chilling finger temperature). Trends are in good agreement with measurements reported in Ref. [22].

of $5 \mathrm{C}$ ( $\sim 52 \mathrm{mTorr})$. While Fig. 5.2 shows a transition is remarkably close to the target transition, the transition is not within the scanning range for LIF measurements as shown in the inset figure. Observations also indicate that the minimal transition "pollution", i.e., transitions that

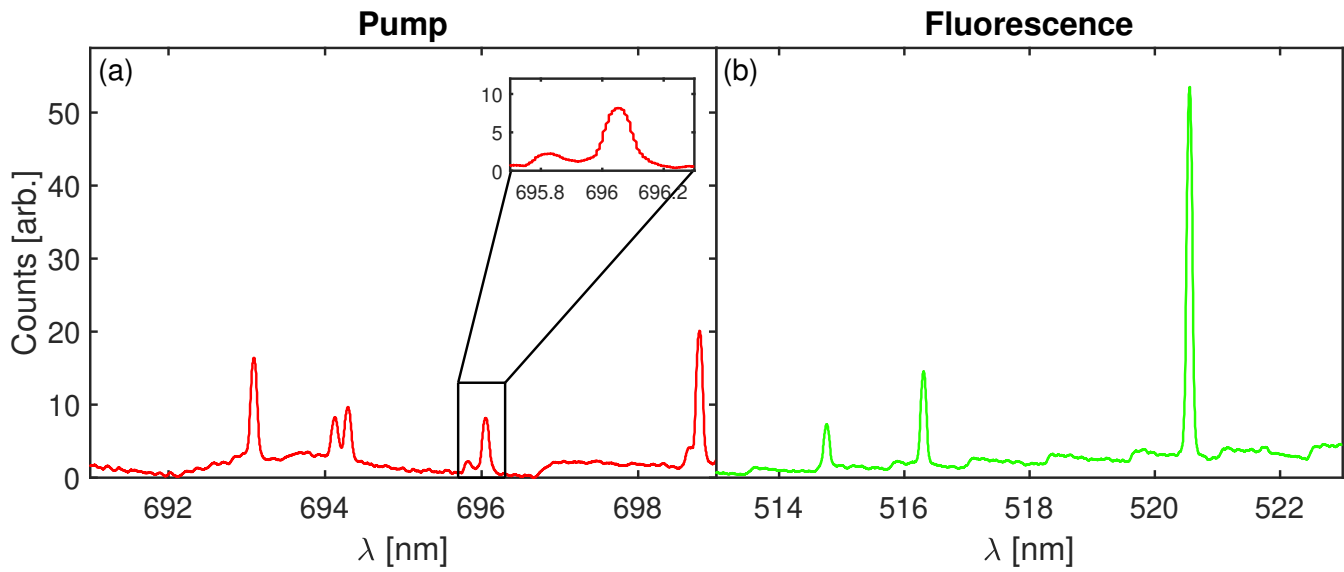

FiguRE 5.2: Spectra emission around the pump transition $(\sim 696 \mathrm{~nm})$ and fluorescence $(\sim 516 \mathrm{~nm})$. Minimal transition "pollution" is present within the scanning range of this transition. Spontaneous emission from surrounding transitions in the collection window (b) is filtered using a lock-in amplifier.

are not part of the proposed LIF scheme, are present at both pump and fluorescent wavelengths. 


\subsection{II LIF}

As described previously, the predicted hyperfine structure of the absorption transition is extensive. Figure 5.3 shows a typical I II ion velocity distribution function (IVDF) measurement. The measurement shows non-Maxwellian lineshape features. The irregular lineshape is caused by hyperfine of the two states probed in this work. A naive single Gaussian fit to the I II lineshape would yield an ion temperature of $\gtrsim 1 \mathrm{eV}$, which is completely inconsistent with the experimental conditions.

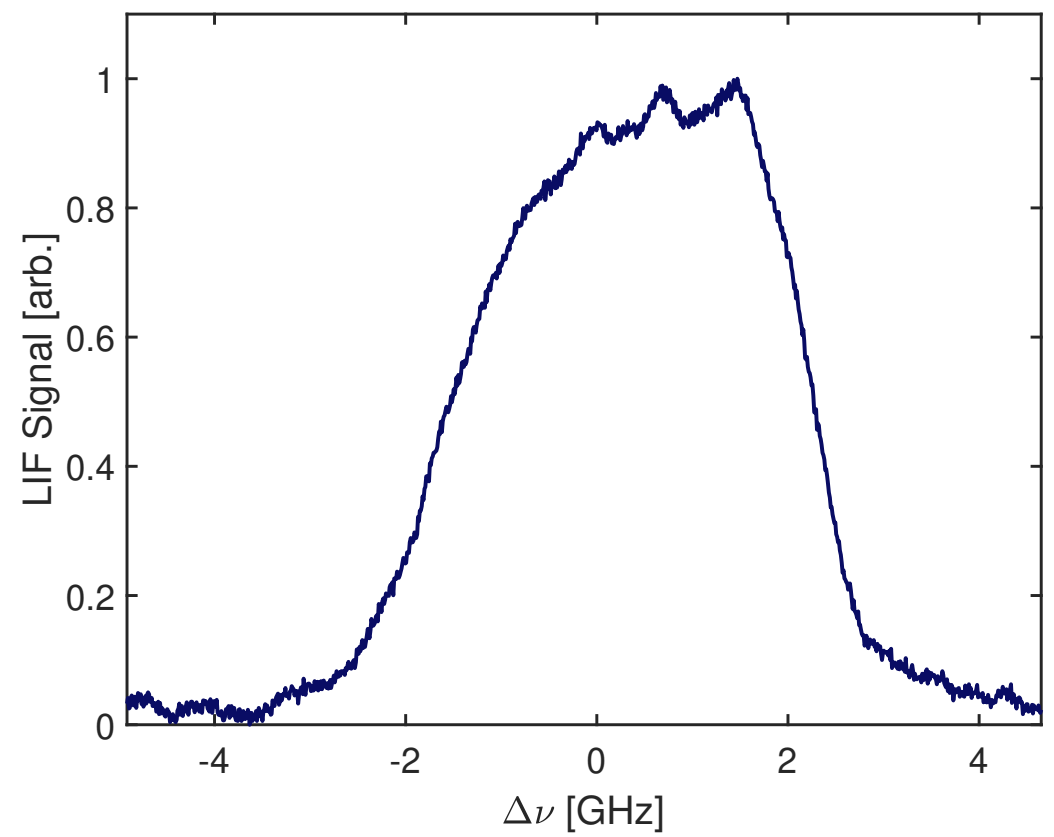

Figure 5.3: Example measured lineshape of I II. Profile is exceptionally broad from hyperfine structure. Fifteen hyperfine transitions are contained within the measured lineshape.

LIF measurements of the I II spectral lineshape confirms results from OES. Figure 5.4 shows consecutive LIF measurements as the pressure (chilling finger temperature) within the cell is increased. Overall SNR increases as pressure (chilling finger temperature) decreases and the emergence of hyperfine structure is seen for pressures below $\sim 85$ mTorr. Decreased signal for higher pressures suggests a state quenching factor that is proportional to pressure for this transition. Since best signal is observed for lower pressures, ${ }^{1}$ all LIF measurements were conducted at $p_{\text {gas }} \leq 52$ mTorr $\left(T_{\mathrm{CF}} \leq 5^{\circ} \mathrm{C}\right)$.

\footnotetext{
${ }^{1} \mathrm{SNR}$ levels off for chilling finger temperatures less than $-10^{\circ} \mathrm{C}$ and plasma is difficult to maintain for temperatures less than $-15^{\circ} \mathrm{C}$.
} 


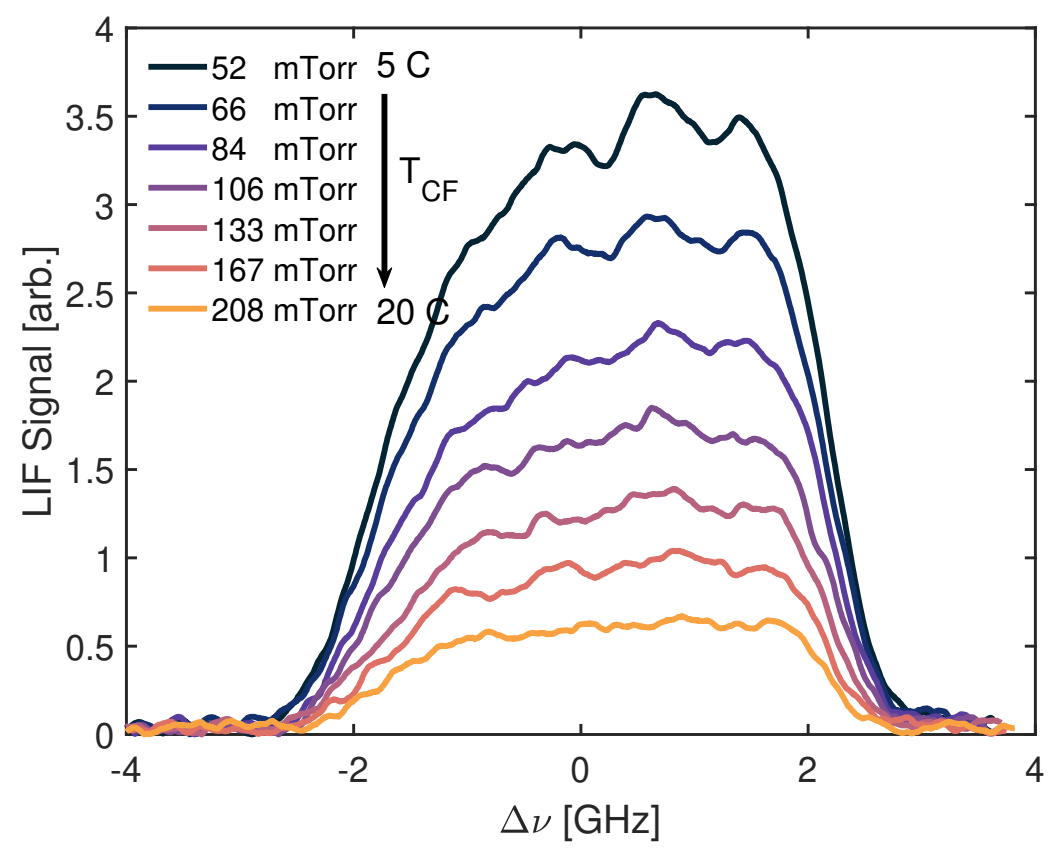

Figure 5.4: LIF collected signal for different gas pressures. Results are in good agreement with initial passive spectroscopy observations. As pressure (chilling finger temperature) decreases, clear emergence of hyperfine structure is observed. Individual hyperfine peaks are dependent on the magnetic dipole and electric quadrupole coupling coefficients. Reproduced from Ref. [20]; reprinted by permission of the American Institute of Aeronautics and Astronautics, Inc.

The analysis presented in Sec. 3.5 predicts hyperfine splitting transitions and clear hyperfine structure and peaks emerge for lower pressures. The hyperfine splitting depends on the coupling coefficient associated with the magnetic dipole moment $A$ and the coupling coefficient associated with the electric quadrupole moment $B$. Determination of these coefficients from these measurements is not possible since individual transitions cannot be resolved from the Doppler-broadened lineshape and the peaks that are observed could be composed of several superimposed hyperfine transitions.

The intensity analysis from Russel-Saunders coupling performed by Hargus et al. predicts that 11 of the 15 transitions dominate the lineshape. Therefore, all fitting routines described here neglect the four weakest transitions. The measured lineshape was fit to

$$
\mathcal{F}=a+\sum_{i=1}^{11} c_{i} \exp \left(-\alpha \frac{\left[f-\left\{f_{\circ}+G_{i}\left(A_{u}, A_{l}, B_{u}, B_{l}\right)\right\}\right]^{2}}{T}\right),
$$

where $a$ is a DC offset, $c_{i}$ is the amplitude of the $i$ 'th hyperfine peak, $\alpha=m \lambda_{\circ} / 2, f_{\circ}$ and $\lambda_{\circ}$ are the rest frequency and wavelength, respectively, and $G_{i}\left(A_{u}, A_{l}, B_{u}, B_{l}\right)$ is a function that restricts the frequency shift from one transition to the next, that is consistent with allowed 
transitions, and depends only on the magnetic dipole and electronic quadrupole coupling coefficients for the upper (subscript $u$ ) and lower (subscript $l$ ) states. Since the goodness of any one fit is extremely sensitive to initial values of the free parameters, a Monte-Carlo style approach of fitting a measured lineshape 10,000 times with Eq. 5.1 with randomized starting parameters was used to identify the most probable values for the the free parameters. Although there exists a prediction for relative intensities of the transitions, the relative amplitudes were left as a free parameter, but the frequencies for each hyperfine peak were constrained to match the hyperfine selection rules and Eq. 3.19. Each transition was forced to have the same ion temperature.

Not all 10,000 trials were used since not all converged. A root-mean-square error (RMSE) of 0.01 was used as the cutoff for an "excellent" fit [129]. RMSE values of 0.05 and 0.08 were defined as "good" and "mediocre" fits, respectively. Of the 10,000 fits, only 794 met the criterion for an excellent fit, while 9,784 met the criterion for being good fits, and 9,914 trials met the criterion for being mediocre fits. From the 794 excellent fits, a "most probable" species temperature was determined to be $0.09 \pm 0.01 \mathrm{eV}$. The estimated iodine ion temperature is comparable to $\mathrm{Xe}$ II ion temperatures measured in similar cells [130]. An attempt was also made to determine the values for the coupling coefficients using this fitting method. However, the magnetic dipole values are only weakly constrained with this method and the values for the electric quadrupole coupling coefficients seem to have little effect on the goodness-of-fit [20]. Optimal fits were also determined using the r-squared value as an indicator of goodness-of-fit. A r-squared value of 0.9995 was chosen as the threshold for an excellent fit. Beginning with 10,000, a total of 558 fits met the r-squared criterion suggesting a most probable temperature of $0.08 \pm 0.01 \mathrm{eV}$. For completeness, all values determined from this fit method are presented in Tab. 5.1 (i.e. excellent, good, and mediocre).

The r-squared based analysis also only weakly constrains the allowed values for the coupling coefficients. Figure 5.5 shows an example fit deemed as the "best" fit (having the lowest RMSE value). Eleven peaks are shown and it should be noted that this is only one possible configuration of the hyperfine structure and should not be considered the actual verified hyperfine structure of this transition. The same fit trial yielded the best fit as determined by both the r-squared value and the RMSE. Therefore, Fig. 5.5 should be viewed as the best overall fit. 


\begin{tabular}{lcccc}
\hline \hline & $\begin{array}{c}\text { Excellent } \\
(\mathrm{RMSE} \leq 0.01)\end{array}$ & $\begin{array}{c}\text { Good } \\
(\mathrm{RMSE} \leq 0.05)\end{array}$ & $\begin{array}{c}\text { Mediocre } \\
(\mathrm{RMSE} \leq 0.08)\end{array}$ & $\begin{array}{c}\text { Excellent } \\
\left(r^{2} \geq 0.9995\right)\end{array}$ \\
\hline Sample Size & 794 & 9784 & 9914 & 558 \\
$T_{\text {ion }}(\mathrm{eV})$ & $0.09 \pm 0.01$ & $0.19 \pm 0.09$ & $0.19 \pm 0.09$ & $0.08 \pm 0.01$ \\
$A_{l}(\mathrm{MHz})$ & $368 \pm 363$ & $692 \pm 1103$ & $691 \pm 1,100$ & $398 \pm 358$ \\
$A_{u}(\mathrm{MHz})$ & $472 \pm 431$ & $670 \pm 1184$ & $670 \pm 1,180$ & $517 \pm 415$ \\
$B_{l}(\mathrm{MHz})$ & $772 \pm 5,036$ & $-334 \pm 10,565$ & $-331 \pm 10,537$ & $1,163 \pm 4,371$ \\
$B_{u}(\mathrm{MHz})$ & $459 \pm 4,230$ & $156 \pm 10,041$ & $159 \pm 10,019$ & $781 \pm 3,719$ \\
\hline
\end{tabular}

TABLE 5.1: Results of Monte Carlo analysis (described in text). Ion temperature, magnetic dipole coupling coefficients for upper and lower states, and electric quadrupole coupling coefficients are presented for excellent (RMSE $\leq 0.01$ or $\left.r^{2} \geq 0.9995\right)$, good (RMSE $\leq 0.05$ ), mediocre (RMSE $\leq 0.08)$ fits. The coupling coefficients in all cases are weakly constrained by this analysis.

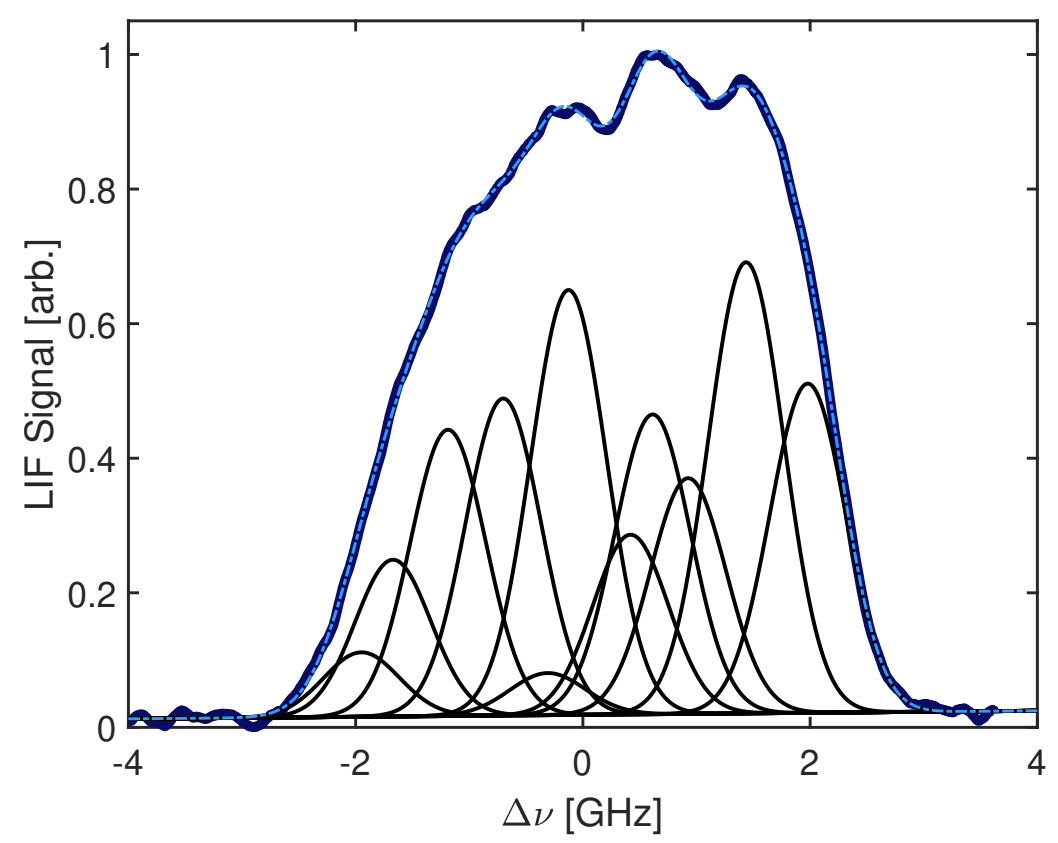

Figure 5.5: Typical I II measurement with best fit (lowest RMSE value) including (light blue dashed line) the 11 most dominant hyperfine peaks (black lines) reconstructed from this particular fit convergence. This is only one possible hyperfine structure configuration. This particular fit yielded a species temperature of $0.07 \mathrm{eV}$.

Additional attempts were made to isolate one or many of the hyperfine transitions spectroscopically. First, a form of saturation dip spectroscopy was used to try to measure expected decreases in the measured signal at the hyperfine rest frequencies. This Doppler-free method was used to eliminate the Doppler broadening from the measured lineshape to isolate individual peaks. Initially a counter-propagating injection was used in which the injection beam was reflected off of a mirror and passed back through the cell along the same path in an attempt to saturate the "zero-flow" population-creating a dip in the measured fluorescence at that location; this is sometimes also referred to as Lamb-dip spectroscopy. The experimental configuration for this 
method and a Lamb-dip graphic are shown in Fig. 5.6. However, the complex structure of this

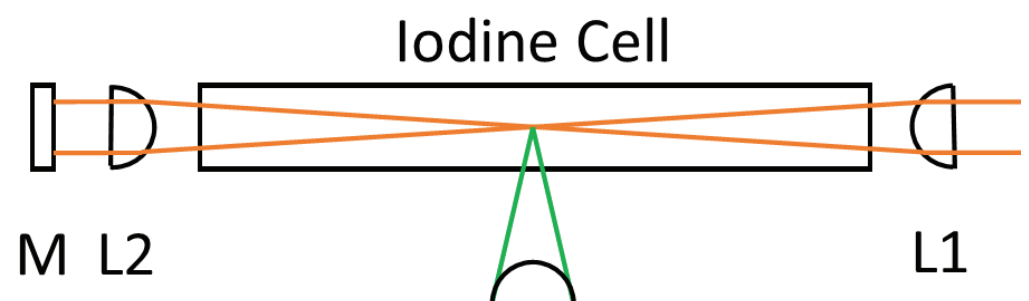

Collection

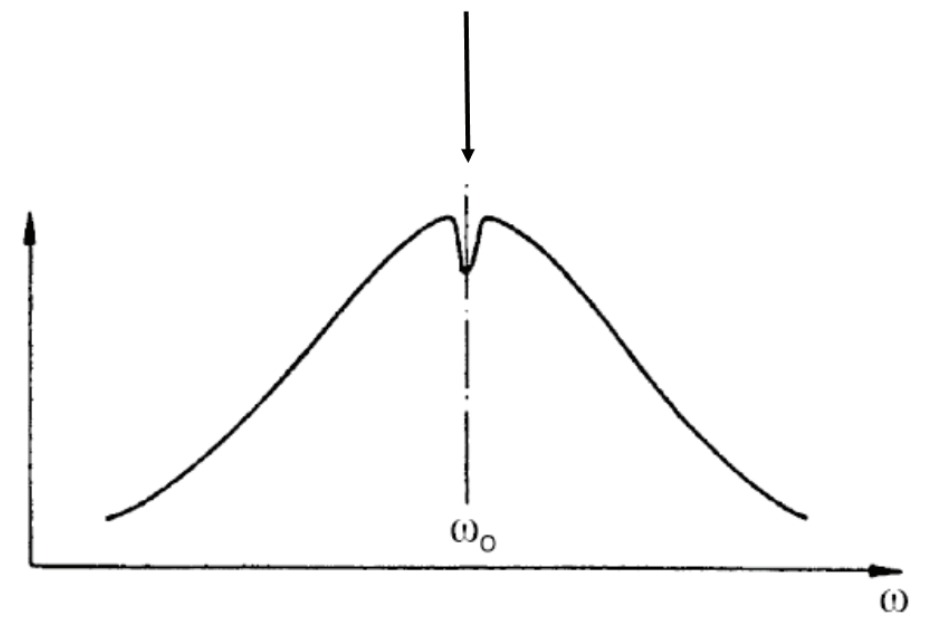

FIGURE 5.6: Experimental configuration for the saturation spectroscopy attempt. Injected laser is focused through a first lens (L1) with the focal point occurring near the center of the iodine cell. The light then diverges and is passed through a second identical lens and is collimated. The collimated light is reflected off a plane mirror and is passed through L2 again with the focal point overlapping with the initial incoming beam's focal spot. Fluorescence is collected perpendicularly in the region of the two overlapping focal spots. The particles that are at rest experience twice the amount of pumping which saturates the population making a deficit in the measured signal. A simple example of a Lamb-dip measured lineshape (adapted from Ref. [131] $)^{2}$ is shown below the schematic.

transition did not yield any noticeable Lamb-dip features. Figure 5.7 shows a IVDF collected with a standard single injection configuration (dark blue) compared to an IVDF measured using the focused saturated spectroscopic configuration. There are no discernible differences between the two measurements.

Figure 5.8 shows a theoretical spectral profile for different iodine ion temperatures. It can be readily seen that at the most probable temperature, the Doppler-broadened lineshape reveals no individual peaks. However, if the species temperature were to be reduced to room temperature $(0.026 \mathrm{eV})$ individual peaks are resolvable. The black lines in Fig. 5.8 are one possible hyperfine configuration as suggested from the Monte-Carlo fitting method. The increase in hyperfine

\footnotetext{
${ }^{2}$ Republished with permission of Springer, from work "Laser Spectroscopy 2," W. Demotroeder, 5th Ed. 2014. Permission conveyed through Copyright Clearance Center, Inc.
} 


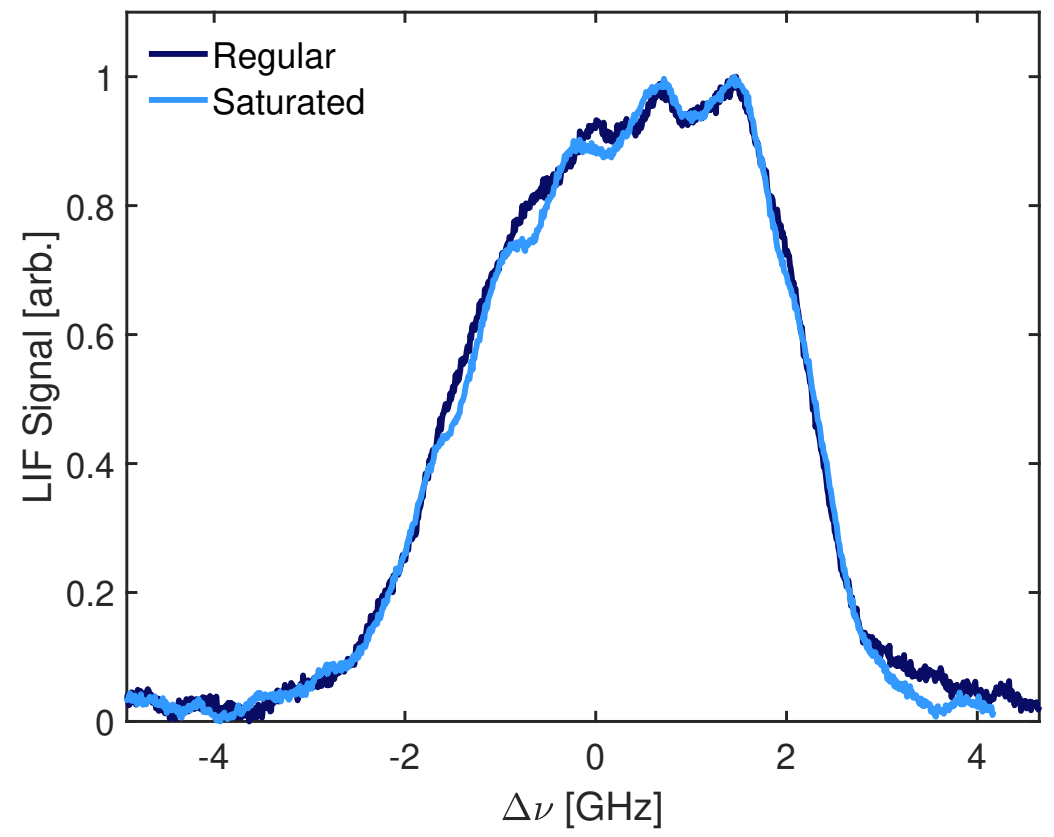

Figure 5.7: Iodine ion VDF measured with standard single injection configuration (dark blue) and dual-injection saturation (Lamb-dip) configuration (light blue) described in Fig. 5.6. There are no Lamb-dip features observed.

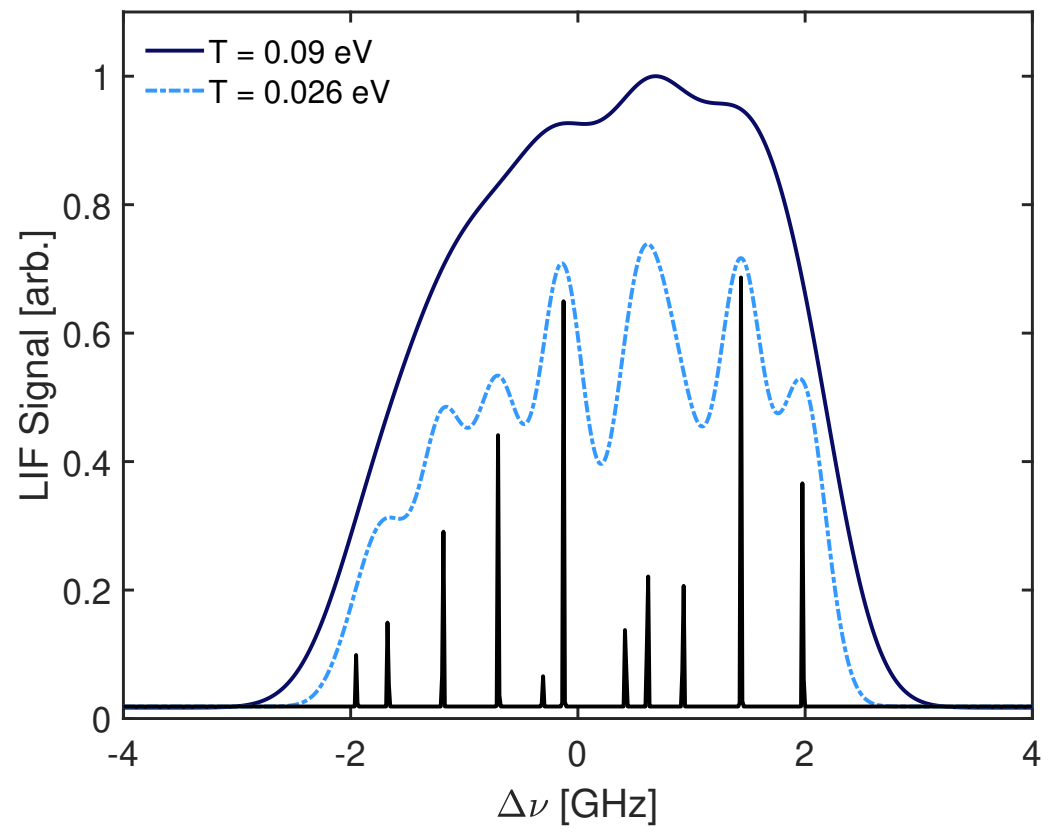

Figure 5.8: Two simulated profiles for an ion temperature of $0.09 \mathrm{eV}$ (most probable species temperature shown in dark blue) and simulated spectral lineshape for $0.026 \mathrm{eV}$ (light blue line). Black lines indicate a possible location for 11 of the 15 transitions as well as relative amplitudes of the hyperfine transitions. As the species temperature decreases, the resolvable hyperfine peaks are more apparent. Reproduced from Ref. [20]; reprinted by permission of the American Institute of Aeronautics and Astronautics, Inc.

resolution as species temperature decreases (or if Doppler broadening is completely removed) motivates attempting a method proposed in Ref. [132]. This technique relies on measuring two 
absorption profiles, one with a weak probe beam that measures the Doppler-broadened lineshape and one that measures a saturated lineshape. The experimental configuration for this technique and a cartoon depicting measured lineshapes are shown in Fig. 5.9. Two weak probe beams are

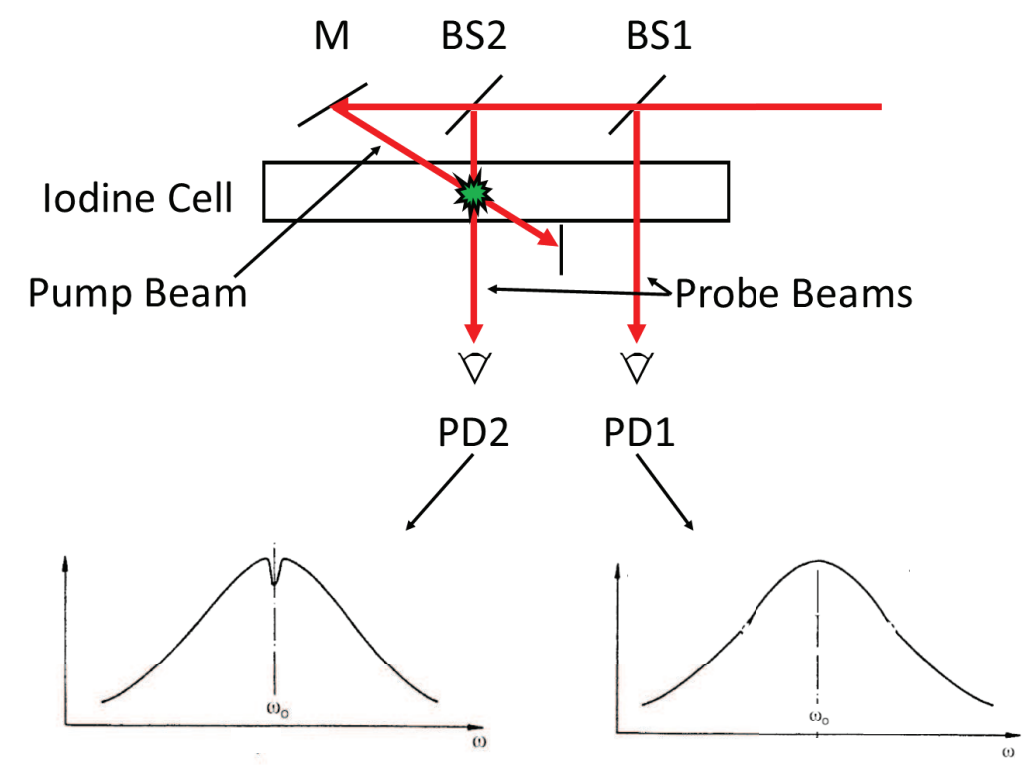

FiguRe 5.9: Experimental configuration for the Doppler-free saturation spectroscopic technique proposed in Ref. [132]. The injected laser light passes through a $\sim 90 / 10$ beamsplitting (BS1) where the 10\% of initial light that is split from the beam passes through the iodine cell and is incident on the first photodetector (PD1). As the laser wavelength is scanned, PD1 measures the absorption lineshape. The remainder of the pump beam passes through a second, identical beam splitter (BS2) creating a second probe beam that passes through the iodine cell and is incident on the second photodetector (PD2). The remaining pump beam reflects off a mirror (M) and passes through the iodine cell at some angle that intersects the second probe beam before terminating into a beam dump. The resulting absorption lineshape measured by PD2, in theory, displays a Lamb-dip at the rest frequency of the absorbers $\omega_{0}$. The subtraction of these two measurements yield the Doppler-free lineshape. ${ }^{3}$

split off from the pump beam. The first beam passes through the iodine cell and is incident on one of two photodetectors in a balanced photodetector. The second probe also passes through the iodine cell and is incident on the second photodetector, but is intersected by the remaining pump beam inside the cell. The resulting measured lineshape theoretically shows a saturation (Lamb) dip at the rest frequency. The balanced photodetector subtracts the two measured lineshapes and the resulting measurement is the Doppler-free lineshape. Unfortunately, this method was also unsuccessful as the balanced photodetector used was unable to measure an absorption profile. In LIF measurements, the Lock-in provides the means to differentiate small changes in background fluorescence. However, this measurement is fundamentally different since

\footnotetext{
${ }^{3}$ Republished with permission of Springer, from work "Laser Spectroscopy 2," W. Demotroeder, 5th Ed. 2014. Permission conveyed through Copyright Clearance Center, Inc.
} 
the measured absorption profile relies on a discernible drop in the probe beam power due to absorption. Boivin et al. provide an expression for the average optical depth of a plasma:

$$
\tau_{\circ}=\frac{n g_{1} A_{21} \lambda_{\circ}^{3}}{8 g_{2} \pi^{3 / 2} v_{\mathrm{thm}}} d
$$

where $n$ is the population density of the metastable state, $g_{1}$ and $g_{2}$ are the statistical weights of the lower and upper states of the pump transition, respectively, $A_{21}$ is the spontaneous emission coefficient of the upper state to the lower state, $\lambda_{\circ}$ is the rest frame wavelength of the transition, $d$ is the path length of the light through the plasma, and $v_{\text {thm }}=\sqrt{2 k_{b} T_{i} / m_{i}}$ is the thermal velocity of the ion ( $k_{b}$ is Boltzmann's constant, $T_{i}$ is the ion temperature, and $m_{i}$ is the iodine mass) [133]. Many of these quantities are not known for the iodine cell, so values from similar experiments are used to estimate the optical depth. The gas density is taken to be similar to a cell investigated by Wu et al. [134] calculated using Killian's equation [135]. This would suggest the cell is weakly ionized, $\sim 1 \%$ ionization. The spontaneous emission coefficient is assumed to take a value of $10^{7}-10^{8} \mathrm{~s}^{-1}$. The thermal velocity is calculated using the most probable ion temperature from the Monte-Carlo fitting analysis. The path length is the diameter of the cell, $12 \mathrm{~mm}$. With these assumptions, it is estimated that a reduction in transmitted laser power of $0.2-2 \%$ is probable and is the likely reason for lack of absorption measurements using this technique. Therefore, I II temperature is not definitively measured in this work, only estimated. However, bulk ion flow can still be determined from the relative shift of the measured lineshape.

The centroid for the hyperfine broadened lineshape was determined to benchmark a "zero-flow" for this transition. Since measurements are within a sealed static cell, it was assumed initially that the measurements (regardless of laser injection direction) provide this static benchmark value. The hypothesis was confirmed by injecting the laser beam anti-parallel to its initial injection path and observing no spectral shift. Figure 5.10 shows two measured I II lineshapes for two colinear, anti-parallel laser injection paths. To this end, a spectral center for the I II transition probed in this work is provided with higher resolution than previously quoted and is in agreement with the value measured in Ref. [57]. The rest transition's wavelength was determined to be $696.0694 \pm 0.0002 \mathrm{~nm}$ [20], improving on the previous measurement resolution of $696.070 \pm 0.001 \mathrm{~nm}$ [57]. These data provide a reliable, high-precision spectral center for accurate I II flow measurements in thrusters. 


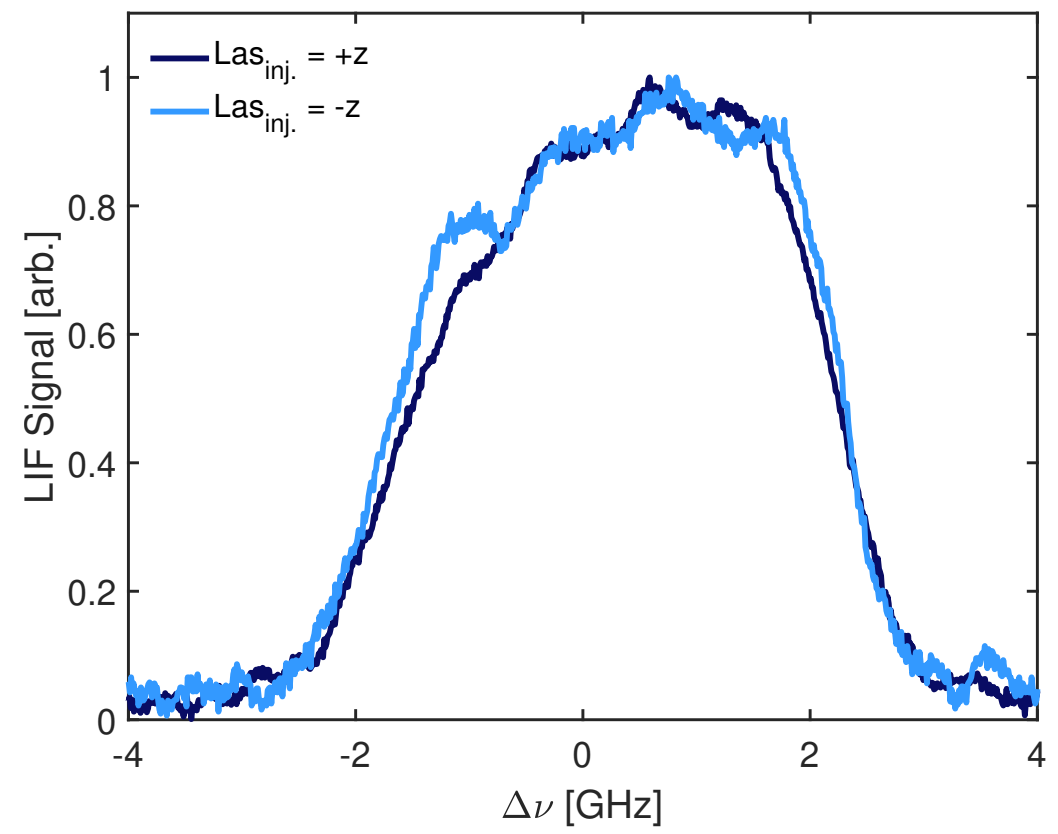

Figure 5.10: LIF measurements for laser injection propagating along the $+z$ axis (dark blue) and $-z$ axis (light blue). No discernible spectral shift is observed for the two injection paths, consistent with zero bulk flow within the iodine cell.

\subsection{Zeeman-split I II LIF}

Hall thrusters will inherently alter the measured lineshape with the introduction of the external magnetic field that is fundamental to HT operation. In typical Zeeman-split spectra, hyperfine splitting is a negligible effect and complications from large Zeeman splitting, i.e., $\sigma$ peaks, is often eliminated with simple polarizing optics. However, in this work the complex hyperfine structure introduces significant effects even for the less extreme $\pi$-split transitions. Typically, $\pi$ splitting is symmetric about the rest transition frequency and, as a result, the bulk flow determined is largely unaffected. Because the $\sigma$-peak splitting is linearly proportional to the external magnetic field strength, measurements of the Zeeman splitting provides a means to perform a non-perturbative magnetic field measurements inside a thruster [53, 94].

The hyperfine structure of the I II transition adds an incredible amount of complexity to the measured Zeeman-split lineshape. The combined Zeeman-hyperfine structure was investigated with a variable external magnetic field applied perpendicularly to the cell's mechanical axis. Figure 5.11 shows an example Zeeman-split measured lineshape (dark blue) along with a measured lineshape in the absence of an external magnetic field. The magnetic field strength for this 
measurement is $\sim 3 \mathrm{kG}$. It is apparent that all the hyperfine lines do not have the same magnitude of response to the external magnetic field. In Fig. 5.11 primary $\pi$ (when laser polarization is parallel to the magnetic field direction $\hat{b}$ ) and $\sigma$ (when laser polarization is perpendicular to $\hat{b}$ ) clusters are observed with additional smaller clusters split even further than the primary peaks. These additional clusters are present on both sides of any given primary cluster (i.e., any $\pi$ or $\sigma$ peak). This is demonstrated in Fig. 5.12. All Zeeman spectra were collected at a gas pressure $\mathrm{P}_{\text {gas }}=11$ mTorr.

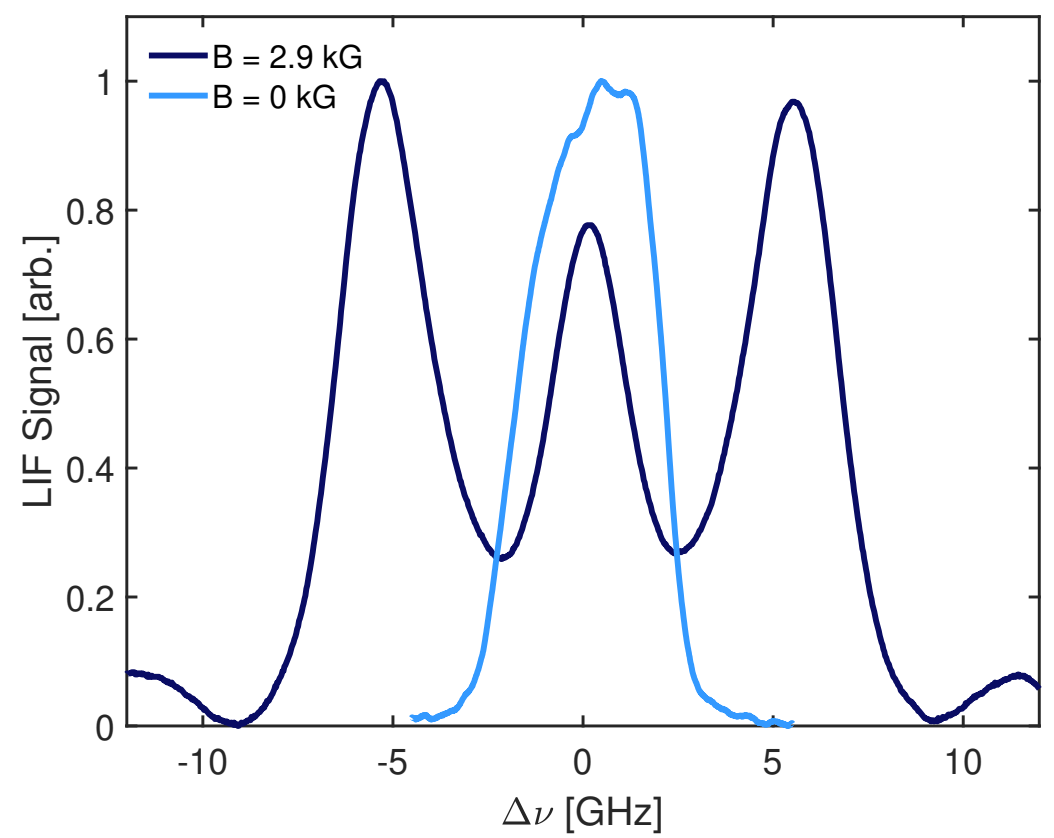

Figure 5.11: Example Zeeman-split spectrum (dark blue) compared to a typical lineshape in the absence of an external magnetic field (light blue). The Zeeman-split spectrum is complex and significantly split. As with the regular lineshape, the structure is not able to be characterized due to unknown hyperfine coupling coefficients. These data were taken at a pressure of $\mathrm{P}_{\text {gas }}=11 \mathrm{mTorr}$ and a microwave power $\mathrm{P}_{\mathrm{MW}}=30 \mathrm{~W}$.

While the exact structure of the Zeeman-split I II transition is not determined here, the separation of the primary $\sigma$-peaks is an excellent measure of the external magnetic field. The applied magnetic field was varied from $\sim 0.270 \mathrm{kG}$ to $\sim 3 \mathrm{kG}$ and the separation of $\sigma$-peaks was used to determine a Zeeman-splitting factor $\epsilon_{\mathrm{z}} \cdot \sigma$-peak overlap occurs at magnetic field strengths less than $1 \mathrm{kG}$. If in addition to a linear polarizer a $\lambda / 4$-wave plate is inserted into the collection optics a single $\sigma$-peak is isolated. A rotation of the $\lambda / 4$-wave plate by $90^{\circ}$ selects the opposite $\sigma$-peak. This method theoretically extends minimum determinable field to $\sim 0.1$ kG. Figure 5.13 shows the primary $\sigma$-peak separation for applied magnetic field strengths of $\sim 1 \mathrm{kG}$ to $\sim 3 \mathrm{kG}$. Black dashed lines indicate the primary $\sigma$-peak cluster locations. From 


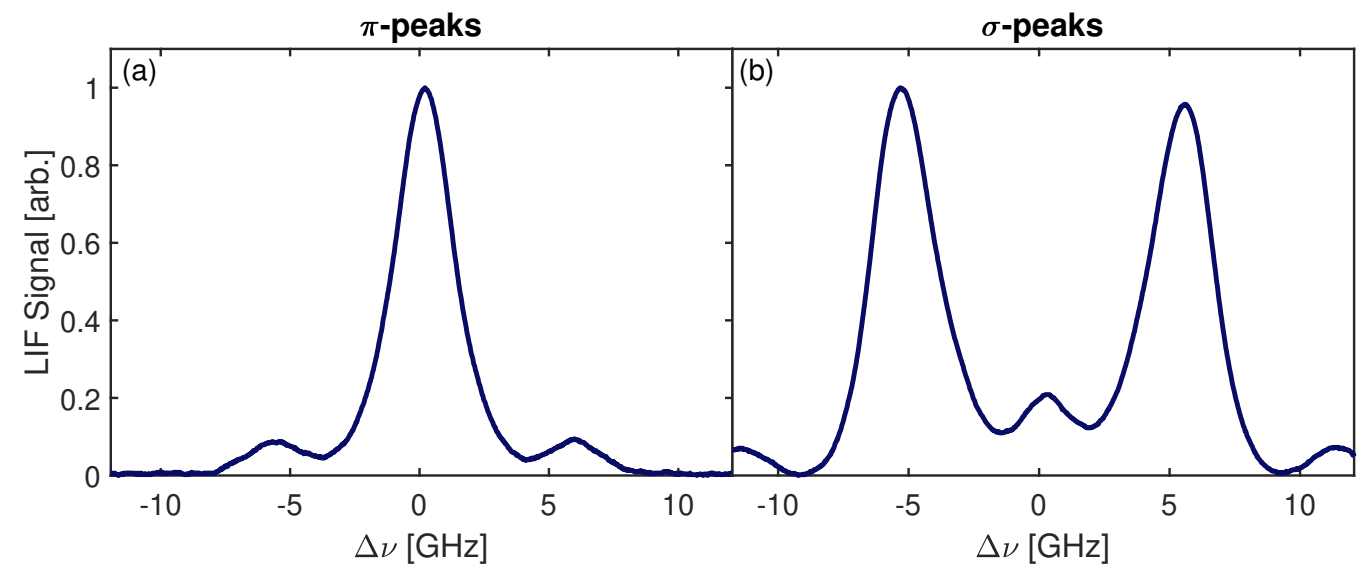

FiguRe 5.12: $\pi$ (a) (polarization $\| \hat{b}$ ) and $\sigma($ b) (polarization $\perp \hat{b}$ ) clusters for the I II Zeeman-split spectrum. Secondary clusters are observed in the wings of both $\pi$ and $\sigma$ spectra, suggesting that not all the hyperfine transitions have the same susceptibility to an external magnetic field.

these data, the Zeeman-splitting constant is determined to be $1.9 \pm 0.1 \mathrm{GHz} / \mathrm{kG}$. Figure 5.14 shows the Zeeman-splitting values as a function of magnetic field strength.

Typical magnetic field strengths in HTs are measured to be $100-200 \mathrm{G}$ [136]. Commonly, these fields are measured using a $\dot{B}$ (B-dot) probe. However, this method perturbs the plasma. The magnetic field diagnostic presented here is non-perturbative and spatially localized. While the minimum detectable magnetic field strength measured in this work is significantly higher than typical fields measure in HT, lower magnetic field strengths are able to be determined from a Zeeman-split spectrum using a polarizer- $\lambda / 4$-wave plate combination. This optical configuration isolates a single $\sigma$-peak. Rotating the $\lambda / 4$-wave plate selects the opposite $\sigma$-peak and thus the overlap of $\sigma$-peak lineshapes does not occur for a single measurement. The theoretical minimum detectable magnetic field using this technique is $\sim 100$ G. Confocal measurements of the I II distribution function (and Zeeman-split spectra) provide means to measure plasma quantities within the channel of a HT, where only a single line-of-site is possible.

\subsection{Summary}

I II velocity distributions were measured for the first time using LIF. A custom vacuum sealed reference cell filled with iodine was constructed with a cooling "finger" to adjust the internal iodine partial pressure. Iodine LIF signal was inversely proportional to iodine partial pressure. Measurements reveal that the hyperfine structure of the interrogated transition is significant and leads to an exceptionally broadened lineshape. Individual hyperfine transitions were not able to 


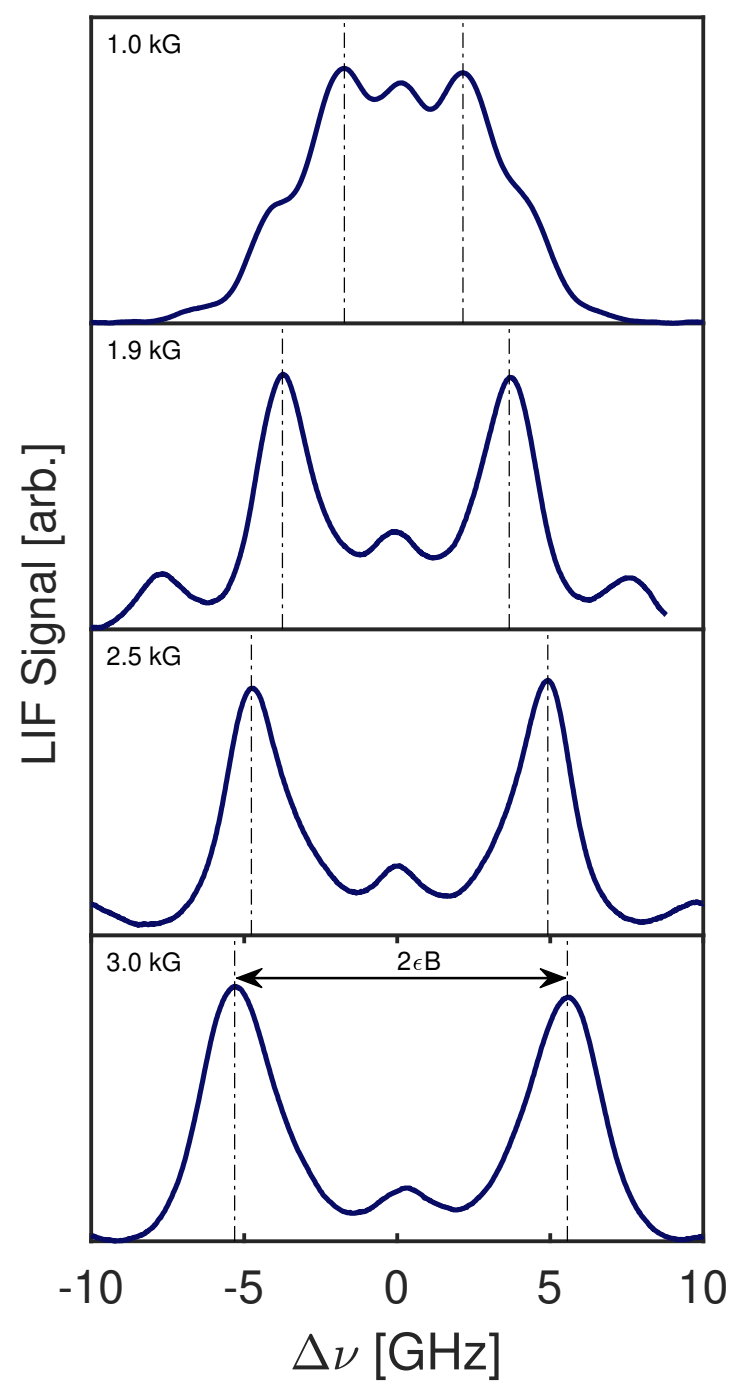

Figure 5.13: Primary $\sigma$-peak cluster separation for magnetic fields ranging from $1 \mathrm{kG}$ to 3 $\mathrm{kG}$. Black dashed lines indicate $\sigma$-peak locations. $\sigma$-peaks overlap for magnetic fields less than 1 kG. Reproduced from Ref. [94], with the permission of AIP Publishing.

be resolved and hyperfine coefficients are not known. Fifteen separate hyperfine transitions make up the measured profile, with eleven of the fifteen predicted to make significant contributions to the lineshape. The ion temperature was estimated by fitting the measured lineshape to a function comprised of eleven Gaussian features that was constrained to satisfy the predictions for hyperfine splitting. The resultant most-probable ion temperature was $\sim 0.1 \mathrm{eV}$. Hyperfine parameters were weakly constrained by this method.

Zeeman splitting of the probed transition was also investigated. The magnitude of the splitting of $\sigma$ clusters is directly proportional to the external magnetic field. In this way, it is possible to use this diagnostic to determine the bulk flow of iodine and the magnetic field in an iodine plasma thruster. Since the hyperfine splitting also splits in the presence of the magnetic field, 


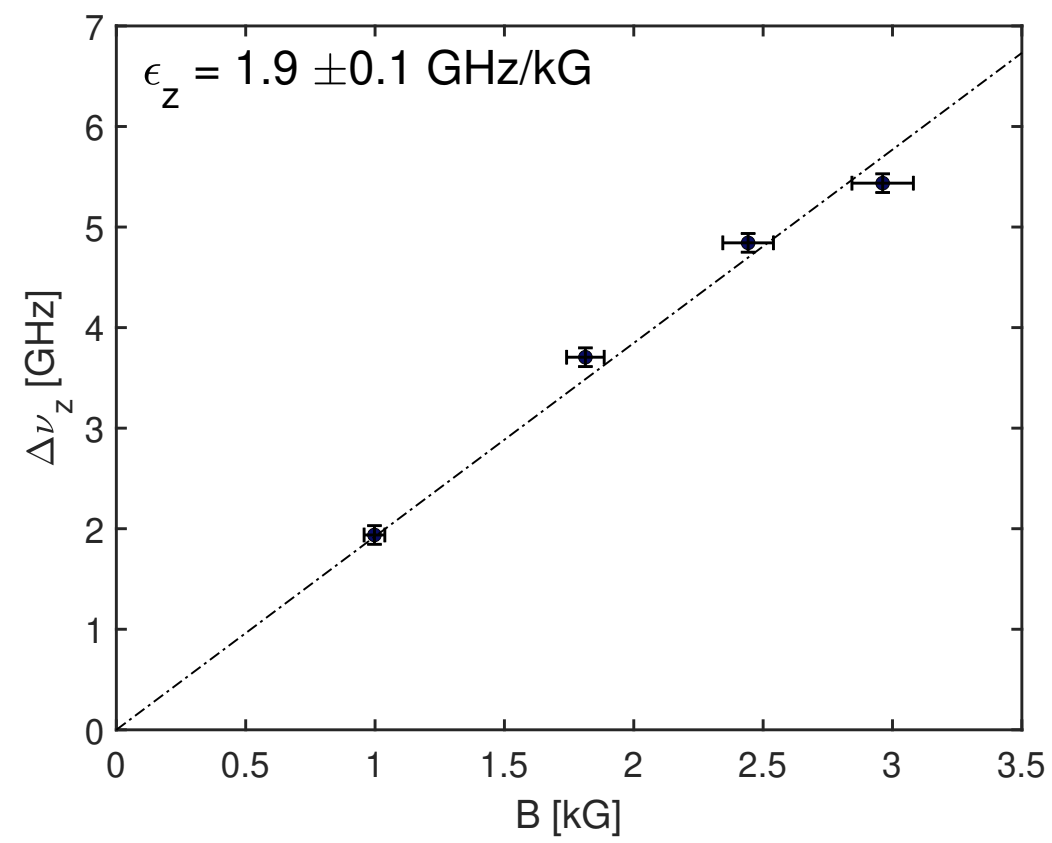

Figure 5.14: Zeeman splitting as a function of applied magnetic field. The Zeeman-splitting constant is determined to be $1.9 \pm 0.1 \mathrm{GHz} / \mathrm{kG}$. Reproduced from Ref. [94], with the permission of AIP Publishing.

the Zeeman-split spectrum is also significantly broad and to diagnose magnetic fields less than $\sim 1 \mathrm{kG}$ requires additional optics to isolate individual $\sigma$-peaks. 


\section{Chapter 6}

\section{Results: Deuterium TALIF}

Deuterium VDFs were measured with TALIF with a laser-to-chamber optical path of $\sim 20 \mathrm{~m}$. Proto-MPEX was operated significantly outside its typical parameter space to obtain acceptable deuterium SNR. These measurements were plagued by several obstacles that impeded TALIF measurements at standard Proto-MPEX operational parameters. TALIF measurements were performed for a range of radial locations in Proto-MPEX and for a range of rf powers.

\subsection{Xenon Calibration in Proto-MPEX}

The original experimental plan for deuterium TALIF on Proto-MPEX included calibrating the measurements for absolute density using xenon as the calibration gas. Xenon TALIF measurements were performed, but the results had poor SNR. There are many factors that contribute to the poor SNR of the xenon TALIF measurements. The transition selected for xenon has a two-photon cross section that is much smaller than the cross sections for krypton or deuterium. Additionally, the laser energy produced at the wavelengths required to excite the xenon TALIF transition is $\leq 1 \mathrm{~mJ} / \mathrm{pls}$ compared to $\geq 2.5 \mathrm{~mJ} /$ pls for krypton and deuterium pump wavelengths. Note that TALIF signal is proportional to laser pulse energy squared. Additionally, when the laser gratings were tuned to pump xenon $(\lambda \sim 630 \mathrm{~nm})$, significant background light was detected by the double-filtered PMT. This effect made fine alignment for xenon nearly impossible since a detectable pulse is produced from the PMT at the same time the laser pulse is present, and is seemingly as strong as any fluorescence signal. A typical xenon VDF measured in Proto-MPEX at a pressure of 100 mTorr is shown in Fig. 6.1. Error bars represent the 
standard deviation of the TALIF signal at a given frequency. The large uncertainties in the xenon TALIF measurements introduce significant errors into any attempts to use xenon as the calibration gas.

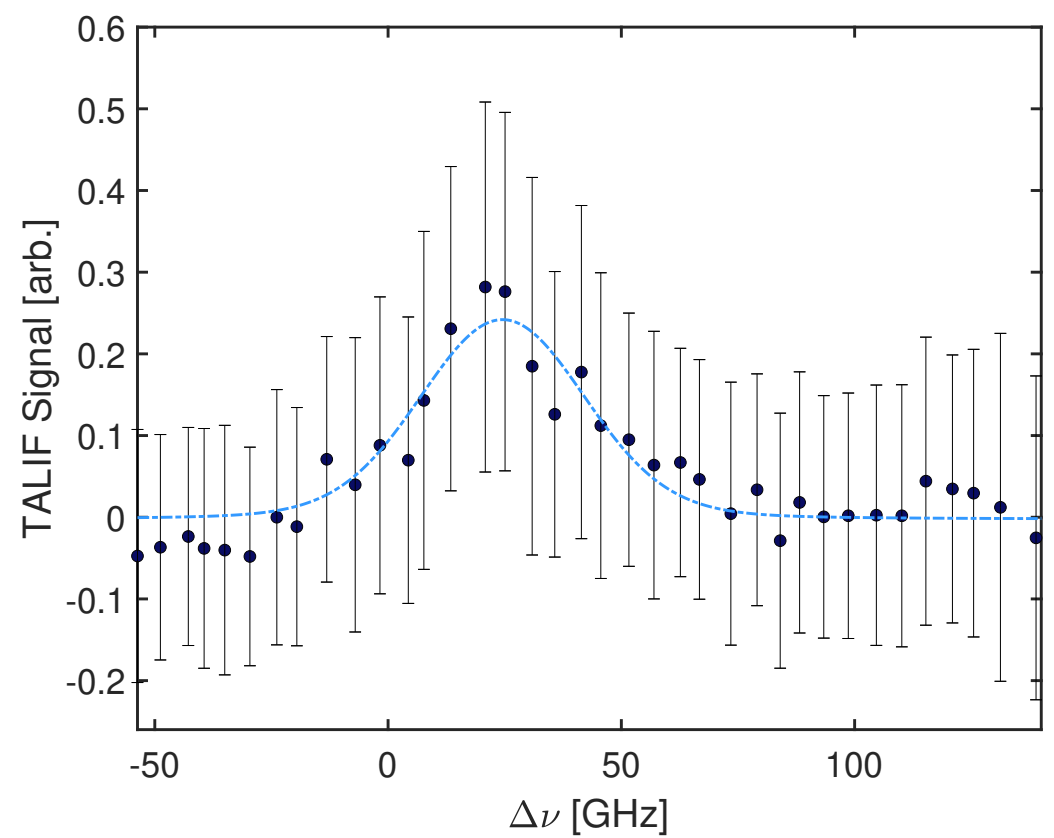

Figure 6.1: Typical Xe NVDF measured in Proto-MPEX at a static fill pressure of 100 mTorr. SNR is poor due to the significant variation of signal caused by broadband emission around the fluorescence wavelength of Xe.

Several attempts were made to mitigate and eliminate the mysterious red light reaching the TALIF detector during xenon calibration. Initially, it was thought that residual dye-converted laser light was, in some manner, transmitted over the $20 \mathrm{~m}$ beamline, collected by the confocal apparatus, and returned to the PMT. For xenon pumping, the wavelength is $\sim 627 \mathrm{~nm}$ and the bandpass filter in front of the PMT rejects all light around $656 \mathrm{~nm}$ with a $1 \mathrm{~nm}$ FWHM pass band to an optical depth (OD) 5 level. It was hypothesized that some $627 \mathrm{~nm}$ light was making it through the $1 \mathrm{~nm}$ filter and creating a false TALIF signal. To address this possible source of contamination, a second $1 \mathrm{~nm}$ narrowband filter and a third highpass filter with a cutoff wavelength at $630 \mathrm{~nm}$ were placed before the PMT. Even with these additional filters, the contamination still persisted. The reduction in SNR because of the reduced transmission was also unacceptable. Therefore just the two $1 \mathrm{~nm}$ wide bandpass filters were employed for the measurements presented here (except for krypton measurements, where only one $10 \mathrm{~nm}$ wide bandpass filter was used). 
Upon further investigation, the background pulse was present for all dye laser wavelengths and only disappeared for deuterium and krypton measurements because the PMT voltage was lower due to the brighter TALIF emission for these species. This suggests that the collected light at the injection optics detected by the PMT is not intense residual dye light, but rather broadband light. The light is most likely caused by laser-stimulated emission from air and surfaces in the injection optics. Non-reflective materials were investigated at the injection site and measurements yielded no significant reduction of the background light. Some materials did mitigate this issue, but only marginally. Because of the poor SNR, the xenon calibration measurements are only provided here for completeness and the absolute density values should be derived from krypton calibration. Figure 6.2 shows a typical krypton NVDF measured in Proto-MPEX at $\sim 2.5$ mTorr. It is emphasized here that the krypton measurement shown here is at nearly $50 \times$ less fill pressure than the xenon measurement shown above and nearly two orders of magnitude less gain on the PMT, e.g., krypton fluorescence is much stronger than xenon fluorescence and provides a more reliable calibration benchmark.

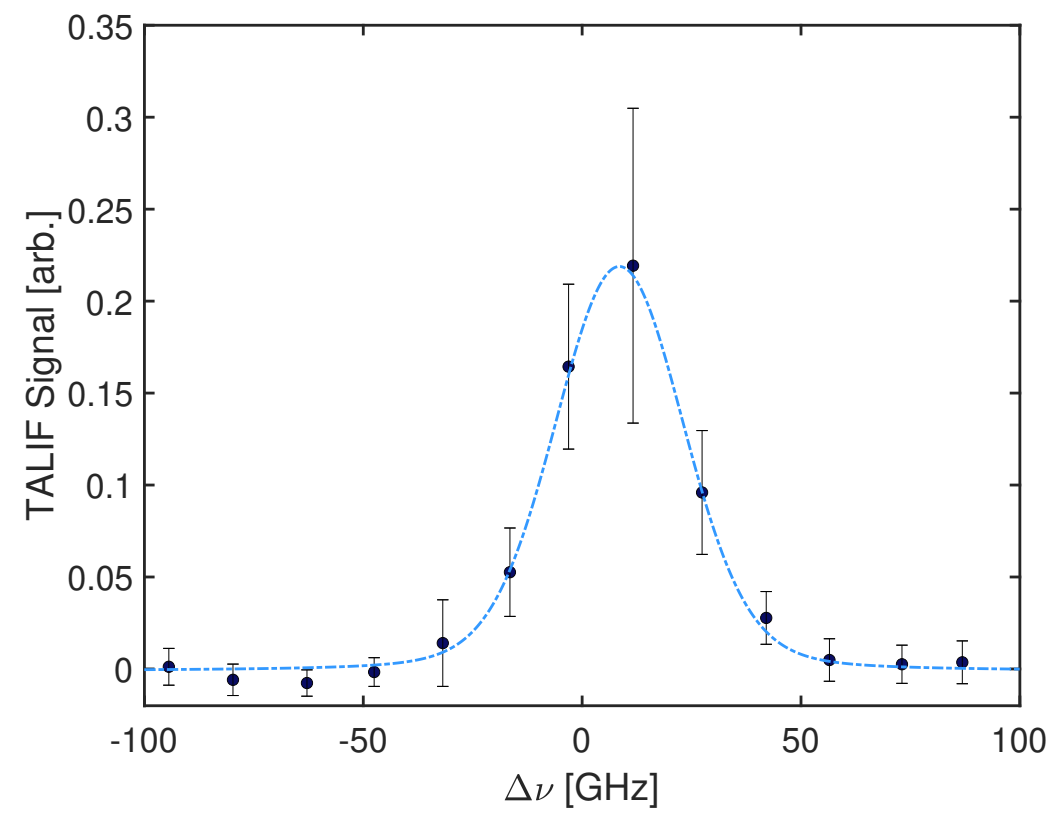

Figure 6.2: Typical Kr NVDF measured in Proto-MPEX at a static fill pressure of 2.5 mTorr. SNR is significantly better than Xe measurements measured at Proto-MPEX. 


\subsection{Proto-MPEX Operation for Preliminary TALIF Measure- ments}

The parameter space of Proto-MPEX used in this work is significantly different from standard Proto-MPEX operation. Specifically, only low rf powers $(\leq 60 \mathrm{~kW})$ and gas pressures greater than 40 mTorr produced favorable conditions for deuterium TALIF measurements. Two separate gas puffers supply a substantial amount of gas over a $1 \mathrm{~s}$ plasma pulse in Proto-MPEX. A typical evolution of the gas pressure in Proto-MPEX for the measurements made in this work is shown in Fig. 6.3. The pressure increases to over 100 mTorr over the entire pulse length of the plasma. Deuterium TALIF signals are on the order of the noise until $t=500 \mathrm{~ms}$ for all radial locations for larger rf powers. For lower rf power, discernible deuterium TALIF signal is observed at earlier times. Proto-MPEX ionizes deuterium efficiently at normal operation. Even

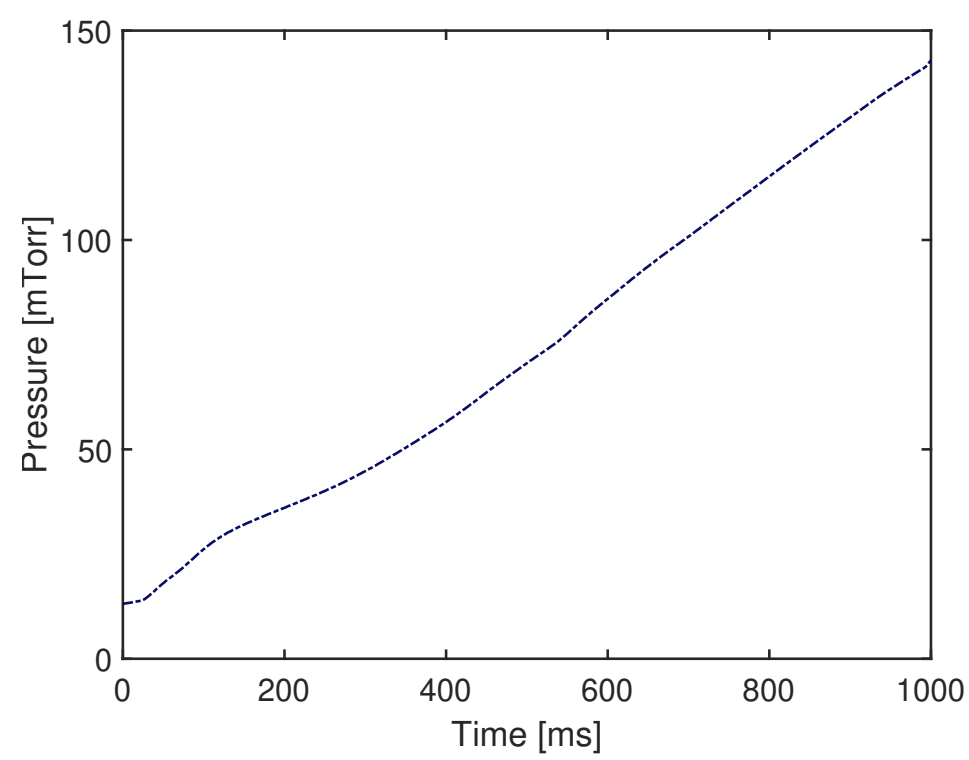

Figure 6.3: Evolution of the pressure profile in Proto-MPEX for the TALIF measurements presented here. Pressure increases to over 100 mTorr over the entirety of the plasma pulse. Discernible TALIF signal is not typically detected for times less than $\sim 500 \mathrm{~ms}$.

at the lowest rf powers investigated here, background $\mathrm{D}_{\alpha}$ was significant and dictated low PMT voltages to avoid detector saturation. Efforts to gate the PMT around the laser pulse improved permissible PMT voltage marginally. Therefore, the TALIF measurements reported here were performed in atypical Proto-MPEX conditions.

This long Proto-MPEX plasma pulse length allowed for measurements for 20 laser pulses. Full NVDFs were constructed from ten distinct wavelengths. Figure 6.4 shows a typical deuterium 
NVDF obtained from average over all 20 laser pulses at $r=2 \mathrm{~cm}$ at $30 \mathrm{~kW}$. The error bars are the standard deviation of the signal at each given frequency for the 20 pulses. For this particular shot, the neutral temperature was measured to be $0.2 \mathrm{eV}$, well below theorized dissociated neutral hydrogen temperatures of $\sim 1 \mathrm{eV}[74]$. The neutral density for this measurement, while time averaged over the entire plasma pulse, is calculated to be $6.73 \times 10^{20} \mathrm{~m}^{-3}$ when calibrated with xenon and $7.24 \times 10^{20} \mathrm{~m}^{-3}$ for krypton. The two absolute densities are in excellent agreement with each other. The bulk flow time averaged over the plasma pulse is approximately $2.7 \mathrm{~km} / \mathrm{s}$ radially inward. Ion flows have been predicted and observed to flow axially away from the helicon region (both upstream and downstream) in Proto-MPEX [66] and the neutral flow measurements presented here are consistent with this ion flow. As the ion population moves axially upstream or downstream, neutrals rush radially inward to sustain helicon operation.

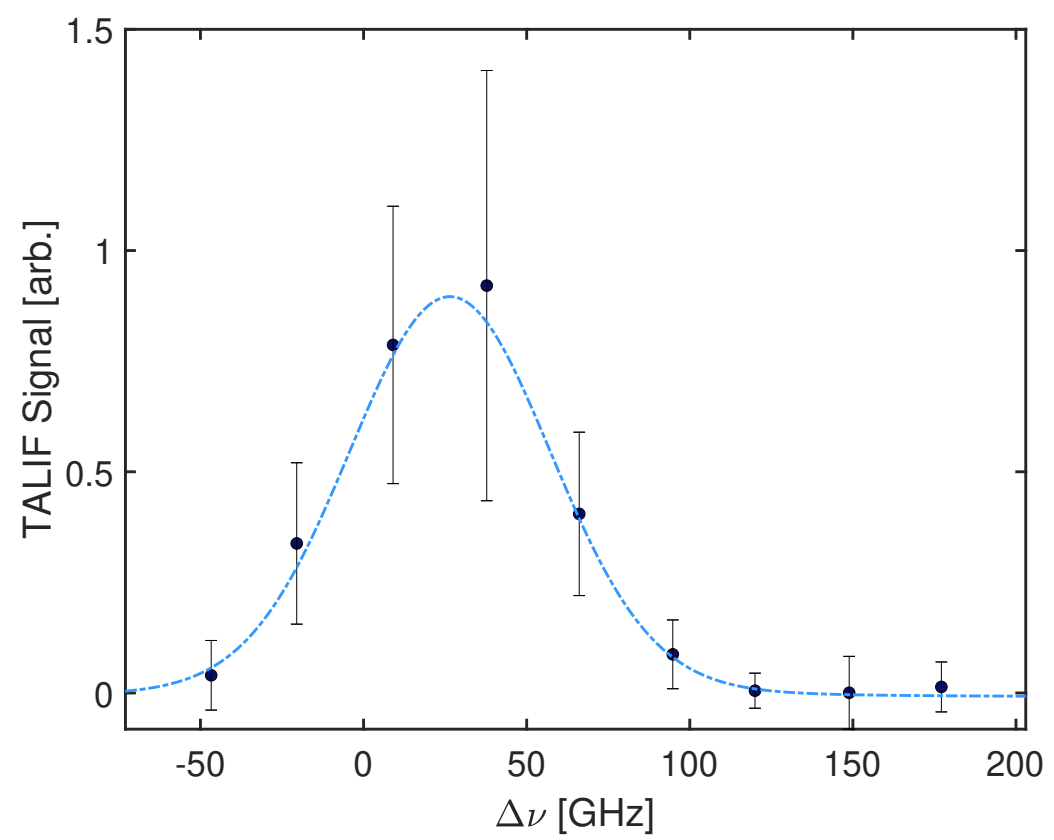

Figure 6.4: Typical deuterium NVDF measured on Proto-MPEX. Error bars are the standard deviation of the variation of signal intensity. Since signal increases over the entire plasma pulse, a temporal plot for every $250 \mathrm{~ms}$ is the preferred method of data representation. This measurement was taken at $2 \mathrm{~cm}$ off axis at an rf power of $30 \mathrm{~kW}$.

Since the TALIF signal intensity evolves over the plasma pulse, every 5 laser pulses are grouped and averaged together to produce a temporal evolution of neutral deuterium NVDF with a time resolution of $250 \mathrm{~ms}$. The temporal evolution of the deuterium NVDF shown in Fig. 6.4 is depicted in Fig. 6.5. The data shown in Fig. 6.5 were taken at $r=2$ off axis and at an $\mathrm{rf}$ power 


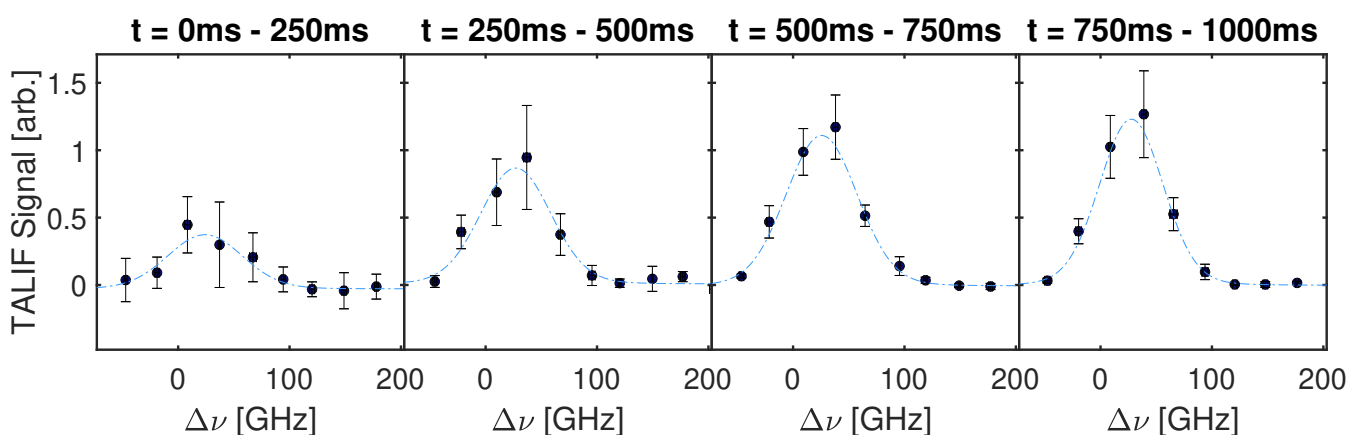

FiguRe 6.5: Example temporal evolution of a deuterium NVDF on Proto-MPEX. Each data point in each subplot is an average of 5 laser pulses revealing neutral deuterium dynamics on the time scale of $250 \mathrm{~ms}$. This figure shows discernible signal for all times. It is equally as common to only observe signal for later times, especially at higher rf powers. This measurement was taken at $2 \mathrm{~cm}$ off axis at an rf power of $30 \mathrm{~kW}$.

of $30 \mathrm{~kW}$. In Fig. 6.5, the neutral density increases for the first $750 \mathrm{~ms}$ and remains constant for the last $250 \mathrm{~ms}$. For this particular measurement, the neutral deuterium temperature decreases over the plasma pulse. Measured bulk flow is essentially constant for the entire plasma pulse. Comparison of this measurement to other radial locations is discussed later in this chapter.

\subsection{RF Power Variation Measurements}

Deuterium NVDFs were collected for four rf powers. At the time of these measurements, 30 $\mathrm{kW}$ was the lower bound for Proto-MPEX operation to satisfy power supply interlocks. At the opposite extreme, deuterium TALIF signal was unable to be detected at rf powers above 60 $\mathrm{kW}$. This is most likely due to the increased ionization with increasing rf power. Therefore, the rf operating space for deuterium TALIF measurements presented here is $30 \leq \mathrm{P}_{\mathrm{RF}} \leq 60 \mathrm{~kW}$ for any radius.

Figure 6.6 shows the temporal evolution of the neutral deuterium velocity distributions for four different rf powers. Data shown for $\mathrm{P}_{\mathrm{RF}}=30 \mathrm{~kW}$ were taken several days apart from the measurements corresponding to $\mathrm{P}_{\mathrm{RF}}=40-60 \mathrm{~kW}$. Differences in lineshape intensity occur from day-to-day operation and have several possible causes. It is for this reason that NVDFs are calibrated with shots from the same data run and same day. Low $\mathrm{P}_{\mathrm{RF}}$ produced sufficient signal for all times. For higher $\mathrm{P}_{\mathrm{RF}}$, significant signal was only observed at later times in the plasma pulse. The absolute neutral density and temperatures are listed for NVDFs with acceptable fits. When possible, absolute densities calibrated with both krypton and xenon are compared. 
In Fig. 6.6, $\mathrm{P}_{\mathrm{RF}}=60 \mathrm{~kW}$ is shown in row (a), $\mathrm{P}_{\mathrm{RF}}=50 \mathrm{~kW}$ is shown in row (b), $\mathrm{P}_{\mathrm{RF}}=40$ $\mathrm{kW}$ is shown in row (c), and $\mathrm{P}_{\mathrm{RF}}=30 \mathrm{~kW}$ is shown in row (d).

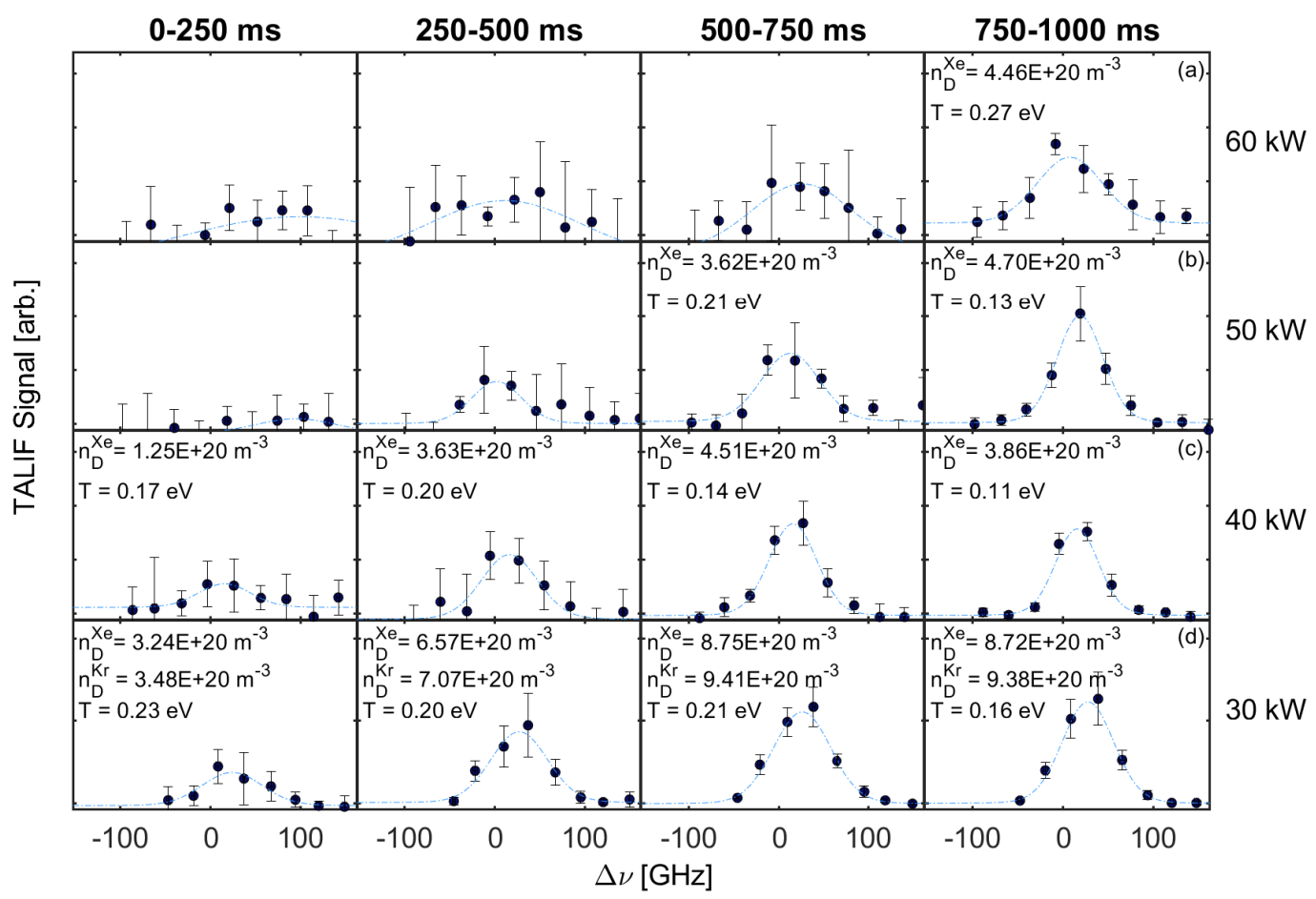

Figure 6.6: Deuterium NVDF evolution over the plasma pulse for (a) $\mathrm{P}_{\mathrm{RF}}=60 \mathrm{~kW}$, (b) $\mathrm{P}_{\mathrm{RF}}=50 \mathrm{~kW}$, (c) $\mathrm{P}_{\mathrm{RF}}=40 \mathrm{~kW}$, and (d) $\mathrm{P}_{\mathrm{RF}}=30 \mathrm{~kW}$. Absolute density and temperature are shown for every acceptable fit to the data. Lower powers produce sufficient signal for all times during the plasma pulse, whereas only later times exhibit discernible signal for higher powers. Comparison between xenon and krypton calibrated shots are shown where available.

These data are shown for a constant radial position of $r=2 \mathrm{~cm}$. Each NVDF is determined using the same method discussed for Fig. 6.5. Generally, significant TALIF signal is only observed for later times in the plasma pulse, i.e., $t \geq 500 \mathrm{~ms}$. However, for an $\mathrm{rf}$ power of 30 $\mathrm{kW}$, discernible TALIF signal is observed earlier in the plasma pulse. Absolute neutral density is determined using both xenon $\left(n_{\mathrm{D}}^{\mathrm{Xe}}\right)$ and krypton $\left(n_{\mathrm{D}}^{\mathrm{Kr}}\right)$ for an rf power of $30 \mathrm{~kW}$. Derived neutral densities from these two gases are in excellent agreement with each other. For all other rf powers, absolute densities are derived from xenon only. Neutral deuterium density increases throughout the plasma pulse, in agreement with the measured pressure trace, though the dependence is weak. As the rf power increases, the measured deuterium density decreases, as expected due to greater ionization for larger rf powers. Neutral deuterium temperature is nominally $0.2 \mathrm{eV}$ and decreases for later times in the plasma pulse. This is expected since for later times the plasma is oversaturated with gas and starts to enter an afterglow regime. During these later 
times, the neutrals are less affected by heating processes (charge exchange, for example) and start to relax to the temperature of the chamber walls. The neutral temperatures measured here are in good agreement with temperatures estimated for molecular neutrals in the edge of Proto-MPEX [137]. In Ref. [137], Proto-MPEX wall temperatures are fixed at $0.1 \mathrm{eV}$ and molecular deuterium temperatures in the edge of the plasma column for typical Proto-MPEX operation is slightly higher on average. Bulk neutral flow at $r=2 \mathrm{~cm}$ is radially inward and monotonically decreases as rf power increases, but is constant for the entirety of the plasma pulse for a given rf power. For $\mathrm{P}_{R F}=60 \mathrm{~kW}$, neutral temperature is measured to be $\sim 0.3 \mathrm{eV}$, considerably lower than Franck-Condon neutrals, but consistent with molecular temperatures estimated near the edge of the plasma column [137].

Shown in Fig. 6.7 are the measured absolute densities versus rf power for each time interval. Note the difference in measured neutral densities for $\mathrm{P}_{\mathrm{RF}}=50 \mathrm{~kW} t=500-750 \mathrm{~ms}$. The two data points shown for $\mathrm{P}_{\mathrm{RF}}=50 \mathrm{~kW}$ were taken nearly eight hours apart (one at the beginning of operation and the other at the end). While the density values lie within their error bars, they are noticeably different for the same conditions. The difference is likely due to their lack of reproducibility of the plasma source over several shots and the unreliability of the laser energy calibration factor. Over a long operation day, as shown in the lower portion of Fig. 6.7, the pressure evolution over the plasma pulse differs by $\sim 10$ mTorr at some times for the two $50 \mathrm{~kW}$ cases. Thus, the source is not always reproducible. Prolonged operation of the laser changes the efficiency of the conversion crystals, which can be corrected by small changes to the motor position of the crystals. However, this requires new tuning tables to be created multiple times a day. The efficiency loss is not observed at the dye stage (where the wavemeter samples the laser energy). Since the method of determining laser energy per pulse at Proto-MPEX relies on a valid conversion between the dye stage measured energy and previously measured energy at the machine, the crystal efficiency change over the course of the day adds a source of normalization error to the calculated neutral densities. To combat the latter issue, periodic energy measurements were taken between NVDF measurements, i.e., approximately every 10 plasma pulses, and the laser was tuned to maximize the output energy.

The upper plot in Fig. 6.7 shows the temporal evolution of the density as a function of power. Each pressure trace is the average of 10 Proto-MPEX pulses (a plasma pulse for each data 
point in a measured NVDF). There is some apparent anti-correlation in Proto-MPEX between rf power and neutral density. In other words, as expected, the neutral density decreases with increasing rf power. The trends observed for the measured absolute neutral densities are in agreement with trends of densities calculated from the measured neutral pressure. ${ }^{1}$ However, it should be mentioned that the neutral pressure displayed by the Baratron pressure gauge is a combination of both molecular and atomic deuterium and therefore the measured neutral density should always be less than the density calculated from the measured pressure. Figure
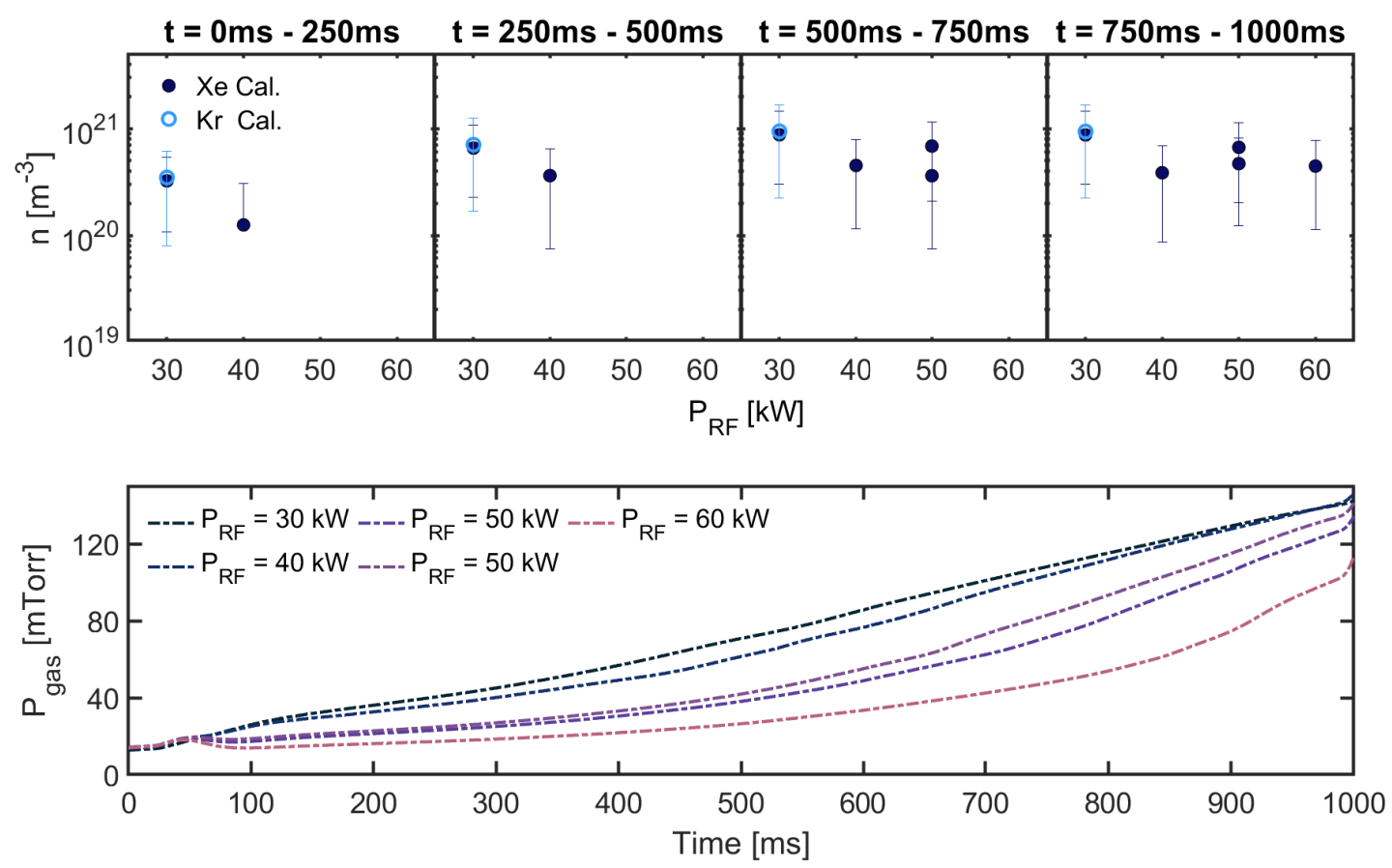

FiguRE 6.7: Evolution of density throughout the plasma pulse as a function of $\mathrm{P}_{\mathrm{RF}}$ is shown in the upper plot. Density was calibrated with both xenon and krypton for $\mathrm{P}_{\mathrm{RF}}=30 \mathrm{~kW}$. Density values derived from krypton are shown in light blue open circles and density values derived from xenon are shown with dark blue solid circles. Below, the neutral pressure evolution over the duration of the plasma pulse (averaged over the plasma pulses used to construct the NVDFs) for each $\mathrm{P}_{\mathrm{RF}}$ is shown.

6.8 shows neutral deuterium temperature as a function of power. Since reliable signal is not observed for early times in the plasma pulse or for large rf powers, the data points for high power and early times are not displayed. Temperature is largely independent of $\mathrm{P}_{\mathrm{RF}}$ for powers below $60 \mathrm{~kW}$. Additionally, the general trend is that the temperatures peak for early times in the plasma pulse and decrease for later times. The decrease in neutral temperature is likely

\footnotetext{
${ }^{1}$ Densities are calculated from the pressure traces using the ideal gas law and the measured neutral temperature.
} 
due to the termination of the plasma for later times. It was observed that the plasma does not persist through the entire rf pulse since the amount of gas being injected smothers the plasma at later times. This effect is more noticeable for lower powers and the plasma persists longer for higher powers. It is during these afterglow times that the greatest signal amplitudes are observed. The neutral temperature plays an important role for processes and efficiency of

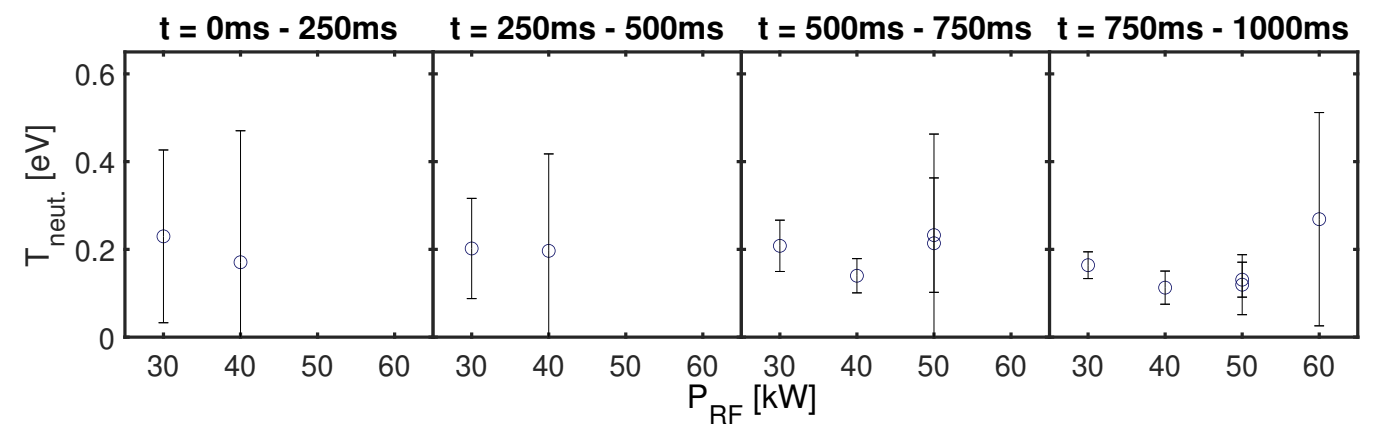

Figure 6.8: Neutral deuterium temperature as a function of power. Temperature derived from fits with sufficient signal are shown. A general decrease in temperature for later times throughout the pulse is observed due to the lack of the persistence of the plasma for higher pressures and later times.

Proto-MPEX. It is generally assumed that when molecular deuterium is dissociated the two atomic deuterium particles are born with equal parts of the dissociation energy $(\sim 4 \mathrm{eV})$ [74]. However, as mentioned previously, this is not in agreement with measurements made by Galante et al. [58] or this work. Additionally, Owen et al simulated neutral molecular temperatures in Proto-MPEX during standard operation near the edge of the plasma column. Along the entire axial length of Proto-MPEX, the neutral molecular temperature is between 0.1 and $0.2 \mathrm{eV}$, in good agreement with the measurements here. Here the neutral temperatures are $\sim 0.2 \mathrm{eV}$. Either these measurements are dominated by neutrals recycled from the chamber walls or are cooled through radiative processes.

Bulk flow of neutrals at $r=2 \mathrm{~cm}$ for different $\mathrm{rf}$ powers are shown in Fig. 6.9. Neutral deuterium bulk flows are measured to flow radially inward. Inward radial flow is expected since the ions are flowing axially away from the source (both upstream and downstream) [66]. As the ions are accelerated towards the targets, a deficit in particles is recovered with neutral particles. As rf power increases, the neutral bulk flow decreases monotonically. Bulk flow remains approximately constant for a unique rf power. 


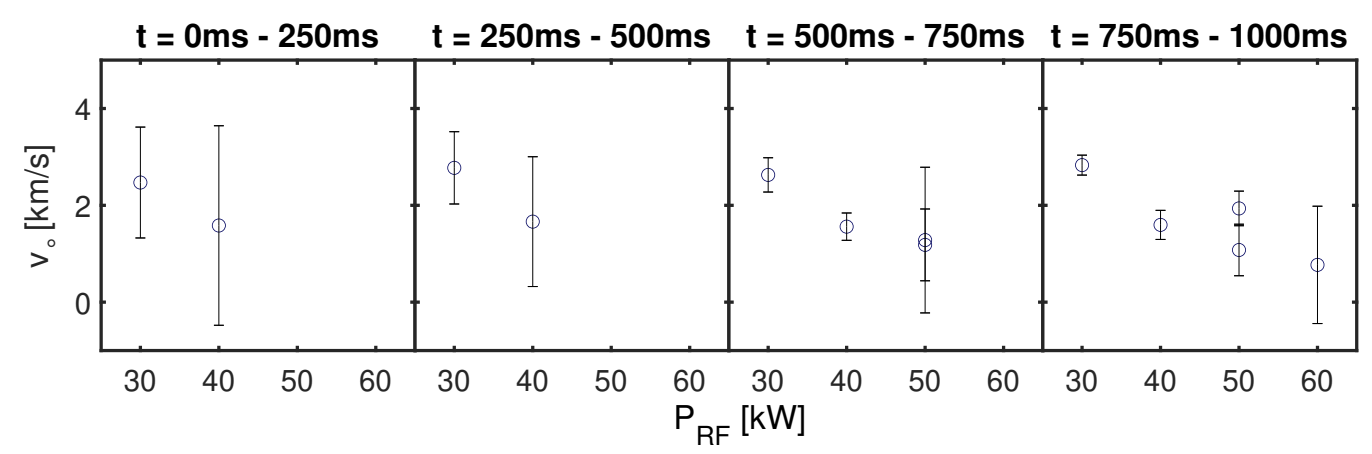

Figure 6.9: Temporal evolution of neutral deuterium bulk flow as a function of power. Bulk flow is observed to be radially inward and decreases monotonically as rf power increases. Bulk flow is measured to be constant in time for a given rf power.

\subsection{Radial Measurements}

To further investigate the neutral deuterium in Proto-MPEX, NVDFs were collected radially upstream from the antenna. It should be noted that measurements are physically restricted by an aluminum magnet spacer between magnets 1 and 2 . As a result, $r=3,4,5 \mathrm{~cm}$ are inaccessible. Figure 6.10 shows the time evolution of deuterium NVDFs for $r=7,6,2,1$, and 0 $\mathrm{cm}\left((\mathrm{a})-(\mathrm{e})\right.$ respectively) for $\mathrm{P}_{\mathrm{RF}}=30 \mathrm{~kW}$. While discernible signal is seen for all times, $0-250$ ms still yields fits with the largest errors. The neutral density measurements were calibrated with both xenon and krypton. Even though the TALIF signal for xenon was much worse than for krypton, the calibrated density values produced by the two methods are in excellent agreement with each other. The data shown in Fig. 6.10 are displayed separately in Figures 6.11 (density versus $r$ ), 6.12 (neutral temperature versus $r$ ), and 6.13 (neutral bulk flow versus $r$ ). In Fig. 6.11, the densities calibrated with both xenon (dark blue solid circles) and krypton (light blue open circles) are shown in the upper plot. Only modest variations in the neutral density are observed radially. All the variations are within the error bars of the measurements. Thus, for these conditions, the neutral atomic deuterium density profile is essentially flat. Magee et al. observed a hollow neutral profile in a helicon krypton plasma [138]. A hollow neutral profile is possibly observed here, but the evidence for such a conclusion is weak. The lower plot shows the pressure as measured by the baratron pressure gauge throughout the plasma pulse. Though they are not distinguishable, there are 5 pressure traces plotted, one for each radial position. The plasma produced by Proto-MPEX was very reproducible for these data, compared with the power variation data where noticeably different pressure profiles were observed for the same conditions. The earliest times in the plasma pulse yield neutral temperatures with significant 


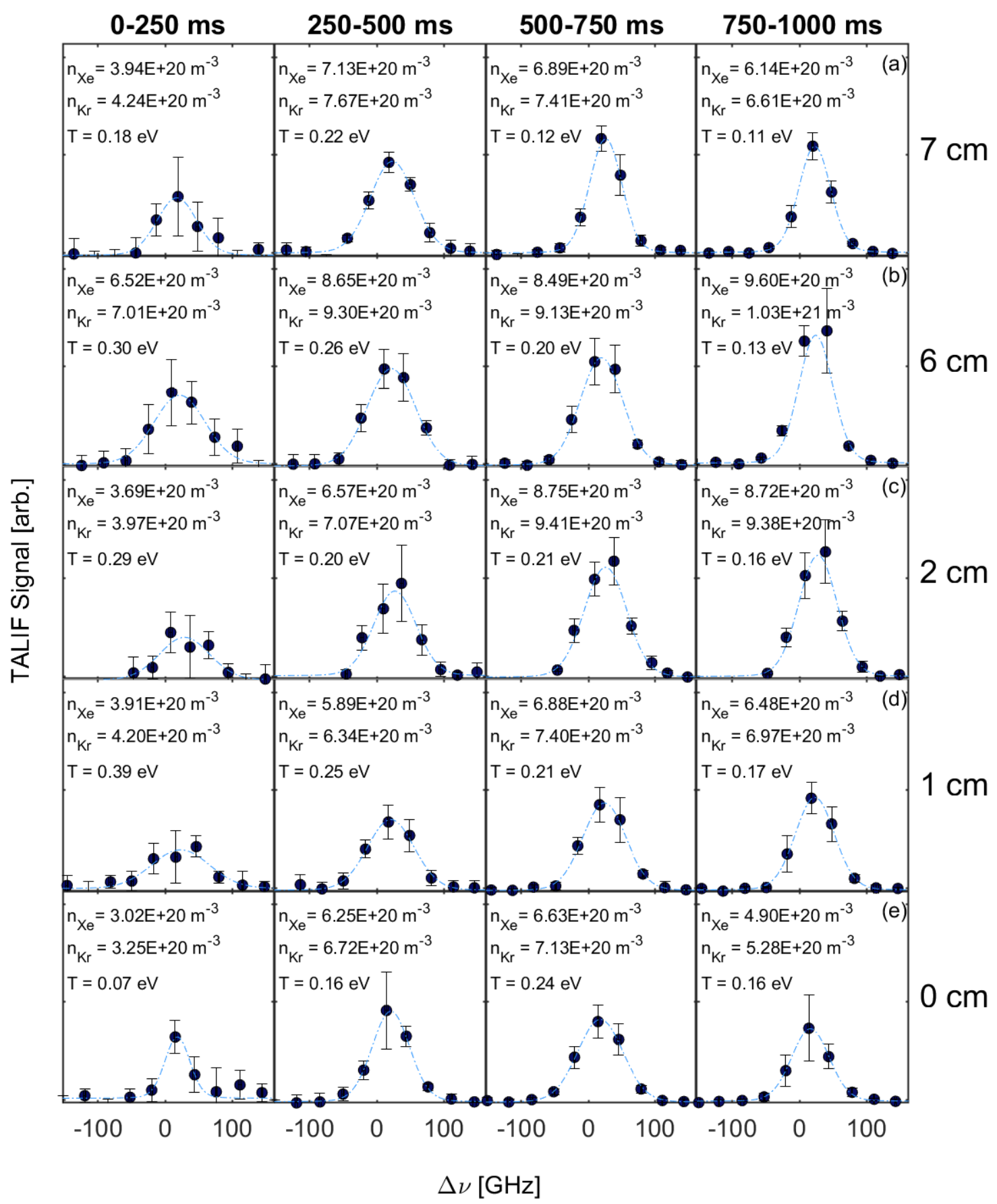

FiguRE 6.10: Time evolution of deuterium NVDFs for $r=7,6,2,1$, and $0 \mathrm{~cm}$ in (a)-(e), respectively, for $\mathrm{P}_{\mathrm{RF}}=30 \mathrm{~kW}$. Earliest times in the plasma pulse produce signal but with low SNR which yields a large spread of values as compared to later times.

variations, likely due to poor SNR in the plasma pulse. Similar to the measured density profile, the temperature profile for each time interval in the plasma pulse are flat within the error. 

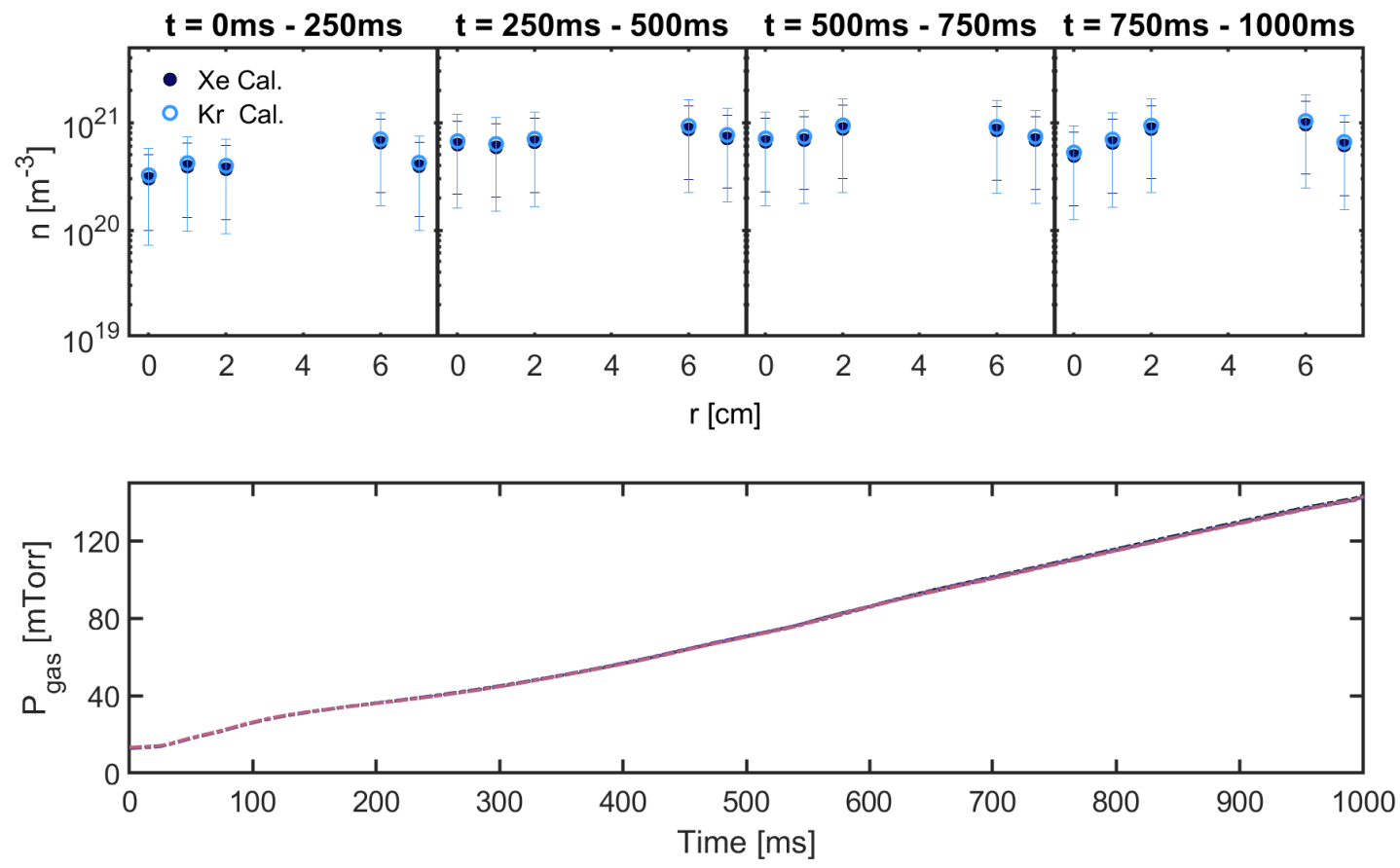

FiguRE 6.11: (upper plot) Density versus $r$ calibrated with both xenon (dark blue solid circles) and krypton (light blue open circles). Within the uncertainty of the measurement, the radial profile is essentially flat. Data for $r=3,4$, and $5 \mathrm{~cm}$ was inaccessible due to a magnetic support rod that physically obstructed the collection apparatus. The lower plot shows the pressure trace throughout the plasma pulse. All pressure traces for each radial location are plotted and it is shown that plasma pressure evolution is very reproducible for these data.

However, the general trend is that the neutral temperature decreases as time increases, as seen in the previous study.

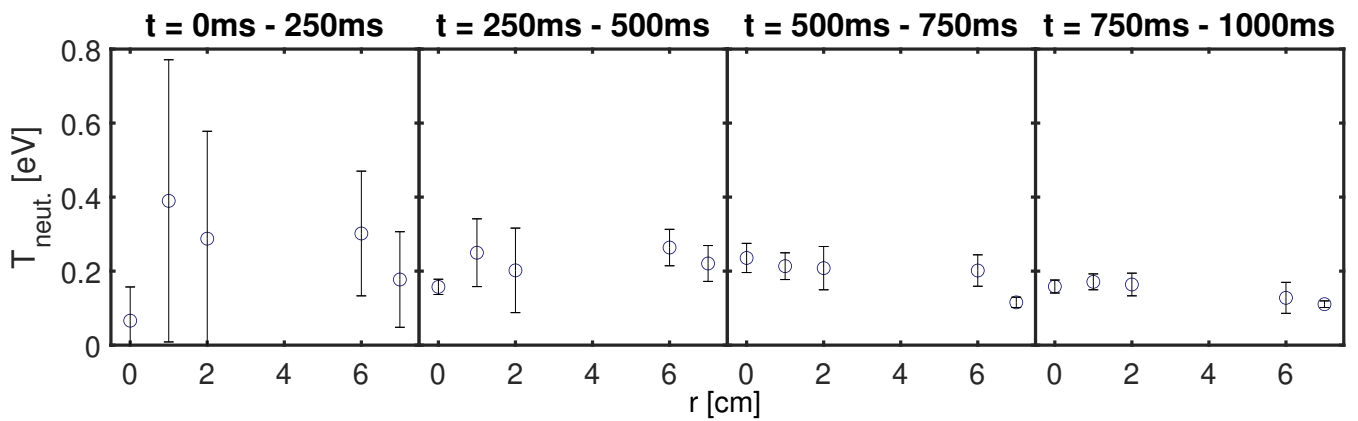

FiguRE 6.12: Temperature versus $r$ throughout the plasma pulse. Earliest times show large variation in neutral temperature that relaxes for later times. The temperature is only weakly dependent on radius, but has a modest dependence on time. As the plasma evolves and pressure increases, the neutral temperature decreases overall.

Shown in Fig. 6.13 are the measured neutral deuterium bulk flows for $30 \mathrm{~kW}$ at different radial locations. Similar to the measurements for different rf powers, the bulk flow at different 


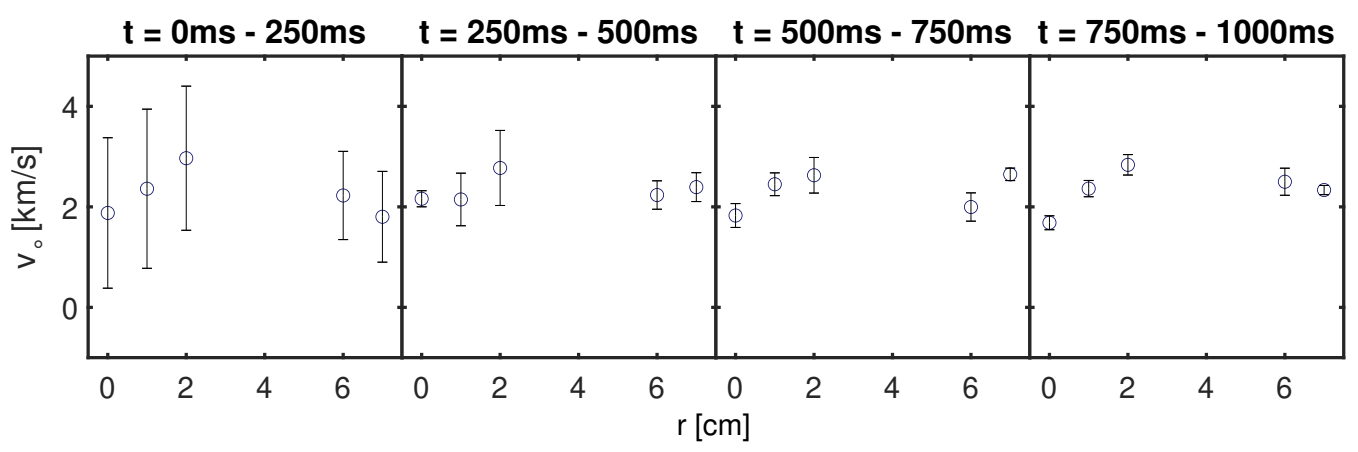

FiguRE 6.13: Neutral bulk flow versus $r$ throughout the plasma pulse. Earliest times show large variation in neutral bulk flow that relaxes for later times. The neutral motion is measured to be radially inward. Neutral bulk flow is constant for a fixed radial location, but increases for increasing radius before asymptoting at the plasma edge $(r=6-7 \mathrm{~cm})$.

radial locations are measured to be radially inward. For unique radial locations, the bulk flow is approximately constant over the plasma pulse. However, the neutral bulk flow increases for increasing radii and remains approximately constant at the plasma edge $(r=6-7 \mathrm{~cm})$. Significant radial flow is observed on axis of Proto-MPEX. Intuitively, radial flow would approach zero for measurements made for decreasing $r$. A possible explanation for the large observed flows on axis is increased absorption along the path of the laser beam. Since the laser beam has a considerably small diameter after the compression stage of the confocal apparatus, the power density of the beam is significantly large for several locations along the beam path and not specifically only at the focal spot. It is likely that the measurements made near the axis of the machine average over a few centimeters. In this case, the observed flow mapped to the axis of the machine is an average flow for radial locations greater than zero centimeters. Furthermore, since greatest ionization is expected on axis of Proto-MPEX, the radial bulk flow measurements may also be weighted to have greater influence from particles off-axis where more absorbers are expected to reside, further increasing the measured flow.

\subsection{Summary}

Initial TALIF measurements on the Proto-MPEX device at ORNL show that it is possible to successfully free-space inject UV light for TALIF from over $20 \mathrm{~m}$ away from the device. This is especially important for the future of diagnosing fusion devices where laser systems must be placed a significant distance from the device. Neutral deuterium absolute density, temperature and bulk flow dependence on rf power and radial spatial location were successfully obtained. 
Twenty laser pulses are recorded for a single Proto-MPEX plasma pulse. A deuterium NVDF is constructed from ten Proto-MPEX plasma pulses. The time evolution of deuterium TALIF signal is constructed by averaging over every five laser pulses, yielding a time resolution of 250 ms. Measured absolute densities were calibrated using both xenon and krypton and derived values are in excellent agreement with each other. Density profiles exhibit a weak inverse dependence on rf power and are essentially flat for all radial locations and are nominally observed to be on the order of $10^{20} \mathrm{~m}^{-3}$.

Neutral deuterium temperatures are measured to be approximately $0.2 \mathrm{eV}$, significantly lower than predicted values for dissociated neutral hydrogen [74]. However, the measured neutral temperature is consistent with measurements made by Galante and are in good agreement with predicted molecular neutral temperatures in the edge of Proto-MPEX [58, 137]. Neutral deuterium temperatures showed a slight decrease for later times in the plasma pulse and generally exhibit flat profiles.

Measured deuterium bulk flows are radially inward for all radial locations and $\mathrm{rf}$ powers at velocities of $\sim 2 \mathrm{~km} / \mathrm{s}$. Radial inward flow of neutrals is expected to replenish a deficit of particles are axis due to axial ion flow. Neutral flow exhibits no variation in time for a unique radial location and rf power. Neutral bulk flow decreases for increasing rf power and increases for increasing radii until the plasma edge $(r=6-7 \mathrm{~cm})$ where the flow remains approximately constant. 


\section{Chapter 7}

\section{Discussion and Future Work}

\subsection{II LIF and Applications to Ion Thrusters}

The main focus of this portion of this work is systems that use iodine as a propellant, specifically HTs. These measurements demonstrate that ion flow and external magnetic field strengths can be determined non-perturbatively using LIF in iodine plasmas. These are the first measurements of an iodine ion velocity distribution function and the first measurements of the hyperfine structure and Zeeman splitting of the iodine ion transition identified by Hargus et al.[22] A confocal collection setup provides a way to measure plasma quantities in regions with limited optical access.

Iodine LIF signal is inversely proportional to iodine partial pressure inside the custom reference cell. Internal pressure is controlled by adjusting coolant temperature that is circulated through a chilling finger on the cell. Ample SNR for I II LIF measurements is observed for pressure between $\sim 10$ and $\sim 50$ mTorr $\left(\mathrm{T}_{\mathrm{CF}} \sim-10-+5^{\circ} \mathrm{C}\right)$. Significant hyperfine structure was measured for this particular transition. Hyperfine structure depends on the magnetic dipole and electric quadrupole coupling coefficients $A$ and $B$, respectively. These coupling coefficients were not able to be determined in this work because individual hyperfine peaks were not resolvable. A Monte-Carlo fitting analysis was used to estimate the most-probable ion temperature of $0.09 \pm 0.01 \mathrm{eV}$ for these measurements and is in agreement with ion temperatures in similar experiments [130]. The rest wavelength for the transition probed here was measured to be $696.0694 \mathrm{~nm}$, which serves as the zero-flow reference for iodine thruster measurements. 
A technique to measure the external magnetic field strength in a HT from the Zeeman-split spectrum was presented. The separation of the $\sigma$-peaks are directly proportional to the magnetic field strength. A Zeeman-splitting coefficient of $\epsilon_{z}=1.9 \pm 0.1 \mathrm{GHz} / \mathrm{kG}$ was calculated and a minimum magnetic field strength of $\sim 1 \mathrm{kG}$ was able to be measured from I II Zeemansplit spectra. To measure typical magnetic field strengths in HTs, a more complex optical configuration is needed. Theoretical minimum magnetic field strength using a polarizer $-\lambda / 4$ waveplate combination is $\sim 100 \mathrm{G}$ and is sufficient to measure typical magnetic field strengths in HTs [136].

Recently, a portable LIF system was developed at WVU to diagnose argon neutrals and iodine ions. This new portable LIF system will allow the LIF sequence pioneered in this work to be applied to a wide range of systems at remote facilities. Figure 7.1 shows a velocity distribution function measured with this new system. This measurement is in excellent agreement with the measurements made with the Matisse laser system and was measured at significantly less power $(\sim 3 \mathrm{~mW})$. The lineshape measured with the portable diode system also shows significant hyperfine structure.

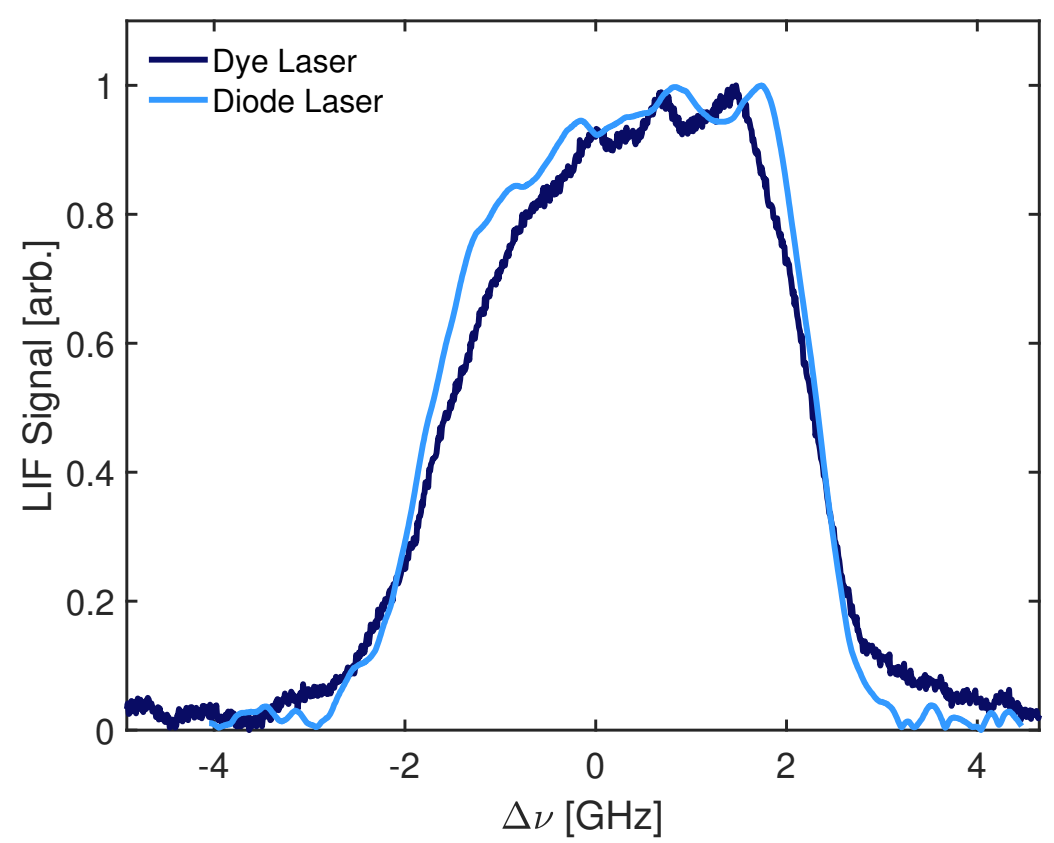

FiguRE 7.1: Measured I II lineshape measured with a new portable LIF system using a diode laser system. The measured lineshape exhibits significant hyperfine structure and is in excellent agreement with previous measurements using the Matisse laser system.

Further characterization of the hyperfine structure will be attempted using the the diode laser system. The diode laser system has a highly repeatable scan range and LIF signal can be 
collected continuously and averaged to reveal fine features. Doppler-free techniques are likely to reveal the hyperfine structure, as suggested by Fig. 5.8. Additionally, the Doppler-free lineshape can be measured with an appropriate IR laser. Using an IR laser centered approximately around $1393 \mathrm{~nm}$, a two-photon measurement can be made that probes the same transition investigated here. Injecting the IR light in counter-propagating directions, the Doppler motion of the particle of the particle does not affect absorption since the motion of the particle is effectively canceled out by the absorption of two photons traveling in opposite directions. This technique has been used to measure line broadening effects that are negligible compared to Doppler-broadening [139].

A full understanding of a Hall thruster plume using iodine as a propellant requires knowledge of neutral and ionized molecular and neutral atomic iodine compositions in the exhaust. Plume composition is listed as a major open-ended goal of Ref. [21]. To this end, a final focus is to identify and diagnose transitions for each species listed above. Neutral molecular iodine transitions are well documented [140] and already have identified transitions in wavelength ranges of current LIF setups at WVU.

\subsection{Deuterium TALIF and Applications to Fusion-relevant Plas- mas}

\subsubsection{SOLPS Simulations of Proto-MPEX}

Plasma transport in Proto-MPEX has been simulated by using software called SOLPS to predict plasma and neutral dynamics during typical Proto-MPEX operation [66, 137, 141]. SOLPS is a multi-fluid plasma solver software that incorporates a kinetic Monte-Carlo neutral code [142]. Owen et al. compared double-Langmuir probe data to simulated values to validate SOLPS results. Of particular interest to this work are the neutral predictions during standard ProtoMPEX operation. A hollow neutral profile is predicted at the helicon window with nearly a three order of magnitude increase in neutral density at the location of TALIF measurements. Predicted atomic neutral density at the TALIF measurement location from Ref. [137] is $10^{17}$ $10^{18} \mathrm{~m}^{-3}$. More recently, Showers et al. presented additional SOLPS modeling of Proto-MPEX [141]. The configuration of Proto-MPEX in Ref. [141] is similar to the configuration of ProtoMPEX during TALIF experiments. Neutral atomic deuterium density in the region of TALIF 
measurements is predicted to be $\sim 10^{19} \mathrm{~m}^{-3}$. Figure 7.2 shows the predicted neutral deuterium profiles for typical Proto-MPEX operation from (a) Ref. [137] and (b) [141]. In both plots, a gray vertical line indicates the location of TALIF measurements.
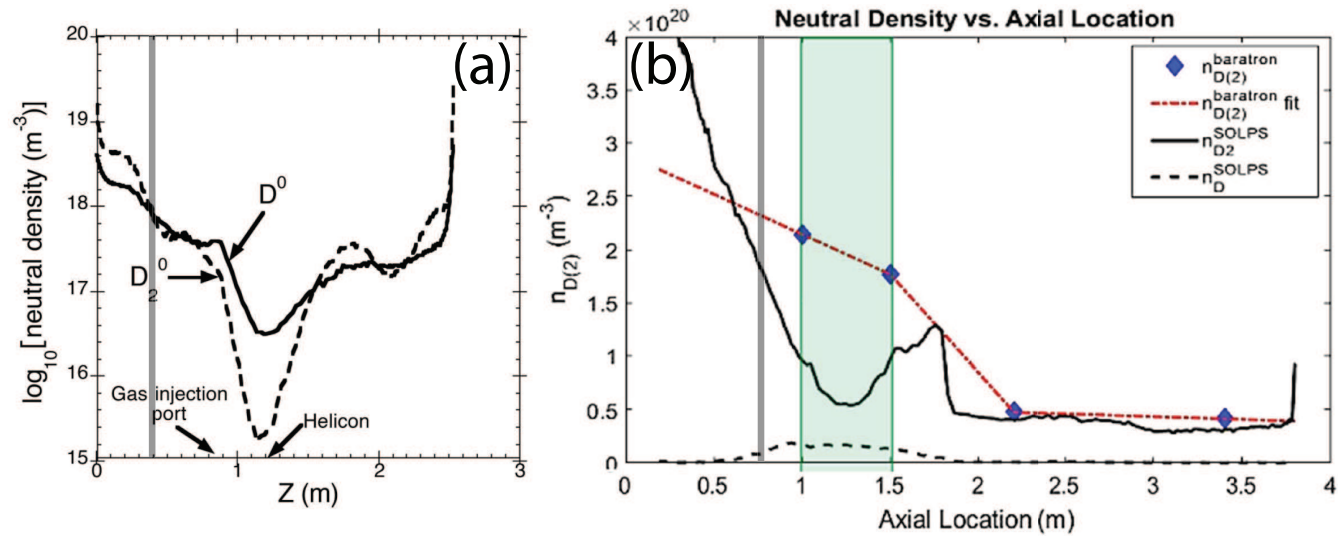

Figure 7.2: Neutral density profiles for deuterium during typical Proto-MPEX operation from (a) Ref. [137] and (b) from Ref. [141]. In figure (a), the solid black curve indicates predicted atomic neutral deuterium density. Atomic neutral deuterium density is indicated by the black dashed line in figure (b). For both plots, the gray vertical line indicates the region of TALIF measurements. Figures adapted from Refs. [137 $]^{1}$ and $[141]^{2}$

Owen et al. also used SOLPS to predict the neutral molecular temperature at the plasma column edge. The temperature of the chamber wall was fixed at $0.1 \mathrm{eV}$ for these simulations. Figure 7.3 shows the neutral molecular deuterium temperature at the edge of the plasma for typical Proto-MPEX operation. Diatomic neutral temperature is predicted to be slightly higher than the wall temperature $(0.1-0.2 \mathrm{eV})$. Comparison of these predictions and measured values are discussed later in this chapter.

\subsubsection{TALIF Discussion and Future Work}

Several challenges were initially encountered in implementing TALIF on Proto-MPEX. Significant electromagnetic fields are produced by the plasma source. Therefore, the laser system and TALIF electronics were located far from Proto-MPEX, as will be the case for TALIF systems on future fusion and PMI plasma devices. In this work, UV laser pulse transmission over $\sim 20 \mathrm{~m}$ to the vacuum vessel was demonstrated. Significant energy losses were observed over this distance due to losses in optical materials and beamline absorption, but enough energy was delivered to the target plasma to excite ground state deuterium.

\footnotetext{
${ }^{1}$ Reproduced from Ref [137], with permission of AIP publishing.

$2 @$ IOP Publishing. Reproduced with permission. All right reserved.
} 


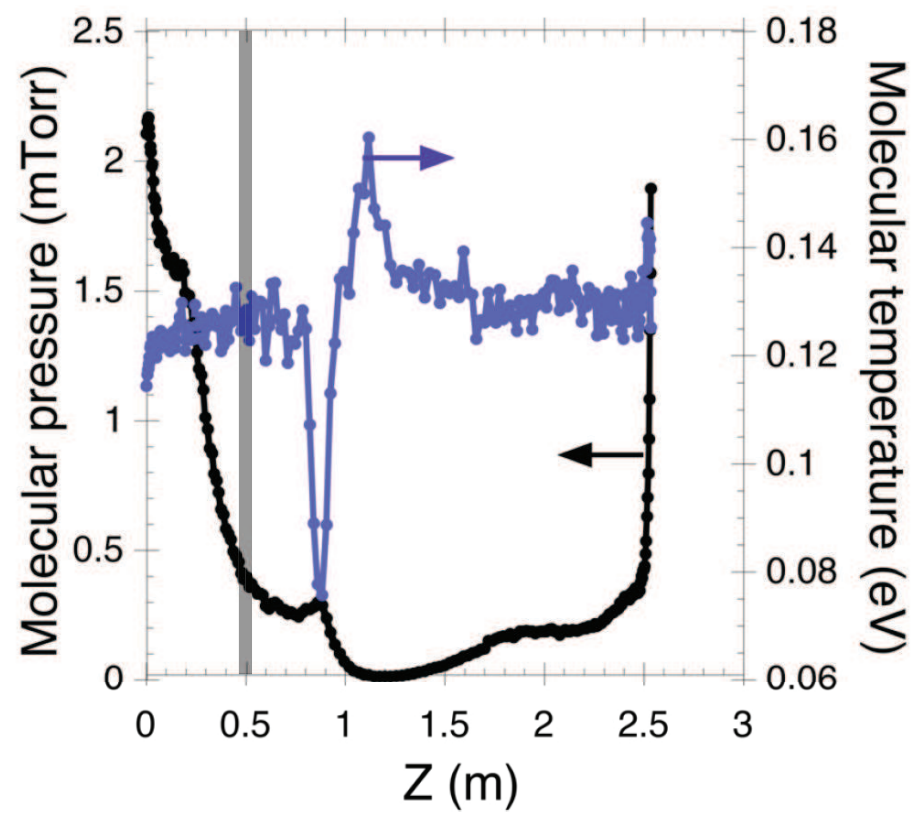

FiguRE 7.3: Molecular neutral deuterium predicted temperature profile from SOLPS. Neutral temperature is shown by the blue curve with temperature values listed on the right vertical axis. A solid gray vertical line indicates the location of TALIF measurements. Neutral temperatures are predicted to be slightly above the chamber wall temperature of $0.1 \mathrm{eV}$. Reproduced from Ref [137], with permission of AIP publishing.

Neutral deuterium VDFs were measured at different radial locations and rf powers. Absolute neutral deuterium densities were calibrated with both xenon and krypton and derived values are in excellent agreement. Neutral densities decrease for increasing rf powers and is weakly observed to increase throughout the plasma pulse, consistent with increasing gas pressure. Absolute neutral densities on the order of $10^{20} \mathrm{~m}^{-3}$. Increased gas pressures were required compared to normal Proto-MPEX operation for these measurements. Deuterium densities are predicted to be on the order of $10^{18}-10^{19} \mathrm{~m}^{-3}$ for normal Proto-MPEX operation, where typical measured gas pressures are $\sim 2$ mTorr $[137,141]$. The measured absolute densities measured here are consistent with predicted densities when extrapolated to the operating pressures measured in this work ${ }^{3}$.

Neutral deuterium temperatures are $\sim 0.2 \mathrm{eV}$ for the measurements reported here. Neutral temperatures show weak dependence on radial position, becoming slightly colder for increasing radius. Neutral temperature increases for increasing rf power, as expected. Additionally, neutral temperature was observed to relax to lower temperatures at later times in the pulse, consistent with the plasma termination for later times in the plasma pulse due to large gas pressures.

\footnotetext{
${ }^{3}$ At the end of the measured pressure trace the pressure is $\sim 140$ mTorr which corresponds to densities on the order of $10^{21} \mathrm{~m}^{-3}$.
} 
Measured neutral temperatures agree with predicted neutral molecular temperatures near the edge of the plasma column in Ref. [137]. The molecular temperature is shown to relax to approximately the wall temperature for these simulations. Measured neutral temperatures are modestly larger than predicted, but are measured further from the edge of the plasma column where larger neutral temperatures are expected.

Measured neutral bulk flow is radially inward for all radial locations and powers and was approximately constant throughout the plasma pulse for a unique radial position and rf power combination. Neutral bulk flow decreased for increasing rf powers for measurements reported here. However, neutral bulk flow showed a proportional dependence on radial location until the plasma edge $(6-7 \mathrm{~cm})$ where it remained approximately constant. Radial inward flow of neutrals is expected since axial flow of ions is observed for typical operation [66]. As a deficit in ions is created due to axial acceleration, the neutral particles meet the deficit by flowing in.

It is likely that TALIF systems will need to placed even farther than $20 \mathrm{~m}$ for future fusion devices and, as a result, if TALIF is to be a viable diagnostic, more initial energy-per-pulse will be needed to overcome transmission losses. Current dye-crystal conversion lasers consistently generate $\sim 10 \mathrm{~mJ} / \mathrm{pls}$. for identical pump energies. Such pulse energies are a factor of 2 increase over the highest laser output pulse energies in this work and a factor of 5 increase over the output pulse energies used for these measurements. While an increase in UV pulse energy to compensate for losses over long distances is achievable, addition to the beam line length introduces new alignment challenges. Additionally, beam divergence for a longer beam line will be significant and collimation optics would likely need to be placed periodically throughout the beamline without introducing additional significant losses.

There is a possible alternative multi-photon method that could eliminate many of the transmission issues. Using three photons that sum to the energy needed to excite ground state neutral deuterium shifts the laser wavelength closer to the visible spectrum. Laser wavelengths for 3pLIF $(\sim 307 \mathrm{~nm})$ are easily transmitted by cheaper and more common materials (as compared to $\sim 205 \mathrm{~nm}$ wavelengths). It is also more likely that these wavelengths can be coupled into spectral fibers for long distance transmission. Laser pulse energies are also greater for such wavelengths since one less frequency conversion is required. The ability to fiber couple the injected light eliminates the need for permanent beamlines, extensive optics, and site-of-injection 
optical alignment. Therefore, efforts are underway at WVU to attempt 3pLIF in krypton first (Sec. 2.4.2) with the intent to later successfully collect 3pLIF ground state deuterium signal in fusion-relevant devices. 


\section{Appendix A}

\section{Convolution of Gaussian and}

\section{Lorentzian Lineshapes}

\section{A.1 Convolution of Identical Gaussian Functions}

\section{A.1.1 Relations}

Fourier Transform definition:

$$
\mathcal{F}(f(\nu))=\frac{1}{\sqrt{2 \pi}} \int_{-\infty}^{\infty} d \nu f(\nu) \exp [-i t \nu]=F(t)
$$

Inverse Fourier Transform definition:

$$
\mathcal{F}^{-1}(F(t))=\frac{1}{\sqrt{2 \pi}} \int_{-\infty}^{\infty} d k F(t) \exp [i t \nu]=f(\nu)
$$

Fourier Transform shift property for functions with non-zero centers:

$$
\mathcal{F}\left(f\left(\nu \pm \nu_{\circ}\right)\right)=F(t) \exp \left[ \pm i t \nu_{\circ}\right]
$$

Inverse Fourier Transform shift property for functions with non-zero centers:

$$
\mathcal{F}^{-1}\left(F\left(t \pm t_{\circ}\right)\right)=f(\nu) \exp \left[\mp i t_{\circ} \nu\right]
$$




\section{A.1.2 Derivation}

Begin with the definition for the convolution of two functions:

$$
h\left(\nu-\nu_{\circ}\right)=f\left(\nu-\nu_{\circ}\right) * f\left(\nu-\nu_{\circ}\right)
$$

Equation A.5 is solved by inverse Fourier transforming the product of the Fourier transforms of $f\left(\nu-\nu_{\circ}\right):$

$$
h\left(\nu-\nu_{\mathrm{o}}\right)=\mathcal{F}^{-1}\left(F\left(t-t_{\mathrm{o}}\right) \cdot F\left(t-t_{\mathrm{o}}\right)\right) .
$$

The Fourier transform of $f\left(\nu-\nu_{\circ}\right)$ is calculated by considering a function with zero offset and then invoke Eq. A.3:

$$
\begin{aligned}
\mathcal{F}(f(\nu)) & =\frac{1}{\sqrt{2 \pi}} \int_{-\infty}^{\infty} d \nu \exp \left[-\frac{\nu^{2}}{2 \sigma^{2}}\right] \exp [-i t \nu] \\
& =\frac{1}{\sqrt{2 \pi}} \int_{-\infty}^{\infty} d \nu \exp [-\underbrace{\left(\frac{\nu^{2}}{2 \sigma^{2}}+i t \nu\right)}] .
\end{aligned}
$$

To solve this integral, the expression underbraced is transformed by completing the square:

$$
\frac{\nu^{2}}{2 \sigma^{2}}+i t \nu=\left[\frac{\nu}{\sqrt{2} \sigma}+\frac{i t}{2 / \sqrt{2} \sigma}\right]^{2}+\frac{t^{2}}{4 / 2 \sigma^{2}} .
$$

Plug this expression into the integral:

$$
\begin{aligned}
\mathcal{F}(f(\nu)) & =\frac{1}{\sqrt{2 \pi}} \int_{-\infty}^{\infty} d \nu \exp \left[-\left\{\left(\frac{\nu}{\sqrt{2} \sigma}+\frac{i t}{2 / \sqrt{2} \sigma}\right)^{2}+\frac{t^{2}}{4 / 2 \sigma^{2}}\right\}\right] \\
& =\frac{1}{\sqrt{2 \pi}} \int_{-\infty}^{\infty} d \nu \exp \left[-\left(\frac{\nu}{\sqrt{2} \sigma}+\frac{i t}{2 / \sqrt{2} \sigma}\right)^{2}\right] \exp \left[-\frac{t^{2}}{4 / 2 \sigma^{2}}\right] \\
& =\frac{1}{\sqrt{2 \pi}} \exp \left[-\frac{t^{2}}{4 / 2 \sigma^{2}}\right] \int_{-\infty}^{\infty} d \nu \exp \left[-\left(\frac{\nu}{\sqrt{2} \sigma}+\frac{i t}{2 / \sqrt{2} \sigma}\right)^{2}\right] .
\end{aligned}
$$


A simple substitution yields

$$
\begin{aligned}
\mathcal{F}(f(\nu)) & =\frac{\sigma}{\sqrt{\pi}} \exp \left[-\frac{t^{2}}{4 / 2 \sigma^{2}}\right] \underbrace{\int_{-\infty}^{\infty} d u \exp \left[-u^{2}\right]}_{\sqrt{\pi}} \\
& =\sigma \exp \left[-\frac{t^{2}}{4 / 2 \sigma^{2}}\right] .
\end{aligned}
$$

Therefore,

$$
F(t) \propto \exp \left[-\frac{t^{2}}{4 / 2 \sigma^{2}}\right]
$$

Using Eq. A.3, the F.T. with a non-zero offset is

$$
\begin{aligned}
\mathcal{F}\left(f\left(\nu-\nu_{\circ}\right)\right) & =F(t) \cdot \exp \left[-i t \nu_{\circ}\right] \\
& \propto \exp \left[-\frac{t^{2}}{4 / 2 \sigma^{2}}\right] \cdot \exp \left[-i t \nu_{\circ}\right] \\
& \propto \exp [-\underbrace{\left(\frac{t^{2}}{4 / 2 \sigma^{2}}+i t \nu_{\circ}\right)}_{\text {complete the square }}] \\
& \propto \exp \left[-\left(\frac{\left[t+\frac{i \nu_{\circ}}{\sigma^{2}}\right]^{2}}{4 / 2 \sigma^{2}}+\frac{\nu_{\circ}^{2}}{2 \sigma^{2}}\right)\right] \\
& \propto \exp \left[-\frac{\nu_{\circ}^{2}}{2 \sigma^{2}}\right] \exp \left[-\frac{\left[t+t_{\circ}\right]^{2}}{4 / 2 \sigma^{2}}\right] \\
& \propto \exp \left[-\frac{\left[t+t_{\mathrm{o}}\right]^{2}}{4 / 2 \sigma^{2}}\right],
\end{aligned}
$$

where $t_{\circ}=i \nu_{\circ} / \sigma^{2}$.

The Fourier transform of $h$ is

$$
\begin{aligned}
H\left(t+t_{\circ}\right) & =F\left(t+t_{\circ}\right) \cdot F\left(t+t_{\circ}\right) \\
& \propto \exp \left[-\frac{\left[t+t_{\mathrm{o}}\right]^{2}}{2 / 2 \sigma^{2}}\right] .
\end{aligned}
$$


The inverse F.T. is now calculated. A function with no offset is inverse Fourier transformed first.

$$
\begin{aligned}
\mathcal{F}^{-1}(H(t)) & \propto \frac{1}{\sqrt{2 \pi}} \int_{-\infty}^{\infty} d t \exp \left[-\frac{t^{2}}{2 / 2 \sigma^{2}}\right] \exp [i t \nu] \\
& \propto \frac{1}{\sqrt{2 \pi}} \int_{-\infty}^{\infty} d t \exp [-\underbrace{\left(\frac{t^{2}}{2 / 2 \sigma^{2}}-i t \nu\right)}_{\text {complete the square }}] \\
& \propto \frac{1}{\sqrt{2 \pi}} \int_{-\infty}^{\infty} d t \exp \left[-\left(t \sigma-\frac{i \nu}{2 \sigma}\right)^{2}\right] \exp \left[-\frac{\nu^{2}}{2\left(2 \sigma^{2}\right)}\right] \\
& \propto \frac{1}{\sqrt{2 \pi}} \exp \left[-\frac{\nu^{2}}{2\left(2 \sigma^{2}\right)}\right] \int_{-\infty}^{\infty} d t \exp \left[-\left(t \sigma-\frac{i \nu}{2 \sigma}\right)^{2}\right]
\end{aligned}
$$

A simple substitution solves the integral:

$$
\begin{aligned}
\mathcal{F}^{-1}(H(t)) & \propto \frac{1}{\sqrt{2 \pi}} \exp \left[-\frac{\nu^{2}}{2\left(2 \sigma^{2}\right)}\right] \int_{-\infty}^{\infty} \frac{1}{\sigma} d u \exp \left[-u^{2}\right] \\
& \propto \frac{1}{\sigma \sqrt{2 \pi}} \exp \left[-\frac{\nu^{2}}{2\left(2 \sigma^{2}\right)}\right] \underbrace{\int_{-\infty}^{\infty} d u \exp \left[-u^{2}\right]}_{\sqrt{\pi}} \\
& \propto \frac{1}{\sigma \sqrt{2}} \exp \left[-\frac{\nu^{2}}{2\left(2 \sigma^{2}\right)}\right] \\
& \propto \exp \left[-\frac{\nu^{2}}{2\left(2 \sigma^{2}\right)}\right]=f(\nu) .
\end{aligned}
$$


Lastly, using Eq. A.4, the convolved function is calculated to be:

$$
\begin{aligned}
h\left(\nu-\nu_{\circ}\right) & =f(\nu) \exp \left(-i t_{\circ} \nu\right) \\
& \propto \exp \left[-\frac{\nu^{2}}{2\left(2 \sigma^{2}\right)}\right] \exp \left[\frac{\nu_{\circ}}{\sigma^{2}} \nu\right] \\
& \propto \exp [-\frac{1}{2\left(2 \sigma^{2}\right)} \underbrace{\left(\nu^{2}-\frac{\nu_{\circ}}{\sigma^{2}} \nu\right)}_{\text {complete the square }}] \\
& \propto \exp \left(-\frac{\left(\nu-2 \nu_{\circ}\right)^{2}}{2\left(2 \sigma^{2}\right)}+\nu_{\circ}^{2}\right) \\
& \propto \exp \left(\nu_{\circ}^{2}\right) \exp \left(-\frac{\left(\nu-2 \nu_{\circ}\right)^{2}}{2\left(2 \sigma^{2}\right)}\right) \\
& \propto \exp \left(-\frac{\left(\nu-2 \nu_{\circ}\right)^{2}}{2\left(2 \sigma^{2}\right)}\right) \\
& \propto \exp \left(-\frac{\left(\nu-2 \nu_{\circ}\right)^{2}}{2 \gamma^{2}}\right) .
\end{aligned}
$$

Therefore, the convolution of two identical Gaussian functions with mean $\nu_{\circ}$ and width $\sigma$ yields a new Gaussian with mean equal to $2 \nu_{\circ}$ and width $\gamma=\sqrt{2} \sigma$.

\section{A.2 Convolution of Identical Lorentzian Functions}

\section{A.2.1 Derivation}

The convolution of two identical Lorentzian functions is given by

$$
f_{\mathrm{L}}(\nu) * f_{\mathrm{L}}(\nu)=\int_{-\infty}^{\infty} d \tau f_{\mathrm{L}}(\tau) f_{\mathrm{L}}(\nu-\tau)
$$

and is calculated in a similar way to the convolution of two identical Gaussian functions. First the Fourier transform of the Lorentzian is calculated:

$$
\begin{aligned}
\mathcal{F}\left[f_{\mathrm{L}}(\nu)\right] & =\frac{1}{2 \pi} \int_{-\infty}^{\infty} d \nu \frac{\Gamma}{\nu^{2}+\Gamma^{2}} \exp (-i \nu t) \\
& =\frac{1}{\pi} \int_{0}^{\infty} d \nu \frac{\Gamma}{\nu^{2}+\Gamma^{2}} \cos (\nu t)-\frac{1}{2 \pi} \int_{-\infty}^{\infty} d \nu \frac{\Gamma}{\nu^{2}+\Gamma^{2}} i \sin (\nu t) \\
& =\exp (-\Gamma t) .
\end{aligned}
$$


The product of two Fourier transformed Lorentzian functions is

$$
\begin{aligned}
\mathcal{F}\left[f_{\mathrm{L}}(\nu)\right] \cdot \mathcal{F}\left[f_{\mathrm{L}}(\nu)\right] & =\exp (-\Gamma t) \cdot \exp (-\Gamma t) \\
& =\exp (-(\Gamma+\Gamma) t) \\
& =\exp (-\xi t) .
\end{aligned}
$$

The result of the previous expression is identical to the Fourier transform of a Lorentzian function with HWHM $\xi=\Gamma+\Gamma$. Therefore the inverse Fourier transform of this expression is the final result:

$$
\mathcal{F}^{-1}\left[\mathcal{F}\left[f_{\mathrm{L}}(\nu)\right] \cdot \mathcal{F}\left[f_{\mathrm{L}}(\nu)\right]\right]=\frac{\xi}{\nu^{2}+\xi^{2}}
$$

thus showing that the convolution of two identical Lorentzian functions produces another Lorentzian function with a resultant width that is a simple sum of the input Lorentzian functions. That is, $\xi=\Gamma+\Gamma$.

\footnotetext{
${ }^{1}$ This only true if $t$ and $\Gamma$ are positive.
} 


\section{Appendix B}

\section{Matisse Alignment Procedure}

Day-to-day minor alignment of the Matisse laser system is typical. Before any alignment, the Matisse laser system should have warmed up for nominally one hour. At times less than one hour, the laser may be unstable. If needed, the procedure for a full cavity alignment can be found in Ref. [88]. Steps for power optimization are:

1. Confirm dye pressure is at desired value. Note: it is common for the dye pressure to drop over the warm up period.

2. Adjust vertical (M1V) and horizontal (M1H) position of the output coupler (M1) shown by the green arrows in Fig. B.1. Best practice is to start with either the vertical or horizontal adjustment first, make small adjustments and wait for the power measurement to stabilize and then make another adjustment. It is easy to pass the knob position that produces maximum laser power during this alignment step. To return to an alignment of maximum power, simply rotate the adjustment knob in the opposite direction until maximum is observed again. Note there is some hysteresis in the adjustment. Once a maximum power has been determined repeat this step for the other adjustment, that is, vertical or horizontal.

3. Iteratively repeat the previous step until any adjustment of M1 results in a decrease of power. 
4. Adjust vertical (TM4V) and horizontal (TM4H) position of the tuning mirror (M4) shown by the green arrows in Fig. B.2. Best practice is to start with either the vertical or horizontal adjustment first, make small adjustments and wait for the power measurement to stabilize and then make another adjustment. It is easy to pass the knob position that produces maximum laser power during this alignment step. To return to an alignment of maximum power, simply rotate the adjustment knob in the opposite direction until maximum is observed again. Note there is some hysteresis in the adjustment. Once a maximum power has been determined repeat this step for the other adjustment, that is, vertical or horizontal. ${ }^{1}$

5. Iteratively repeat the previous step until any adjustment of M1 results in a decrease of power.

6. Perform a BiFi scan and move motor position to maximum of the red curve that produces roughly the desired wavelength.

7. Perform a thin etalon scan and move the motor position slightly left of a minimum of the blue curve that produces the desired wavelength.

8. Check the power. If power is sufficient, the alignment is complete. If not, proceed to next step.

9. Carefully remove the laser cavity lid to reveal the thick etalon.

10. Adjust vertical and horizontal position of the thick etalon shown by the green arrows in Fig. B.3. Best practice is to start with either the vertical or horizontal adjustment first, make small adjustments and wait for the power measurement to stabilize and then make another adjustment. It is easy to pass the knob position that produces maximum laser power during this alignment step. To return to an alignment of maximum power, simply rotate the adjustment knob in the opposite direction until maximum is observed again. Note there is some hysteresis in the adjustment. Once a maximum power has been determined repeat this step for the other adjustment, that is, vertical or horizontal.

11. Repeat steps $2-8$.

\footnotetext{
${ }^{1}$ The adjustments for this mirror are more sensitive than M1.
} 
The above described procedure may require several iterations. If power is still insufficent, consider preparing a new batch of dye. If new dye and power optimization does not provide sufficient power, the cavity may require a re-alignment.

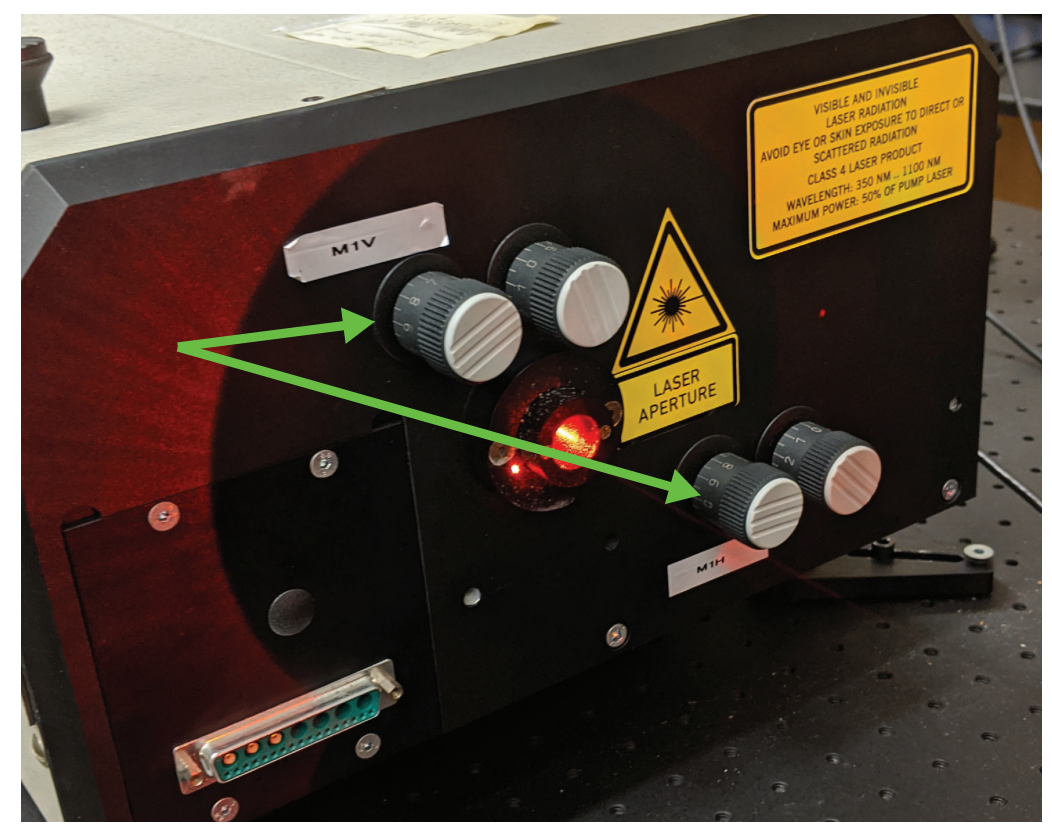

Figure B.1: Vertical and Horizontal adjustment knobs for the output couple (M1).

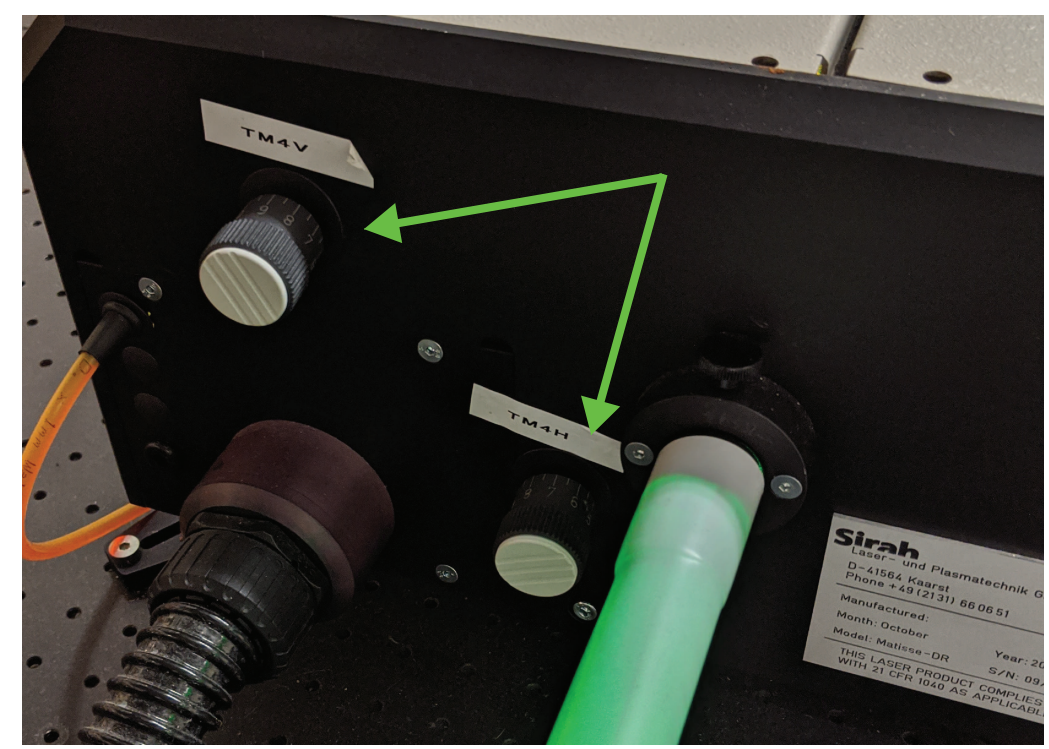

FiguRE B.2: Vertical and Horizontal adjustment knobs for the tuning mirror (M4). 


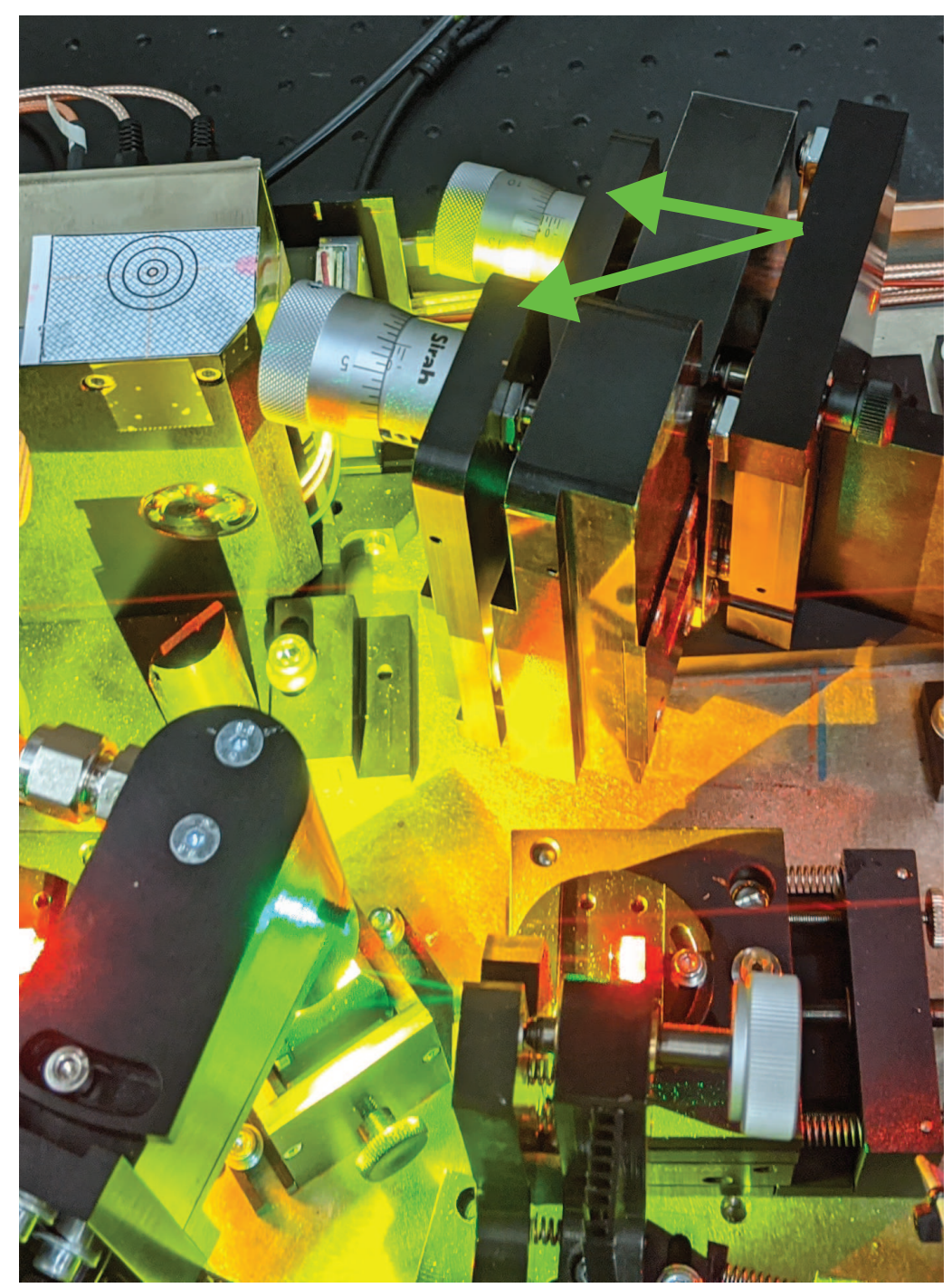

FiguRE B.3: Vertical and Horizontal adjustment knobs for the thick etalon. 


\section{Appendix C}

\section{Dye Recipes}

\section{C.1 Matisse Pyridine 1 (LDS698) Recipe (I II LIF)}

Two variants of this recipe were employed in this work. Traditionally, pyridine 1 is dissolved in a $9: 1$ mixture of ethylene glycol (EG):ethylene glycol monophenyl ether (EPH) at a concentration of 1.07 grams of dye for every liter of solution. The dye pump used for the Matisse holds $4 \mathrm{~L}$ of solution. The traditional recipe is as follows:

1. Measure out $4.28 \mathrm{~g}$ of pyridine 1 (the dye).

2. Combine $400 \mathrm{~mL}$ of (EPH) to $3600 \mathrm{~mL}$ of (EG) (the solution).

3. Mix the dye and the solution via a magnetic stirrer for a minimum of $\sim 2$ hours with foil over top the dye-solution mixture to prevent debris from falling in the dye. ${ }^{1}$

A variant was used that shows modest dye peak shift towards wavelengths used to probe iodine. The dye recipe is nearly identical to the traditional except the solution does not contain any EPH. Therefore, the recipe is simply:

1. Measure out $4.28 \mathrm{~g}$ of pyridine 1.

2. Mix the dye with $4 \mathrm{~L}$ of $\mathrm{EG}$ for a minimum of $\sim 2$ hours.

\footnotetext{
${ }^{1}$ It is standard to let the mixture mix overnight, though if this timescale isn't optimal 2 hours will usually suffice. The main goal is to ensure that there are not undissolved clumps of dye still in the solution before returning the solution to the dye pump.
} 


\section{C.2 Sirah Cobra Stretch Rhodamine Dye Recipe (D I, Xe I, Kr I TALIF)}

The dye mixture used for the TALIF laser system is considerably more complex than what is used for the Matisse. The recipe requires a mixture of two different types of dyes and is a two stage mixing process since on dye cell requires a different concentration. The procedure is as follows:

1. Measure out $0.153 \mathrm{~g}$ of rhodamine B (rhodamine 610 ) and $0.036 \mathrm{~g}$ of rhodamine 101 (rhodamine 640).

2. Combine dye with $900 \mathrm{~mL}$ of 200 proof non-denatured ethyl alcohol (ethanol) ${ }^{2}$.

3. Let dye-solution mixture mix for $\sim 2$ hours via magnetic stirrer. ${ }^{3}$

4. Collect 700 of the $900 \mathrm{~mL}$ solution and pour it into the pre-amplification dye cell dye pump, saving the last $200 \mathrm{~mL}$ for the next step.

5. Add $1400 \mathrm{~mL}$ of 200 proof ethanol to leftover $200 \mathrm{~mL}$ mixture from the previous step.

6. Pour the diluted $1600 \mathrm{~mL}$ mixture into the amplifier dye cell cye pump.

\footnotetext{
${ }^{2} 200$ proof methanol can also be used.

${ }^{3}$ Contrary to the recipe for the Matisse laser system, the user should adhere to shorter mix times (i.e., $\sim 2$ hours) since ethanol tends to evaporate faster than EG and therefore the concentration the mixture will be incorrect.
} 


\section{Appendix D}

\section{Energy Conversion Curves for}

\section{TALIF Measurements}

Energy conversions curves for each set of data taken for across wavelengths needed for each species were made. A new conversion curve was made for each dye change. First measurements did not use krypton as a calibration gas and therefore there is no conversion curve for krypton for the July 18, 2019 measurements.

\section{D.1 Deuterium}

For measurements corresponding to 18 July 2019
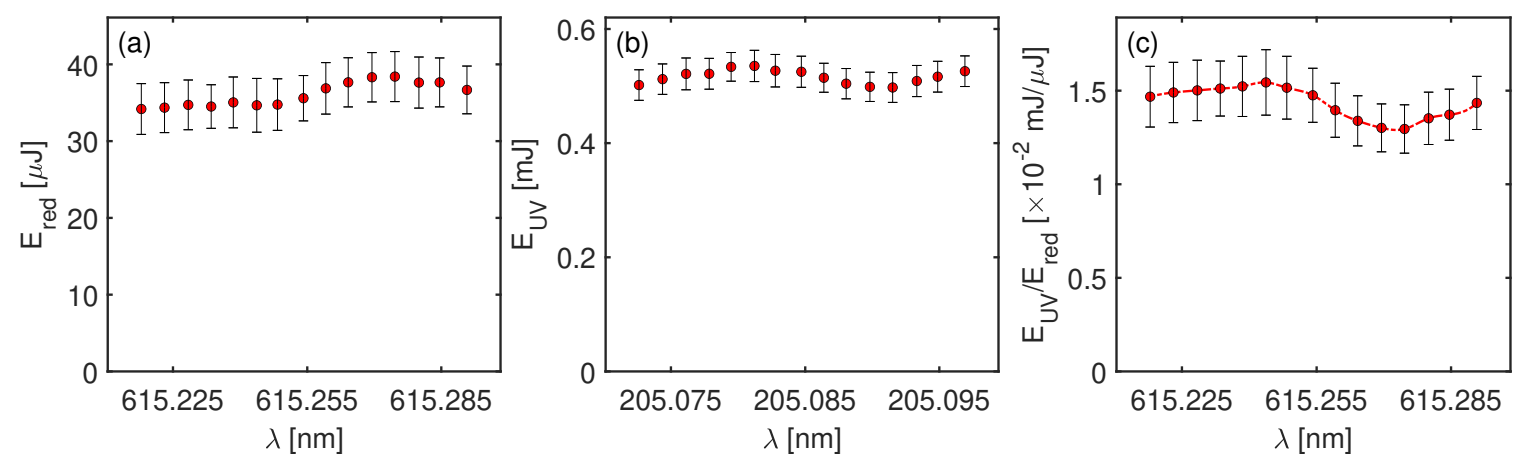

Figure D.1: (a) Average energy-per-pulse versus wavelength at dye stage monitored by the wavelength meter, (b) average energy-per-pulse versus wavelength at Proto-MPEX monitored by the Gentec energy meter, and (c) conversion curve between the two as a function of wavelength. 
For measurements corresponding to 22 July 2019
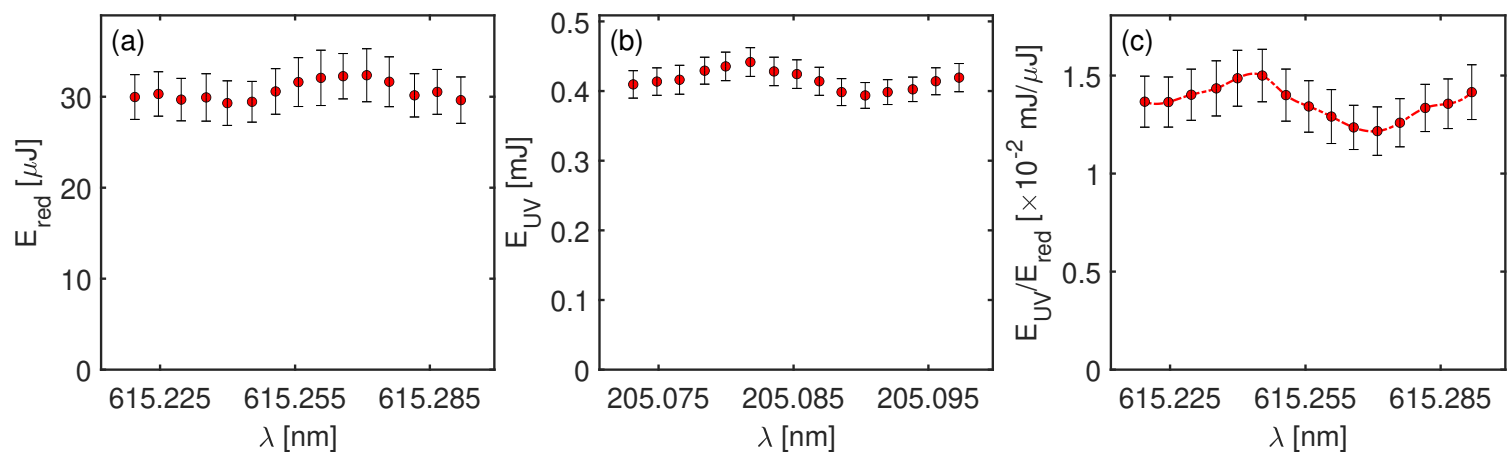

FiguRE D.2: (a) Average energy-per-pulse versus wavelength at dye stage monitored by the wavelength meter, (b) average energy-per-pulse versus wavelength at Proto-MPEX monitored by the Gentec energy meter, and (c) conversion curve between the two as a function of wavelength.

\section{D.2 Xenon}

For measurements corresponding to 18 July 2019
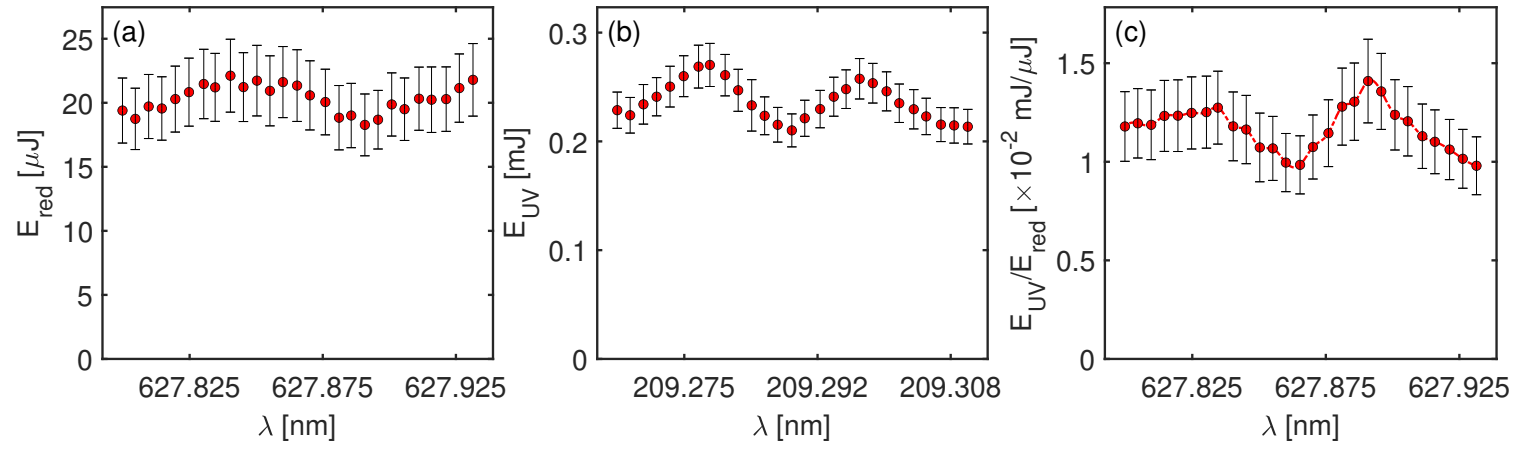

Figure D.3: (a) Average energy-per-pulse versus wavelength at dye stage monitored by the wavelength meter, (b) average energy-per-pulse versus wavelength at Proto-MPEX monitored by the Gentec energy meter, and (c) conversion curve between the two as a function of wavelength. 
For measurements corresponding to 22 July 2019
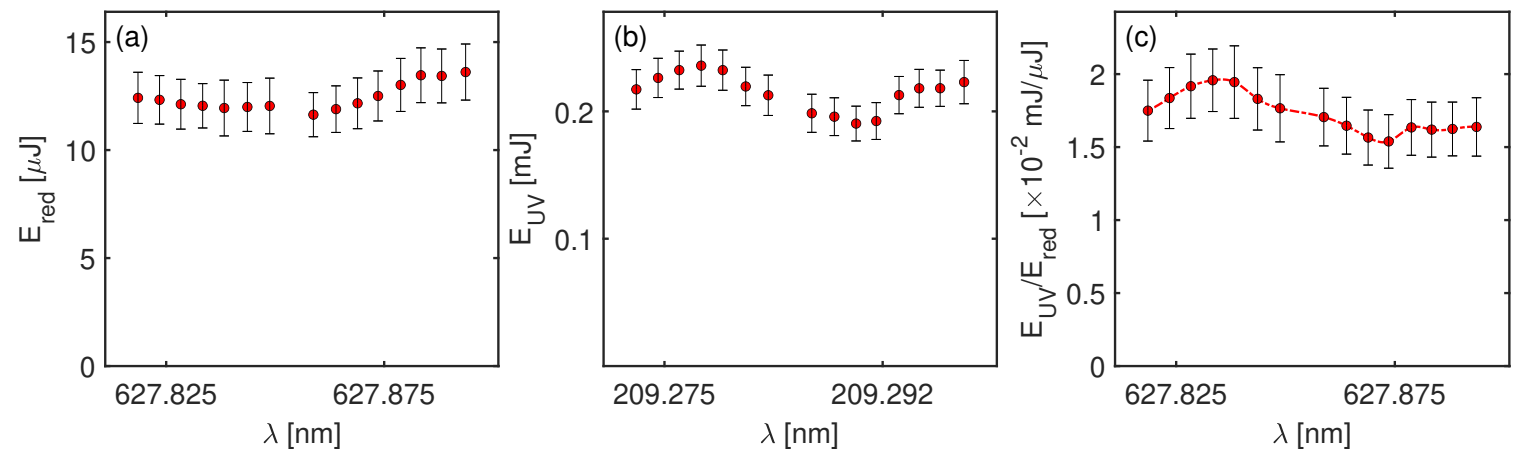

FiguRE D.4: (a) Average energy-per-pulse versus wavelength at dye stage monitored by the wavelength meter, (b) average energy-per-pulse versus wavelength at Proto-MPEX monitored by the Gentec energy meter, and (c) conversion curve between the two as a function of wavelength.

\section{D.3 Krypton}

For measurements corresponding to 22 July 2019
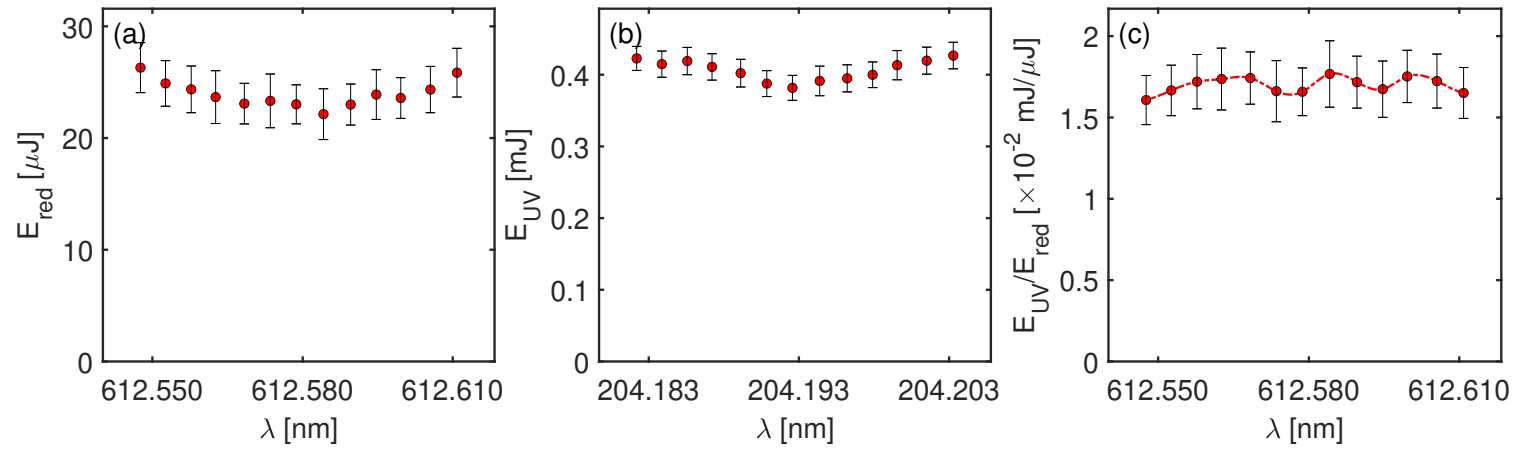

FiguRE D.5: (a) Average energy-per-pulse versus wavelength at dye stage monitored by the wavelength meter, (b) average energy-per-pulse versus wavelength at Proto-MPEX monitored by the Gentec energy meter, and (c) conversion curve between the two as a function of wavelength. 


\section{Appendix E}

\section{Hamamatsu Data}

E.1 Gain

\begin{tabular}{|lcccccc|}
\hline \hline Control Voltage (V) & 0.4 & 0.5 & 0.6 & 0.7 & 0.8 & 0.9 \\
Gain & $6.5 \times 10^{3}$ & $4.1 \times 10^{4}$ & $1.9 \times 10^{5}$ & $6.6 \times 10^{5}$ & $2.0 \times 10^{6}$ & $4.7 \times 10^{7}$ \\
\hline
\end{tabular}




\section{E.2 Quantum Efficiency}

\begin{tabular}{|c|c|c|c|c|c|}
\hline Wavelength & Cathode & Quantum & Wavelength & Cathode & Quantum \\
\hline$(\mathrm{nm})$ & $\begin{array}{c}\text { Radiant } \\
\text { Sensitivity } \\
(\mathrm{mA} / \mathrm{W})\end{array}$ & Efficiency & $(\mathrm{nm})$ & $\begin{array}{c}\text { Radiant } \\
\text { Sensitivity } \\
(\mathrm{mA} / \mathrm{W})\end{array}$ & Efficiency \\
\hline 230 & 1.18 & 0.64 & 610 & 77.1 & 15.7 \\
\hline 240 & 2.51 & 1.30 & 620 & 77.5 & 15.5 \\
\hline 250 & 5.60 & 2.78 & 630 & 77.8 & 15.3 \\
\hline 260 & 8.95 & 4.27 & 640 & 77.7 & 15.1 \\
\hline 270 & 11.2 & 5.14 & 650 & 77.6 & 14.8 \\
\hline 280 & 12.6 & 5.67 & 660 & 77.4 & 14.5 \\
\hline 290 & 13.6 & 5.82 & 670 & 76.6 & 14.2 \\
\hline 300 & 14.4 & 5.95 & 680 & 75.6 & 13.8 \\
\hline 310 & 15.1 & 6.04 & 690 & 74.1 & 13.3 \\
\hline 320 & 15.9 & 6.16 & 700 & 72.1 & 12.8 \\
\hline 330 & 16.8 & 6.31 & 710 & 69.5 & 12.1 \\
\hline 340 & 17.8 & 6.49 & 720 & 66.5 & 11.5 \\
\hline 350 & 18.8 & 6.66 & 730 & 64.0 & 10.9 \\
\hline 360 & 20.0 & 6.89 & 740 & 61.7 & 10.3 \\
\hline 370 & 21.2 & 7.11 & 750 & 59.6 & 9.85 \\
\hline 380 & 22.7 & 7.41 & 760 & 57.4 & 9.37 \\
\hline 390 & 24.9 & 7.92 & 770 & 55.2 & 8.89 \\
\hline 400 & 28.7 & 8.90 & 780 & 53.1 & 8.44 \\
\hline 410 & 33.3 & 10.1 & 790 & 50.7 & 7.96 \\
\hline 420 & 38.3 & 11.3 & 800 & 47.8 & 7.41 \\
\hline 430 & 43.5 & 12.5 & 810 & 45.1 & 6.90 \\
\hline 440 & 49.0 & 13.8 & 820 & 41.5 & 6.28 \\
\hline 450 & 56.4 & 15.5 & 830 & 37.10 & 5.54 \\
\hline 460 & 62.4 & 16.8 & 840 & 30.00 & 4.43 \\
\hline 470 & 66.7 & 17.6 & 850 & 21.71 & 3.17 \\
\hline 480 & 70.2 & 18.1 & 860 & 12.90 & 1.86 \\
\hline 490 & 72.2 & 18.3 & 870 & 6.43 & 0.92 \\
\hline 500 & 73.6 & 18.3 & 880 & 2.81 & 0.40 \\
\hline 510 & 74.4 & 18.1 & 890 & 1.17 & 0.16 \\
\hline 520 & 74.8 & 17.8 & 900 & 0.51 & 0.07 \\
\hline 530 & 74.9 & 17.5 & 910 & 0.23 & 0.03 \\
\hline 540 & 75.0 & 17.2 & 920 & 0.11 & 0.02 \\
\hline 550 & 75.2 & 17.0 & & & \\
\hline 560 & 75.3 & 16.7 & & & \\
\hline 570 & 75.8 & 16.5 & & & \\
\hline 580 & 75.9 & 16.2 & & & \\
\hline 590 & 76.4 & 16.1 & & & \\
\hline 600 & 76.7 & 15.9 & & & \\
\hline
\end{tabular}




\section{Bibliography}

${ }^{1}$ F. Chen, "Introduction to plasma physics and controlled fusion vol. 1: plasma physics," in, 2nd ed. (Springer, New York, 1984), pp. 1-16.

${ }^{2}$ D. S. Thompson, R. Khaziev, M. Fortney-Henriquez, S. Keniley, E. E. Scime, and D. Curreli, "Three-dimensional cross-field flows at the plasma-material interface in an oblique magnetic field," Physics of Plasmas 27, 073511 (2020).

${ }^{3}$ K. Holste, P. Dietz, S. Scharmann, K. Keil, T. Henning, D. Zschtzsch, M. Reitemeyer, B. Nauschtt, F. Kiefer, F. Kunze, J. Zorn, C. Heiliger, N. Joshi, U. Probst, R. Thringer, C. Volkmar, D. Packan, S. Peterschmitt, K. T. Brinkmann, H.-G. Zaunick, M. H. Thoma, M. Kretschmer, H. J. Leiter, S. Schippers, K. Hannemann, and P. J. Klar, "Ion thrusters for electric propulsion: scientific issues developing a niche technology into a game changer," Review of Scientific Instruments 91, 061101 (2020).

${ }^{4}$ R. Goddard, "A method of reaching extreme altitudes," Nature 105, 809-811 (1920).

${ }^{5}$ E. Y. Choueiri, "A critical history of electric propulsion: the first 50 years (1906-1956)," Journal of Propulsion and Power 20, 193-203 (2004).

${ }^{6} \mathrm{D}$. Thompson, "Three-dimensional multispecies distribution functions in a plasma boundary with an oblique magnetic field," PhD thesis (West Virginia University, 2018).

${ }^{7}$ D. Yongjie, X Yu, P. Wuji, W. Liqiu, Hongbo, S. Hezhi, L. Peng, L. Hong, and Y. Daren, "A type of cylindrical hall thruster with a magnetically insulated anode," Journal of Physics D: Applied Physics 50, 145203 (2017).

${ }^{8}$ J.-P. Boeuf, "Tutorial: physics and modeling of hall thrusters," Journal of Applied Physics 121, 011101 (2017). 
${ }^{9}$ E. Y. Choueiri, "Fundamental difference between the two hall thruster variants," Physics of Plasmas 8, 5025-5033 (2001).

${ }^{10}$ D. M. Goebel and I. Katz, "Hall thrusters," in Fundamentals of electric propulsion, 1st ed. (John Wiley and Sons, Ltd, 2008) Chap. 7, pp. 325-392.

${ }^{11}$ S. Mazouffre, D. Gawron, V. Kulaev, J. P. Luna, and N. Sadeghi, "A laser spectroscopic study on xe+ ion transport phenomena in the $\mathrm{E} \times \mathrm{B}$ discharge of a hall effect thruster," AIP Conference Proceedings 993, 447-454 (2008).

${ }^{12}$ W. A. Hargus and M. Cappelli, "Laser-induced fluorescence measurements of velocity within a hall discharge," Applied Physics B 72, 961-969 (2001).

${ }^{13}$ R. Dressler, Y.-H. Chiu, and D. Levandier, "Propellant alternatives for ion and hall effect thrusters," in 38th aerospace sciences meeting and exhibit (January 2000).

${ }^{14}$ D. Jacobson and H. Manzella David, "50 kw class krypton thruster performance," in 39th aiaa/asme/sae/asee joint propulsion conference and exhibit. (July 2003).

${ }^{15}$ S. Grishin, V. Erofeev, A. Zharinov, V. Naumkin, and I. Safronov, "Characteristics of the two-step ion accelerator with an anode layer," Zh Prikl Mekh Tekh Fiz, 28-36 (1978).

${ }^{16}$ J. Szabo, B. Pote, S. Paintal, M. Robin, A. Hiller, R. D. Branam, and R. E. Huffman, "Performance evaluation of an iodine-vapor hall thruster," Journal of Propulsion and Power 28, 848-857 (2012).

${ }^{17}$ O. Tverdokhlebov and A. Semenkin, "Iodine propellant for electric propellant-to be or not to be," in 37th aiaa/asme/sae/asee joint propulsion conference and exhibit. (2001).

${ }^{18}$ A. Kieckhafer and L. B. King, "Energetics of propellant options for high-power hall thrusters," Journal of Propulsion and Power 23, 21-26 (2007).

${ }^{19}$ M. Hopkins, "Evaluation of magnesium as a hall thruster propellant," PhD thesis (Michigan Technological University, 2014).

${ }^{20}$ T. Steinberger and E. Scime, "Laser-induced fluorescence of singly ionized iodine," Journal of Propulsion and Power 34, 1235-1239 (2018).

${ }^{21}$ A. Hiller, "Revolutionizing space propulsion through the characterization of iodine as fuel for hall-effect thrusters," MA thesis (Air Force Institute of Technology, Wright Patterson Air Force Base, 2011). 
${ }^{22}$ W. Hargus, J. Lubkeman, K. Remy, and A. Gonzales, "Investigation of singly ionized iodine spectroscopy in support of electrostatic propulsion diagnostics development," in 48th aiaa/asme/sae/asee joint propulsion conference and exhibit. (July 2012).

${ }^{23}$ M. Choi, "Modeling an iodine hall thruster plume in the iodine satellite (isat)," in (2016).

${ }^{24}$ C. Huang and L. Li, "Magnetic confinement fusion: a brief review," Frontiers in Energy 12, 305-313 (2018).

${ }^{25} \mathrm{~S}$. Jacquemot, "Inertial confinement fusion for energy: overview of the ongoing experimental, theoretical and numerical studies," Nuclear Fusion 57, 102024 (2017).

${ }^{26}$ J. Linke, J. Du, T. Loewenhoff, G. Pintsuk, B. Spilker, I. Steudel, and M. Wirtz, "Challenges for plasma-facing components in nuclear fusion," Matter and Radiation at Extremes 4, 056201 (2019).

${ }^{27}$ J. B. O. Caughman, R. H. Goulding, T. M. Biewer, T. S. Bigelow, I. H. Campbell, J. Caneses, S. J. Diem, A. Fadnek, D. T. Fehling, R. C. Isler, E. H. Martin, C. M. Parish, J. Rapp, K. Wang, C. J. Beers, D. Donovan, N. Kafle, H. B. Ray, G. C. Shaw, and M. A. Showers, "Plasma source development for fusion-relevant material testing," Journal of Vacuum Science \& Technology A 35, 03E114 (2017).

${ }^{28} \mathrm{M}$. Showers, "A power accounting analysis of the proto-mpex linear device and target heat flux extrapolations to mpex-based on proto-mpex discharges," https://trace.tennessee.edu/utk_graddiss/5436, $\mathrm{PhD}$ thesis (University of Tennessee, 2019).

${ }^{29}$ Y. Ueda, K. Schmid, M. Balden, J. Coenen, T. Loewenhoff, A. Ito, A. Hasegawa, C. Hardie, M. Porton, and M. Gilbert, "Baseline high heat flux and plasma facing materials for fusion," Nuclear Fusion 57, 092006 (2017).

${ }^{30} \mathrm{M}$. Galante, "Two-photon absorption laser induced fluorescence measurements of neutral density in helicon plasma," PhD thesis (West Virginia University, West Virginia University Libraries, 2013).

${ }^{31}$ R. Hazeltine, M. Calvin, P. Valanju, and E. Solano, "Analytical calculation of neutral transport and its effect on ions," Nuclear Fusion 32, 3-14 (1992). 
${ }^{32}$ R. L. Boivin, J. A. Goetz, A. E. Hubbard, J. W. Hughes, I. H. Hutchinson, J. H. Irby, B. LaBombard, E. S. Marmar, D. Mossessian, C. S. Pitcher, J. L. Terry, B. A. Carreras, and L. W. Owen, "Effects of neutral particles on edge dynamics in alcator c-mod plasmas," Physics of Plasmas 7, 1919-1926 (2000).

${ }^{33}$ F. Wagner, G. Becker, K. Behringer, D. Campbell, A. Eberhagen, W. Engelhardt, G. Fussmann, O. Gehre, J. Gernhardt, G. v. Gierke, G. Haas, M. Huang, F. Karger, M. Keilhacker, O. Klüber, M. Kornherr, K. Lackner, G. Lisitano, G. G. Lister, H. M. Mayer, D. Meisel, E. R. Müller, H. Murmann, H. Niedermeyer, W. Poschenrieder, H. Rapp, H. Röhr, F. Schneider, G. Siller, E. Speth, A. Stäbler, K. H. Steuer, G. Venus, O. Vollmer, and Z. Yü, "Regime of improved confinement and high beta in neutral-beam-heated divertor discharges of the asdex tokamak," Phys. Rev. Lett. 49, 1408-1412 (1982).

${ }^{34}$ A. Team, "The h-mode of ASDEX," Nuclear Fusion 29, 1959-2040 (1989).

${ }^{35}$ A. E. Hubbard, "Physics and scaling of the h-mode pedestal," Plasma Physics and Controlled Fusion 42, A15-A35 (2000).

${ }^{36} \mathrm{~J}$. W. Connor and H. R. Wilson, "A review of theories of the l-h transition," Plasma Physics and Controlled Fusion 42, R1-R74 (1999).

${ }^{37}$ L. Chen, G. Xu, A. Nielsen, W. Gao, Y. Duan, H. Liu, L. Wang, M. Li, M. Wang, X. Zhang, R. Chen, H. Wang, Z. Sun, S. Ding, N. Yan, S. Liu, L. Shao, W. Zhang, G. Hu, J. Li, L. Zhang, and B. Wan, "Study on the $\mathrm{l}-\mathrm{h}$ transition power threshold with RF heating and lithium-wall coating on EAST," Nuclear Fusion 56, 056013 (2016).

${ }^{38}$ P. Snyder, J. Hughes, T. Osborne, C. Paz-Soldan, W. Solomon, M. Knolker, D. Eldon, T. Evans, T. Golfinopoulos, B. Grierson, R. Groebner, A. Hubbard, E. Kolemen, B. LaBombard, F. Laggner, O. Meneghini, S. Mordijck, T. Petrie, S. Scott, H. Wang, H. Wilson, and Y. Zhu, "High fusion performance in super h-mode experiments on alcator c-mod and DIII-d," Nuclear Fusion 59, 086017 (2019).

${ }^{39}$ E. Marmar, "Recycling processes in tokamaks," Journal of Nuclear Materials 76-77, 59-67 (1978).

${ }^{40}$ J Winter, "Tokamak wall coatings," Physics of Plasmas 36, B263-B276 (1994). 
${ }^{41}$ J. H. Yu, M. J. Baldwin, M. J. Simmonds, and A. Zalonik, "Time-resolved laser-induced desorption spectroscopy (lids) for quantified in-situ hydrogen isotope retention measurement and removal from plasma facing materials," Review of Scientific Instruments 90, 073502 (2019).

${ }^{42}$ United States. Department of Energy. Office of Science, DOESC (USDOE Office of Science (SC) (United States)), and Office of Fusion Energy Sciences (FES), "Research needs for magnetic fusion energy sciences. report of the research needs workshop (renew) bethesda, maryland, june 8-12, 2009.," in (United States. Dept. of Energy. Office of Science; Distributed by the Office of Scientific and Technical Information, U.S. Dept. of Energy, 2009), pp. 86-87.

${ }^{43}$ R. Magee, M. Galante, D. McCarren, E. Scime, R. Boivin, N. Brooks, R. Groebner, D. Hill, and G. Porter, "A two-photon absorption laser induced fluorescence diagnostic for fusion plasmas," Review of Scientific Instruments 83, 10D701 (2012).

${ }^{44} \mathrm{X} . \mathrm{Zhu}$ and K. Pu, "A simple collisional-radiative model for low-pressure argon discharges," Journal of Physics D: Applied Physics 40, 2533-2538 (2007).

${ }^{45}$ J. Franek, S. Nogami, V Demidov, M. Koepke, and E. Barnat, "Correlating metastable-atom density, reduced electric field, and electron energy distribution in the post-transient stage of a 1-torr argon discharge," Plasma Sources Science and Technology 24, 034009 (2015).

${ }^{46}$ N. Kafle, T. Biewer, and D. Donovan, "Dual-pass upgrade to the thomson scattering diagnostic on the prototype-material plasma exposure experiment (proto-mpex)," Review of Scientific Instruments 89, 10C107 (2018).

${ }^{47}$ A. O'Keefe and D. Deacon, "Cavity ringdown optical spectrometer for absorption measurements using pulsed laser sources," Review of Scientific Instruments 59, 2544-2551 (1988).

${ }^{48} \mathrm{D}$ Body and B. Chadwick, "Simultaneous elemental analysis system using laser induced breakdown spectroscopy," Review of Scientific Instruments 72, 1625-1629 (2001).

${ }^{49}$ T. Biewer, J. Sawyer, C. Smith, and C. Thomas, "Dual laser holography for in situ measurement of plasma facing component erosion (invited)," 89, Review of scientific instruments, 10J123 (2018).

${ }^{50}$ G. Litfin, C. R. Pollock, R. F. Curl, and F. K. Tittel, "Sensitivity enhancement of laser absorption spectroscopy by magnetic rotation effect," The Journal of Chemical Physics $\mathbf{7 2}$, 6602-6605 (1980). 
${ }^{51}$ R. A. Stern and J. A. Johnson, "Plasma ion diagnostics using resonant fluorescence," Phys. Rev. Lett. 34, 1548-1551 (1975).

${ }^{52}$ W. Kaiser and C. Garret, "Two-photon excitation in $\mathrm{CaF}_{2}: \mathrm{Eu}^{2+}$," Physical Review Letters 7, 229-231 (1961).

${ }^{53}$ D. S. Thompson, T. E. Steinberger, A. M. Keesee, and E. E. Scime, "Laser induced fluorescence of ar-i metastables in the presence of a magnetic field," 27, Plasma Sources Sci. Technol, 065007 (2018).

${ }^{54}$ A. Jain, Y. Wang, and W. D. Kulatilaka, "Three-photon-excited laser-induced fluorescence detection of atomic hydrogen in flames," Opt. Lett. 44, 5945-5948 (2019).

${ }^{55}$ W. Demtroeder, "Laser spectroscopy 1," in, 5th ed. (Springer, 2014).

${ }^{56}$ J. Amorim, G. Baravian, and J. Jolly, "Laser induced resonance fluorescence as a diagnostic technique in non-thermal equilibrium plasmas," Journal of Physics D: Applied Physics 33, R51-R65 (2000).

${ }^{57}$ W. C. Martin and C. H. Corliss, "The spectrum of singly ionized atomic iodine (i ii)," Journal of Research of the National Bureau of Standards-A. Physics and Chemistry 64A, 443-479 (1960).

${ }^{58}$ M. Galante, R. Magee, and E. Scime, "Two-photon laser induced fluorescence measurements of neutral density in a helicon plasma," Physics of Plasmas 21, 055704 (2014).

${ }^{59}$ E. Es-sebbar, Y. Benilan, A. Jolly, and M.-C. Gazzeau, "Characterization of an n 2 flowing microwave post-discharge by oes spectroscopy and determination of absolute ground-state nitrogen atom densities by talif," Journal of physics. D, applied physics 42, 135206 (2009).

${ }^{60}$ E. Es-Sebbar, C. Sarra-Bournet, N. Naudé, and N. Gherardi, "Absolute nitrogen atom density measurements by two-photon laser-induced fluorescence spectroscopy in atmospheric pressure dielectric barrier discharges of pure nitrogen," Journal of Applied Physics 106, 073302 (2009).

${ }^{61}$ D. Elliot, E. Scime, and Z. Short, "Novel calibration scheme for two-photon absorption laser induced fluorescence of hydrogen," Review of Scientific Instruments 87, 11E504 (2016). 
${ }^{62}$ S. Mazouffre, C. Foissac, P. Supiot, P. Vankan, R. Engeln, D. Schram, and N. Sadeghi, "Density and temperature in $\mathrm{n}$ atoms in the afterglow of a microwave discharge measured by two-photon laser-induced fluorescence technique," Plasma Sources Science and Technology 10, 168-175 (2001).

${ }^{63}$ R. Loudon, "The quantum theory of light," in, 2nd ed. (Clarendon Press, New York, 1983).

${ }^{64}$ M. Göppert-Mayer, "Über elementarakte mit zwei quantensprüngen," Annalen der Physik 401, Ph.D. Thesis, 273-294 (1931).

${ }^{65}$ K.-S. Chung, "Mach probes," Plasma Sources Science and Technology 21, 063001 (2012).

${ }^{66}$ N. Kafle, L. W. Owen, J. F. Caneses, T. M. Biewer, J. B. O. Caughman, D. C. Donovan, R. H. Goulding, and J. Rapp, "Plasma flow measurements in the prototype-material plasma exposure experiment (proto-mpex) and comparison with b2.5-eirene modeling," Physics of Plasmas 25, 052508 (2018).

${ }^{67}$ G. Sultan, G. Baravian, and J. Jolly, "Resonant three-photon ionization and two-photon laser-induced fluorescence of atomic oxygen in a discharge and post-discharge," Chemical Physics Letters 175, 37-42 (1990).

${ }^{68}$ I. Campbell and B. Arthur, "The association of oxygen atoms and their combination with nitrogen atoms," Proceedings of the Royal Society A 296, 222-232 (1967).

${ }^{69}$ H. Döbele, T. Mosbach, K. Niemi, and V. Schulz-von der Gathen, "Laser-induced fluorescence measurements of absolute atomic densities: concepts and limitations," Plasma Sources Science and Technology 14, S31-S41 (2005).

${ }^{70}$ K. Niemi, V. Schulz-von der Gathen, and H. Dbele, "Absolute calibration of atomic density measurements by laser-induced fluorescence spectroscopy with two-photon excitation," Journal of Physics D: Applied Physics 34, 2330-2335 (2001).

${ }^{71}$ S. Lasertechnik, Cobra stretch manual, 3rd ed., Sirah Lasertechnik GmbH (Heinrich-HertzStrae 11, 41516 Grevenbroich, Germany, 2011), Laser Description-4.

${ }^{72}$ H. Kuhn, "Atomic spectra," in, 2nd ed. (Academic Press, New York, 1962) Chap. 6. Hyperfine Structure and Isotope Shift, pp. 329-353.

${ }^{73} \mathrm{~S}$. Abrarov and B. Quine, The voigt/complex error function (second version), MATLAB Central File Exchange, Retrived September 3, 2020, 2020. 
${ }^{74}$ R. Janev, W. Langer, K. Evans Jr, and D. Post Jr, "Elementary processes in hydrogen-helium plasmas," in, 1st ed. (Springer, 1987), p. 44.

${ }^{75} \mathrm{P}$. Zeeman, Over den invloed eener magnetisatie op den aard van het door een stof uitgezonden licht, 1st ed. (Koninklijke Akademie van Wetenschappen te Amsterdam, 1896).

${ }^{76}$ K. S. Krane, "Introductory nuclear physics," in, 1st ed. (John Wiley and Sons, New York, 1988) Chap. 16. Nuclear Spin and Moments, pp. 610-619.

${ }^{77}$ F. Paschen and E. Back, "Liniengruppen magnetisch vervollstndigt," in, 1st ed. (Physica, 1926), pp. 261-273.

${ }^{78}$ H. E. White, "Introduction to atomic spectra," in, 1st ed. (McGraw-Hill Inc., New York, 1934) Chap. 18. Hyperfine Structure, pp. 352-385.

${ }^{79}$ D. Griffiths, "Introduction to quantum mechanics," in, 1st ed. (Prentice Hall, New Jersey, 1995), pp. 235-252.

${ }^{80} \mathrm{~K}$. Bonin and T. McIlrath, "Two-photon electric-dipole selection rules," Journal of the Optical Society of America B 1, 52-55 (1984).

${ }^{81} \mathrm{R}$. Boivin, Internal report pl-039: study of the different line broadening mechanisms for the laser induced fluorescence diagnostic of the helix and leia plasmas, tech. rep. (West Virginia University, 1998).

${ }^{82} \mathrm{R}$. Boivin, Internal report pl-050: zeeman splitting for lif transitions and de-convolution technique to extract ion temperature, tech. rep. (West Virginia University, 2001).

${ }^{83}$ R. Wynands, A. Nagel, S. Brandt, D. Meschede, and A. Weis, "Selection rules and line strengths of zeeman-split dark resonances," Phys. Rev. A 58, 196-203 (1997).

${ }^{84}$ R. D. Cowan, "The theory of atomic structure and spectra," in, 1st ed. (University of California Press, Berkeley, 1981) Chap. 17. External Fields and Nuclear Effects.

${ }^{85} \mathrm{M}$. Goeckner and J. Goree, "Laser induced fluorescence measurement of plasma ion temperatures: corrections for power saturation," Journal of Vacuum Science \& Technology A: Vacuum, Surfaces, and Films 7, 977-981 (1989).

${ }^{86}$ G. Severn, D. Edrich, and R. McWilliams, "Argon ion laser induced fluorescence with diode lasers," Review of Scientific Instruments 69, 10-15 (1998). 
${ }^{87}$ T. Kajiwara, K. Takeda, H. Kim, W. Park, T. Okada, M. Maeda, K. Muraoka, and M. Akazaki, "Application of laser fluorescence spectroscopy by two-photon excitation into atomic hydrogen density measurement in reactive plasmas," Japanese Journal of Applied Physics 29, L154-L156 (1990).

${ }^{88}$ S. Lasertechnik, Matisse user's guide, 2.2, Sirah Lasertechnik GmbH (Heinrich-Hertz-Strae 11, 41516 Grevenbroich, Germany, 2013), p. 16.

${ }^{89}$ Sirah Lasertechnik, Matisse $2 d r$, http : / / www . sirah.com/laser / cw-ring-lasers / matisse-dr, Retrieved May 19, 2020, 2020.

${ }^{90} \mathrm{~B}$. Instruments, Model 621 laser wavelength meter user guide, 4th ed., Bristol Instruments (770 Canning Parkway Victor, New York 14564, 2009).

${ }^{91}$ D. S. Thompson, M. F. Henriquez, E. E. Scime, and T. N. Good, "Confocal laser induced fluorescence with comparable spatial localization to the conventional method," Review of Scientific Instruments 88, 103506 (2017).

${ }^{92}$ E. Aguirre, "Spontaneous formation of ion holes and ion beams in expanding plasmas," $\mathrm{PhD}$ thesis (West Virginia University, West Virginia University Libraries, 1999), p. 47.

${ }^{93}$ M. Paul, S. Chakraborty Thakur, E. Scime, and G. R. Tynan, "Ion temperature measurements in pisces-rf: a liquid-cooled high-power steady-state helicon plasma device," Physics of Plasmas, Submitted (2020).

${ }^{94}$ T. E. Steinberger, M. T. Dufor, D. S. Thompson, and E. E. Scime, "Zeeman splitting measurements of magnetic fields in iodine plasma," Review of Scientific Instruments 89, 10C113 (2018).

${ }^{95}$ A. Kono and S. Hattori, "Radiative-lifetime measurements for i i and i ii," Journal of Optical Society of America 69, 253-255 (1978).

${ }^{96}$ D. Elliot, "Two-photon absorption laser induced fluorescence measurements for fusion class plasmas," PhD thesis (West Virginia University, West Virginia University Libraries, 2016).

${ }^{97}$ R. Boyd, "Nonlinear optics," in, 3rd ed. (Academic Press, Amsterdam, 2008), pp. 69-134.

${ }^{98}$ W. Koechner, "Solid-state laser engineering," in, 6th ed. (Springer-Verlag, New York, 2006), pp. 592-633. 
${ }^{99}$ G. Arfken, H. Weber, and F. Harris, "Mathematical methods for physicist: a comprehensive guide," in, 7th ed. (Academic Press, 2013), pp. 985-986.

${ }^{100}$ G. M. Stéphan, T. T. Tam, S. Blin, P. Besnard, and M. Têtu, "Laser line shape and spectral density of frequency noise," Phys. Rev. A 70, 043809 (2005).

${ }^{101}$ R. Pitts, S. Carpentier, F. Escourbiac, T. Hirai, V. Komarov, A. Kukushkin, S. Lisgo, A. Loarte, M. Merola, R. Mitteau, A. Raffray, M. Shimada, and P. Stangeby, "Physics basis and design of the iter plasma-facing components," Journal of Nuclear Materials 415, Proceedings of the 19th International Conference on Plasma-Surface Interactions in Controlled Fusion, S957-S964 (2011).

${ }^{102}$ J. Rapp, T. Biewer, J. Canik, J. Caughman, R. Goulding, D. Hillis, J. Lore, and L. Owen, "The development of plasma-material interaction facilities for the future of fusion technology," Fusion Science and Technology 64, 237-244 (2013).

${ }^{103}$ J. Rapp, A. Lumsdaine, C. Beers, T. Biewer T.M.and Bigelow, J. Caneses, J. Caughman, R. Goulding, N. Kafle, C. Lau, E. Lindquist, P. Piotrowicz, H. Ray, M. Showers, and the MPEX Team, "Latest results from proto-mpex and the future plans for mpex," Fusion Science and Technology 75, 654-663 (2019).

${ }^{104}$ N. Ohno, "Plasma detachment in linear devices," Plasma Physics and Controlled Fusion 59, 034007 (2017).

${ }^{105}$ J. Rapp, T. Biewer, T. Bigelow, J. Caughman, R. Duckworth, R. Ellis, D. Giuliano, R. Goulding, D. Hillis, R. Howard, T. Lessard, J. Lore, A. Lumsdaine, E. Martin, W. McGinnis, S. Meitner, L. Owen, H. Ray, G. Shaw, and V. Varma, "The development of the material plasma exposure experiment," IEEE Transactions on Plasma Science 44, 3456-3464 (2016). ${ }^{106}$ J. Rapp, T. Biewer, T. Bigelow, J. Caneses, J. Caughman, S. Diem, R. Goulding, R. Isler, A. Lumsdaine, C. Beers, T. Bjorholm, C. Bradley, J. Canik, D. Donovan, R. Duckworth, R. Ellis, V. Graves, D. Giuliano, D. Green, D. Hillis, R. Howard, N. Kafle, Y. Katoh, A. Lasa, T. Lessard, E. Martin, S. Meitner, G.-N. Luo, W. McGinnis, L. Owen, H. Ray, G. Shaw, M. Showers, and V. Varma, "Developing the science and technology for the material plasma exposure eXperiment," Nuclear Fusion 57, 116001 (2017).

${ }^{107}$ J. Rapp, "The challenges of plasma material interactions in nuclear fusion devices and potential solutions," Fusion Science and Technology 72, 211-221 (2017). 
${ }^{108}$ J. Rapp, T. M. Biewer, T. Bigelow, J. B. O. Caughman, R. Duckworth, D. Giuliano, R. H. Goulding, D. L. Hillis, R. Howard, R. J. Ellis, T. Lessard, J. D. Lore, A. Lumsdaine, E. Martin, W. D. McGinnis, S. J. Meitner, L. W. Owen, H. Ray, G. Shaw, and V. Varma, "The material plasma exposure experiment mpex: pre-design, development and testing of source concept," in 2015 ieee 26th symposium on fusion engineering (sofe) (2015), pp. 1-8.

${ }^{109}$ S. Hiroe, R. Colchin, G. Haste, F. Baity, D. Bates, L. Berry, T. Bigelow, R. Burris, J. Cobble, W. Davis, R. Donaldson, J. Glowienka, D. Hillis, H. Kimrey, R. Livesey, J. Mankin, M. McGuffin, D. Overbey, B. Peterson, D. Rasmussen, R. Richards, C. Schaich, G. Sullivan, D. Swain, T. Uckan, T. White, J. Wilgen, R. Wintenberg, K. Young, K. Connor, J. Goyer, R. Hickok, L. Solensten, F. Bieniosek, R. Juhala, T. Owens, D. Boyd, R. Mahon, V. Yun, B. Quon, Jaycor, O. Hankins, W. Casson, and K. Carpenter, "Summary of ELMO bumpy torus (EBT-s) experiments from 1982 to 1984," Nuclear Fusion 28, 2249-2263 (1988).

${ }^{110}$ T. M. Biewer, T. S. Bigelow, J. F. Caneses, S. J. Diem, D. L. Green, N. Kafle, and J. Rapp, "Observations of electron heating during $28 \mathrm{GHz}$ microwave power application in protompex," Physics of Plasmas 25, 024501 (2018).

${ }^{111}$ L. C. Ingesson, B. Alper, B. J. Peterson, and J.-C. Vallet, "Chapter 7: tomography diagnostics: bolometry and soft-x-ray detection," Fusion Science and Technology 53, 528-576 (2008).

${ }^{112}$ M. Bernert, T. Eich, A. Burckhart, J. C. Fuchs, L. Giannone, A. Kallenbach, R. M. McDermott, and B. Sieglin, "Application of axuv diode detectors at asdex upgrade," Review of Scientific Instruments 85, 033503 (2014).

${ }^{113}$ P. Piotrowicz, "Physics of the helicon antenna on the prototype materials exposure experiment," MA thesis (Univeristy of Illinois-Urbana-Champaign, 2018), pp. 26-28.

${ }^{114}$ M. Light, I. D. Sudit, F. F. Chen, and D. Arnush, "Axial propagation of helicon waves," Physics of Plasmas 2, 4094-4103 (1995).

${ }^{115} \mathrm{MKS}$ Instruments, Baratron capacitance manometers, https : //www. mksinst.com/n/ baratron-capacitance-manometers, Retrieved May 18, 2020, May 2020.

${ }^{116}$ N. Kafle, "A transport study of high density helicon plasma discharges in the prototype material plasma exposure experiment," PhD thesis (University of Tennessee, 2019). 
${ }^{117}$ Luxtron Corporation, Fluoroptic corporation, https : / / www . lambdaphoto . co . uk/pdfs / fot1_labkit.pdf, Retrieved May 18, 2020, 2005.

${ }^{118}$ H. B. Ray, T. M. Biewer, D. T. Fehling, R. C. Isler, and E. A. Unterberg, "Spectral survey of helium lines in a linear plasma device for use in helios imaging," Review of Scientific Instruments 87, 11E711 (2016).

${ }^{119}$ M. Showers, T. M. Biewer, J. B. O. Caughman, D. C. Donovan, R. H. Goulding, and J. Rapp, "Heat flux estimates of power balance on proto-mpex with ir imaging," Review of Scientific Instruments 87, 11D412 (2016).

${ }^{120}$ M. L. Reinke and I. H. Hutchinson, "Two dimensional radiated power diagnostics on alcator c-mod," Review of Scientific Instruments 79, 10F306 (2008).

${ }^{121}$ A. Huber, K. McCormick, P. Andrew, P. Beaumont, S. Dalley, J. Fink, J. Fuchs, K. Fullard, W. Fundamenski, L. Ingesson, F. Mast, S. Jachmich, G. Matthews, P. Mertens, V. Philipps, R. Pitts, S. Sanders, and W. Zeidner, "Upgraded bolometer system on jet for improved radiation measurements," Fusion Engineering and Design 80, 1327 -1334 (2007).

${ }^{122}$ G. A. Wurden and B. J. Peterson, "Imaging bolometry development for large fusion devices," Conference workshop; Diagnostics for Experimental Thermonuclear Fusion Reactors 2 (1998).

${ }^{123}$ G. van Eden, M. Reinke, S. Brons, G. van der Bijl, B. Krijger, R. Lavrijsen, S. Huber, R. Perillo, M. van de Sanden, and T. Morgan, "Plasma radiation studies in magnum-PSI using resistive bolometry," Nuclear Fusion 58, 106006 (2018).

${ }^{124}$ E. Scime, R. Boivin, J. Kline, and M. Balkey, "Microwave interferometer for steady-state plasmas," Review of Scientific Instruments 72, 1672-1676 (2001).

${ }^{125}$ H. J. Hartfuss, T Geist, and M Hirsch, "Heterodyne methods in millimetre wave plasma diagnostics with applications to ECE, interferometry and reflectometry," Plasma Physics and Controlled Fusion 39, 1693-1769 (1997).

${ }^{126}$ C. Bishop, "Vacuum deposition onto webs, films, and foils," in, 2nd ed. (Elsevier, 2011), pp. 90-92.

${ }^{127}$ I. Hutchinson, "Principles of plasma diagnostics," in, 2nd ed. (Cambridge University Press, 2002), pp. 94-99. 
${ }^{128}$ T. M. Biewer, S. Meitner, J. Rapp, H. Ray, and G. Shaw, "First results from the thomson scattering diagnostic on proto-mpex," Review of Scientific Instruments 87, $11 \mathrm{E} 518$ (2016).

${ }^{129}$ R. C. MacCallum, M. W. Browne, and H. M. Sugawara, "Power analysis and determination of sample size for covariance structure modeling," Psychological Methods 1, 130-149 (1996).

${ }^{130}$ T. B. Smith, B. B. Ngom, J. A. Linnell, and A. D. Gallimore, "Diode laser induced fluorescence of xenon ion velocity distributions," in 41st aiaa/asme/sae/asee joint propulsion conference and exhibit (July 2005).

${ }^{131}$ W. Demtroeder, "Laser spectroscopy 2," in, 5th ed. (Springer, 2014).

${ }^{132}$ E. H. Martin, A. Zafar, J. B. O. Caughman, R. C. Isler, and G. L. Bell, "Applications of doppler-free saturation spectroscopy for edge physics studies (invited)," Review of Scientific Instruments 87, 11E402 (2016).

${ }^{133}$ R. F. Boivin, J. L. Kline, and E. E. Scime, "Electron temperature measurement by a helium line intensity ratio method in helicon plasmas," Physics of Plasmas 8, 5303-5314 (2001).

${ }^{134}$ Z. Wu, M. Kitano, W. Happer, M. Hou, and J. Daniels, "Optical determination of alkali metal vapor number density using faraday rotation," Appl. Opt. 25, 4483-4492 (1986).

${ }^{135}$ T. Killian, "Thermionic phenomena caused by vapors of rubidium and potassium," Phys. Rev. 27, 578-587 (1926).

${ }^{136}$ P. Y. Peterson, A. D. Gallimore, and J. M. Haas, "An experimental investigation of the internal magnetic field topography of an operating hall thruster," Physics of Plasmas 9, $4354-4362$ (2002).

${ }^{137}$ L. W. Owen, J. Rapp, J. Canik, and J. D. Lore, "Transport modeling of convection dominated helicon discharges in proto-mpex with the b2.5-eirene code," Physics of Plasmas 24, 112504 (2017).

${ }^{138}$ R. M. Magee, M. E. Galante, N. Gulbrandsen, D. W. McCarren, and E. E. Scime, "Direct measurements of the ionization profile in krypton helicon plasmas," Physics of Plasmas 19, 123506 (2012).

${ }^{139}$ D. Marinov, C. Drag, C. Blondel, O. Guaitella, J. Golda, B. Klarenaar, R. Engeln, V. Schulzvon der Gathen, and J Booth, "Pressure broadening of atomic oxygen two-photon absorption laser induced fluorescence," Plasma Sources Science and Technology 25, 06LT03 (2016). 
${ }^{140}$ S. Gerstenkorn, P. Luc, J. Verges, and J. Chevillard, "Atlas du spectre d'absorption de la molécule d'iode," in, 2nd ed. (Laboratoire Amié Cotton, Orsay, France, 1978), p. 44.

${ }^{141}$ M Showers, P. A. Piotrowicz, C. J. Beers, T. M. Biewer, J Caneses, J Canik, J. B. O. Caughman, D. C. Donovan, R. H. Goulding, A Lumsdaine, N Kafle, L. W. Owen, J Rapp, and H Ray, "Power accounting of plasma discharges in the linear device proto-MPEX," Plasma Physics and Controlled Fusion 60, 065001 (2018).

${ }^{142}$ R. Schneider, X. Bonnin, K. Borrass, D. P. Coster, H. Kastelewicz, D. Reiter, V. A. Rozhansky, and B. J. Braams, "Plasma edge physics with b2-eirene," Contributions to Plasma Physics 46, 3-191 (2006).

${ }^{143}$ F. Biraben, B. Cagnac, and G. Grynberg, "Experimental evidence of two-photon transition without doppler broadening," Physical Review Letters 32, 643-645 (1974).

${ }^{144} \mathrm{P}$. Keiter, "Experimental investigation of ion temperature anisotropy driven instabilities in a high beta plasma," PhD thesis (West Virginia University, West Virginia University Libraries, 1999), pp. 57-67.

${ }^{145}$ R. Jahn and E. Choueiri, "Electric propulsion," in (2003), pp. 125-141.

${ }^{146}$ J. A. Linnell and A. D. Gallimore, "Efficiency analysis of a hall thruster operating with krypton and xenon," Journal of Propulsion and Power 22, 1402-1412 (2006).

${ }^{147}$ M. R. Nakles, W. A. Hargus, J. J. Delgado, and R. L. Corey, "A performance comparison of xenon and krypton propellant on an spt-100 hall thruster," in 32nd international electric propulsion conference (September 2011).

${ }^{148}$ M. N. A. Beurskens, T. H. Osborne, P. A. Schneider, E. Wolfrum, L. Frassinetti, R. Groebner, P. Lomas, I. Nunes, S. Saarelma, R. Scannell, P. B. Snyder, D. Zarzoso, I. Balboa, B. Bray, M. Brix, J. Flanagan, C. Giroud, E. Giovannozzi, M. Kempenaars, A. Loarte, E. de la Luna, G. Maddison, C. F. Maggi, D. McDonald, R. Pasqualotto, G. Saibene, R. Sartori, E. R. Solano, M. Walsh, and L. Zabeo, "H-mode pedestal scaling in diii-d, asdex upgrade, and jet," Physics of Plasmas 18, 056120 (2011).

${ }^{149}$ A. Leonrad, "Edge-localized-modes in tokamaks," Physics of Plasmas 21, 090501 (2014).

${ }^{150}$ J. Rapp, C. Beers, T. Biewer, T. Bigelow, J. Caneses, J. Caughman, S. Diem, R. Goulding, R. Isler, N. Kafle, L. Owen, and M. Showers, "Operational domain of proto-mpex," Fusion Engineering and Design 136, $712-716$ (2018). 
${ }^{151}$ C. J. Beers, R. H. Goulding, R. C. Isler, E. H. Martin, T. M. Biewer, J. F. Caneses, J. B. O. Caughman, N. Kafle, and J. Rapp, "Helicon plasma ion temperature measurements and observed ion cyclotron heating in proto-mpex," Physics of Plasmas 25, 013526 (2018).

${ }^{152}$ M. Paul, T. Steinberger, E. Lister, S. Chakraborty Thakur, D. Artis, N. Arnold, T. Hall, S. LeBlanc, E. Thomas, E. Scime, and G. Tynan, "Compact, portable, laser induced fluorescence diagnostic for laboratory plasma sources," Review of Scientific Intruments, Submitted (2020). 